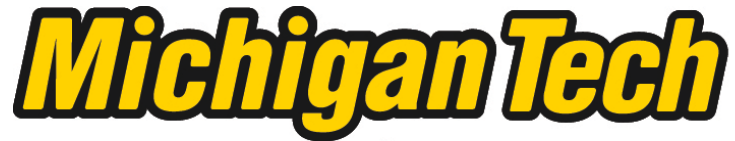 \\ Michigan Technological University Create the Future Digital Commons @ Michigan Tech
}

Dissertations, Master's Theses and Master's Reports - Open

Dissertations, Master's Theses and Master's

Reports

2010

Influence of traumatic impaction and pathological loading on knee menisci

Megan Leigh Killian

Michigan Technological University

Follow this and additional works at: https://digitalcommons.mtu.edu/etds

Part of the Biomedical Engineering and Bioengineering Commons

Copyright 2010 Megan Leigh Killian

\section{Recommended Citation}

Killian, Megan Leigh, "Influence of traumatic impaction and pathological loading on knee menisci", Dissertation, Michigan Technological University, 2010.

https://doi.org/10.37099/mtu.dc.etds/285

Follow this and additional works at: https://digitalcommons.mtu.edu/etds

Part of the Biomedical Engineering and Bioengineering Commons 


\title{
THE INFLUENCE OF TRAUMATIC IMPACTION AND PATHOLOGICAL LOADING ON KNEE MENISCI
}

\author{
By \\ Megan Leigh Killian \\ A DISSERTATION \\ Submitted in partial fulfillment of the requirements for the degree of \\ DOCTOR OF PHILOSOPHY \\ in Biomedical Engineering \\ MICHIGAN TECHNOLOGICAL UNIVERSITY
}

2010

C2010 Megan Leigh Killian 
This dissertation, "The Influence of Traumatic Impaction and Pathological Loading on Knee Menisci," is hereby approved in partial fulfillment of the requirements for the Degree of DOCTOR OF PHILOSOPHY IN BIOMEDICAL ENGINEERING.

Department of Biomedical Engineering

Dissertation Advisor:

\author{
Tammy Haut Donahue
}

Department Chair:

Sean Kirkpatrick

Date 


\section{Dedication}

This dissertation is dedicated to my late grandfather, Robert Lee Rauch, who passed away on Sunday, October $17^{\text {th }}, 2010$. He was undoubtedly the most enthusiastic person I have ever known, and was always driven by the accomplishments of others. He lived his life full of love for his family and his history, and I hope to live my life in a reflection of his, always asking the next question and driven to resolve the how's and the why's. 


\section{Table of Contents}

LIST OF FIGURES __ ix

LIST OF TABLES __ xii

PREFACE —

ACKNOWLEDGEMENTS _ _ Xiv

DEFINITIONS _

LIST OF ABBREVIATIONS __ xvii

ABSTRACT__ xviii

CHAPTER 1 - INTRODUCTION___ 1

Overview of the Knee

The Development of the Knee and Meniscus ___ 3

Composition \& Structure of the Meniscus __ 7

Mechanics of the Knee Meniscus __ 12

Meniscal Tears__ 13

Mechanotransduction of the Meniscus _ 15

Molecules Involved in Soft Tissue Degradation__ 19

Models for Investigating Knee OA __ 22

Problem Statement

Catabolic Behavior Following Mechanical Loading of the Meniscus ___ 26 Effect of Tibiofemoral Injury on the Meniscus __ 27 Research Aims__ 27

CHAPTER 2 - CATABOLIC RESPONSE OF MENISCAL TISSUE EXPLANTS TO DYNAMIC COMPRESSIVE STRAIN

Abstract__ 29

Introduction _ 29 
Materials and Methods _ 31

Mechanical Stimulation 31

Gene Expression 32

Western Blotting _ 34

Data Analysis — 35

Results _ 36

Discussion _ 36

Recommendations _ 40

CHAPTER 3 - IN VITRO INHIBITION OF COMPRESSION-INDUCED CATABOLIC GENE EXPRESSION IN MENISCAL EXPLANTS FOLLOWING TREATMENT WITH IL-1 RECEPTOR ANTAGONIST _ 42

Abstract 42

Cyclic loading protocol__ 45

RNA extraction 45

Reverse-transcription-polymerase chain reaction __ 46

Real-time quantitative-PCR

$\begin{array}{ll}\text { Statistical analysis } & 47\end{array}$

Results _ 48

Influence of IL-1RA treatment following dynamic compression of meniscal explants 48

Discussion _ 49

Conclusions

Recommendations _ 53

CHAPTER 4 - SPATIAL DISTRIBUTION OF SULFATED GAG IN HEALTHY LAPINE MENISCI

Abstract $\quad 57$

Introduction

Materials and Methods 59

Detection of Sulfated $\overline{G A G \ldots} 60$

Collagen Type I and II Distribution __ 60

Cell Density and Tissue Area Measurements ___ 61

Statistical Analysis __ 61 
Results

Discussion

CHAPTER 5 - TRAUMATIC ANTERIOR CRUCIATE LIGAMENT TEAR AND ITS IMPLICATIONS ON MENISCAL DEGRADATION

Abstract 74

Introduction 75

Materials and Methods 77

Animal Models

Morphological Analysis

Results

Gross Pathology and Grading of Meniscus 79

Histopathology

Discussion 81

CHAPTER 6 - REGIONAL CHANGES IN CELLULAR AND TISSUE MORPHOLOGY OF MENISCI FOLLOWING TRAUMATIC IMPACTION AND ACL TEAR 88

Abstract 88

Introduction 88

Materials and Methods 90

Histomorphometry 92

Statistics 95

Results 95

Discussion 98

Recommendations 103

Acknowledgements 103 


\section{CHAPTER 7 - ACUTE CELL VIABILITY OF THE MENISCUS AND CARTILAGE AND NO RELEASE OF JOINT TISSUES FOLLOWING TRAUMATIC IMPACTION}

Abstract 108

Introduction 108

Materials and Methods 109

Traumatic Impaction 109 Cell viability

Nitric oxide release 110

Statistics 111 111

Results 112

Discussion 113

Recommendations 116

CHAPTER 8 - SIGNIFICANCE OF RESEARCH 123

Understanding the Etiology of Osteoarthritis 123

Development of More Reliable Animal Models for Osteoarthritis 125

BIBLIOGRAPHY 127

APPENDIX A: DATA 162

APPENDIX B: PROTOCOLS 169

Decalcification and embedding: Histology prep protocol 170

Safranin O staining protocol Alizarin Red protocol Immunohistochemical staining for Col I/II- protocol $R T-P C R$ protocol Real Time PCR (QPCR) protocol 176

Western blotting protocol

Drop sled control protocol Rabbit handling and euthanasia protocol Acute response - Rabbit dissection protocol 177 178 180 184 189 194 195 Cell viability protocol 197 199 
APPENDIX C: COPYRIGHT 200

Copyright permission (Figure $1-1 \& 1-6)+201$

Copyright permission (Figure 1-2) __ 203

Copyright permission (Figure 1-3)_ 206

Copyright permission (Figure 1-5)_ 207

Copyright permission (Figure 1-8) 212

Copyright permission (Figure 1-8) 216

Copyright permission (Figure 1-8)_ 221

Copyright permission (Figure 1-8) 225

Copyright permission (Chapter 2) 229

Copyright permission (Chapter 4) _ 234

Copyright permission (Chapter 5) _ 237

$I A C U C \_242$ 


\section{List of Figures}

Figure 1-1. Anatomy of the knee and the progression of osteoarthritis. 2

Figure 1-2. Histology of the meniscus in human fetuses. 4

Figure 1-3. Photomicrographs of developing menisci. 6

Figure 1-4. Schematic of medial and lateral menisci, with coronal cross-section. 7

Figure 1-5. Schematic representation of the domain organisation (a) and structure (b) of human cartilage aggrecan. 9

Figure 1-6. Potential tear types and red/white zones of the menisci. 14

Figure 1-7. Catabolic biochemical pathway induced by compressive loading. 20

Figure 1-8. Timeline of degradation following ACLT. 25

Figure 2-1. Gene expression of ADAMTS-4 and -5 following $0 \%, 10 \%$ or $20 \%$ dynamic compressive strain.

Figure 2-2. Immunoblot of endogenously produced protein in superficial (S) and deep (D) explants compressed to 0,10 , or $20 \%$ strain levels. 41

Figure 3-1. Relative expression of catabolic genes for both superficial and deep explants with and without IL-1RA treatment. 53

Figure 3-2. Relative expression of COX-2and iNOS, genes for both superficial and deep explants, as well as aggrecan gene for superficial explants only, with and without IL-1RA treatment. 55

Figure 3-3. Schematic of mechanically-induced IL-1 pathway and IL-1RA blockade. 56 Figure 4-1. Sectioning of meniscal regions. 68

Figure 4-2. (A) Regional comparisons in percentage of GAG coverage between medial and lateral menisci and (B) representative SafO histological images. 69

Figure 4-3. (A) Zonal comparisons of percentage of GAG coverage in medial and lateral menisci and $(\mathrm{B})$ representative SafO histological images. 70

Figure 4-4. Collagen type I immuno-fluorescence staining of medial and lateral meniscal sections for anterior, central, and posterior regions. 71

Figure 4-5. Collagen type II immuno-fluorescence staining of medial and lateral meniscal sections for anterior, central, and posterior regions. 71 
Figure 4-6. Regional comparisons in tissue area $\left(\mathrm{mm}^{2}\right)$ between medial and lateral menisci.

Figure 4-7. Zonal comparisons in tissue area $\left(\mathrm{mm}^{2}\right)$ between regions of the medial and lateral menisci. 72

Figure 4-8. Regional comparisons in cell density $\left(\right.$ cells $/ \mathrm{mm}^{2}$ ) between medial and lateral menisci. 73

Figure 4-9. Zonal comparisons in cell density $\left(\mathrm{cells} / \mathrm{mm}^{2}\right)$ between regions of the medial and lateral menisci. 73

Figure 5-1. Schematic of traumatic impaction. 86

Figure 5-2. Gross morphological assessment of lateral and medial menisci of uninjured, acute TEAR, 12 wk TEAR, and 12 wk ACLT animals. 86

Figure 5-3. Microscopic structural and cellular findings of control (A-D), 12 wk TEAR (E-H), and 12 wk ACLT (I-L) left menisci. 87

Figure 6-1. GAG coverage as a percentage of cross-sectional area and micromorphology of control, TEAR, ACLT, and sham ACLT menisci. 104

Figure 6-2. Menisci stained with alizarin red of control (A), TEAR (B \& D), and ACLT (C \& E) groups. 105

Figure 6-3. Cell morphology of healthy (A), TEAR (B), and ACLT (C) from the anterior region of the lateral menisci..

Figure 6-4. Tissue area $\left(\mathrm{mm}^{2}\right)$ and cell density $\left(\right.$ cells $\left./ \mathrm{mm}^{2}\right)[\mathrm{mean}+\mathrm{SE}]$ for for control, TEAR, ACLT, and sham ACLT medial (M) and lateral (L) anterior (A), central $(\mathrm{C})$, and posterior $(\mathrm{P})$ regions. 107

Figure 7-1. Impact experiments were performed by dropping a gravity-accelerated mass onto the flexed tibial-femoral joint.

Figure 7-2. Gross morphology of menisci immediately after dissection in control, unimpacted right knee (A) and impacted left knee resulting in ACL tear (B). 118

Figure 7-3. Cell viability of menisci from control and impacted knees. 119

Figure 7-4. Cell viability of cartilage, lateral menisci, and medial menisci normalized to control limb. 
Figure 7-5. Zonal comparison of cell viability of articular cartilage for control and impacted limbs.

Figure 7-6. Cell viability of control (left column) and impacted (right column) tibiofemoral cartilage. 121

Figure 7-7. NO release of meniscotibial (T) and femoral (F) tissue from control and impacted limbs measured in the culture media after 12 and 24 hours of incubation. 122 


\section{List of Tables}

Table 1-1. Proteoglycans and glycosaminoglycans of the meniscus.

Table 2-1. Primer sequences used for specific genes.

Table 3-1. Primer sequences used for specific genes. 48

Table 6-1. Scoring system for assessment of histomorphometric changes in menisci, classifying synovial layer, cell morphology, and quality of GAG staining of meniscal sections.

Table 6-2. Grading and characterization of microscopic meniscal damage. 94

Table 6-3. Alizarin red scoring criteria for menisci from healthy and injured limbs. 94

Table 6-4. Morphometric scoring of both medial (M) and lateral (L) meniscal sections for Control, ACLT, TEAR and ACLT sham groups.

Table 6-5. Grade of alizarin red staining. 96

Table 7-1. Morphological assessment including meniscal and ACL tears of animals. 112 


\section{Preface}

The work presented in this dissertation is a compilation of my own review of the literature as well as my own work published in several peer-reviewed manuscripts. However, it is important to point out that I am not the sole contributor to the studies of pathological loading and traumatic impaction on knee menisci. However, the data presented in this dissertation and the conclusions derived are my own work. In Chapters 2 and 3, I did not perform the dynamic compression exercises on meniscal explants; however, I was responsible for gene and protein assays, interpreting the results, and disseminating the findings. In chapters 4, 5, and 6, the experimental procedures of traumatic knee impaction were performed by collaborators at Michigan State. However, all analyses performed subsequent to harvesting of the menisci, as well as the dissemination of the findings, were performed by me. 


\section{Acknowledgements}

Though the text in this dissertation was written by me, the words on these pages would never have made it without the help of several people. Of the many people I have to thank, it is my advisor, Dr Tammy Haut Donahue, to whom I owe the greatest appreciation. She has demonstrated to me the importance of balancing work and life, and has encouraged my ventures outside of academics. She has provided me so many opportunities to explore my own research ideas independently, and she has done nothing but encourage me to design, execute, and complete several projects with the freedom to make mistakes. Throughout my education thus far, from undergrad to my dissertation defense, she has provided me with outstanding mentorship and irreplaceable friendship. Her enthusiastic approach to education and innovation in research has been a hallmark that I hope to reflect in my future career in academia.

My sanity was maintained throughout my doctoral work with the help of my lab-mates. In particular, I want to thank Adam Abraham for great discussions, union breaks brainstorming, insight into astronomy, and for being there when I needed a laugh. I feel fortunate to have worked with Nicole Lepinski and John Moyer in the Soft Tissue Mechanics Lab, and their intellect, attitude, and contributions went above and beyond what they learned in the classroom. I also want to thank Diego Villegas, Matt Nelsen, and Karen Hauch, for helping to get me settled into the lab during the first year of grad school when my advisor was on sabbatical.

I am thankful for the generous insight of my home department. Dr Seth Donahue provided my first exposure to the field of biomechanics as I sat in his undergraduate biomechanics course, and I will always appreciate his teaching style, humor, and approachable attitude. Dr Michael Neuman has been an integral part of my education

since my time as an undergraduate at Michigan Tech, and I'll always remember first meeting him not in the biomed office, but at the Fitz. I would like to thank my committee, Dr Rupak Rajachar, Dr Jeremy Goldman, and Dr Gregory Odegard, for encouraging me to think outside the box. With help from them as members of my dissertation committee, 
I have developed potential research ideas for a future in academics. I am also grateful for the help of Judy Schaefer and JoAnne Stimac, who have provided me with everything necessary to get through many of the hoops throughout the graduate process.

Additionally, working with external collaborators, including Dr Roger Haut and the Orthopaedic Biomechanics group at Michigan State University, has been an absolute delight. The work with Michigan State catapulted my dissertation research in a new direction, but ultimately provided me with the opportunity to help design and develop integral orthopaedic research that will carry on for years to come.

Most importantly, none of this would have been possible without the love and support of my family. My parents, who have supported academic pursuits, my grandmother and late grandfather (to whom this dissertation is dedicated to), my sister, my cousin, Amy, and my boyfriend, Adam, have all helped me with emotional support throughout this process. The friends I've made during my time at Michigan Tech have been my surrogate family, as well, and I am thankful for having met such extraordinary people in such a small town. Particularly, I'd like to thank my training sidekicks, my local bike shop, and my girls on Team Mega Tough.

Finally, I am thankful of the financial support from the Marshall Family Fellowship, the DeVlieg Fellowship, the Michigan Tech Graduate Finishing Fellowship, the Michigan Space Grant Consortium Graduate Fellowship (2 years of support), the BRC Finishing Fellowship, the BRC Travel Grant, as well as the CDC which funded parts of the research discussed in this dissertation. 


\section{Definitions}

Osteoarthritis

Meniscus

in vitro

in vivo

mechanotransduction Mechanism by which cells turn mechanical stimuli into a chemical

Whole organ joint disease of cartilage deterioration, joint space narrowing, pain and disability where the only viable therapeutic option may be joint replacement

C-shaped fibrocartilagenous tissue in the knee that attaches at two distinct anterior and posterior attachments into the tibial plateau of humans

Procedure performed in a controlled laboratory environment Experimentation using whole, living organism

response 


\section{List of Abbreviations}

ACL- Anterior cruciate ligament

ACLT- Animal group subjected to surgical transection of the ACL

OA- Osteoarthritis

TEAR- $\quad$ Animal group subjected to traumatic impaction without constraint of the tibia, resulting in ACL rupture

SafO- Fast-Green Safranin-O staining use for the detection and spatial distribution of sulfated glycosaminoglycans 


\section{Abstract}

Nearly half of the US population faces the risk of developing knee osteoarthritis (OA). Both in vitro and in vivo studies can aid in a better understanding of the etiology, progression, and advancement of this debilitating disorder. The knee menisci are fibrocartilagenous structures that aid in the distribution of load, attenuation of shock, alignment and lubrication of the knee. Little is known about the biochemical and morphological changes associated with knee menisci following altered loading and traumatic impaction, and investigations are needed to further elucidate how degradation of this soft tissue advances over time. The biochemical response of porcine meniscal explants was investigated following a single bout of dynamic compression with and without the treatment of the pharmaceutical drug, anakinra (IL-1RA). Dynamic loading led to a strain-dependent response in both anabolic and catabolic gene expression of meniscal explants. By inhibiting the Interleukin-1 pathway with IL-1RA, a marked decrease in several catabolic molecules was found. From these studies, future developments in OA treatments may be developed. The implementation of an in vivo animal model contributes to the understanding of how the knee joint behaves as a whole. A novel closed-joint in vivo model that induces anterior cruciate ligament (ACL) rupture has been developed to better understand how traumatic injury leads to OA. The menisci of knees from three different groups (healthy, ACL transected, and traumatically impacted) were characterized using histomorphometry. The acute and chronic changes within the knee following traumatic impaction were investigated. The works presented in this dissertation have focused on the characterization, implementation, and development of mechanically-induced changes to the knee menisci. 


\section{Chapter 1 - INTRODUCTION}

Nearly half of the US population faces the risk of developing knee osteoarthritis (OA), which causes disability, pain and hospitalization. For those over the age of 65, joint disease accounts for half of all disabilities. As it is projected that elderly will account for $25 \%$ of the population by the year $2020^{1}$, the importance of understanding the etiology of $\mathrm{OA}$ is evident in order to prevent future disease risks. A plethora of variables are thought to influence the onset and progression of knee OA, including obesity, gender, age, and genetics, as well as traumatic injury to the tibiofemoral joint ${ }^{1,2}$. The Center for Disease Control and Prevention recently reported obesity as the strongest known contributor to the risk of knee OA, due to extra weight overloading the joint. Pathological OA is defined as severe, localized cartilage damage and fibrillation and characterized by an imbalance in catabolic and anabolic activity of chondrocytes ${ }^{3}$. Articular cartilage is avascular, aneural, alymphatic, and moderately cellularized, resulting in slow matrix turnover and reduced affinity for repair following damage ${ }^{3}$.

Although the mechanisms of $\mathrm{OA}$ are somewhat unclear, long-term investigations of joint damage and subsequent deterioration have encouraged scientists and clinicians to investigate the role of the menisci in the etiology of the disease ${ }^{4-7}$ (Figure 1-1). The menisci are multifunctional tissues in the knee that aid in tibiofemoral alignment, lubrication, load distribution, shock absorption, and protect the underlying cartilage. It is well documented that either complete or partial removal of the menisci will result in $\mathrm{OA}^{2}$, 8,9 , yet the mechanisms responsible for meniscal degeneration are currently unknown. Both partial meniscectomy and anterior cruciate ligament (ACL) transection lead to excessive mechanical loading and disruption of structural proteins within the meniscus ${ }^{10}$. It has been observed that joint disruption leads to the production of matrix degrading enzymes, including nitric oxide (NO) and metalloproteinases ${ }^{11-13}$. Several studies have demonstrated a reduction in joint space due to meniscal degradation prior to any cartilage damage ${ }^{8,14-16}$. Therefore, it is imperative to investigate the mechanism of meniscal 
degeneration resulting from altered loading and a means by which this degeneration can be slowed or prevented.

\section{Overview of the Knee}

The knee is a complex, condyloid, synovial joint located in the lower extremity, and is responsible for weight-bearing and mobility activities. The knee provides shock

attenuation from impact loading during walking, running, and jumping. The patellofemoral joint is comprised of a sesamoid bone (the patella) which articulates in the femoral groove. The patella acts to protect the deeper synovial joint from impact. The tibiofemoral joint is a complex hinge joint that is encapsulated by synovial tissue, aiding in joint lubrication. The tibiofemoral joint incorporates many soft tissue structures to maintain knee stability. The anterior and posterior cruciate ligaments, as well as the biarticulate nature of the hamstrings and quadriceps muscle groups, contribute to the prevention of frontal plane translation (subluxation) between the tibia and femur. Sagittal translation of the knee is inhibited by medial and lateral collateral ligaments. The fibrocartilagenous structures atop the tibial plateaus, known as the menisci, contribute to dynamic knee stability. Although the primary motion of the knee is flexion and

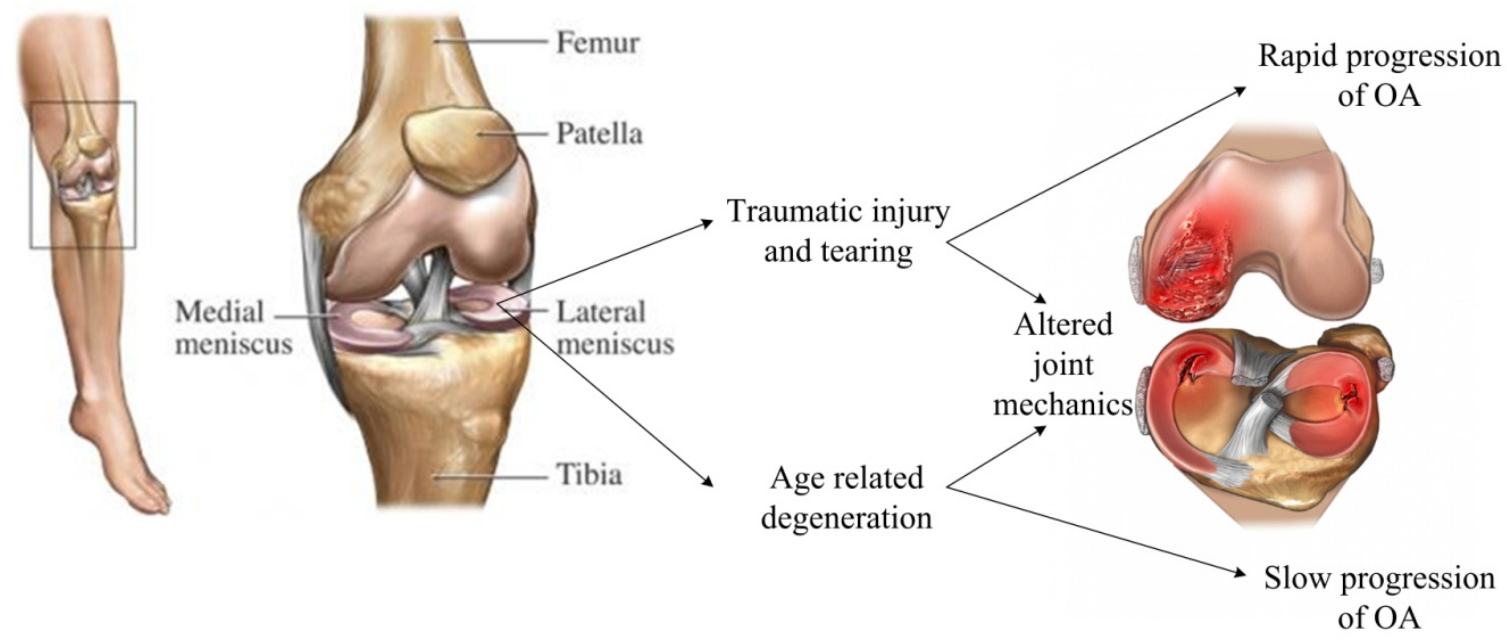

Figure 1-1. Anatomy of the knee and the progression of osteoarthritis. Illustrations provided by Nucleus Medical Media, copyright 2010. All rights reserved, used with permission. 
extension, some interplay in pronation and supination, as well as internal and external rotation, occurs. Excessive sagittal or transverse planar motion, however, can result in ligament or meniscal tear, bone bruising, muscle injury, or other problems ${ }^{17-23}$.

Many factors can contribute to malalignment of the knee. For example, cruciate ligament tears demonstrate increased joint laxity between the tibia and femur, resulting in diminished proprioception, delayed reflex responses to anterior tibial translation, and changes in neuromuscular control strategies during dynamic maneuvers ${ }^{24-26}$. This joint laxity also contributes to altered loading patterns on the meniscus ${ }^{27}$. The progression of osteoarthritis (OA), also known as degenerative joint disease, is likely influenced by the altered kinematics of the knee joint. The maintenance of healthy stabilizing structures of the knee has been heavily emphasized in the literature $2,3,13,27-38$.

\section{The Development of the Knee and Meniscus}

The development of the knee has been investigated for over half a century ${ }^{39-42}$. Normal joint formation consists of two distinct phases: blastema differentiation and joint cavitation. The human fetus begins to develop limb buds around 3 weeks after fertilization, and differentiation of the femur, tibia, and fibula ensues around 6 weeks ${ }^{39}$. Upon cavitation, two distinct articulating surfaces are formed ${ }^{43}$, and femoral condyles begin to take shape 7weeks after fertilization. Within the next week of development the lateral menisci begins to appear and demonstrate high cellularity ${ }^{39}$. By 14 weeks, the anterior horn of the lateral meniscus demonstrates parallel-aligned spindle cells whereas the cells of the medial meniscal anterior horns are randomly arranged (Figure $1-2 ;^{40}$ ). Organizational changes in the menisci occur during the next few weeks of development (Figure 1-2; ${ }^{40}$ ). 
Lateral meniscus
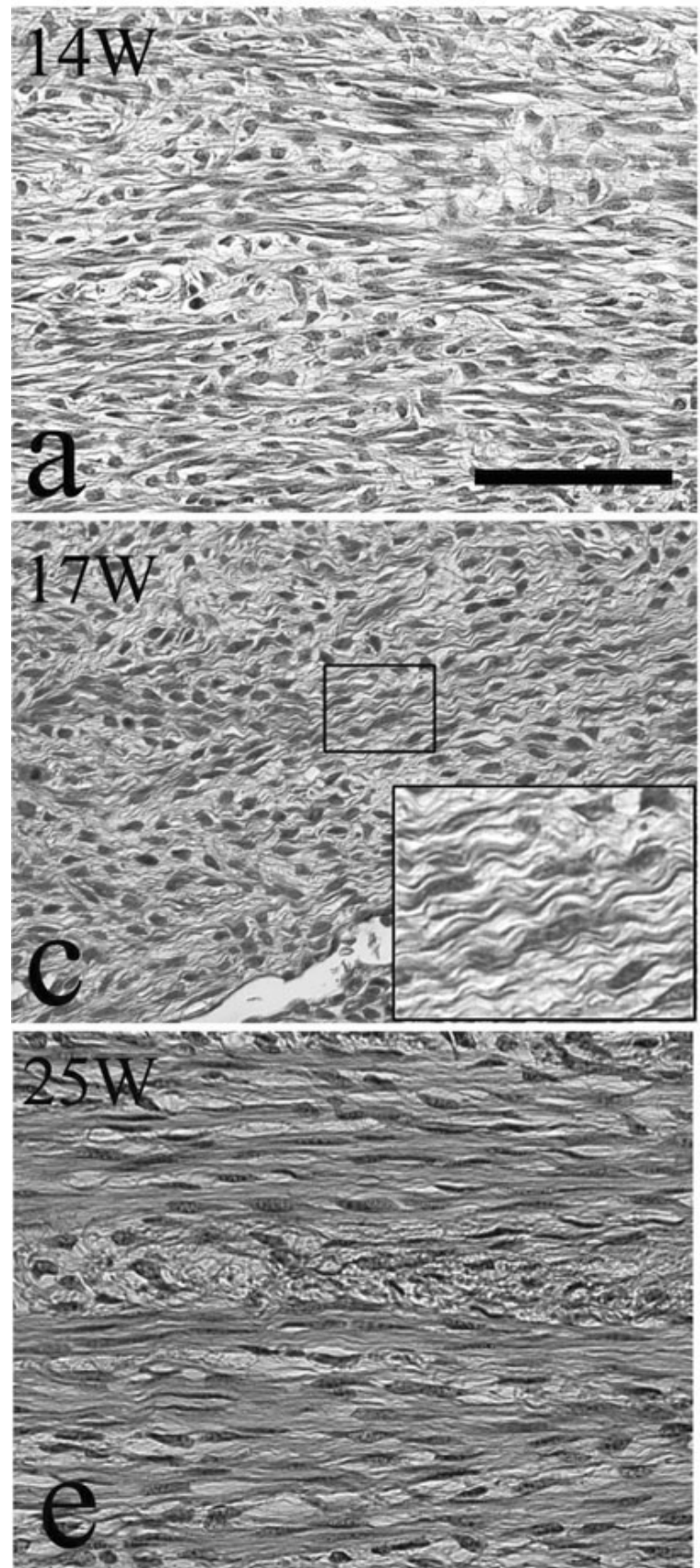

Figure 1-2. Histology of the meniscus in human fetuses. The anterior insertion of the fetal menisci from $14(\mathrm{a}, \mathrm{b}), 17(\mathrm{c}, \mathrm{d})$ and $25(\mathrm{e}, \mathrm{f})$ weeks of gestation. Bars, $100 \mathrm{~mm}$. HE staining. From Fukazawa ${ }^{40}$ with permission.

\section{Medial meniscus}
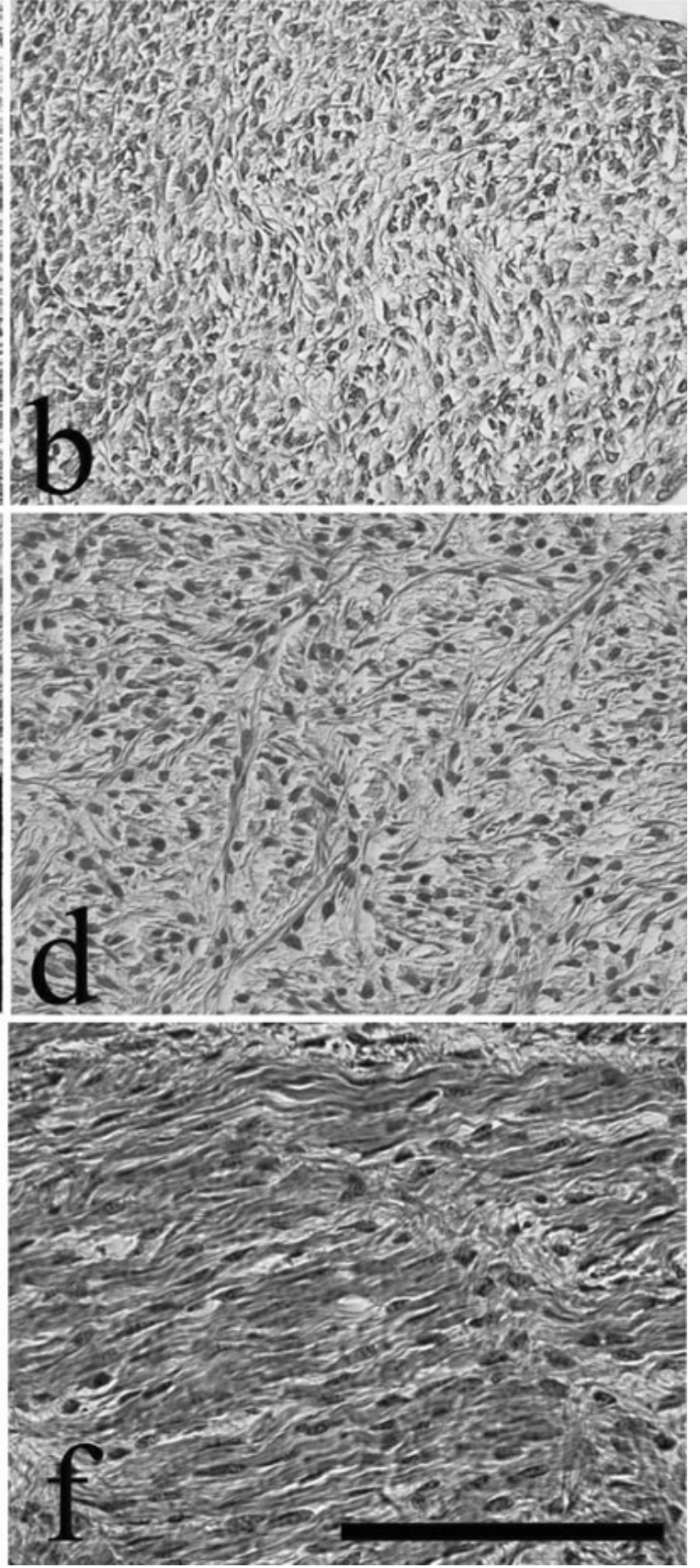
As the menisci develop in utero, the morphometry and relationship of this tissue to tibial plateau changes ${ }^{40,42}$. Specifically, the meniscus is highly cellularized with large nucleusto-cytoplasm ratios during prenatal development with blood vessels identifiable throughout the substance of fetal menisci ${ }^{44}$, although vasculature is most dense in the outer third region ${ }^{42}$. From prenatal to postnatal, there are no sharp changes in the morphometry of the menisci, but it progressively becomes less cellular, devascularized, and more collagenated (Figure 1-32 ). During adolescence, vasculature continues to move toward the outer third of the menisci, yet vessels can still be seen through the inner zones in the 10-11yr old meniscit ${ }^{42}$. However, in the mature adult, blood vessels are only found in the periphery of the menisci ${ }^{42,44}$. Proprioceptive characteristics of the menisci lie in the perimeniscal innervation as well, as large nerves associated with blood vessels circumferentially line the menisci ${ }^{44}$. Both anterior and posterior horns in adult menisci are greatly innervated as well, aiding with vasomotor control, identification of pain, and likely postural/protective muscular reflexes ${ }^{44,45}$.

Immobilization has been shown to play a critical role in the development and homeostasis of the knee joint soft tissues ${ }^{43,46-50}$. In chick embryos, immobilization does not influence meniscal condensation, but does result in meniscal cell degeneration and eventual meniscal disappearance during embryonic development ${ }^{43}$. In the mature lapine menisci, permeability has been shown to decrease following increased durations of immobilization ${ }^{50}$. Additionally, lapine menisci subjected to meniscal injury and subsequent immobilization demonstrated a reduced affinity for healing compared to remobilized animals ${ }^{51}$. However, traditional recommendations in rehabilitation following meniscal repair suggest 4-6weeks of decreased mobility ${ }^{52}$. Thus, the debate of whether or not to immediately remobilization or immobilization the knee following meniscal surgery is still clinically argued. 


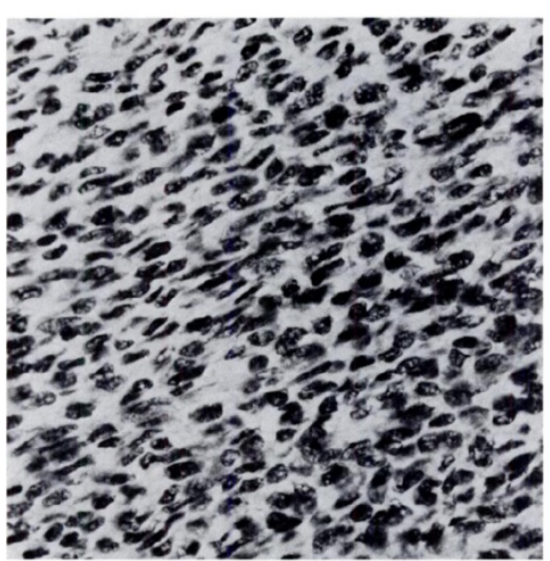

$\sim 14$ wk old fetus

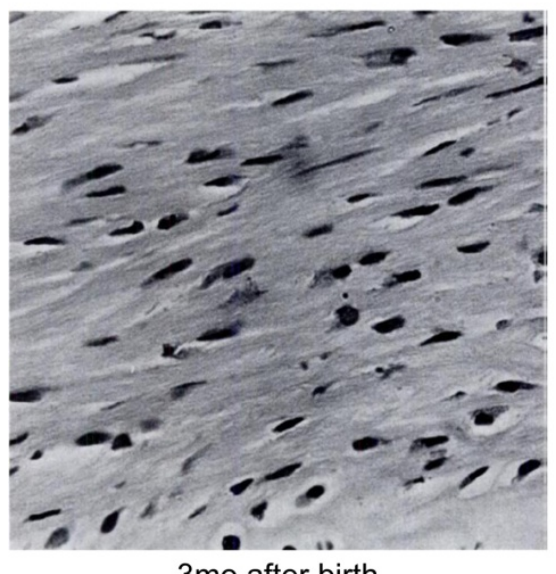

3 mo after birth

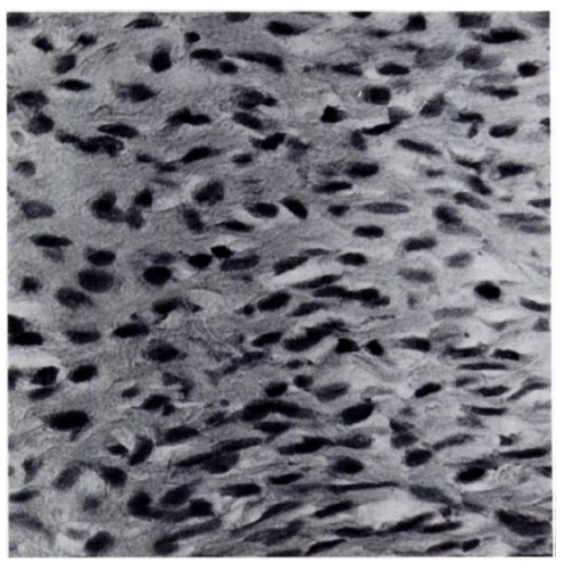

$\sim 30$ wk old fetus

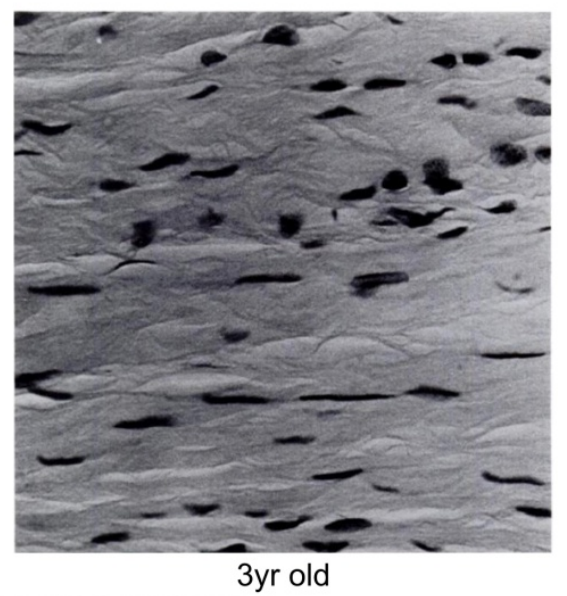

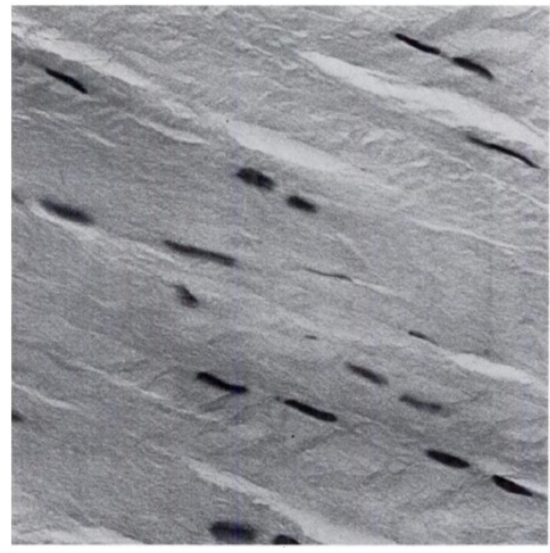

$10 y$ old

Figure 1-3. Photomicrographs of developing menisci (hematoxylin and eosin, $x 50$ ). Note the progressive decrease in cellularity and increase in intercellular matrix with increasing maturation. From Clark et al ${ }^{42}$ with permission. 


\section{Composition \& Structure of the Meniscus}

The menisci are $\mathrm{C}$-shaped, fibrocartilagenous tissue that lies between the femoral condyles and the tibial plateau. A coronal section of the meniscus is mostly triangular, with the outer zones being thicker than their inner counterparts (Figure 1-4). The superior surface, which provides a frictionless articulation for the femoral condyles, is concaveshaped, whereas the deep surface, resting atop the tibial plateau, is flat. The ends, or horns, are attached to the tibial plateau via ligamentous structures. These horns prevent the meniscus from moving too freely in the joint but also allow the meniscus to stretch radially during movement and loading.

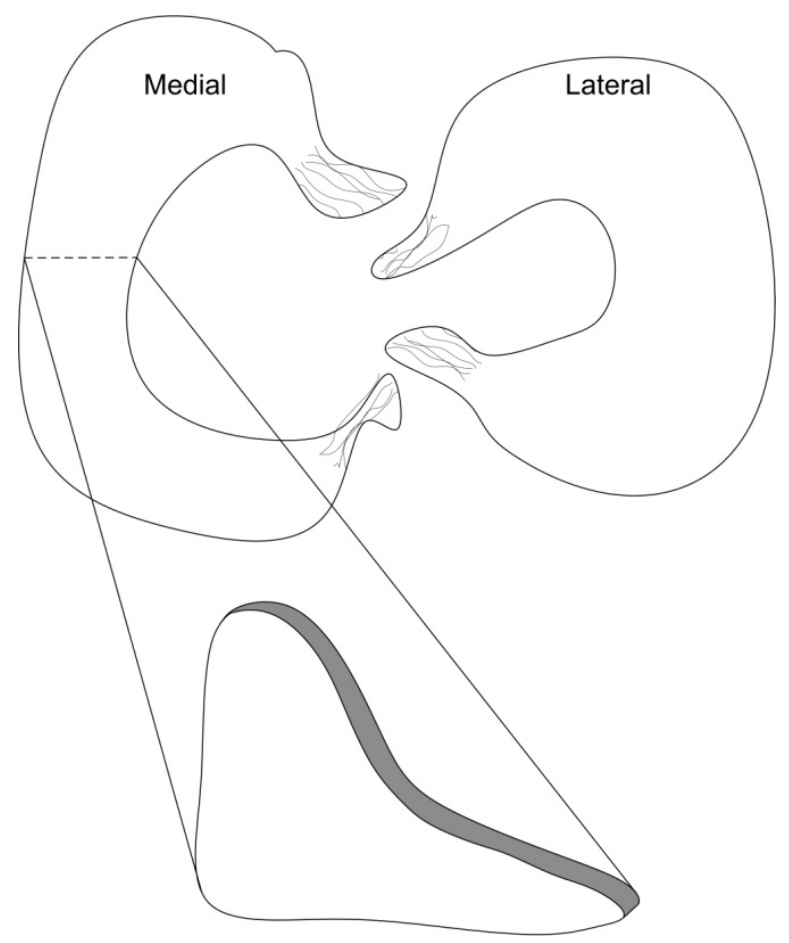

Figure 1-4. Schematic of medial and lateral menisci, with coronal cross-section.

The structure of the meniscus is created by the interaction, density, and orientation of type I and type II collagen and proteoglycans, which are the main constituents of the extracellular matrix. The outer region of the meniscus consists of radially-oriented type I collagen fibers, which provides tensile strength from hoop stresses during loading ${ }^{53}$. This region is vascularized and innervated ${ }^{54}$, providing an ability to heal itself upon injury. The inner region, subjected to compressive loads, contains nearly all of the type II 
collagen of the tissue ${ }^{53,55}$. The cellular density and phenotype vary throughout the menisci as well. A higher concentration of ovoid-shaped and fusiform cells, similar to chondrocytes, reside in the deep regions and inner zones of the tissue ${ }^{56,57}$. Spindle cells, similar to fibroblasts, are located on the outer regions and superior surfaces of the meniscus ${ }^{57}$. The generalized term "fibrochondrocytes" is often used to refer to the entire cell population of the menisci. However, recent work by Verdonk et al has characterized the phenotype of knee meniscal cells ${ }^{58}$. Three distinct hematopoietic (CD34-positive) cell populations are observed in adult human menisci, particularly in the outer vascular region (platelet endothelial cell adhesion molecule-1 [PECAM-1] negative), the superficial zone (PECAM-1 negative), and synovial sublining tissue regions (PECAM-1 positive) ${ }^{58}$. Recently, multi-lineage differentiation of fibrochondrocytes has been explored in order to better understand the healing capabilities of the meniscus, as progenitor activity may aid in migration, proliferation, and repair at injury sites ${ }^{59-62}$. The role of transforming growth factor- $\beta$ (TGF- $\beta$ ) on meniscal fibrochondrocytes has been shown to promote chondrogenesis in vitro ${ }^{59,} 62,63$. Fibrochondrocytes from immature bovine have demonstrated culture-dependent behavior ${ }^{62}$. Specifically, fibrochondrocytes have shown differentiating capabilities to adipogenic and osteogenic culture media ${ }^{64}$. Such behavior may depend on the region from which fibrochrondrocytes were derived ${ }^{62}$. Additionally, the presence of mesenchymal stem cells in the meniscus was recently reported and likely influences the differentiating capabilities of fibrochondrocytes ${ }^{65}$.

A key component of the menisci is proteoglycans. By binding, organizing, and retaining water molecules in the matrix of the meniscus, proteoglycans play a biophysical role in contributing to the tissue's biphasic characteristics ${ }^{66}$. Oscillatory fluid flow, as observed with water moving in and out of the matrix during walking, provides a mechanical environment that prompts meniscal cells to produce glycosaminoglycans via a calciumdependent pathway ${ }^{67}$. Proteoglycans consist of a large core protein with attached, negatively-charged, repeating sugar chains, called glycosaminoglycans. A detailed list of major proteoglycans and glycosaminoglycans of the menisci are listed in Table 1-1. 
The most abundant proteoglycan of the meniscus is aggrecan. Aggrecan is a macromolecule that remodels often and has well-characterized multi-domains that has been extensively studied for its role in articular cartilage ${ }^{68-70}$. The domain organization and structure of aggrecan is illustrated in Figure $1-5^{70}$. The aggrecan core protein itself is approximately $220 \mathrm{kD}$ in size, and the complex aggregated monomer with glycosaminoglycans link to a single hyaluronan backbone via the N-terminal globular domain $(\mathrm{G} 1)$ and link protein ${ }^{71}$. The link protein is analogous in structure to the G1 domain, and is responsible for linking the G1 region of aggrecan to the HA backbone ${ }^{72}$.
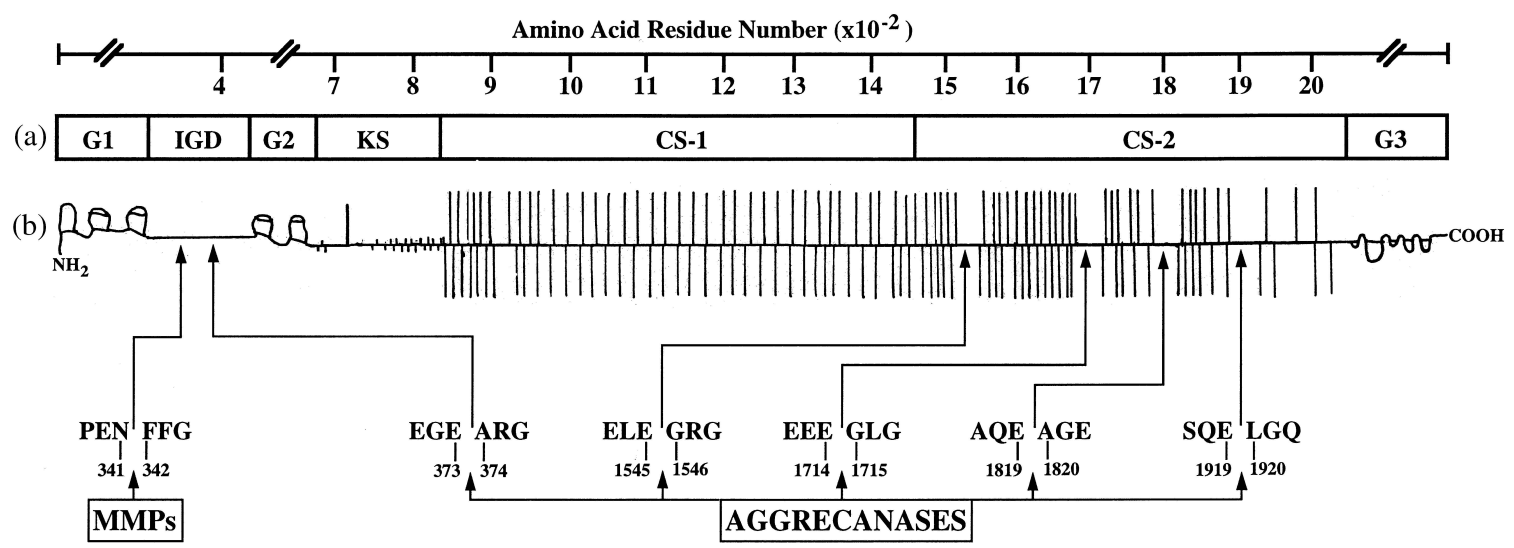

Figure 1-5. Schematic representation of the domain organization (a) and structure (b) of human cartilage aggrecan and depiction of the specific peptide bonds cleaved in situ in articular cartilage by aggrecanases or MMPs. Specific peptide bonds hydrolyzed in situ by MMPs or aggrecanases, and the position of these sites within the aggrecan core protein, are indicated by arrows. From Caterson et al ${ }^{70}$ with permission.

The interglobular domain (IGD), which is approximately 150 amino acids in length, attaches the G1 domain to the rest of the molecule ${ }^{71}$, and is subject to proteolytic catabolism potentially due to its structure ${ }^{71,73}$. Depending on the aggregation of the molecules, such a macromolecule could have a molecular weight of over 200 million $\mathrm{kD}^{69}$. Aggrecan has multiple cleavage sites (Figure 1-5), which can occur in the IGD or sulfate-rich regions, and can be cleaved by matrix metalloproteinases (MMPs) and $\operatorname{aggrecanases}^{70,71}$. Small leucine-rich proteoglycans (SLRP) also play important roles in the menisci ${ }^{74}$. Although aggrecan is evenly distributed throughout the menisci, SLRPs are more abundant in the inner two-thirds (avascular) zones ${ }^{75}$. Perhaps because of their small 
size, SLRPs are more resistant to proteolytic cleavage than aggrecan, and are known to interact with and protect collagen fibrils of cartilage ${ }^{76}$.

Table 1-1. Proteoglycans and glycosaminoglycans of the meniscus.

\begin{tabular}{|c|c|c|}
\hline Macromolecule & Structure & Ref \\
\hline \multicolumn{3}{|l|}{ Proteoglycans } \\
\hline Aggrecan & $\begin{array}{l}220 \mathrm{kD}-\text { consists of protein core, } \mathrm{CS} \text { and } \\
\mathrm{KS} \text { chains, link protein }\end{array}$ & 69 \\
\hline SLRPs & $\begin{array}{l}\text { Include fibromodulin, decorin, biglycan, } \\
\text { lumican, keratocan }\end{array}$ & 74 \\
\hline \multicolumn{3}{|l|}{ Glycosaminoglycans } \\
\hline Chondroitin 6-sulfate & $\sum_{\mathrm{NHCOCH}}^{2 \mathrm{OSO}_{3}}$ & $\begin{array}{l}55 \\
77\end{array}$ \\
\hline Chondroitin 4-sulfate & $\int_{\mathrm{NHCOCH}}$ & $\begin{array}{l}55 \\
77\end{array}$ \\
\hline Dermatan sulfate & $\underbrace{H}_{H} \underbrace{H}_{H N C O C H}$ & $\begin{array}{l}55 \\
78 \\
79\end{array}$ \\
\hline Keratin sulfate & $\mathrm{NHCOCH}_{3}$ & $\begin{array}{l}55 \\
77\end{array}$ \\
\hline Hyaluronic acid & 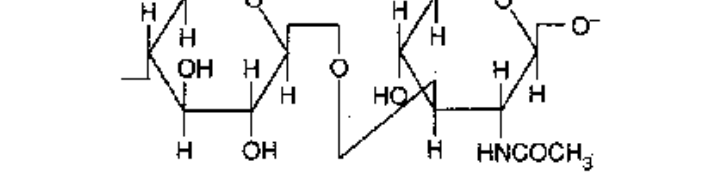 & 78 \\
\hline
\end{tabular}


Because the compressive properties of menisci are dependent on the fixed negative charge and sulfation of glycosaminoglycans on the aggrecan monomers ${ }^{80}$, the attachment sites of such glycosaminoglycan chains to the aggrecan core protein are of particular interest. Glycosaminoglycans are added to the aggrecan core protein intracellularly, although each glycosaminoglycan attaches to distinct locations by means of distinct set of glycosyl transferases ${ }^{69}$. Chondroitin sulfate (CS) transferases attach the linkage region to serine in the Ser-Gly sequence and elongate via glucuronic acid (glcA)- $N$ Acetylgalactosamine (galNAc) repeating disaccharide ${ }^{69}$. During elongation, sulfate esters become added to the 4- and 6- positions of the hexosamine residues. Upon elongation completion, galNAc residues cap the finished chains. Keratin sulfate (KS) also substitutes the aggrecan core protein, wherein KS can attach to both KS and CS regions. Glycosaminoglycan chains of KS are added to salactose residue on oligo-branches containing galactose $\beta 1$ (gal $\beta 1$ )- $N$-acetyl-D-glucosamine (4glcNAc) sequences. This chain is elongated by alternating gal and glcNAc residues and sulfated at the 6-position of galactose or $\mathrm{N}$-acetylglucosamine. The finished chains are capped with sialic acid ${ }^{69}$. Along with the larger proteoglycans like aggrecan, smaller constituents, such as decorin $(70 \mathrm{kD})$ and biglycan $(100 \mathrm{kD})$, are present in menisci as well ${ }^{69}$. These small proteoglycans in cartilage contain dermatin sulfate (DS) chains as opposed to CS or KS chains, and have alternating idoA- and glcA- containing disaccharide repeats ${ }^{69}$. Another small proteoglycan in soft tissues is fibromodulin $(30 \mathrm{kD})$, substituted with only a few KS chains.

The distribution of proteoglycans and glycosaminoglycans in both cartilage and menisci has been demonstrated in the literature ${ }^{81-83}$. Aggrecan is expressed more in the inner zone of the meniscus ${ }^{53}$. In skeletally mature rabbits, biglycan is found throughout the meniscus, whereas decorin is found on the periphery of cartilaginous structures ${ }^{84}$.

The integration of woven collagen fibers and proteoglycans allow the meniscus to compress and distribute load to the tibial plateau during weight-bearing activities and return its shape following unloading. 


\section{Mechanics of the Knee Meniscus}

The shape, compressive and tensile properties, and biphasic nature of the meniscus contribute to its load distribution, lubrication, shock absorption and joint alignment characteristics $^{85,86}$. The meniscus attaches primarily to the tibial plateau, providing the tissue with unique structural properties. The alignment and structure of the peripheral collagen fibers in the outer third of the meniscal body (known as the red zone because of its vasculature nature) support hoop stress in order to aid alignment and distribute loads during mobility. The attachments prevent meniscal extrusion and maintain stress distributions of the femur on the body of the meniscus, instead of allowing the meniscus to displace, exposing the cartilage of the tibial plateau. The inner regions of the menisci (white zone due to its avascular nature) provide the tissue with compressibility and are cartilage-like in cell phenotype and matrix structure.

The dynamic behavior of the meniscus within the joint is not well understood. The meniscus can be considered an anisotropic ${ }^{87}$, inhomogeneous ${ }^{80}$, non-linear ${ }^{79}$ biphasic material in which the solid matrix and the interstitial fluid of the meniscus dictate the response of the tissue to compressive loading. The matrix component of biphasic materials, such as articular cartilage, has been modeled as an incompressible solid consisting of collagen fibers, proteoglycans, and chondrocytes ${ }^{88}$. The interstitial fluid is modeled as an incompressible liquid ${ }^{88}$. The stress observed in soft tissues is governed by the interstitial pressure as it relates to the fluid and solid phases, known as the biphasic mixture model (Equation 1-1):

$$
\sigma=-p I+\sigma^{e}
$$

Equation 1-1 ${ }^{88}$

where $p$ is the interstitial pressure, $\mathbf{I}$ is the identity tensor (together, modeling the liquid phase), and $\sigma^{e}$ is the effective elastic stress (modeling the solid phase).

The Conewise Linear Elastic theory, when applied to articular cartilage, has been successfully shown to predict experimental results in confined and unconfined compression as well as torsional shear ${ }^{88}$. This theory accounts for nonlinearities in the tissue's tensile and compressive behavior as well as the depth-dependence of material 
properties $^{88}$. Others have used a fibril-reinforced poro-viscoelastic model of the menisci to simulate joint stresses in healthy and arthritic knees ${ }^{89}$. This model subdivides the biphasic mixture model further, separating the solid phase into fibrillar and non-fibrillar components $^{89}$.

Biphasic theory can also be applied to nanoscale modeling of biological materials ${ }^{90,91}$. Nanoindentation has recently been shown to be a useful tool in understanding the changes in mechanical properties across biologic transition regions, and how such changes in properties may be associated with mineralization, collagen organization, and microscopic 2-D structure ${ }^{90,92-94}$. Use of both macro- and micro/nano-scale mechanical testing in the future will further advance our understanding of the behavior of the menisci.

The spatial distribution of contact pressures on the meniscus have been demonstrated in the literature ${ }^{30,95}$. It is well known that removal of the meniscus results in decreased contact area of joint loads caused by the femoral condyles articulating directly onto the tibial plateau ${ }^{30}$. This results in excessive stresses on the articulating surfaces, increasing the wear characteristics and ultimately leading to cartilage degradation and osteoarthritis. Although common surgical techniques have been employed for many years to remove and repair meniscal tears, clinical follow-up studies have very weakly proven their benefit.

\section{Meniscal Tears}

Twisting of the knee, rupture of the anterior cruciate ligament (ACL), or degeneration of the meniscus can result in tear formation, progression, and reduced efficacy of the meniscal function (Figure 1-6). Longitudinal vertical tears are typically observed following traumatic injury, whereas horizontal and oblique tears are often a result of degenerative/age related changes to the menisci ${ }^{4}$ (Figure 1-6). The derivation of radial tears is not fully understood ${ }^{4}$. Symptomatic osteoarthritis (OA) is typically associated with the degenerative progression of tears ${ }^{4}$. Meniscal injuries are typically treated 
arthroscopically, with approximately 636,000 arthroscopic surgeries per year ${ }^{96}$. Surgeons typically resort to removing $25-100 \%$ of the meniscus ${ }^{96}$ or suture an existing tear using materials such as poly-L-lactic-acid or polydioxanone ${ }^{97}$. However, removal of the entire meniscus results in higher stress concentrations on the remaining meniscus and underlying tibial plateau ${ }^{30,98}$. This has been suggested to lead to the deterioration of articular cartilage and the onset of osteoarthritis $(\mathrm{OA})^{95,}$,99-101. The progression of OA after meniscectomy has been associated with the increased contact pressures on the cartilage due to reduced meniscal area ${ }^{30}$. Additionally, the presence of tears, as well as the removal of torn regions of menisci, leads to proprioceptive deficits. Therefore, encouraging the healing of menisci is important, and minimal removal of the damaged tissue has been more recently advised ${ }^{102}$. Nonetheless, nonsurgical therapies may not be enough, and certain tear types may eventually result in problems. For example, in the event that a longitudinal tear does not heal or is not fixed, it may progress into a buckethandle tear that can displace in the joint and lead to severe pain and joint locking ${ }^{103}$. Thus, understanding the mechanics, healing capacity, and treatment remedies for meniscal injuries is at the forefront in order to maintain and improve ${ }^{103}$ knee joint health.

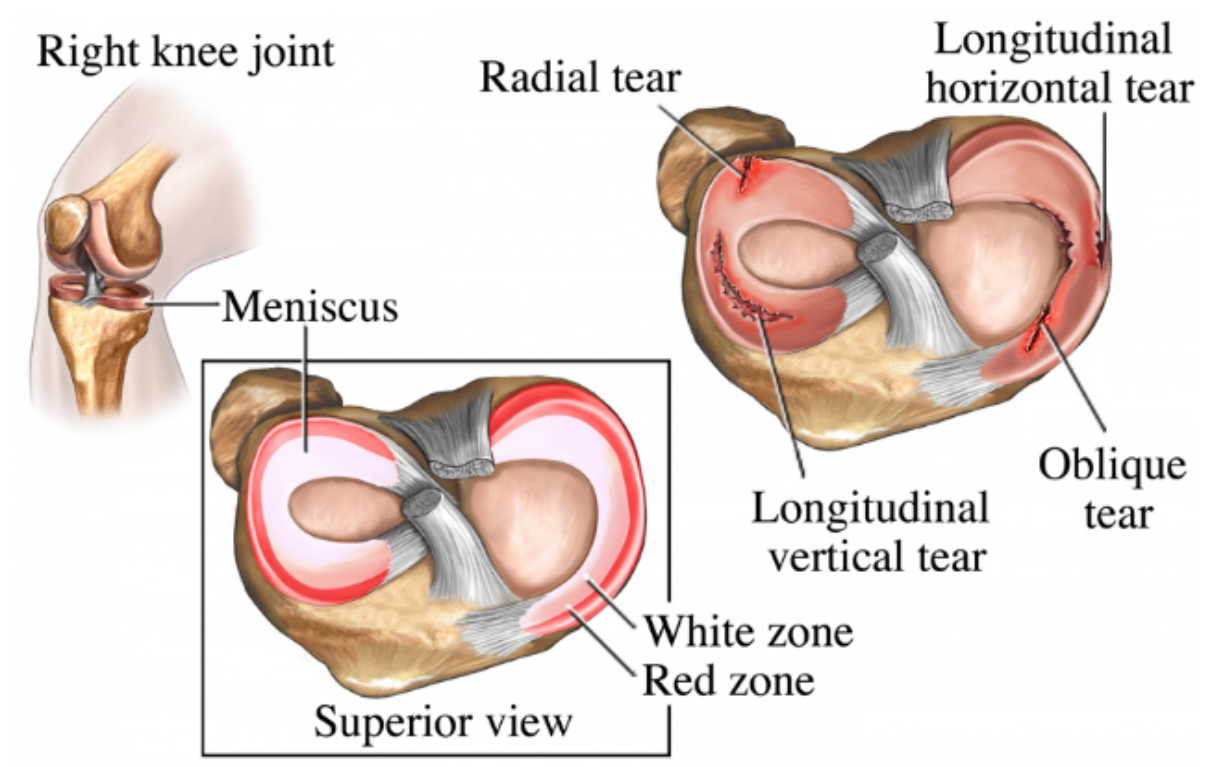

Figure 1-6. Potential tear types and red/white zones of the menisci. From Nucleus Medical Media, Inc with permission. 


\section{Mechanotransduction of the Meniscus}

Cellularized tissues can be influenced by changes to their mechanical environments. The effect of mechanical stimulation on cellular signaling and activity is known as mechanotransduction. Such responses lead to variations in production of matrix protein and enzyme production, which in turn influences the structural makeup of the tissue. A healthy, normal mechanical environment likely contributes to the constant matrix turnover, or homeostasis, of soft tissues ${ }^{3}$. However, a detrimental mechanical environment may lead to overproduction of catabolic molecules which degrade the matrix in which the cell resides ${ }^{104}$. The expression and production of these molecules may be initiated via integrin signaling or other intracellular pathways ${ }^{105,}{ }^{106}$. The construction and maintenance of the extracellular matrix is dependent on the health of the cell ${ }^{106}$.

Just as bones require loading to prevent atrophy, so too do the meniscus and articular cartilage $^{107-109}$. In an extensive review article, Griffin and Guilak discuss many in vitro and in vivo experiments that have demonstrated the need for dynamic compression and joint loading on the health of articular cartilage ${ }^{3}$. It has been demonstrated that rats have an apparent increase in meniscal collagen and proteoglycan content following exposure to treadmill exercise ${ }^{110}$. Similarly, in rabbits exposed to antigen induced arthritis, continuous passive motion of the meniscus has been demonstrated to significantly promote anti-inflammatory mediation of cytokine activity, such as reduced cyclooxygenase-2 (COX-2), matrix metalloproteinase-1 (MMP-1), interleukin-1 (IL-1) and -10 activity, after just as little as 24 hours exposure ${ }^{109}$. Oscillatory fluid flow has been found to induce shear stresses on meniscal cells, which can contribute to increased intracellular calcium-mediated production of sulfated glycosaminoglycans ${ }^{67}$. In an in vivo lapine model, surgically-induced meniscal tears of the outer region demonstrated an increase in blood flow and vascular volume with mobilization. In the same study, rabbits subjected to hind limb immobilization after meniscal tears had an observed increase in angiogenesis only. This injury model demonstrated the vascular response of the meniscus after injury, which contributes to the tissue's healing ability. Shin et al. demonstrated an increased 
synthesis of protein and sulfated glycosaminoglycans in meniscal explants following dynamic compression exercises of $24 \mathrm{~h}^{111}$. This study also demonstrated that mechanical stimulation "overrides" glycosaminoglycan and protein production otherwise inhibited by exogenous IL-1.

Whereas unloading and disuse can be detrimental to knee joint health, overloading may also lead to compromised integrity and deleterious function of menisci and cartilage. Kessler et al. investigated the effect of running on meniscal and articular cartilage thickness ${ }^{112}$. The gross regression of meniscal and articular cartilage thickness immediately following running was attributed to the tissues' biphasic material properties, and the rate of recovery to original thickness after an hour was suggested to be a factor of the tissues' permeability characteristics. However, it is still inconclusive if long-distance running is detrimental to the health of the meniscus. In vitro investigations of cyclic mechanical strain on meniscal cells have demonstrated an alteration in protein, proteoglycan, nitric oxide (NO) and prostaglandin- $\mathrm{E}_{2}$ (PGE-2) production ${ }^{113}$. Cyclic biaxial strain produced increases in NO and PGE-2 after 24h exposure. However, an anabolic effect of mechanical loading was also observed, with increased production of proteoglycan and protein. Using meniscal explants, Upton et al. also observed a mechanical response of fibrochondrocytes ${ }^{114}$. After exposure to a static compressive load of $0.1 \mathrm{MPa}$ for $24 \mathrm{~h}$, a decrease in decorin and type I collagen gene expression, as well as an increase in MMP-1 gene expression, was observed. Following dynamic compression of explants (ranging from $0.08-0.16 \mathrm{MPa}$ stresses at $0.5 \mathrm{~Hz}$ ) for $24 \mathrm{~h}$, decorin gene expression significantly decreased. Fink et al. also used meniscal explants to investigate the effect of dynamic compression on nitric oxide production of the meniscus ${ }^{56}$. Dynamic compression of $0.1 \mathrm{MPa}$ for $24 \mathrm{~h}$ significantly increased NO production in both medial and lateral meniscal explants when compared to uncompressed explants. Also, dynamically compressed meniscal explants expressed NO in a regional-dependent manner, with surface explants expressing more NO than their deep counterparts. This anatomical dependence may have been due to the cellular density distribution of the meniscus, with more cells in the superior zone able to produce more NO. These in vitro investigations 
bring light to the mechanical influence on fibrochondrocyte activity. However, a major shortcoming of these experiments exists in the duration of the experiments, as it is not physiological to cyclically load the meniscus for a constant $24 \mathrm{~h}$ period. Also, differential stress magnitudes may be present on the meniscal surface in vivo that were not controlled for with these experiments. Using a shorter loading exposure duration and different loading regime, Ferretti et al. recently investigated the effect of $4 \mathrm{~h}$ of dynamic tensile forces on the expression of MMP-13, iNOS, and tumor necrosis factor- $\alpha$ (TNF- $\alpha$ ), as well as its inhibition on IL-1 $\beta$-induced cytokine expression, on fibrochondrocytes ${ }^{115}$. Fibrochondrocytes exposed to dynamic tensile forces observed a significant decrease in both TNF- $\alpha$ and MMP-13 expression compared to unloaded cells, which was dependent on the magnitude of tensile force. Dynamic tensile forces are observed in the outer region of the meniscus, and the influence of this mechanical environment on inhibition of IL-1 $\beta$-induced NO, TNF- $\alpha$, and MMP-13 are significant findings. Investigations must be performed on physiological mechanical loading of meniscal tissue.

As previously mentioned, mechanical loading on the meniscus and articular cartilage regulates the amount of collagen and proteoglycans produced by these tissues; however, the mechanisms of regulation have not been extensively explored ${ }^{15,29,68,107,116}$. Djurasovic et al. investigated the effect of immobilization of beagle hind limbs on gene expression in the meniscus over a period of four weeks ${ }^{68}$. In their study, they found that aggrecan gene expression significantly decreased in the immobilized limb, with the largest decrease in the posterior region of the lateral meniscus. Sulfated glycosaminoglycan (S-GAG) also decreased in the immobilized meniscus and water content increased. The biophysical dependence on loading is apparent in the maintenance of proteoglycans of the meniscus. Higher expression of aggrecan and S-GAG in nonimmobilized limbs for this study may be attributed to excessive, compensatory loading on the weight-bearing limbs; hence, more controlled experimental procedures utilizing animals without immobilization should also be investigated. Hennerbichler et al. recently investigated the effect of dynamic compression at different stresses on meniscal explants from inner and outer zones ${ }^{117}$. They noted an increase in prostaglandin- $\mathrm{E}_{2}$ 
(PGE-2) and nitric oxide (NO) production from inner zones compared to outer zones, when compressed at different stress magnitudes. Both PGE-2 and NO are proinflammatory mediators, which are considered to contribute to cytokine recruitment. Such recruitment may lead to a disruption in molecular homeostasis, which may affect the response of the tissue to loading.

The use of finite element techniques has contributed to understanding how mechanical loading affects the meniscus. Gupta and Haut Donahue recently discussed how anatomical location may influence the material properties of the tissue, wherein influencing the behavior of local cells ${ }^{118}$. The stresses acting on the cells were found to differ compared to the surrounding extracellular matrix. Also, the shielding effect of surrounding pericellular matrix material was influenced by the shape of these cells. In articular cartilage, investigations in localized forces, cellular permeability and material property alterations caused by disease have been explored using finite element methods ${ }^{119,120}$. However, it is imperative to further investigate the role of pericellular microenvironments on meniscal cells, as the tissue is much different than that of articular cartilage. Altered loading, caused by forfeited integrity of the meniscus, has been examined using finite element methods, as well. Zielinska and Haut Donahue investigated the distribution of contact pressures on the surface of the meniscus, as well as the relevant strain observed by the tissue, when modeled as a linear elastic, transversely isotropic material ${ }^{95}$. This model provided a representation of contact pressure during standing when loaded at two-times body weight. A noticeably greater contact pressure was observed in the posterior region compared to the anterior and central regions of the medial meniscus, with uniformity of contact pressures in the lateral meniscus. Using a meniscectomy model, the researchers observed that axial strain increased as more tissue was removed, with up to nearly $18 \%$ axial strain after removal of $30-60 \%$ of the meniscus. 


\section{Molecules Involved in Soft Tissue Degradation}

It has been previously shown that varying levels of dynamic strain can influence the biochemical behavior of menisci in vitro ${ }^{32}$. Additionally, there is strong evidence that cellular activity of soft tissues plays an important role in the progression of osteoarthritis. Pro-inflammatory mediators, such as Interleukin-1 (IL-1), have been detected in osteoarthritic synovium and cartilage ${ }^{31}$. IL-1 is a $17 \mathrm{kDa}$ polypeptide that promotes leukocyte infiltration, prostaglandin synthesis, joint swelling, and tissue destruction ${ }^{121}$. IL-1 is produced in two distinct polypeptide forms; IL- $1 \alpha$ and IL-1 $\beta$. The IL-1 family also includes IL-1 receptor antagonist protein (IL-1RA), which prevents IL-1 from binding to cell surface receptors. Although IL-1 $\alpha$ and IL-1 $\beta$ have similar roles in physiological activity, they function differently. IL-1 $\alpha$ is expressed as a membrane-bound protein and IL- $1 \beta$ is soluble $\mathrm{e}^{121,122}$. Both IL- $1 \alpha$ and IL-1 $\beta$ initiate collagenase activity and decrease pain tolerance, and many approaches in research attempt blocking these deleterious cytokines. The use of IL-1RA and other molecules have been used to reduce IL-1 activity in vitro and in clinical trials ${ }^{123-125}$.

The downstream effects of IL-1 on the expression and production of other molecules has also been readily studied. IL-1 has been shown to increase nitric oxide (NO) levels, as well as increasing production of MMPs, lactate and prostaglandin- $E_{2}$ (PGE-2), all of which contribute to degradation of meniscal and other fibroblastic tissue ${ }^{104,126,127}$. The addition of IL-1 to meniscal explants in vitro has been demonstrated to disrupt matrix turnover $^{111}$, and its inhibitory influence on mechanically stimulated explants is suggested to be dependent on nitric oxide synthase-2 (NOS2) gene expression and NO. The activity levels of MMPs and aggrecanases are also strongly influenced by IL-1 ${ }^{105,128}$, and such activity leads to cleavage of aggrecan, collagen, and other matrix molecules ${ }^{105}$ (Figure 1-7). The fragmentation associated with cleaved collagen and aggrecan promotes further irritation of surrounding cells, compounding the inflammatory response, promoting synovitis $^{129}$ and encouraging macrophage activation ${ }^{105}$. 


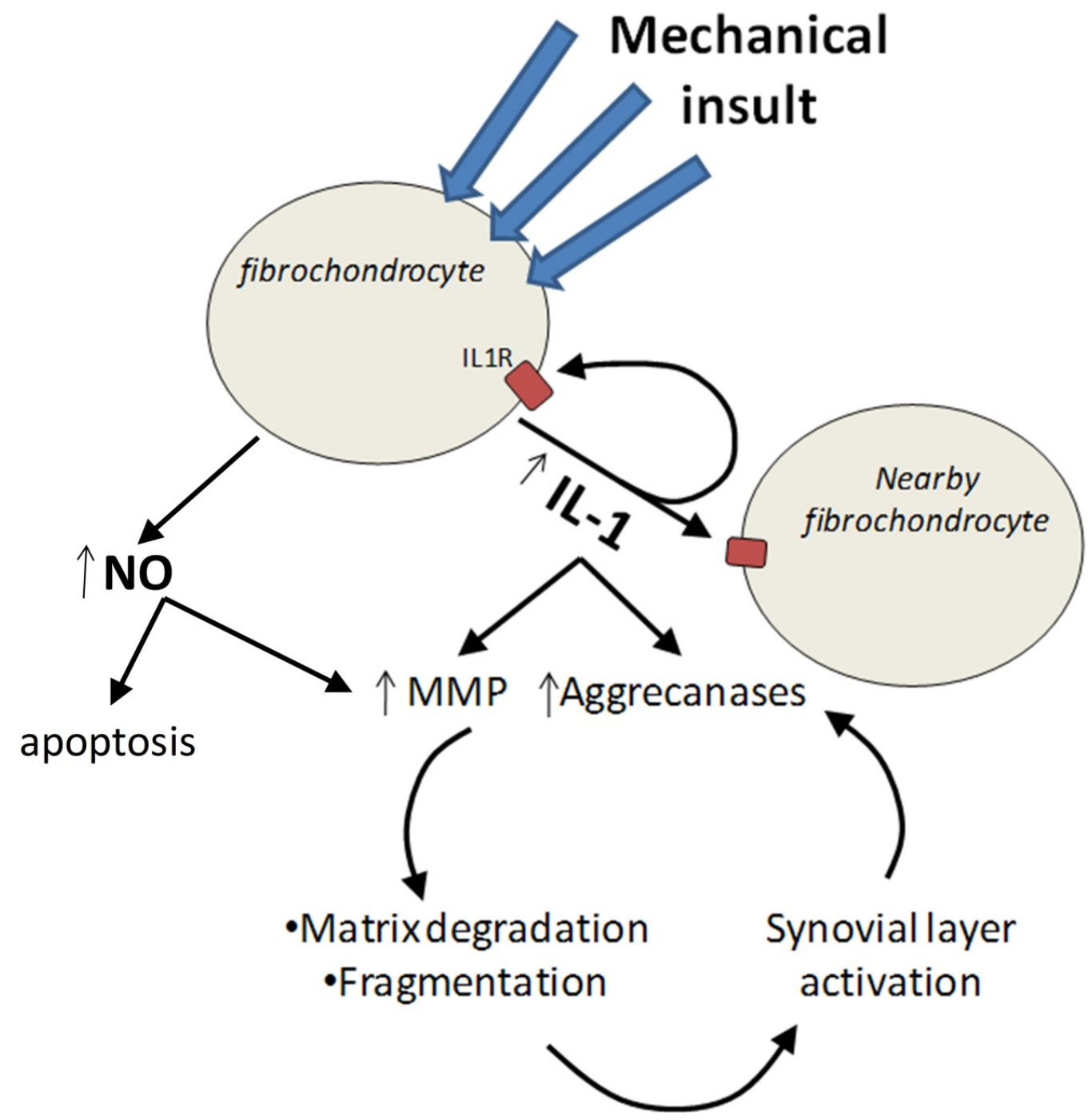

Figure 1-7. Catabolic biochemical pathway induced by compressive loading. IL1R represents the IL-1 receptor site. Thin arrows represent increase expression and production. Modified from Burrage et $\mathrm{al}^{105}$.

Aggrecanases and MMPs are responsible for cleaving specific matrix proteins, which leads to the degradation of soft tissues both in vitro and in vivo. The following describes secreted MMPs and their target molecules. MMP-1 and MMP-13 are collagenases, which degrade native collagen fibers ${ }^{130}$. Some of the cleavage sites of collagen incurred by MMP-1 include Gly784-Ile785 and Gly781-Ile782 ${ }^{131}$. Collagen type II is cleaved in vitro by MMP-13 at the Gly775-Leu776 site ${ }^{132}$. Aggrecan is also subject to MMP-13 cleavage, where cleavage in the IGD is observed regardless of the presence of $\mathrm{CS}^{133}$. MMP-3, of 
the stromelysin group, is responsible for degradation of proteoglycans and type IX collagen. MMP-9 is a gelatinase, responsible for degrading denatured collagen, proteoglycans, and fibronectin. Aggrecanases-1 and -2 (A Disintegrin and Metalloproteinase with ThromboSpondin motifs [ADAMTS]-4 and -5) are responsible for cleaving aggrecan specifically. These molecules differ from ADAMs (membranebound proteins) because ADAMTS lack a membrane-spanning region at their Cterminus $^{70}$. The specific cleavage sites of aggrecan by ADAMTS catabolism differ depending on the molecule of interest. Specifically, ADAMTS-4 (aggrecanase-1) cleaves aggrecan in vitro in the interglobular domain between Glu373-Ala374, and ADAMTS-5 (aggrecanase-2) cleaves aggrecan in the interglobular domain between Glu1871Leu1872 $134-136$.

Regular matrix turnover is essential for maintenance of healthy menisci. However, over expression and production of catabolic molecules may lead to matrix deterioration and meniscal destruction. Previous investigations have demonstrated an increase in catabolic gene expression of meniscal explants subjected to a single bout of overloading levels of dynamic compressive strain $^{32,134,135}$. The use of exogenous catabolic molecules leads to a decrease in the ability for soft tissues to repair and heal as well ${ }^{99,136-140}$. The presence of NO in synovial joints of those afflicted with OA is typically higher than those with healthy joints. Murrell et al. found that chondrocytes of articular cartilage produce more NO following infection or inflammation in vitro than ligaments ${ }^{141}$; however, their research did not investigate the NO production of cells of the meniscus. NO has been shown to inhibit aggrecan synthesis, enhance MMP activity, and is suggested to increase oxidative injury to chondrocytes and mediate chondrocyte apoptosis ${ }^{31,104}$. Hashimoto et al. determined apoptosis and NO production of lapine meniscal cells following ACLtransection ${ }^{104}$. It is interesting to note that, although they harvested menisci 9 weeks following injury, apoptosis of the meniscus was still significantly greater for injured knees; apoptosis typically initiates 6h-7 days immediately following injury in chondrocytes $^{142}$. This study used the contra-lateral, uninjured limb as the control limb, and this limb was not subjected to sham operation. It is unclear if compensatory or 
surgical effects influenced apoptosis of the operated meniscus. However, histological grade of meniscal degradation was correlated with percent apoptosis. Takahashi et al. investigated the effect of hyaluronan on NO production in a rabbit osteoarthritic model in $v_{i v o}{ }^{12}$. The meniscus was observed to produce significantly more NO than synovium, and the production of $\mathrm{NO}$ was significantly reduced by the addition of hyaluronan for both synovium and meniscus. However, hyaluronan did not significantly inhibit meniscal production of NO in the presence of lipopolysaccharide in vitro. Lipopolysaccharide is known to lead to expression and release of cytokines such as TNF- $\alpha$ and IL- ${ }^{143}$.

Inhibiting the catabolic IL-1 pathway induced by mechanical loading could potentially provide therapeutic benefits following traumatic injury. By blocking the IL-1 signaling pathway, catabolic damage may be held idle. Glucocorticoids have been shown to inhibit IL-1 production by making mRNA products unstable ${ }^{144}$. Additionally, corticoids like methylprednisone have been shown to reduce the number of mast cells in OA patients ${ }^{145}$. When used as a treatment for patients with OA, glucocorticoid therapy has been shown to reduce pain for up to four weeks ${ }^{146}$. Glucocorticoids are reportedly prescribed by rheumatologists for up to $95 \%$ of their patients ${ }^{146}$, yet their long-term efficacy has yet to be proven ${ }^{145}$. Organisms naturally control activation of IL-1 signaling via IL-1 receptor antagonist (IL-1RA), which binds to the receptor type I (IL-1RI) on the cell membrane and blocks signaling of secondary mediators such as NO and other cytokines. Researchers have successfully used IL-1RA to reverse the influence of detrimental mechanical compressions on cartilage destruction ${ }^{147,148}$. In vivo, intervertebral disks treated with IL-1RA demonstrated reduced cartilage lesions, synovitis, and osteophyte size $^{149}$. Anakinra, a recombinant form of IL-1RA, has been recently implemented as a clinical treatment for patients with symptomatic and radiographic knee $\mathrm{OA}^{150}$, and shows promise.

\section{Models for Investigating Knee OA}

Within the last decade, researchers have developed impaction-based models to investigate the role of microcracks and fissuring on soft tissue viability. In 2001, Ewers et 
al showed that, in vitro, impaction of cartilage that led to surface fissuring resulted in an increase in cell death at the fissure edge ${ }^{151}$. Additionally, Lewis et al discovered an increase in cell death at the fissure surfaces following cartilage impaction ${ }^{152}$. Although these works have been performed in vitro, they suggest the potentially damaging acute response of soft tissue to impaction, which should be further investigated.

For nearly 30 years, investigators have been studying the role of altered loading and impaired biomechanics using animal models. The most prominent and well-developed animal model for investigating knee $\mathrm{OA}$ has been the anterior cruciate ligament transection (ACLT) model $^{10,}{ }^{153-161}$. The ACLT model traditionally consists of a parapatellar incision to expose the joint space, and a clean scalpel incision to transect the ACL is performed. The joint space is then sutured closed and the animal treated with pain medication for 24-48hrs and antibiotics. With the ACLT model, researchers have investigated several different time points and morphological characteristic changes to the cartilage, subchondral bone, and menisci (Figure 1-8). Typically, researchers have investigated the damage to knees at least 2-3weeks after subjection to ACLT 10, 156, 161 . Within the first two weeks following ACLT of the rabbit knee, changes to the menisci have not been identified ${ }^{159}$. However, after only two weeks in the knees of rats subjected to ACLT, cells of the tibial articular cartilage stain positive for osteoclast markers (tartrate-resistant acid phosphatase, TRAP) ${ }^{156}$. Such damage to the knee has also led to an increase in the secretion of degradative molecules such as serum cartilage oligomeric matrix protein (COMP) and urinary C-telopeptide of type II collagen I and II (CTX-I and -II) ${ }^{156}$. In the menisci, an increase in water retention ${ }^{10}$, decrease in DNA/RNA content, altered expression of both catabolic and anabolic matrix molecules ${ }^{161}$, and changes in cell morphology ${ }^{159}$ have been observed three weeks after ACLT, most noticeably in the medial menisci(Figure 1-8). Four weeks after ACLT is still considered the early phase of disease ${ }^{155}$. An increase in apoptotic signaling in the articular cartilage has been observed at this time point ${ }^{155,157}$, and the release of nitrites has been linked to the increased density of apoptotic cells as well as the advanced degree of arthritic degeneration ${ }^{155}$. Fissures and erosion of articular cartilage has been observed just four weeks after ACLT ${ }^{162}$, and a 
greater density of osteophyte formations, the accumulation of hypertrophic cells, and an increase in nitrotyrosine in the articular cartilage has also been reported ${ }^{163}$. The formation of new blood vessels has also been observed, indicating the progression of OA in the ACLT joint after just four weeks ${ }^{163}$. After eight weeks, degradation of cartilage and menisci ensue ${ }^{12,154,158,163,164}$. For example, the menisci have been shown to produce more nitrites nine weeks after ACLT compared to the synovial tissue ${ }^{12}$. At this time point, the articular cartilage of the tibial plateau has become mostly osteophytic, and the chondrocytes have developed hypertrophic characteristics, expressing vascular endothelial growth factor (VEGF) ${ }^{163}$. As time progresses, ACLT continues to advance knee joint arthritis. After twelve weeks, the articular cartilage has been reported to demonstrate nitrotyrosine-positive cells in the entire hypertrophic and calcified regions, and apoptosis has been observed of all cells in the hypertrophic region ${ }^{163}$. Severe, advanced OA changes have been morphologically reported as wel1 ${ }^{164}$. Interestingly, the severity of meniscal damage in the ACLT knee of canines has not always been correlated with articular cartilage degeneration ${ }^{158}$. Additionally, in ACLT models, previous researchers focus mostly on the medial meniscus and how it responds to altered joint kinematics via cellular, molecular, and morphological changes ${ }^{10,159,161}$.

Investigations of changes to articular cartilage and menisci within the first few weeks of ACLT have only marginally been investigated ${ }^{159}$. One reason for this may be that altered loading mechanics do not lead to changes in menisci and cartilage morphologies indicative of degeneration. Another reason may be that surgically induced swelling and inflammation may not be recovered within an acute period following ACLT. However, such acute inflammation may confound the biochemical behavior within the knee and such surgically induced inflammation may not demonstrate an isolated, altered loading behavioral response of the sensitive soft tissues. Therefore, it is important to develop a more realistic model to investigate traumatically induced OA that will aid in translational therapies from bench-top to clinic. 


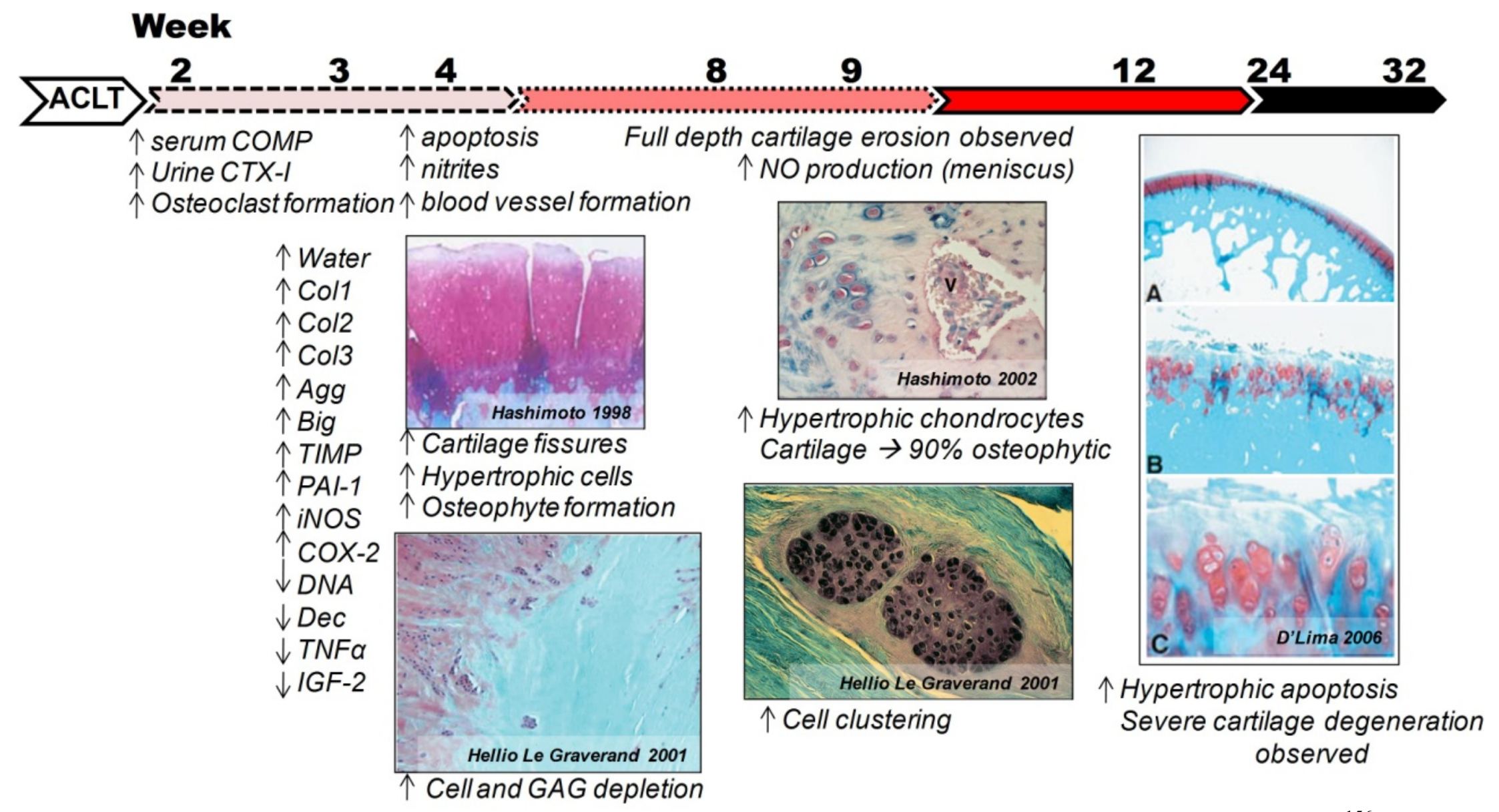

Figure 1-8. Timeline of degradation following ACLT. Week 2 changes were observed in using a rat ACLT model ${ }^{156}$, whereas all subsequent degenerative changes were observed using a rabbit ACLT model ${ }^{10,12,154,155,157-159,161-165}$. Images used with permissions. 


\section{Problem Statement}

\section{Catabolic Behavior Following Mechanical Loading of the Meniscus}

Mechanical loading of some musculoskeletal tissue, such as articular cartilage, has been shown to regulate the amount of collagen and proteoglycans, although mechanisms of such regulation have not been explored in the knee joint meniscus ${ }^{107,166,167}$. Meniscal lesions have been suggested as a precursor to the onset of OA. Such lesions often lead to surgical removal of the torn portion of the meniscus, increasing cartilage to cartilage contact area. Partial meniscectomies have been shown using finite element analysis and histology to lead to altered and increased mechanical loading on the remaining meniscus and underlying articular cartilage. Consequently, pathological compressive strains of more than $15 \%$ have been shown to increase proteoglycan breakdown and meniscal matrix degradation. Additionally, gene expression measurements merely suggest possible matrix remodeling mechanisms and do not necessarily result in protein syntheses from which matrix changes occur. Investigations in aggrecanase expression, as well as protein synthesis of key inflammatory markers, need to be performed. It is hypothesized that the catabolic response of meniscal explants will be influenced by a single bout of dynamic compression, and that disuse and overuse will result in an upregulation of aggrecanase gene expression and IL-1 activity.

It is of particular interest to inhibit the progression of inflammatory mediators involved in the degradation the menisci and potential advancement of osteoarthritis. Although many clinicians rely on steroidal and non-steroidal anti-inflammatory drugs to relieve pain and discomfort of the osteoarthritic knee, little evidence has been provided describing the efficacy and preventative measures of such treatments in the health of the knee ${ }^{146,168-171}$. These treatments may be too broad and non-specific, and may inhibit the catabolic/anabolic balance the menisci and cartilage. The use of specific pharmacological supplementation, such as that used for rheumatic diseases, to target specific catabolic pathways may provide a novel treatment for osteoarthritis and should be investigated. Extensively, it is hypothesized that the use of IL-1 Receptor Antagonist will inhibit 
the expression of specific catabolic genes, which will potentially inhibit the advancement of degeneration in the meniscus.

\section{Effect of Tibiofemoral Injury on the Meniscus}

Changes in glycosaminoglycan (GAG) gene expression and production in cartilage is

often seen during experimental $\mathrm{OA}^{172}$. Many studies in the literature use anterior cruciate ligament (ACL)-transection or medial partial or complete meniscectomy to replicate OA. This is troublesome for analyzing a real-life injury, as it does not incorporate other aspects of joint damage that may contribute to inflammation and cytokine recruitment, likely to influence tissue degradation. In the case of meniscectomy, it removes the influence of the meniscus altogether. Therefore, this research will investigate the in vivo changes in GAG coverage of menisci caused by traumatic joint injury with and without ACL tear. It is hypothesized that GAG coverage will be affected by traumatic injury, in both torn and intact ACL models. Additionally, it is hypothesized that a traumatic injury to the knee resulting in ACL rupture will demonstrate a different response of the menisci 12-weeks following injury compared to the "gold standard" ACLT model.

Acute cellular changes to the menisci following ACL rupture and meniscal tearing may also play a key role in the deterioration and degradation of the tissue. Although in vitro studies have investigated the response of both cartilage and menisci following traumatic impaction, the in vivo response to traumatic impaction on cell viability has only been minimally investigated. Therefore, it is hypothesized that traumatic impaction and ACL rupture, leading to meniscal tears, will result in an increase in cell death in both medial and lateral menisci.

\section{Research Aims}

Aim 1: To quantify the expression of aggrecanase-1 and -2 and the production of IL-1 $\alpha$ following various levels of mechanical loading. Meniscal explants were dynamically compressed for 2 hours at 0, 10 and 20\% dynamic strain. Biochemical changes were 
quantified using molecular biology techniques to determine endogenous ADAMTS expression and IL-1 $\alpha$ activity of the tissue.

Aim 2: To determine the influence of IL-1RA treatment on the matrix degradation markers following various levels of mechanical loading. Meniscal explants were dynamically compressed for 2 hours at 0, 10 and 20\% dynamic strain following treatment with and without IL-1RA. Biochemical changes were quantified using molecular biology techniques to determine anabolic and catabolic activity of the tissue.

Aim 3: To determine spatial sulfated glycosaminoglycan coverage in healthy rabbit meniscus. Menisci were stained using histological methods to determine glycosaminoglycan content distribution, cellular density, and tissue area for anterior, central, and posterior regions of medial and lateral menisci of healthy, uninjured rabbits.

Aim 4: To characterize the role of traumatic impaction on the health and degradation of the menisci. Rabbits were anaesthetized and injured using a novel closed-joint impaction model for investigating osteoarthritis in vivo. Acute and chronic changes in menisci were characterized and compared with age-matched ACLT and healthy rabbits.

Aim 5: To quantify spatial sulfated glycosaminoglycan coverage in the injured meniscus. Rabbit menisci from knees subjected to tibiofemoral impaction or ACLT were stained using histological and immunohistochemical methods to determine glycosaminoglycan coverage across all regions of both medial and lateral menisci 3 months after injury and compared with age-matched healthy rabbits.

Aim 6: To quantify the viability of meniscal cells and the production of NO following a single impaction and traumatic ACL rupture in the closed-joint lapine knee. Cell viability was determined using calcein AM and ethidium homodimer and NO was assayed using a commercially available kit. 


\section{Chapter 2 - Catabolic Response of Meniscal Tissue Explants to Dynamic Compressive Strain ${ }^{1}$}

\section{Abstract}

Objective: Following partial meniscectomy, the remaining meniscus is exposed to an altered loading environment. $20 \%$ dynamic compressive strain in vitro on meniscal tissue explants has been shown to lead to an increase in release of glycosaminoglycans from the tissue and increased expression of interleukin-1 $\alpha$ (IL-1 $\alpha$ ). The goal of this study was to determine if compressive loading which induces endogenously expressed IL-1 results in downstream changes in gene expression of catabolic molecules in meniscal tissue, such as aggrecanase expression and IL-1 $\alpha$ protein production.

Methods: Porcine meniscal explants were dynamically compressed for $2 \mathrm{~h}$ at $1 \mathrm{~Hz}$. Relative changes in gene expression of A Disintegrin and Metalloproteinase with ThromboSpondin 4 (ADAMTS4), ADAMTS5 and subsequent production of IL-1 $\alpha$ by meniscal tissue in response to varying levels of dynamic compression $(0 \%, 10 \%$, and $20 \%$ ) were measured.

Results: 20\% dynamic compressive strain upregulated ADAMTS4 compared to no dynamic loading. ADAMTS5 gene expression was upregulated under $10 \%$ strain compared to no dynamic loading. An increase in IL-1 $\alpha$ protein production was measured following $20 \%$ dynamic strain compared to $0 \%$ and $10 \%$ dynamic strain.

Conclusion: This data suggests that changes in mechanical loading of the knee joint meniscus from $10 \%$ to $20 \%$ dynamic strain can increase the catabolic activity of the meniscus.

\section{Introduction}

The menisci play a major role in load distribution and transmission in the knee joint ${ }^{30,85}$, and have been shown to be mechanically sensitive ${ }^{11,173}$. Recently, 20\% dynamic compressive strain on meniscal explants has been shown to lead to increased

\footnotetext{
${ }^{1}$ The material contained in this chapter was in part previously published the journal Osteoarthritis and Cartilage. Reprinted with permission.
} 
glycosaminoglycan (GAG) content in the culture media, an upregulation of interleukin-1 (IL-1) gene expression and increased release of nitric oxide (NO) ${ }^{32,174}$. IL-1 is a proinflammatory cytokine involved in the etiology of $\mathrm{OA}^{175,176}$. It has been shown to increase levels of $\mathrm{NO}^{176}$ which in turn can induce gene expression of other catabolic molecules such as metalloproteinases (MMPs matrix cleavage proteins) and inhibit cell proliferation $^{177-179}$. IL-1 has been shown to increase cyclooxygenase-2 (COX-2) synthesis leading to increased production of prostaglandin E2 (PGE2) in osteoarthritic cartilage ${ }^{175}$.

Certain matrix metalloproteinases (MMP-1, MMP-3, MMP-9, and MMP-13) and aggrecanases-1 and -2 (ADAMTS4 and ADAMTS5) have been shown to be responsible for the breakdown of collagens and proteoglycans in soft tissues ${ }^{105,180-184}$. Although in vitro experiments using meniscal explants have demonstrated that the menisci respond to dynamic loading by expressing elevated levels of IL-1 in a strain dependent manner ${ }^{32}$, the potential relationship of endogenous IL-1 with other catabolic activity in menisci has not been investigated. Briefly, MMP-1 and MMP-13 represent collagenases, and are involved in degradation of native collagen fibers ${ }^{130}$. MMP-3 is a representative of the stromelysin group and is partially responsible for degradation of proteoglycans and type IX collagen. The last group of MMPs (gelatinases) includes MMP-9. Gelatinases degrade denatured collagen, proteoglycan and fibronectin ${ }^{130}$. MMP-1 and MMP-13 are partially responsible for the breakdown of the helical region of fibrillar collagens ${ }^{185,}{ }^{186}$ and cleavage of the triple helices ${ }^{185}$, respectively. Tissue inhibitors of metalloproteinases (TIMPs) are elevated in OA synovial fluid and may indicate a natural attempt by the body to counteract the action of MMPs ${ }^{187}$. In a rat iodoacetate model of OA, both A Disintegrin and Metalloproteinase with ThromboSpondin (ADAMTS) and MMP neoepitopes were present, suggesting that specific cleavage of aggrecan by ADAMTS and MMP may be responsible for the degradation of aggrecan ${ }^{182}$. Over-expression of these aggrecanases results in cartilage matrix degradation ${ }^{188-190}$, and inhibition of these enzymes can prevent aggrecan degradation in vitro ${ }^{191}$. 
In articular cartilage, both cytokines and growth factors have a role in tissue homeostasis. Both IL-1 and tumor necrosis factor $\alpha(\mathrm{TNF} \alpha)$ appear to be responsible for pathological

processes in articular cartilage ${ }^{187,192,193}$. While previous studies have added exogenous IL-1 to meniscal tissue and noted degradative downstream effects ${ }^{117,136}$, it is unclear if compressive mechanical loading with endogenously expressed IL-1 results in changes of anabolic and catabolic genes in meniscal tissue. Recently, it has been shown that mechanical loading does not alter TNF $\alpha$ gene expression in meniscal tissue ${ }^{135}$. Furthermore, growth factors such as transforming growth factor $\beta$ (TGF $\beta$ ) have an important role in articular cartilage biosynthesis ${ }^{187}$, but mechanical loading of meniscal tissue alters expression of such genes has not been illustrated ${ }^{135}$.

This in vitro study was designed to measure gene expression of catabolic molecules (ADAMTS) and production of pro- and mature IL-1 $\alpha$ protein following various levels of dynamic mechanical compression of meniscal tissue.

\section{Materials and Methods}

\section{Mechanical Stimulation}

Mechanical stimulations were performed according to the previously introduced protoco $^{32}$. Briefly, six explants (5 mm tall and $6 \mathrm{~mm}$ diameter) were cut from each of 12 porcine menisci ( 6 animals, age 18 weeks). Each explant was cut perpendicular to the femoral surface of the meniscus to preserve as much superficial surface as possible and was primarily taken from the outer zone. Explants were approximately 7-8 $\mathrm{mm}$ tall at harvest and were placed in a custom device in order to cut the explants to $5 \mathrm{~mm}$ tall while preserving the superficial surface. Each explant was incubated separately for $48 \mathrm{~h}$ in growth media (89\% Dulbecco's Modified Eagle Medium/Ham's F12, 10\% Fetal Bovine Serum (FBS), $1 \%$ Penicillin Streptomycin) at $37^{\circ} \mathrm{C}$ with $5 \% \mathrm{CO}_{2}$ to equilibrate. Media were changed every $24 \mathrm{~h}$. A total of 72 explants were harvested and 54 were randomly used for the study. Nine explants from each animal were randomly selected and exposed to 1 of 3 loading protocols $(0 \%, 10 \%$ or $20 \%$ dynamic compression strain, with three explants per loading protocol). Mechanical stimulation was performed with a custom 
built bioreactor $^{32,174}$. All explants were unconstrained for $48 \mathrm{~h}$ during which time no visual swelling was noted. Therefore, all strain measurements assumed no swelling of the tissue. Following this period, a preload of $0.0076 \mathrm{MPa}$ was applied by the platen, and six explants were simultaneously loaded to either $10 \%$ or $20 \%$ dynamic compressive strain or $0 \%$ compressive loading (tare weight of the platen remained as a static load) for $2 \mathrm{~h}$ at $1 \mathrm{~Hz}$ to simulate walking. It has previously been shown that when using this bioreactor, $10 \%$ dynamic strain results in a peak stress of $1.41 \pm 0.10 \mathrm{MPa}$ and an equilibrium stress of $0.046 \pm 0.01 \mathrm{MPa}$, while $20 \%$ dynamic strain results in peak and equilibrium stresses of $3.55 \pm 0.43 \mathrm{MPa}$ and $0.13 \pm 0.02 \mathrm{MPa}$, respectively ${ }^{174}$. The equilibrium stress was indicated as the measured value after $2 \mathrm{~h}$ of dynamic loading, at which the stress did not significantly change with each cycle. Following mechanical stimulation, explants were first cut in half to separate superficial and deep zones tissue and then weighed and placed in media for $24 \mathrm{~h}$ at $37^{\circ} \mathrm{C}$ with $5 \% \mathrm{CO}_{2}$. The explants were then treated with RNALater (Ambion Inc., Austin, TX) for $24 \mathrm{~h}$ prior to storage. The superficial explants represent the top $2.5 \mathrm{~mm}$ of the meniscus that contacts the femur while the deep explants represent the middle $2.5 \mathrm{~mm}$ of the tissue.

\section{Gene Expression}

To isolate an adequate amount of RNA for gene analysis three explants from a given animal that received the same loading treatment were combined for total RNA isolation using a commercially available kit (SV Total RNA Isolation System, Promega, Madison, WI) as previously described ${ }^{32}$. The RNA quality was verified by running 200ng of RNA on a $1.5-2.5 \%$ ethidium bromide-stained agarose gel, and visualizing the intact large and small ribosomal subunits under ultraviolet light. Reverse Transcription (RT) and realtime Polymerize Chain Reaction (PCR) were carried out to measure gene expression in mechanically stimulated meniscal tissue as well as calibration (control) tissue.

RT reactions began with a $12 \mathrm{~mL}$ reaction, consisting of $300 \mathrm{ng}$ RNA, 100ng random primers and $0.83 \mathrm{mM}$ dNTPs. Samples were heated to $65^{\circ} \mathrm{C}$ for $5 \mathrm{~min}$ and then placed on ice. At this time, a $7 \mathrm{~mL}$ mix consisting of $4 \mathrm{~mL}$ of the $5 \mathrm{x}$ buffer provided with the 
SuperScript II (Invitrogen Corporation, Carlsbad, CA, USA), 0.029 M dithiothreitol (DTT) and 40 units of RNase Out (Invitrogen Corporation, Carlsbad, CA, USA) were added to each reaction. The reaction was incubated at $25^{\circ} \mathrm{C}$ for $2 \mathrm{~min}$ and again placed on ice while 50 units of SuperScript II (Invitrogen Corporation, Carlsbad, CA, USA) were added to each reaction. Tubes were then incubated at $25^{\circ} \mathrm{C}$ for $10 \mathrm{~min}$, followed by $42^{\circ} \mathrm{C}$ for $50 \mathrm{~min}, 70^{\circ} \mathrm{C}$ for $15 \mathrm{~min}$ and $4^{\circ} \mathrm{C}$ for $5 \mathrm{~min}$. Samples were placed on ice, and 2 units RNase H (New England BioLabs, Ipswich, MA, USA) were added, bringing the final reaction volume to $20 \mathrm{~mL}$. Samples were incubated at $37^{\circ} \mathrm{C}$ for $20 \mathrm{~min}$, followed by 20 min at $65^{\circ} \mathrm{C}$, and $5 \mathrm{~min}$ at $4^{\circ} \mathrm{C}$. All reactions were performed in an Eppendorf Mastercycler Gradient machine (Westbury, NY, USA).

Real-time PCR was performed in $25 \mathrm{~mL}$ reaction volumes using gene specific primers designed from partial or complete Sus scrofa cDNA sequences available from National Center for Biotechnology Information (NCBI), or by using primers from the literature ${ }^{194}$ (Table 2-1). Newly designed primers were created using the PrimerQuest and OligoAnalyzer 3.1 software (Integrated DNA Technologies, Coralville, IA, USA). The ribosomal 18s RNA was used as a housekeeping gene and was run on each plate alongside the gene of interest. Reactions for genes contained 15ng of cDNA (ADAMTS4, ADAMTS5 and 18s [when used a as a housekeeper for these genes]) with $0.1 \mathrm{mM}$ of each primer, 12.5mL SYBR Green Fluorescence Mix. All qPCR reactions were run on a Stratagene MX3000P QPCR System (La Jolla, CA, USA), and began with an initial denaturation of $15 \mathrm{~min}$ at $95^{\circ} \mathrm{C}$. This was followed by 40 cycles of: $95^{\circ} \mathrm{C}$ for $15 \mathrm{~s}, 60 \mathrm{~s}$ at $55^{\circ} \mathrm{C}$ and $40 \mathrm{~s}$ at $72^{\circ} \mathrm{C}$. This was followed by a dissociation curve analysis to verify the specificity of the amplification. All samples were run in duplicate and data were analyzed using Stratagene MXPro QPCR Software (La Jolla, CA, USA). A dissociative curve was run with each plate setup to confirm regularity of the tests. Samples were analyzed using the Pfaffl method by which the ratio of the target gene to the housekeeping gene is quantified with respect to the calibrator using the following formula: 


$$
\text { ratio }=\frac{\left(E_{\text {target }}\right)^{\Delta C_{t} \text { target(control-treated) }}}{\left(E_{\text {ref }}\right)^{\left.\Delta C_{t} \text { ref(control-treated }\right)}}
$$

where $\mathrm{Ct}=$ cycle number in the linear range of amplification, $\mathrm{E}=$ efficiency of the process for each gene, target $=$ gene of interest, ref $=$ housekeeping gene, control = calibrator, treated $=$ sample.

Table 2-1. Primer sequences used for specific genes.

$\begin{array}{lllcc}\text { Gene } & \text { Sequences } & \text { Denaturation } & \text { Reference } \\ \text { ADAMTS4 } & 5^{\prime} & \text { AGGAGGAGATCGTGTTTCCAGAGA } & 55^{\circ} \mathrm{C} & 135 \\ & 3^{\prime} & \text { AAAGGCTGGCAAGCGGTACAACAA } & & \\ \text { ADAMTS5 } & 5^{\prime} & \text { TTCGACATCAAGCCATGGCAACTG } & 55^{\circ} \mathrm{C} & 135 \\ & 3^{\prime} & \text { AAGATTTACCATTAGCCGGGCAGG } & & \\ 18 s & 5^{\prime} & \text { GCAAATTACCCACTCCCGAC } & 55^{\circ} \mathrm{C} & 32,135 \\ & 3^{\prime} & \text { CGCTCCCAAGATCCAACTAC } & & \end{array}$

\section{Western Blotting}

Protein was isolated from superior and deep sections of the $0 \%, 10 \%$ and $20 \%$ dynamic strain tested explants. Three frozen explants $(5 \mathrm{~mm}$ diameter; $2.5 \mathrm{~mm}$ thick) from each respective testing group/location were used for one protein extract sample and were pulverized in liquid nitrogen using mortar and pestle to make one sample. The pulverization set-up was wiped with $70 \%$ isopropanol and PBS between samples. The samples were weighed using microcentrifuge tubes and kept on ice. Radioimmunoprecipitation assay buffer (RIPA, 1\% Igepal, 0.5\% sodium deoxycholate, $0.1 \%$ SDS, in PBS) was added to each sample (3ml RIPA/1g tissue) and mixtures were vortexed for 60s and placed on ice for $45 \mathrm{~min}$. Each sample was then homogenized using a polytron twice for $15 \mathrm{~s}$. Upon homogenization, the samples were centrifuged at $14,000 \mathrm{~g}$ for $10 \mathrm{~min}$ at $4^{\circ} \mathrm{C}$. The supernatant was extracted and stored at $-80^{\circ} \mathrm{C}$ and the pellet was discarded. After proper homogenization of the samples, supernatant were collected and protein was quantified using the modified Lowry method. 
Total protein isolates of independent samples ( $10 \mu \mathrm{g}$ total protein each) were separated on 16\% SDS-PAGE gels (PAGEgel, Inc., San Diego, California, USA). PAGEgel 2-Color SDS Marker was used as a molecular weight standard. Transfer of protein onto nitrocellulose membranes ( $0.2 \mu \mathrm{m}$ pore size; Pierce Biotechnology, Rockford, IL) was performed at $50 \mathrm{~V} / 250 \mathrm{~mA}$ using tris-glycine-SDS transfer buffer with $10 \%$ methanol at $4^{\circ} \mathrm{C}$. Membranes were removed and rinsed in PBS, and blocked in Odyssey blocking buffer (LI-COR, Lincoln, NE) overnight at $4^{\circ} \mathrm{C}$. To determine the effects of altered mechanical loading on catabolic molecular production, the membrane was then incubated with primary antibody solution $(0.1 \mathrm{ug} / \mathrm{ml}$ biotinylated anti-porcine IL- $1 \alpha$ antibody in Odyssey blocking buffer; R\&D Systems, Minneapolis, MN) overnight at $4^{\circ} \mathrm{C}$ with constant shaking. Blots were then conjugated with secondary antibody solution $(0.2 \mathrm{ug} / \mathrm{ml}$ biotinylated goat anti-mouse IgG in Odyssey blocking buffer; Upstate, Billerica, MA) and fluorescently labeled with Alexa Fluor 680 Streptavidin $(0.4 \mathrm{ug} / \mathrm{ml}$, Invitrogen, Carlsbad, CA). Following this, the membrane was washed, rinsed and fluorescently visualized with the LI-COR Odyssey Infrared Imaging System (LI-COR Biosciences, Lincoln, NE). Recombinant porcine interleukin-1 $\alpha$ (R\&D Systems, Minneapolis, MN) of varying dilutions was used as a positive control.

\section{Data Analysis}

All data are presented as mean \pm standard deviation (SD). A repeated measures ANOVA with Fisher's protected least significant difference method was used to calculate statistical differences $(\mathrm{P}<0.05$ was considered significant) between different loading conditions for both zones. Paired t-tests were used to measure differences in cell response of pair-wise superficial and deep zones for each loading conditions $(\mathrm{P}<0.05$ was considered significant).

For western blotting, semi-quantitative comparisons between band intensities of samples were performed using a custom MATLAB program (MathWorks, Natick, MA). 


\section{Results}

The expression of two ADAMTS genes following various levels of mechanical compression was studied (Figure 2-1). There was a significant upregulation of ADAMTS5 with $10 \%$ dynamic compressive strain compared to no and $20 \%$ dynamic strain tests for deep explants. On the other hand, 20\% strain upregulated ADAMTS4 expression for superficial explants compared to $10 \%$ strain. ADAMTS4 was significantly upregulated at no dynamic strain compared to $10 \%$ dynamic strain tests for superficial explants.

Western blot techniques were implemented to determine if changes in IL-1 $\alpha$ protein production are also evident following pathological loading (Figure 2-2). Results show that samples tested at physiological levels of compressive strain (10\%) demonstrate minimal expression of a pro-IL-1 $\alpha$ aggregate.

\section{Discussion}

This study was designed to investigate the response of meniscal explants to various magnitudes of dynamic compressive strain while measuring changes in gene expression of catabolic molecules and the production of IL-1 $\alpha$. The results suggest that $2 \mathrm{~h}$ of dynamic loading at $20 \%$ strain increase expression of catabolic enzymes involved in the degradation of proteoglycans, namely aggrecanases (ADAMTS4), whereas 10\% dynamic strain increases ADAMTS5 compared to no dynamic loading.

Previous computational studies by our group suggest that removing $30 \%$ or more or the meniscus increases the maximum compressive strains in the remaining meniscal tissue to approximately $20 \%{ }^{95}$, compared to $10 \%$ strain in the intact meniscus. Hence, dynamic strain values of $10 \%$ and $20 \%$ were compared in this study. It was expected that both under-loading ( $0 \%$ dynamic strain) and overloading (20\%) would increase catabolic gene expression compared to $10 \%$ dynamic strain. Indeed, overloading and no loading resulted in increased gene expression for ADAMTS4 compared to $10 \%$ dynamic strain. Of genes explored in this and of previous studies ${ }^{32}$, ADAMTS4 was the only catabolic gene 
upregulated following under-loading compared to $10 \%$ strain, which may indicate that the period of no dynamic loading in this study ( 2 days) is insufficient to induce a complete degradative pathway involving MMPs. Previous studies have shown that exposing meniscal explants to cyclic hydrostatic pressure for $4 \mathrm{~h}$ prevented upregulation of COX-2, IL-1, MMP-1, MMP-3, and iNOS that was seen in unloaded explants ${ }^{195}$. Djurasovic et al. showed that 4 weeks of immobilization in a canine model resulted in a decreased aggrecan gene expression in the menisci ${ }^{68}$. Further long-term studies are needed to investigate if long-term lack of dynamic loading adversely affects meniscal tissue composition and potential mechanisms, as the current study only considered gene expression following $24 \mathrm{~h}$ of post-incubation.

The down-regulation of ADAMTS5 following no dynamic compressive strain compared to $10 \%$ strain is worth noting, as it was expected that catabolic expression would be influenced by pathological, not physiological, dynamic strain. As this study is a single time-point of $2 \mathrm{~h}$ of dynamic loading with $24 \mathrm{~h}$ post-incubation, it will be important to investigate the expression of such molecules over longer periods of time in order to better understand the temporal expression patterns following mechanical loading.

In the present study, all genes and protein production were measured at the same postincubation time point based on previous studies ${ }^{174}$. Earlier observations of cytokine and anabolic gene expression profiles following mechanical loading demonstrated a maximum expression of ADAMTS4, MMP-3 and MMP-9, as well as COL-1 after $24 \mathrm{~h}$ of mechanical loading ${ }^{179,}{ }^{196}$. Nonetheless, changes in the expression of the genes of interest may be influenced by both the in vitro approach and/or the choice in postincubation time period.

Protein analysis performed in this study demonstrated the presence of a pro-IL-1 $\alpha$ aggregate around $70 \mathrm{kDa}$. This aggregation is approximately twice the molecular weight of the native pro-IL-1 $\alpha$ isoform. Although it is possible that the monoclonal anti-porcine IL-1 $\alpha$ antibody used in these experiments cross-reacted with a larger, non-specific 
protein, it is not likely. The use of monoclonal antibodies provides greater specificity than polyclonal antibodies, and such specific antibodies can help determine changes in molecular conformations, phosphorylation states of proteins, and protein-protein interactions ${ }^{197}$. Additionally, validation of the blotting and antibody specificity was performed using recombinant porcine IL-1 $\alpha$ protein run simultaneously on the same gel as the experimental samples, which showed distinct bands at the appropriate molecular weight for IL-1 $\alpha(17 \mathrm{kDa})$. It is possible that interaction between glycosylation and/or aggregations of pro-IL- $1 \alpha$ protein, or the presence of pro-IL-1 $\alpha$ dimers, may exist if proteins were not fully reduced or denatured during the preparatory process. Dimeric forms have been observed following blotting of IFN- $\beta^{198}$. Use of endoglycosidases, such as Peptide: N-Glycosidase F (PNGase-F), may help prevent dimers in future tests.

Investigations in cytokine gene expression following differential loading may provide insight into the etiology of osteoarthritis. The work by Gupta et al. suggesting the upregulation of IL-1 $\alpha$ gene expression caused by abnormal compressive strains was supported by recent immunoblotting for IL- $1 \alpha^{32}$. The presence of IL-1 $\alpha$ protein encourages apoptosis and matrix degradation by inhibiting repair, which may contribute to the degeneration of the meniscus, and ultimately lead to knee joint osteoarthritis. Further investigations of aggrecanase gene expression were also performed, suggesting mechanical influence on cytokine transcription ${ }^{135}$. Compressive strains of $20 \%$ have been shown to result from removing $30-60 \%$ of the meniscus during partial meniscectomy ${ }^{95}$. These data, taken together with current data showing increased pro-IL-1 $\alpha$ production following $20 \%$ dynamic compressive strains and increased aggrecanase gene expression, suggests that removing $30 \%$ or more of the meniscus may results in matrix disruption mediated by IL- $1 \alpha$.

The inner region of the meniscus is typically removed during partial meniscectomy, and therefore only outer meniscal explants were investigated in the present study. Meniscal tissue is comprised of at least three relatively distinct cell populations ${ }^{199}$ and cells from different regions of the meniscus have been shown to exhibit varying cell morphology 
and gene expression ${ }^{57,200,201}$. As in previous experiments from our lab, there were limited statistical differences between superior and deep explants ${ }^{32,}{ }^{174}$, possibly due to an inefficient separation of the different cell populations. Previous investigations have demonstrated differences between superficial and deep zones of the tissue following mechanical stimulation ${ }^{56}$. Future studies should include collection and compression of explants from both inner and outer regions, as well as immunostaining of explants to determine spatial expression and production of genes and molecules to account for potential ineffectiveness of isolation between different cellular populations.

Bisection of the explants into superior and deep zone has previously been shown to release many growth factors which may present misleading expression results ${ }^{202}$. However, all samples in this study were bisected, and therefore the relative differences are still significant. It is possible that the measured response may be due to both mechanical stimuli and cutting the samples, and absolute values should be interpreted with caution. The potential for complete unloading of the explants may arise due to platen lift-off during dynamic compression. In this study, we measured loading stresses during compression and recovery of each cycle during the $2 \mathrm{~h}$ period. However, it is possible that platen lift-off from the explants occurred, especially during several of the initial cycles for $20 \%$ strain. Regardless, measured stress at or above the measured tareload returned following roughly 5-10 cycles (data not shown). The recovery of the explants to original height during dynamic compression may vary depending on the native anatomical location of the explant. For example, in vivo MRI imaging of meniscal thickness reflects differences in rate of recovery following distance running in humans ${ }^{112}$. Variation in thickness recovery, and therefore duration of platen lift-off, may depend on rate of compression as well as whether the explant is of lateral or medial meniscal origin.

A finite element model of the knee has demonstrated that $20 \%$ axial strains may be indicative of strain levels seen following removal of $30 \%$ or more of the meniscus ${ }^{95}$. However, it is important to note this model used an intact meniscus, not an isolated meniscal explants, which may lead to different stresses due to lack of confinement of the 
surrounding tissue. The current study shows that strains of $20 \%$ initiate expression of several catabolic enzymes that have been previously found to degrade proteoglycans in the solid matrix of meniscal tissue ${ }^{175,189,203}$. Thus, the remaining meniscal tissue may be vulnerable to degeneration following certain partial meniscectomies. Future in vivo studies should investigate changes in morphology, gene expression, and matrix molecules of the remaining meniscal tissue following partial meniscectomy. It is unknown if the gene expression found in this study would translate to protein expression and eventually a change in the material properties of the remaining meniscal tissue. This would likely further exacerbate the degeneration of the underlying articular cartilage.

This data, in conjunction with our previous studies, suggests that dynamic loading of $20 \%$ increases ADAMTS-4 gene expression which could lead to meniscal tissue degeneration. These data are some of the first to suggest that the remaining meniscus following partial meniscectomy may contribute to the pathology of the knee joint beyond just alteration in loads on the underlying articular cartilage. Matrix degradation of the remaining meniscus may alter the material properties of the meniscus tissue rendering it unable to protect the underlying cartilage. It remains to be determined whether mechanically induced IL-1 in the meniscus is responsible for changes in gene expression.

\section{Recommendations}

It is important to explore the role of IL-1 in the biochemical response of meniscal tissue.

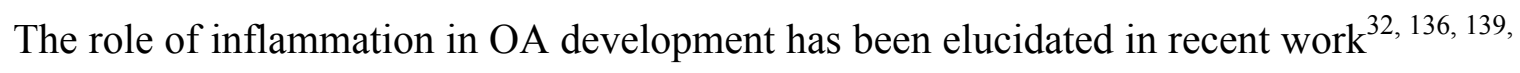
192. However, its impact on meniscal integrity and degradation is not well understood. Therefore, future studies blocking the IL-1 pathway may help to identify specific avenues of mechanotransduction that lead to matrix degradation and, ultimately, OA. 

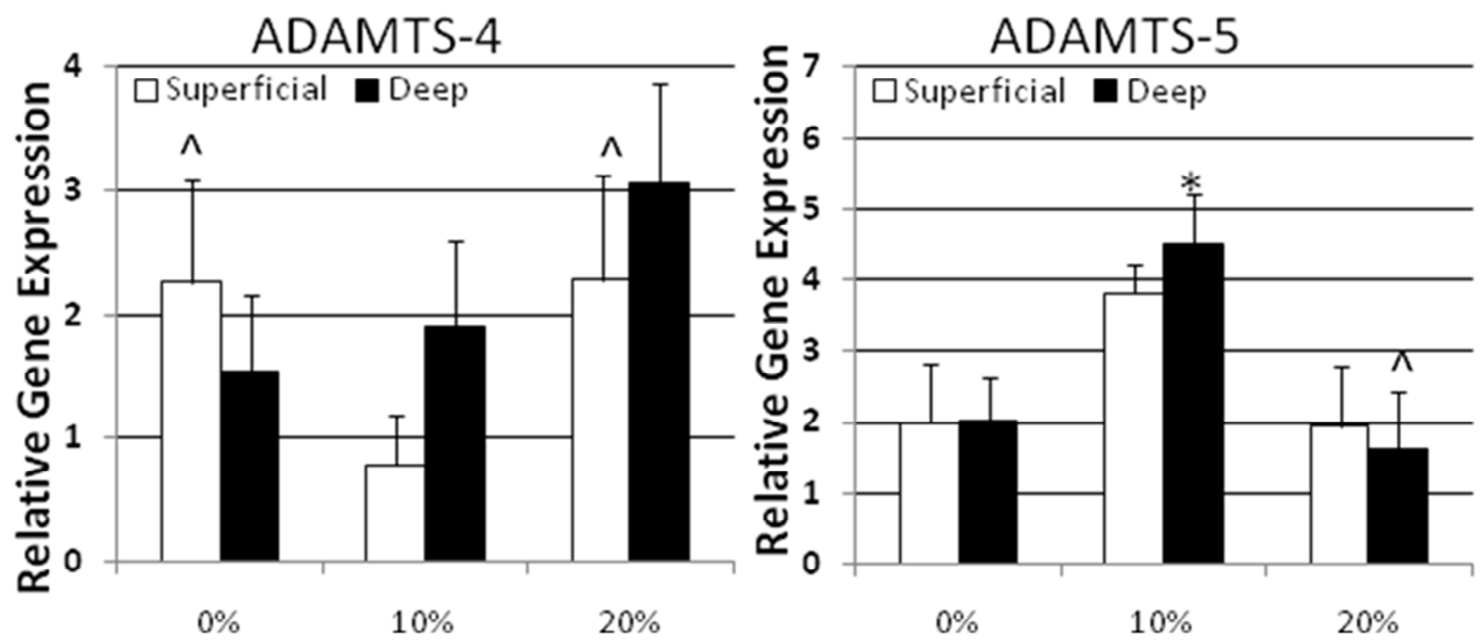

Figure 2-1. Gene expression of ADAMTS-4 and -5 following $0 \%, 10 \%$ or $20 \%$ dynamic compressive strain administered for $2 \mathrm{~h}$ at $1 \mathrm{~Hz}$ relative to calibrator gene expression. Expression normalized to 18srRNA gene expression for each sample. * Significantly different than $0 \%$ for the same zone; ${ }^{\wedge}$ significantly different than $10 \%$ for the same zone.

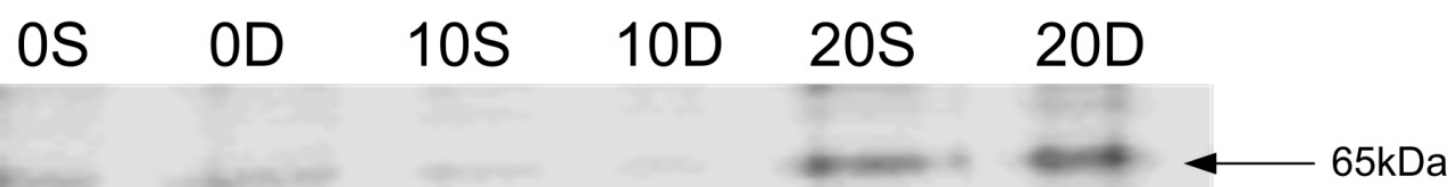

Figure 2-2. Immunoblot of endogenously produced protein in superficial (S) and deep (D) explants compressed to 0,10 , or $20 \%$ strain levels. 


\section{Chapter 3 - In vitro Inhibition of Compression-Induced Catabolic Gene Expression in Meniscal Explants Following Treatment with IL-1 Receptor Antagonist ${ }^{2}$}

\section{Abstract}

Background: Damage to the knee meniscus may result in tears that are difficult or unable to heal, and are often treated by partial removal of the damaged tissue. In vitro $20 \%$ dynamic compressive strains on meniscal tissue explants have shown an increase in release of sulfated glycosaminoglycans (GAG) and nitric oxide (NO) from the tissue explants and increased expression of matrix metalloproteinases and interleukin-1 $\alpha$ (IL$1 \alpha$ ). The objective of this study was to explore the efficacy of IL-1 blockade on the expression of a wide range of genes, as well as NO and GAG release, following dynamic compression of porcine meniscal explants.

Methods: Explants were dynamically compressed for $2 \mathrm{hrs}$ at $1 \mathrm{~Hz}$ to $0 \%, 10 \%$, or $20 \%$ strain with and without a pre-treatment of $500 \mathrm{ng} / \mathrm{ml}$ IL-1RA. Relative changes in gene expression of IL-1 $\alpha$, MMP-1, -3, -13, A Disintegrin and Metalloproteinase with ThromboSpondin 4 (ADAMTS-4), ADAMTS-5, iNOS, Aggrecan, and COX-2, as well as changes in NO and GAG release, were measured with standard biochemical assays.

Results: Expression of IL-1 $\alpha$, MMP-3, MMP-13, and ADAMTS-4 in superficial explants was significantly down-regulated at $20 \%$ dynamic strain compared to $10 \%$ strain following treatment with IL-1RA. GAG and NO release were not significantly influenced by IL-1RA treatment.

Conclusions: Treatment of meniscal explants with IL-1RA inhibited the expression of many catabolic genes following a single bout of high dynamic strain. IL-1RA may therefore be a potential therapy option during the acute phase of meniscal tear or meniscectomy treatment.

${ }^{2}$ The material contained in this chapter was in part previously published the journal Journal of Orthopaedic Science. Reprinted with permission. 


\section{Introduction}

Torn menisci are often treated by removal of the damaged portion of tissue, known as a partial meniscectomy, which has been shown to lead to changes in the stress and strain distribution of the remaining menisci ${ }^{95}$. Loss of meniscal function results in the inevitable onset of $\mathrm{OA}^{204}$. Understanding the load-dependent metabolism of meniscal tissue is important for the development of clinically relevant repair and rehabilitation methods including tissue-engineered meniscal replacements.

An imbalance of pro- and anti-inflammatory cytokines is understood to play a role in the progression of $\mathrm{OA}^{130}$. This imbalance can ensue following altered mechanical loading, a process also known as mechanotransduction. Previous studies have illustrated an

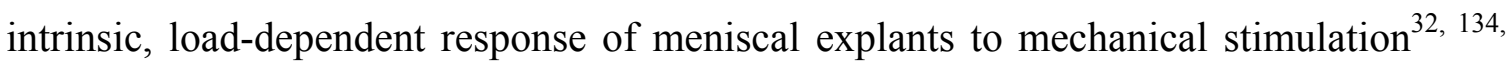
135, 174. Such catabolism caused by altered loading may contribute to the degradation of the meniscal matrix following altered loading, and result in a reduced affinity for the meniscus to perform its role in load distribution and joint lubrication. The proinflammatory Interleukin-1 (IL-1) protein discourages meniscal biosynthesis and repair ${ }^{99}$, and its pathway may be activated by excessive dynamic mechanical stimulation ${ }^{32}$. Both $\alpha$ and $\beta$ isoforms of IL-1 protein bind to the type 1 IL-1 receptor protein (IL-1R1) on the cell membrane ${ }^{205}$. This binding activates the IL-1 Receptor Associated Kinases (IRAK-1 and IRAK-2) ${ }^{205}$ which then upregulate further expression and production of IL-1 and its inflammatory sequelae ${ }^{206}$. However, binding of IL-1 isoforms to the IL-1R1 site is regulated by IL-1 receptor antagonist (IL-1RA) protein, a naturally occurring peptide that is a current therapeutic treatment for rheumatoid arthritis. IL-1RA inhibits the activity of IL-1 isoforms by competitively binding to the IL-1R 1 site $^{207}$ and thus preventing the downstream signaling cascade of proteinase activity.

Exogenous IL-1 $\alpha$ has been shown to inhibit compression-induced proteoglycan synthesis in meniscal explants ${ }^{111}$ and inhibit the healing potential of menisci following tissue culture in vitro ${ }^{136}$. However, the role of endogenously produced IL-1 in meniscal tissue is not well understood. Previous studies have discovered the endogenous expression and 
production of IL- $1 \alpha$ following a single $2 \mathrm{hr}$ bout of $20 \%$ dynamic strain ${ }^{32,134}$, as well as the load-dependency in expression of several other catabolic molecules including matrix metalloproteinases (MMP) and aggrecanases (ADAMTS) ${ }^{135}$. However, it is not yet known if the IL-1 pathway alone is responsible for intrinsic meniscal degradation in vitro. Therefore, the objective of this study was to explore the role of IL-1 blockade in the expression of a wide range of genes following dynamic compression of porcine meniscal explants. We hypothesized that the introduction of IL-1RA in the media of meniscal explants will inhibit the cascade of catabolic gene expression, the production of nitric oxide (NO), and the breakdown of proteoglycan (GAG) released to the media after a single bout of dynamic compression.

\section{Materials and Methods}

\section{Tissue preparation}

Stifle joints of 18-week old pigs were dissected using aseptic techniques to expose the menisci. Menisci were removed from the joint by excision of horn, ligamentous, and synovial attachments. Explants were extracted from the femoral surface of the outer zone of each menisci (6-mm diameter) using a biopsy punch. Explants were trimmed to $5 \mathrm{~mm}$ from the deep surface, keeping the femoral surface of the explants intact, and rinsed in sterile PBS and equilibrated in the growth media (44.5\% Dulbecco's Modified Eagle Medium/Ham's F12, 10\% Fetal Bovine Serum, 1\% Penicillin Streptomycin) for a preincubation period of $48 \mathrm{~h}$ at $37^{\circ} \mathrm{C}\left(5 \% \mathrm{CO}_{2}\right)$. During the last $24 \mathrm{~h}$ of pre-incubation, explants were assigned to one of two different main groups; control and IL-1RA treated. Briefly, explants in the control group were incubated in fresh growth media alone for this pre-incubation duration as well as during the mechanical test. Explants from the IL-1RA treated group were incubated in growth media containing $500 \mathrm{ng} / \mathrm{ml}$ of recombinant porcine IL-1RA (R\&D Systems, Inc., Minneapolis, MN, USA, \#780-RA) for the remaining pre-incubation duration as well as during the mechanical test. This dosage was determined in order to assure maximum blockage of the IL-1 pathway ${ }^{208}$. 


\section{Cyclic loading protocol}

Explants were assigned to one of three compressive loading subgroups within each treatment group. Under sterile conditions, six explants (3 from control group and 3 from IL-1RA treated group) were simultaneously loaded in individual wells in a custom built bioreactor to 0,10 or $20 \%$ dynamic compressive strain while maintained at $37^{\circ} \mathrm{C}$ in $400 \mu \mathrm{L}$ of growth media either with or without IL-1RA. All explants were dynamically loaded for $2 \mathrm{~h}$ at $1 \mathrm{~Hz}$ frequency. The bioreactor was carefully cleaned following dynamic compression exercises to avoid cross-contamination of media between groups. Following mechanical stimulation, explants were halved transversely to separate superficial and deep zones, weighed, and placed for $24 \mathrm{~h}$ in $1 \mathrm{~mL}$ of post-incubation media $(48.5 \%$ Dulbecco's Modified Eagle Medium/Ham's F12, 2\% Fetal Bovine Serum, 1\% Penicillin Streptomycin) at $37^{\circ} \mathrm{C}\left(5 \% \mathrm{CO}_{2}\right)$. At this time, no explants were stored with recombinant IL-1RA. Following post-incubation, explants were treated with RNALater (Ambion Inc., Austin, TX, USA) for $24 \mathrm{~h}$ and stored at $-20^{\circ} \mathrm{C}$.

\section{RNA extraction}

Total RNA was isolated from superior and deep explants according to the protocol previously described using a commercially available kit (SV Total RNA Isolation System, Promega, Madison, WI, USA) ${ }^{135}$. Based on previous studies, 3 explants are needed to acquire the appropriate concentration of RNA for reverse transcription ${ }^{32,135}$. Therefore, 3 simultaneously loaded explants from each treatment group were combined and crushed to a fine powder using liquid nitrogen ( $\mathrm{n} \geq 5$ for each group). The powder was immediately added to lysis buffer and stored at $4^{\circ} \mathrm{C}$. A homogenizer was used to lyse the cells further. RNA was isolated and DNase-treated using a spin basket assembly and then eluted in $100 \mu 1$ of nuclease free water. Integrity of isolated RNA was assessed by ethidium bromide staining after running samples on a $1.5 \%$ native agarose gel to check $18 \mathrm{~S}$ rRNA and $28 \mathrm{~S}$ bands. RNA samples were then stored at $-80^{\circ} \mathrm{C}$ until used for reverse transcription. At least five independent RNA isolations were performed for each mechanical strain group $(0,10$, and $20 \%)$ for each treatment group. While control explants were used in the present study, data from previous experiments involving 
meniscal explants without IL-1RA were combined if variance of previous and present groups were equivalent ${ }^{135}$.

\section{Reverse-transcription-polymerase chain reaction}

RNA quality and concentration immediately prior to reverse transcription (RT) was determined by measurement of the optical density at $260 \mathrm{~nm}$ on a NanoDrop 2000 Spectrophotometer (ThermoScientific, Wilmington, DE, USA). First-strand RT began with a $12 \mu \mathrm{L}$ reaction consisting of $300 \mathrm{ng}$ RNA, $100 \mathrm{ng}$ random primers and $0.83 \mathrm{mM}$ dNTPs. Samples were heated to $65^{\circ} \mathrm{C}$ for $5 \mathrm{~min}$ and then placed on ice. At this time, a $7 \mu \mathrm{l}$ mix consisting of $4 \mu 1$ of the 5 ' buffer provided with the Superscript II (Invitrogen Corporation, Carlsbad, CA, USA), 0.029M dithiothreitol (DTT) and 40units of RNase OUT (Invitrogen Corporation, Carslbad, CA, USA) were added to each reaction. The reactions were incubated at $25^{\circ} \mathrm{C}$ for $2 \mathrm{~min}$ and again placed on ice while 50units of Superscript II were added to each reaction. Tubes were then incubated at $25^{\circ} \mathrm{C}$ for $10 \mathrm{~min}$ followed by $42^{\circ} \mathrm{C}$ for $50 \mathrm{~min}, 70^{\circ} \mathrm{C}$ for $15 \mathrm{~min}$, and $4^{\circ} \mathrm{C}$ for $5 \mathrm{~min}$. Samples were placed on ice and treated with RNase H (New England BioLabs, Ipswich, MA, USA), bringing the final reaction to $20 \mu 1$. Samples were incubated at $37^{\circ} \mathrm{C}$ for $20 \mathrm{~min}$, followed by $29 \mathrm{~min}$ at $65^{\circ} \mathrm{C}$ and $5 \mathrm{~min}$ at $4^{\circ} \mathrm{C}$. All reactions were performed in an Eppendorf Mastercycler Gradient machine (Westbury, NY, USA).

\section{Real-time quantitative-PCR}

Real-time quantitative PCR (q-PCR) reactions were performed with SYBR Green fluorescence mix (Absolute QPCR SYBR Green Mix, Abgene, Inc, NY; Fast SYBR Green Master Mix, Applied Biosystems, Foster City, CA). Results of copy number fluorescence threshold $(\mathrm{Ct})$ of each gene were normalized to a housekeeping gene (Ribosomal 18sRNA). A calibration control sample was run on each plate and expression of the housekeeping gene for each sample was run in each reaction alongside each gene of interest. Q-PCR was performed in $25 \mu \mathrm{L}$ reaction volumes using gene specific primers designed from partial or complete Sus scrofa cDNA sequences available from National Center for Biotechnology Information (NCBI), or by using primers from the literature ${ }^{135}$ (Table 3.1). Reactions for most genes (IL-1 $\alpha$, MMP-1, -3, -13, COX-2, iNOS and 18s 
[when used a as a housekeeper for these genes]) contained 7.5ng of cDNA, $0.2 \mathrm{mM}$ of each primer, $12.5 \mathrm{~mL}$ SYBR Green Fluorescence Mix. For the remaining genes (Aggrecan, ADAMTS4, ADAMTS5), 15ng of cDNA was used in each reaction, with $0.1 \mathrm{mM}$ of each primer, $12.5 \mathrm{~mL}$ SYBR Green. All qPCR reactions were run on a StepOnePlus Real Time PCR system (Applied Biosystems, Foster City, CA), and began with an initial denaturation of $15 \mathrm{~min}$ at $95^{\circ} \mathrm{C}$. This was followed by 40 cycles of: $95^{\circ} \mathrm{C}$ for $15 \mathrm{~s}, 60 \mathrm{~s}$ at denaturation temperature (Table 3-1) and 40s at $72^{\circ}$. This was followed by a dissociation curve analysis to verify the specificity of the amplification. All samples were run in duplicate and $\mathrm{Ct}$ values were obtained using StepOne Software v2.1 (Applied Biosystems, Foster City, CA). A dissociative curve was run with each plate setup to

confirm regularity of the tests. Sample Ct were analyzed using the Pfaffl method ${ }^{209}$ by which the ratio of the target gene to the housekeeping gene is quantified with respect to the calibrator using Equation 2-1.

\section{Statistical analysis}

All data are presented as mean \pm standard error. Statistical analyses comparing levels of assay results (gene expression) between control and IL-1RA treated samples was performed using a two-way analysis of variance (2-way ANOVA) with treatment and strain level assigned as independent variables. A post-hoc Fisher's protected least squares difference was carried out. $\mathrm{P}<0.05$ was considered significant. 
Table 3-1. Primer sequences used for specific genes.

\begin{tabular}{|c|c|c|c|}
\hline Gene & Sequences & Denaturation & Reference \\
\hline \multirow[t]{2}{*}{$I L-1 \alpha$} & 5' AGACACCCAAAACCATCAAAG & $59^{\circ} \mathrm{C}$ & 32 \\
\hline & 3' TCACAGGTAAGTAGACACCAG & & \\
\hline \multirow[t]{2}{*}{ iNOS } & 5' ACGCTCAGCTCATCCGGTAT & $61^{\circ} \mathrm{C}$ & 32 \\
\hline & 3'CACTTCAGCTCCAGCTCCTG & & \\
\hline \multirow[t]{2}{*}{$M M P-1$} & 5'GGACCTGGAGGAAACCTTGCT & $59^{\circ} \mathrm{C}$ & 135 \\
\hline & 3' GCCTGGATGCCATCAATGTC & & \\
\hline \multirow[t]{2}{*}{$M M P-3$} & 5' GCCTGGATGCCATCAATGTC & $59^{\circ} \mathrm{C}$ & 135 \\
\hline & 3' TCTTGGAGAATGTAAGCGGAGT & & \\
\hline \multirow[t]{2}{*}{$M M P-13$} & 5'GATCCCCATTTTGATGATGATGAA & $59^{\circ} \mathrm{C}$ & 135 \\
\hline & 3'GTCTTCATCTCCTGGACCATAGAGAGA & & \\
\hline \multirow[t]{2}{*}{$C O X-2$} & 5' TCAACCAGCAATTCCAATACC & $59^{\circ} \mathrm{C}$ & 135 \\
\hline & 3' ATTCCTACCACCAGCAACC & & \\
\hline \multirow[t]{2}{*}{$18 s$} & 5' GCAAATTACCCACTCCCGAC & $55^{\circ} \mathrm{C}$ & 32,135 \\
\hline & 3' CGCTCCCAAGATCCAACTAC & & \\
\hline \multirow[t]{2}{*}{ Aggrecan } & 5' ACAGGTGAAGACTTTGTGGAC & $61^{\circ} \mathrm{C}$ & 135 \\
\hline & 3' AGTCAGTGAGTAGCGGGAGG & & \\
\hline \multirow[t]{2}{*}{ ADAMTS4 } & 5' AGGAGGAGATCGTGTTTCCAGAGA & $55^{\circ} \mathrm{C}$ & 135 \\
\hline & 3' AAAGGCTGGCAAGCGGTACAACAA & & \\
\hline \multirow[t]{2}{*}{ ADAMTS5 } & 5' TTCGACATCAAGCCATGGCAACTG & $55^{\circ} \mathrm{C}$ & 135 \\
\hline & 3' AAGATTTACCATTAGCCGGGCAGG & & \\
\hline
\end{tabular}

\section{Results}

\section{Influence of IL-1RA treatment following dynamic compression of meniscal explants}

Gene expression with and without IL-1RA following dynamic compression

Although trends within genes were similar between superficial and deep explants, only superficial zones unveiled a significant response to IL-1RA treatment. A significant decrease was observed in expression of IL-1 $\alpha$, MMP-3, MMP-13, and ADAMTS-4 genes for superficial explants treated with IL-1RA and exposed to large dynamic strains compared to untreated explants (Figure 3-1). ADAMTS-4 was not only significantly decreased at large dynamic strains, but demonstrated a significant decrease in the 
superficial zone following all three levels of dynamic loading when treated with IL-1RA compared to untreated. Interestingly, COX-2 gene expression in the superficial zone was significantly upregulated following IL-1RA treatment with $0 \%$ dynamic loading compared to the untreated explants (Figure 3-2). Although iNOS showed a trend toward decreased expression with IL-1RA treatment, this change was not significant. There was no significant difference in Aggrecan gene expression between IL-1RA treated and untreated explants for any dynamic compression level (data not shown).

\section{Discussion}

The present study is the first of its kind to explore the endogenous role of the IL-1 pathway on knee meniscal biochemistry following dynamic loading. The effects of mechanical loading alone on meniscal explants have been previously investigated in several studies. Specifically, the role of mechanical loading on the inflammatory ${ }^{32}$ and catabolic ${ }^{174}$ responses of this soft tissue have been suggested to contribute to its degradation and inhibited healing response. Previously, we have shown that a strain magnitude dependency exists for the expression of IL-1 $\alpha$, as well as select MMPs and ADAMTS molecules, in meniscal explants following dynamic compression ${ }^{32}$. Additionally, previous research has established the influence of exogenous IL-1 on the breakdown of cartilage ${ }^{210-212}$ and meniscus ${ }^{139}$ in vitro. For example, exogenous IL-1 added to the media during incubation of explants has been shown to increase aggrecanolysis ${ }^{210}$ and inflammatory mediators ${ }^{213}$ in cartilage. With the present study, the relationship between endogenous IL-1 and dynamic compression in the potential degradation of the meniscus is suggested.

The present study investigated the role of IL-1RA on the transcriptional behavior of meniscal explants in vitro, particularly of the catabolic nature. It has been previously established that the activation of IL-1 leads to a cascading effect on the catabolic response of soft tissues ${ }^{130,214}$ (Figure 3-3). The role of IL-1RA in antagonizing the catabolic cascade of IL-1 is reinforced in this study. It is likely that individual cells express IL-1 $\alpha$ in a strain-dependent manner, yet the secretion of IL-1 $\alpha$ and its 
perpetuating influence on surrounding cells is blocked by IL-1RA (Figure 3-3). Therefore, although the endogenous expression of individual cells is likely not inhibited by IL-1RA, the cascading influence of IL-1 on the catabolic response of the meniscus was shown to be efficacious in this study.

Previous work by Gupta et al demonstrated that the endogenous response of meniscal explants involves the transcription of the $\alpha$-form of IL-1, yet the $\beta$-form may not be transcriptionally activated following dynamic compression of this particular tissue in vitro ${ }^{32}$. Based on such findings, the present study only measured the relative gene expression of IL-1 $\alpha$. Differences in the two isoforms lie in their maturation modes ${ }^{215}$ and affinity for IL-1 receptors ${ }^{216}$. The activation of these two cytokines is dependent on cell derivation ${ }^{217}$, and stark differences between the two isoforms in gene expression regulation, stability, and secretion make these molecules quite distinct from each other 218 . The nature of IL-1 $\beta$ and its expression following mechanical loading may have been elucidated in expression profiles of the culture media, as this molecule is in its active form extracellularly. However, in this and previous studies, RNA was not harvested from the media ${ }^{32,135}$. Future studies investigating gene expression of both tissue and media may identify the relationship of strain-dependent expression of both IL-1 $\alpha$ and IL-1 $1 \beta$.

It is interesting to note that in the current study, the superficial zone demonstrated a transcriptional response to IL-1RA treatment following large dynamic strains different than that of its deep counterpart. This could in part be due to differences in cell type populations between superficial and deep zones ${ }^{58}$. Previous works have investigated the differences in gene expression between deep and superficial locations in the meniscus $^{32,135}$. In a partial meniscectomy model, Kobayashi et al found a distinct influx of nitrotyrosine in the superficial zones compared to the same zone in sham operated rabbits $^{11}$. It is likely that the superficial and deep zones of the meniscus respond differently; however, the data presented here should be taken with caution, as the gene expression of MMP-1, -3, ADAMTS-4, and ADAMTS-5 from deep explants did appear to be influenced by IL-1RA treatment, although such changes were not statistically significant. Additionally, it is possible that the strain applied to the whole explant was not equivalent throughout the depth of the tissue. Gupta et al demonstrated using finite 
element modeling that the size, shape, and position of an individual cell in an explant dictates its localized mechanical environment ${ }^{118}$. It is possible that the superficial zone saw more strain than the deep zone. Also, the compliance of the tissue may have varied throughout the depth of the explant potentially due to differences in mechanical stiffness between zones. However, differences in micromechanical stiffness between different depths of the menisci are not known. Future studies should investigate how changing the boundary conditions of dynamic loading on meniscal tissue can influence the depthdependent biochemical and mechanical nature of this tissue.

The use of corticosteroids ${ }^{219}$, NO inhibitors ${ }^{111}$, and MMP inhibitors ${ }^{140}$ have been previously shown to reduce the catabolic effects of IL-1 in vitro. However, none of these treatments have been completely efficacious in blocking IL-1 activity and subsequent catabolic-induced damage completely, likely because they act downstream of initial IL-1 binding ${ }^{218}$. The results of the present study illustrate the strong influence of the preemptive blockade of IL-1 on MMP and aggrecanase expression, as well a trend to reduce GAG released to the media, following a single bout of large dynamic compression. Even still, the complete blockade of NO release was not illustrated in the present study following treatment with IL-1RA.

The release of $\mathrm{NO}^{135}$ and $\mathrm{iNOS} / \mathrm{COX}-2$ gene expression of meniscal explants, although not influenced by treatment with IL-1RA, may elucidate a different pathway involved with meniscal mechanotransduction and degradation. Other researchers have suggested the critical role of COX-2 in the pathogenesis of OA ${ }^{175}$. Although IL- $1 \beta$ is known to induce NO release ${ }^{220,221}$ and COX-2 production ${ }^{222}$ in fibroblast/chondrocyte cells, there are other established pathways that can be involved. For example, others have shown that the induction of COX-2 and iNOS production occurs following treatment of chondrocytes with advanced glycation end products (AGE), and NO release can be influenced through the extracellular signal regulated protein kinase (ERK) and c-jun Nterminal kinase (c-Jun) pathways ${ }^{223}$. Such findings may suggest a role of alternate modalities in fibrochondrocytes in the degradation of this soft tissue. It is also possible that the expression of iNOS and COX-2, as well as the release of NO, in the present study 
may have peaked prior to the $24 \mathrm{hr}$ post-incubation period and subsequently dropped below levels which may have indicated upregulation and increased production which were missed in this study.

Clinical treatments of OA with anakinra, a recombinant form of IL-1RA, have not demonstrated severe adverse effects to patients ${ }^{150}$, suggesting its safety and tolerance as a form of intra-articular treatment. Unfortunately, clinical investigations using anakinra in patients with OA have not proved to be beneficial in sidelining the progression of degeneration and pain associated with the disease ${ }^{150}$. It is possible that the delayed intervention with IL-1RA or other anti-inflammatory therapies until after OA is diagnosable may be a futile attempt. Interestingly, genetic screening for IL-1RA haplotypes may provide information to clinicians about high- and low-risk patients and may also suggest IL-1 activity as a determinant for OA severity in the knee ${ }^{224}$. Both the initial pro-inflammatory response following trauma and the risk factors associated with OA development must therefore be further investigated to elucidate the nature of soft tissue degeneration. Nonetheless, these investigations, along with the present study, may suggest an alternative pathway of tissue degradation in addition to the IL-1 pathway.

\section{Conclusions}

This is the first study of its kind to explore the role of endogenous IL-1 on meniscal explants following various levels of dynamic compression. A significant down-regulation of several catabolic molecules following IL-1RA treatment was uncovered, underlining the endogenous response of the meniscus itself to the IL-1 pathway. However, it was interesting that previous work did not find a significant influence of IL-1RA on the release of NO from meniscal explants into the media ${ }^{135}$, which was reflected in the present study using indirect methods (iNOS gene expression). Pathways other than IL-1 are likely involved in the catabolic cascade that leads to tissue degradation, and various cell types of tissues in the knee may behave differently as well. Therefore, future investigations using an in vivo model are needed. 


\section{Recommendations}

Long-term mechanotransductive response of isolated meniscal tissue should be investigated in order to better characterize this tissue's role in inflammation and development of OA. Often, this tissue is overlooked as a contributor to the development and progression of $\mathrm{OA}$, although it is well known that preservation of meniscal integrity is beneficial in delaying knee joint degeneration. Additionally, it is known that the meniscus is an inhomogeneous and anisotropic material, yet few have investigated regional differences in its mechanical properties and biochemistry. Therefore, region- and strain-dependent characterization of the meniscal biochemistry with and without IL-1RA should be performed.

(next page) Figure 3-1. Relative expression of catabolic genes for both superficial and deep explants with and without IL-1RA treatment. * = significant difference in expression at $0 \%$ strain for same treatment; $\wedge=$ significant difference in expression at $10 \%$ strain for same treatment; \# = significant difference in expression at $20 \%$ strain for same treatment; arrow = significant difference in expression between treatment groups; n.d. $=$ no detectable gene expression . 

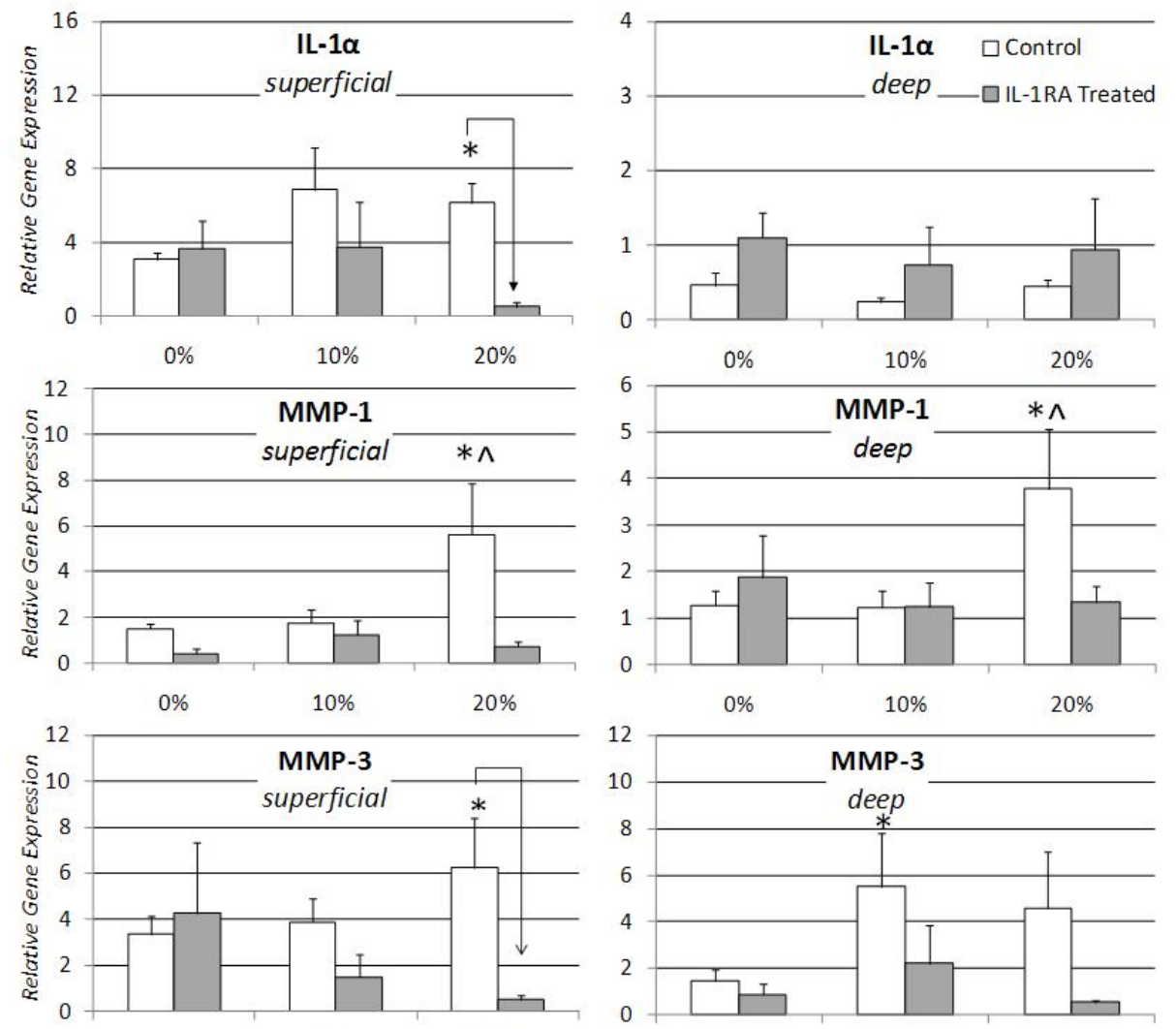

$0 \%$

$10 \% \quad 20 \%$

$0 \%$

$10 \%$

$20 \%$
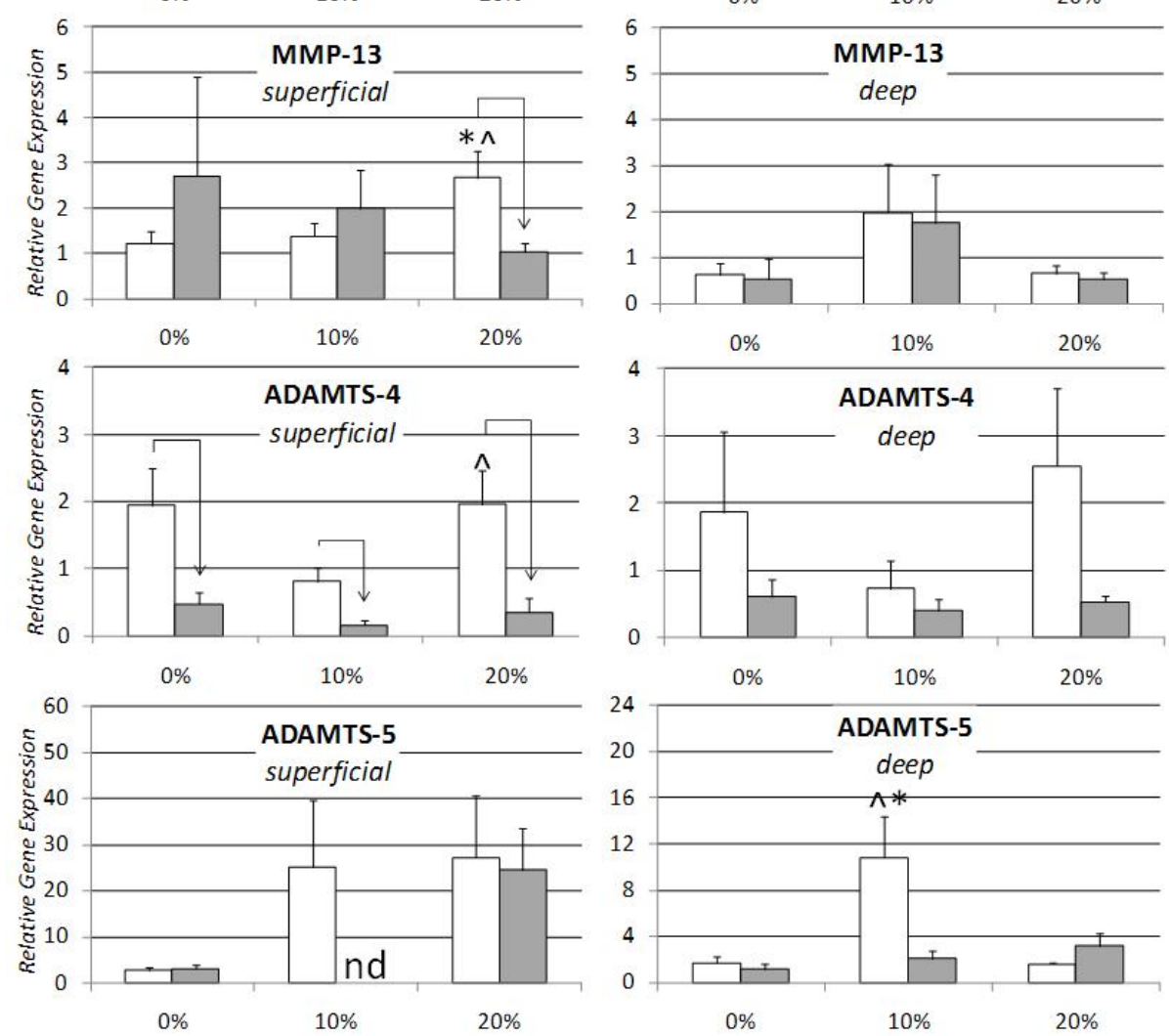

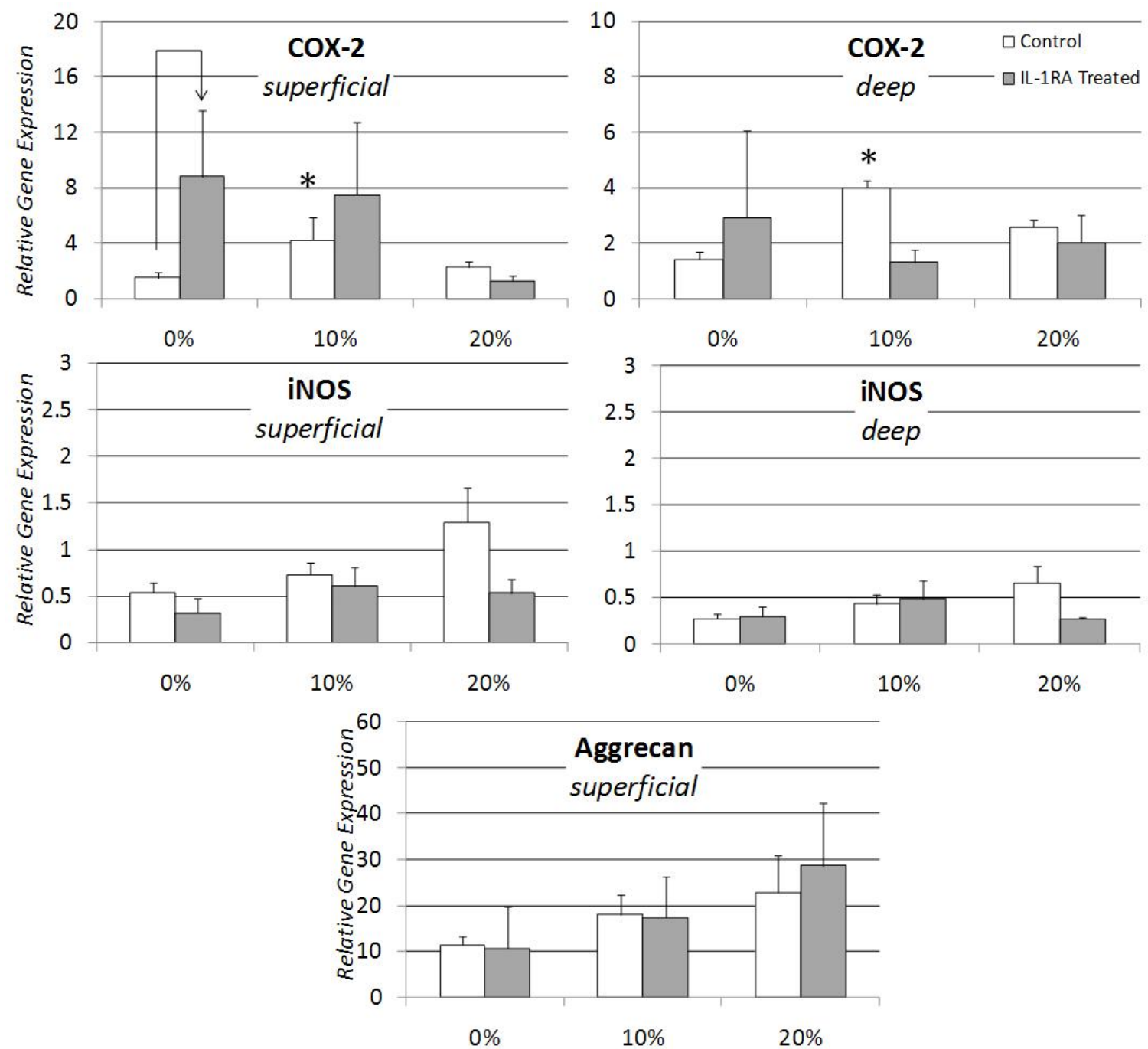

Figure 3-2. Relative expression of COX-2and iNOS, genes for both superficial and deep explants, as well as aggrecan gene for superficial explants only, with and without IL-1RA treatment. $*$ = significant difference in expression at $0 \%$ strain for same treatment; arrow $=$ significant difference in expression between treatment groups. 


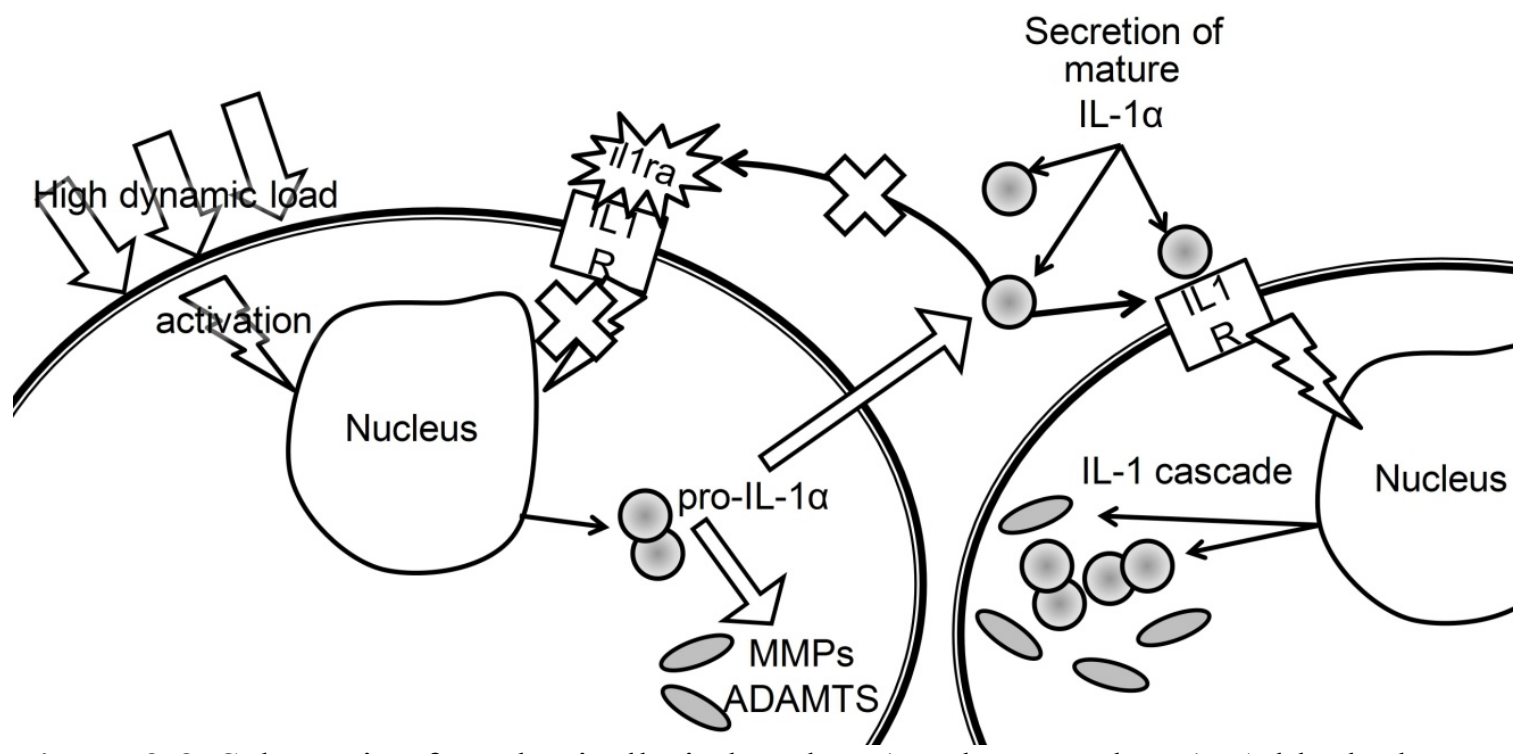

Figure 3-3. Schematic of mechanically-induced IL-1 pathway and IL-1RA blockade. 


\title{
Chapter 4 - Spatial Distribution of Sulfated GAG in Healthy Lapine Menisci ${ }^{3}$
}

\begin{abstract}
Introduction: The menisci have crucial weight-bearing roles in the knee. Regional variations in structure and cellularity of the meniscus have only been minimally investigated. Therefore, the goal of this study was to illustrate the regional cell density, tissue area, and structure of healthy lapine menisci.

Methods: Skeletally mature Flemish Giant rabbits were used for this study. Upon sacrifice, menisci were removed, fixed in formalin, and cryosectioned. Histological analysis was performed for the detection of sulfated glycosaminoglycans (GAG), collagen types I and II, cellular density, and tissue area. ANOVA and paired t-tests were used for testing of statistical significance.

Results: Glycosaminoglycan coverage of the medial meniscus significantly varied between regions, with the anterior region demonstrating significantly more GAG coverage than the posterior region. Inter- and intrameniscal comparisons revealed variations between zones, with trends that outer zones of the medial menisci had less GAG coverage. Collagen types I and II had marked characteristics and varying degrees of coverage across regions. Tissue area varied between regions for both medial and lateral menisci. Cellular density was dependent on region in the lateral meniscus.

Conclusions: This is the first study to illustrate regional and zonal variation in glycosaminoglycan coverage, size, and cellular density for healthy lapine meniscal tissue. This data provides baseline information for future investigations in meniscal injury models in rabbits.
\end{abstract}

\footnotetext{
${ }^{3}$ The material contained in this chapter has been published in the journal The Anatomical Record. Reprinted with permission.
} 


\section{Introduction}

Menisci are C-shaped fibrocartilagenous structures that have crucial weight-bearing roles in the knee, including shock absorption, joint lubrication, and proprioception 55, 100. Menisci distribute load between the femur and tibia, as the triangular cross-section of this tissue aids in joint alignment and stabilization ${ }^{86,225,226}$. During compression, the extracellular matrix of the meniscus has biphasic properties to help in cushioning and lubrication of the joint. This function is attributed mainly to the network of collagen fibrils and hydrophilic nature of glycosaminoglycans (GAG) within the tissue ${ }^{227-230}$. Damage or removal of the meniscus has been shown to lead to osteoarthritis (OA) ${ }^{231-233}$.

For over 30 years, animal models have been used to study the etiology of diseases as well as potential repair and/or replacement strategies ${ }^{234}$. The rabbit has frequently been used as a model for $\mathrm{OA}^{235}$. Recently, a lapine model has been developed to investigate the influence of traumatic impaction and ACL rupture on the remaining soft tissue structures of the kne $\mathrm{e}^{235}$. This study showed macroscopic damage to the menisci following traumatic knee injury, with the extent and location of meniscal damage differing compared to the surgically transected ACL model. However, prior to assessing further changes in meniscal tissue structure using this in vivo animal model, it is important to first characterize the native tissue structure.

The meniscus is an inhomogeneous material made several different matrix molecules including glycosaminoglycans (GAG), collagens, and proteoglycans. Although differences in regional material properties have been investigated in the meniscus ${ }^{236}$, regional variations in structure and cellularity have only been minimally investigated in the meniscus $80,83,236,237$. Additionally, most researchers focus mainly on medial menisci $79,159,238-242$, even though acute injuries can occur in both medial and lateral menisci ${ }^{243-}$ 248. Similarly, although the meniscus is inhomogeneous, region-dependent tissue variations in matrix molecules have only been briefly discussed ${ }^{83,249,250}$. It is well known that the menisci contain mostly type I collagen and that type II collagen is also present in the inner, hyaline-like zone ${ }^{251-254}$. However, the distribution of these two important 
macromolecules is not well understood across regions and zones of both medial and lateral menisci. Meniscal cell type has been documented to be zone-specific for human

and rabbit ${ }^{57,58}$, yet the density and distribution of cells in the meniscal body has not yet been investigated for any species. Unfortunately, the relationship between meniscal cellular density and tissue structure is not well understood ${ }^{60,117,236,237,255,256}$. This is likely due to the various types of animal models used in meniscal research ${ }^{236,253}$. Knowing the architecture and cell density is vital in understanding potential injury response and repair following meniscal damage. Therefore, the goal of this study was to illustrate the regional cell density, tissue area, and collagen/GAG structure of healthy lapine menisci. Understanding the distribution of cell density and glycosaminoglycans in the normal lapine meniscal tissue will help elucidate findings following various degrees of degeneration and degradation in rabbit OA models.

\section{Materials and Methods}

Five skeletally mature Flemish Giant rabbits were used for this study. The experiment was approved by Michigan State University's Institutional Animal Care and Use Committee. Animals were sacrificed at 6-12months of age using $85.9 \mathrm{mg} / \mathrm{kg}$ BW Pentobarbital I.V. Left and right menisci of both limbs were freed of ligamentous attachments and synovium. Immediately after removal, menisci were rinsed in PBS and then fixed in $10 \%$ formalin. After fixation, whole menisci bodies were then fixed in OCT-30\% sucrose blend (Pelco Int., Redding, CA, USA) for 48- 72hours. After OCT fixation, the menisci bodies were sectioned into anterior $(\mathrm{A})$, central(C), and posterior $(\mathrm{P})$ regions using a custom cutting tool designed with three blades permanently fixed at $45^{\circ}$ angles with respect to each other (Figure 4-1). Sections were covered with OCT embedding matrix, and then flash frozen with liquid nitrogen. Six (6) $\mu \mathrm{m}$ cryosections were obtained parallel to sectioning surface and subsequently stained for sulfated glycosaminoglycans (GAG), collagen I, collagen II, or cell nuclei. 


\section{Detection of Sulfated GAG}

Slides were stained using Fast Green-Safranin O (SafO) solution for detection of GAG coverage. With FG-Saf O staining, mucins and cytoplasms stain blue-green, nuclei stain black, and sulfated GAGs stain red. Sections were then imaged using an Olympus AX70 Microscope and DP70 camera. Red coverage of SafO staining was semi-quantitatively analyzed using Image J software (NIH, Bethesda, MD). Briefly, color images were converted to R-G-B stack and viewed as a grayscale image under green stack. With green stack, tissue appeared light and SafO positive stained regions appeared dark. Images were analyzed using the threshold function with a black to red ratio of at least 1:3. The aggregate percentage of area (SafO-positive) was then measured for each image in the following zones: inner $1 / 3$, middle $1 / 3$, outer $1 / 3$, as well as the entire section, for slides obtained from the anterior, central and posterior regions. Briefly, the total length of the meniscal section was measured using a line in ImageJ from the innermost point of the section and extended to the outer edge, halfway between the femoral and tibial surfaces of the meniscus. Then, the line was shortened to one-third the original length. Original images were used to verify correct threshold of red pixels in the R-G-B stacked images.

\section{Collagen Type I and II Distribution}

Immunohistochemistry was performed with antibodies raised against collagen types I and II (Medicorp, Montreal, Quebec). The immunohistochemical localization techniques for

collagen types I and II have been described previously ${ }^{257}$. Briefly, sections were sequentially digested with pronase $(1 \mathrm{mg} / \mathrm{ml})$ and hyaluronidase $(10 \mathrm{mg} / 1)$ for 30 minutes each at $37^{\circ} \mathrm{C}$. Sections were then blocked with pre-incubation buffer $(10 \%$ goat serum, $1 \%$ bovine serum albumin in PBS) at room temperature for 2 hours. Sections were incubated overnight with monoclonal mouse anti-human antibodies against collagen types I (1:400) and II (1:5000) (Medicorp, Montreal, Quebec). Biotinylated goat antimouse secondary antibodies were detected with Streptavidin-labeled AlexaFlour 488 (Invitrogen, Carlsbad, CA) and were additionally stained using propidium iodide (1:500 in PBS) for cellularity. Negative controls were determined using the above methods without incubation with the primary antibodies. A positive control was used for both 
antibodies on serial sections of human ligamentous tissue and articular cartilage, which are mostly collagen type I and collagen type II, respectively. Each section was then imaged using fluorescence microscopy and analyzed under blind conditions.

\section{Cell Density and Tissue Area Measurements}

Cells were detected using a propidium iodide staining technique and photographed using an Olympus AX70 microscope and DP70 camera. In brief, slides were placed in Coplin jars and fixed using $4 \%$ paraformaldehyde (PFA). Next, slides were washed using PBS (pH 7.4), removed from Coplin jars, and dried. Tissues were covered by a proteinase $\mathrm{K}$ dilution $(10 \mathrm{mg} / \mathrm{ml})$ and fixed again using $4 \%$ PFA. Slides were then immersed in a propidium iodide solution ( $1 \mu \mathrm{g} / \mathrm{ml}$ in PBS) to stain for cell nuclei. Each section was then imaged using fluorescence microscopy. Image analysis using CellC (Tampere University of Technology, Tampere, Finland) was performed to determine total cell count using an empirically determined cluster size. MetaMorph imaging software (Molecular Imaging, Downington, PA, USA) was used to determine the tissue area of each region. Cell density was determined using the total cell count (cells) per tissue area $\left(\mathrm{mm}^{2}\right)$.

\section{Statistical Analysis}

Analysis of variance (ANOVA) was performed using Minitab 15 statistical software (Minitab Inc., State College, PA). Descriptive statistics were calculated for all measures. Paired t-tests were performed to determine differences between left and right limbs. Oneway ANOVA was performed for regional comparisons between medial and lateral menisci. One-way ANOVA was also performed within regions for zonal comparisons. Post-hoc t-tests with pair-wise comparisons within each animal of menisci (medial vs lateral) and regions (A vs C, A vs $\mathrm{P}$, and C vs $\mathrm{P}$ ) were used to determine regional and zonal differences. Preliminary analyses demonstrated no statistically significant differences between left and right limbs. Therefore, left and right limb data were averaged for respective regional and zonal comparisons. Statistical power was established to be $\geq 0.80$ with the sample size used in this study. 


\section{Results}

Glycosaminoglycan coverage was significantly higher in the anterior region $(24.44 \pm$ $8.65 \%)$ of the medial menisci compared to the central $(5.41 \pm 1.70 \%)$ and posterior $(4.35$ $\pm 2.29 \%$ ) regions (Figure 4-2). Within the medial menisci, the anterior regions showed greater GAG coverage compared to the central and posterior regions. Differences between zones in $\mathrm{A}$ and $\mathrm{C}$ regions of medial menisci were only significant for the outer region (Figure 4-3). Within the central region of the lateral menisci, the inner zone (2.28 $\pm 1.06 \%$ ) showed significantly less GAG coverage than the outer zone $(19.62 \pm 6.90 \%)$, with this same trend occurring in the lateral posterior region (Figure 4-3). The amount of GAG coverage between the central region of the two menisci (medial and lateral) was significantly different for two of the three zones; outer and inner zones. To note, the medial meniscus central region increased in GAG coverage moving from the outer zone to the inner zone, the opposite trend was seen for the lateral meniscus (Figure 4-3).

Distribution of collagen type I (Figure 4-4) and type II (Figure 4-5) varied qualitatively in both medial and lateral menisci. In general, medial and lateral menisci stained heavily positive for collagen type I throughout all regions. However, the deep body of the menisci sometimes lacked collagen type I staining. Outer zone and peripheral localization of collagen type I was apparent in all regions of both menisci (Figure 4-4).Collagen type II appeared to be primarily localized at the peripheral surfaces of both medial and lateral menisci. In some regions, this localization represented a distinct continuous thickness, especially in the central and posterior regions (Figure 4-5). Again, the deep body of the menisci seemingly lacked collagen type II positive staining (Figure 4-5).

Differences were observed between menisci in tissue area, with the lateral meniscus demonstrating significantly greater tissue area than the medial meniscus in the central and posterior regions (Figure 4-6). For both the medial and lateral menisci, the anterior and posterior regions demonstrated a significantly greater area compared to the central region (Figure 4-6). Furthermore, the anterior region was also significantly larger than the 
posterior region in the medial meniscus (Figure 4-6). Further investigation of tissue area through the outer, middle and inner zones of the menisci confirm the triangular crosssection of the menisci with the outer zone being significantly larger in area, with decreasing tissue area in the inner zones (Figure 4-7). In 6 of the 9 zones studied, the lateral meniscus was significantly larger than the medial meniscus (Figure 4-7).

Cell density measurements also differed across regions and zones. The posterior region of the lateral meniscus was significantly more cellular than any other region in both medial and lateral menisci, and this was the only region where the cell density between medial and lateral menisci significantly differed (Figure 4-8). Within the medial meniscus, the inner zone of the anterior region was more densely populated with cells than the same zone of the posterior region (Figure 4-9). The outer zones of the central and posterior region, as well as the inner zone of the posterior region, were significantly more cellular in the lateral menisci than the respective zones of the medial menisci (Figure 4-9). Cellular density between zones, within a given region, also varied (Figure 4-9). The outer and middle zones of the posterior region were more densely populated with cells compared to its respective inner zones (Figure 4-9).

\section{Discussion}

This is the first study to illustrate the complete regional and zonal variation in glycosaminoglycan coverage, size, and cellular density of meniscal tissue in any species. This study emphasizes the importance of location-dependent histological properties of the lapine menisci. Future studies that investigate menisci of rabbits as well as other species should be mindful of the region and zonal differences between medial and lateral locations of this inhomogeneous tissue. In order to better understand currently implemented animal models used to investigate joint disorders such as degenerative joint disease, we must first understand the normal physiology and biology of tissues such as the meniscus. 
Sulfated glycosaminoglycans are charged sugar chains that adhere to proteins and attract water molecules. This provides the tissue with biphasic properties, as the water can exude from the tissue with rate-dependent characteristics. Therefore, understanding the distribution of sulfated GAGs is important in the characterization of the meniscus. In this study, we found that the anterior region of the medial meniscus demonstrated significantly higher coverage of sulfated-GAG positive staining, which likely influences the region-dependent mechanical properties of the menisci. Others have suggested regional variations in material properties across the medial meniscus, including greater stiffness in the anterior region of human and rabbit medial menisci ${ }^{236}$ and correlation between GAG content and compressive and storage moduli in human menisci ${ }^{80}$. Our results reflect similar distributions in GAG measures to Bursac et al's work and expand on their findings by including in-depth zonal comparisons of GAG coverage. The current study emphasizes the use of rabbits as an animal model for meniscal damage and joint malalignment is appropriate given the similarities between GAG coverage and mechanical properties between human and lapine menisci. Although the approach for determining GAG coverage from our implementation of histology is not a direct quantitative measure of GAG content, previous researchers have shown a strong correlation between our method and those using biochemical assays specific for sulfated $\mathrm{GAG}^{258}$.

The regional distribution of collagen type I and II has rarely been discussed in the literature ${ }^{252}$. For example, it is known that collagen fibrils at exterior surfaces of the menisci are circumferentially oriented, providing resistance to hoop stresses applied to the meniscus during loading ${ }^{259}$. The present study highlights the distribution of collagen types I and II through the lapine medial and lateral menisci. The heavy staining of collagen type I was to be expected, as collagen type I accounts for more than $90 \%$ of meniscal tissue collagen in other species $55,252,260$. However, the regional and zonal distribution of collagen type I in rabbits had not yet been explored through immunohistochemistry. It is also interesting to note that the distribution of collagen type II varies across regions and zones in the lapine menisci. In this study, we have shown 
qualitatively that collagen type II is distributed around the periphery of the tissue, suggesting its role as a cartilage-like boundary for the meniscal body. This study showed that the middle and inner zones of the medical meniscus contain more sulfated GAG, who negative charge attracts and holds water within the tissue. A reduced density of collagen Type I and II matrix, particularly in the middle region of the tissue, may provide the GAG with more space to create a meshwork for control of tissue permeability and hydration ${ }^{261}$. Although there may be a relationship between the collagen network and GAG coverage, we did not quantify collagen coverage or correlate the spatial distribution of collagen relative to GAG in the present study.

This study is the first to illustrate tissue area measurements of the lateral menisci for rabbit. Interestingly, this study observed that the central region areas for both the medial and lateral menisci were smaller than their anterior and posterior counterparts. This increased area in the anterior and posterior areas of the menisci is likely related to the fact that at the anterior and posterior horns the menisci attach to the underlying subchondral bone so that the circumferential hoop stress developed within the main body of the soft tissue meniscus can be easily transferred to the more rigid subchondral bone without rupture ${ }^{231,259}$. Additionally, as loading increases, as seen with developmental gait ${ }^{54}$, there is a need for an increase in the attenuation of load transmitted through the menisci to the underlying articular cartilage. Thus, it is possible that the meniscal tissue area may adapt and become thicker in certain regions to compensate for these increased loads. Further developmental studies are needed to explore these ideas. Others have reported the tensile strength in the anterior region of the medial menisci to be greater than the posterior $^{259}$. The lateral posterior attachment is unique in the rabbit as it attaches only to the medial femoral condyle ${ }^{259}$. Therefore, geometrical differences may play a role in the loading characteristics of the menisci, and vice versa.

The meniscus is an inhomogeneous material made of varying matrix molecules including collagen I and II, and the orientation and composition of these matrix molecules allows the material to behave differently depending on directional loading ${ }^{238,262}$. We chose to 
investigate the distribution of these two molecules because of the different roles these molecules play in biological tissues. For example, collagen type I resists tensile stresses, and supplies the meniscus with the ability to withstand hoop (or circumferential) stresses during loading. Collagen type II, which is the predominant collagen in articular cartilage, plays an important role in the compressive properties of tissues. The two different collagen types provide the menisci with the ability to perform a variety of mechanical functions. The distribution of these two important macromolecules likely plays a role in the tissue's mechanical properties, which may influence other aspects of the tissue investigated in this study. Previous research using rabbit models have shown that the lateral anterior region may be subjected to higher loads than the medial anterior region, causing an increase in contact pressure ${ }^{263}$. Lower observable cell density in the anterior region may be attributed to the increased stiffness of the tissue in the anterior region, as cell mobility and infiltration may be difficult through stiffer extracellular matrix ${ }^{236}$.

The importance of this study focuses on the future use of certain species in animal osteoarthritis models. Although OA is defined as the onset and progression of cartilage damage and degradation, the meniscus likely plays a crucial role in the disease's prevention and progression ${ }^{159,264-267}$. Altered loading, remodeling, and disuse can result in changes to the meniscus $38,112,113,268,269$. Commonly used as an OA animal model, the rabbit has demonstrated strong changes in meniscal morphology following anterior cruciate ligament transection (ACLT) ${ }^{10,159,161}$. By underlining the regional and zonal differences in cellularity, tissue area, and sulfated GAG coverage in the healthy rabbit, we will be able to explore how various OA models can influence such distributions. Previously, Sweigert et al demonstrated that porcine has a higher aggregate modulus in the anterior region of the medial meniscus compared to central and posterior regions ${ }^{236}$. Prior to this, Nakano et al showed that the anterior region of the porcine menisci demonstrates significantly higher GAG staining in this region ${ }^{237}$. Interestingly, some researchers have reported similar trends between rabbit and human compressive moduli for meniscal regions ${ }^{236}$. Such comparisons follow similar trends as the GAG coverage measured for rabbits in this study. However, recent work by Chevrier et al has 
comparatively assessed both rabbit and sheep as potential animals for use as repair models ${ }^{253}$. Their findings suggest the preferential use of sheep over rabbit for in vivo assessment of meniscal repair based on cross-sectional area, vascular penetration, and lamellar layer. However, rabbits remain a convenient and accessible animal for laboratory investigations in OA. Structurally, the rabbit model mimics meniscus characteristics similar to that of human, making it a valid model for future use in animal studies.

In summary, the use of animal models is important for laboratory-based investigations into trauma-induced injuries to the soft tissues of the knee. Our study outlines the distribution of sulfated GAGs, cellular density, and size of healthy rabbit menisci to be used for comparative purposes in future OA investigations. This is the first study to investigate the coverage of sulfated GAG and the density of cells in the various regions and zones of both medial and lateral menisci. The findings presented in this study will aid in future investigations of damage to the menisci and knee, and provides new metrics of which can be used to study longitudinal progression of meniscal characteristics associated with degenerative joint disease in a rabbit model. 


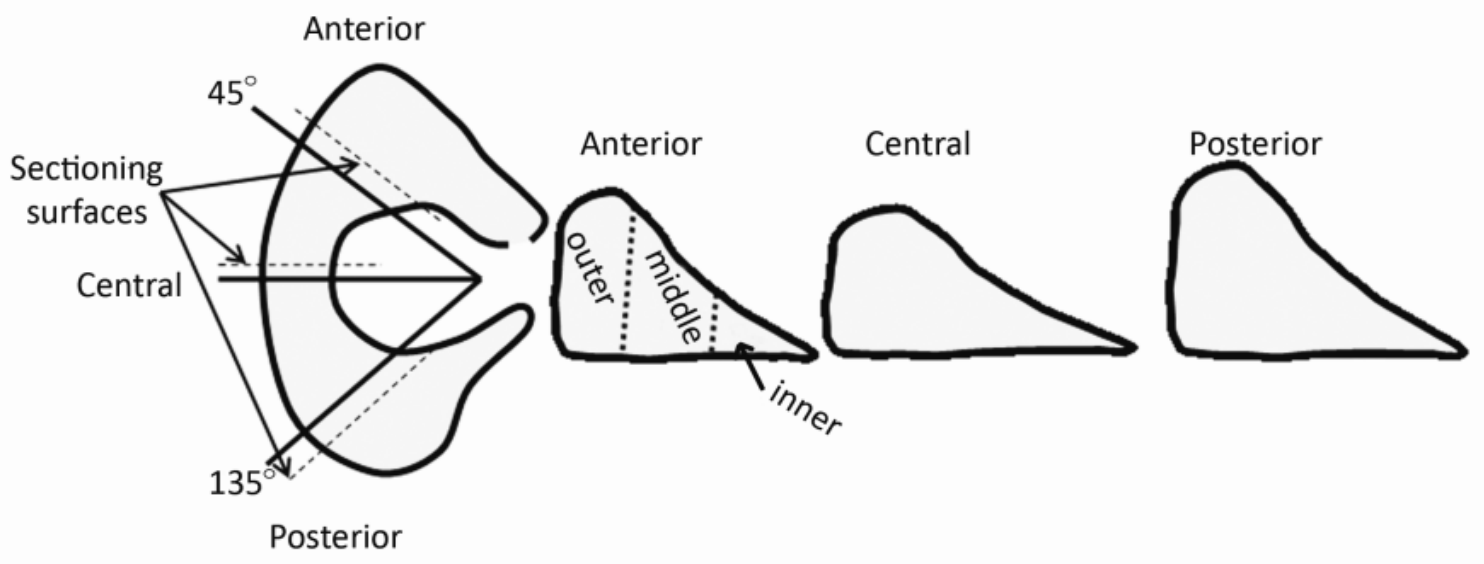

Figure 4-1. Sectioning of meniscal regions using a custom cutting tool assembled with blades at $45^{\circ}$ separation from each other. Zonal regions highlighted in anterior crosssection. 


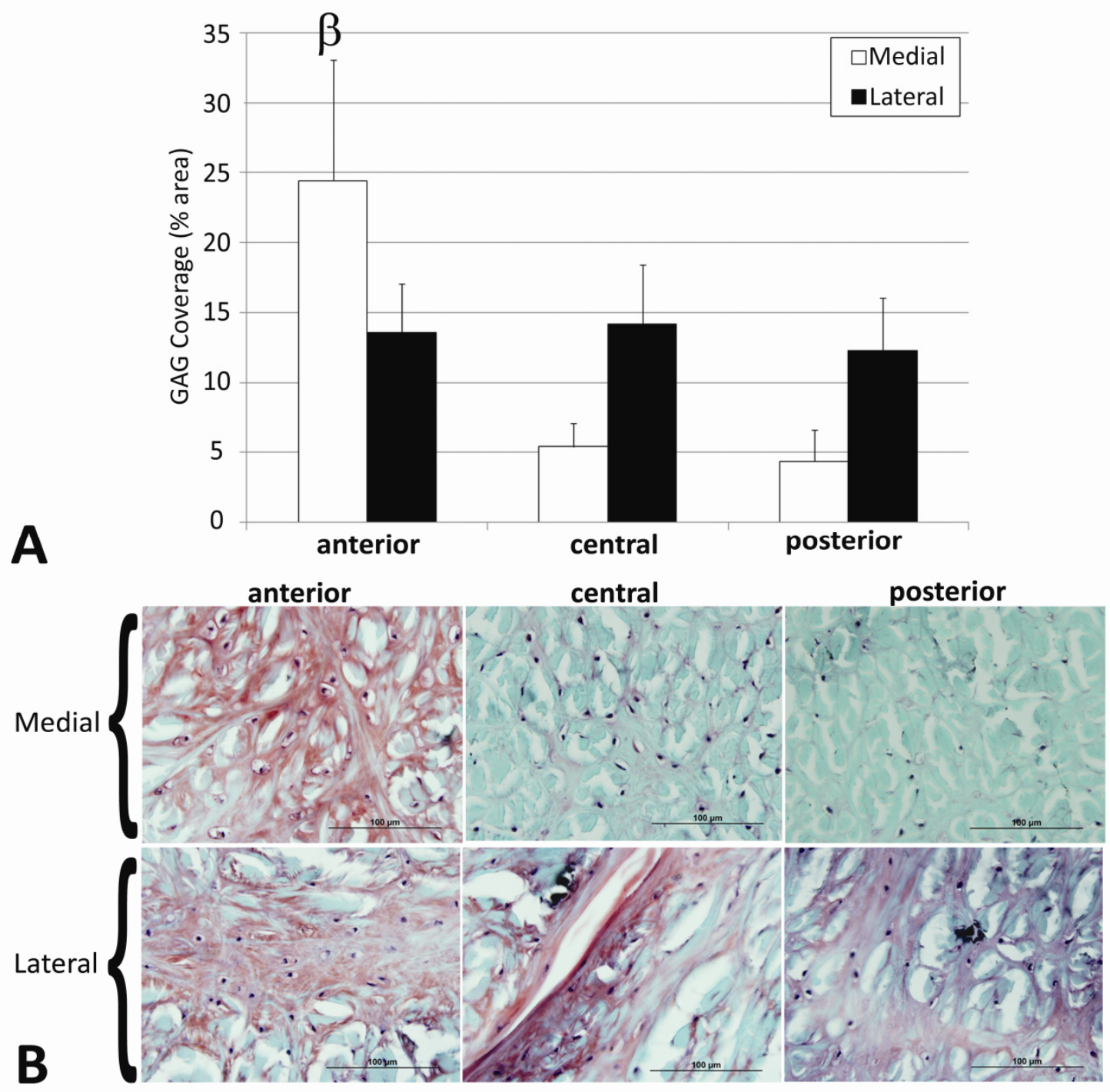

Figure 4-2. (A) Regional comparisons in percentage of GAG coverage between medial and lateral menisci and (B) representative SafO histological images.

$\beta=$ significantly different than other regions within same menisci. Scale bar is $100 \mu \mathrm{m}$. 

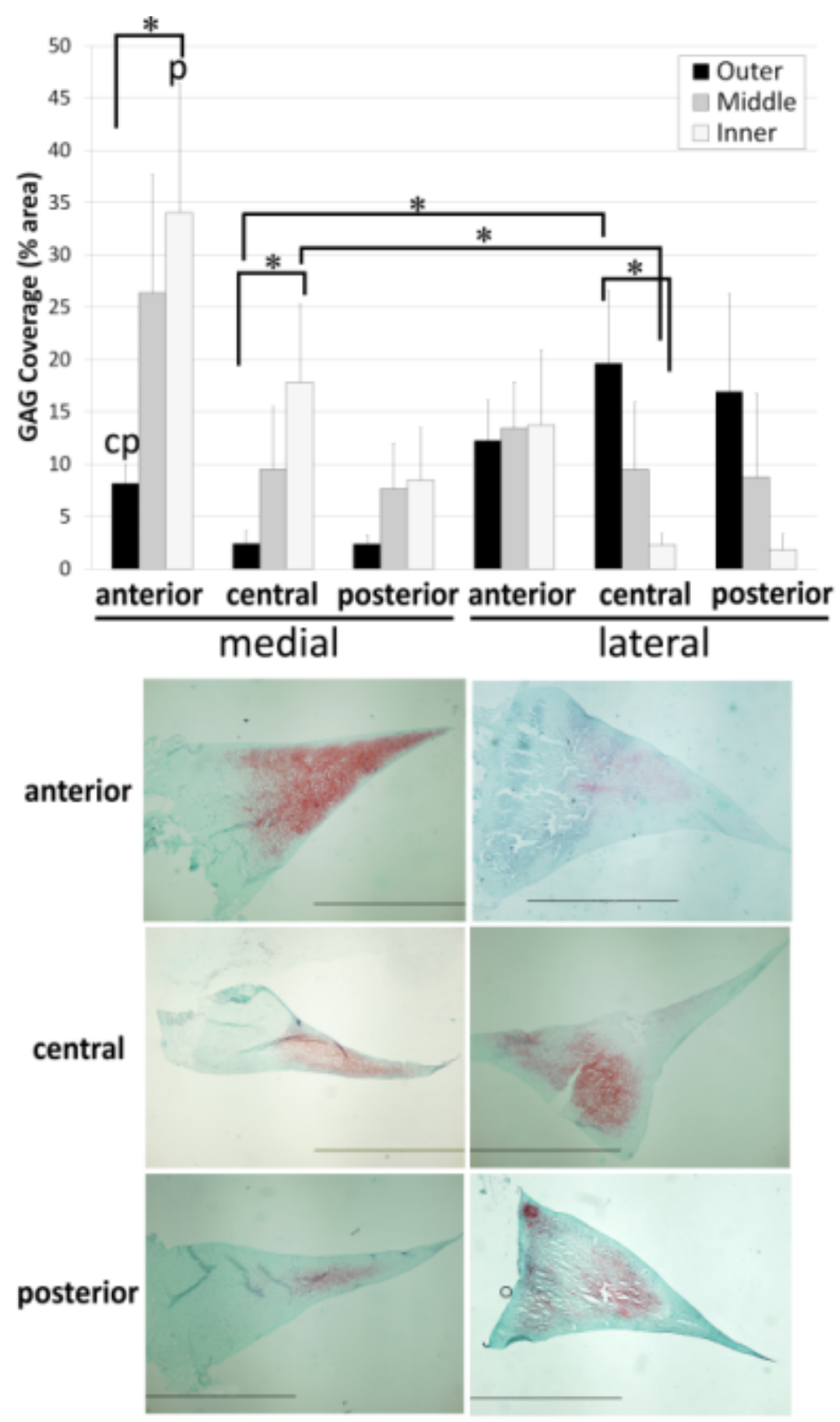

Figure 4-3. (A) Zonal comparisons of percentage of GAG coverage in medial and lateral menisci and (B) representative SafO histological images. $\mathrm{c}=$ significantly different than same zone of central region within the same menisci, $\mathrm{p}=$ significantly different than same zone in posterior region within the same menisci; $*=$ significantly different between zones. Scale bar is $2 \mathrm{~mm}$. 

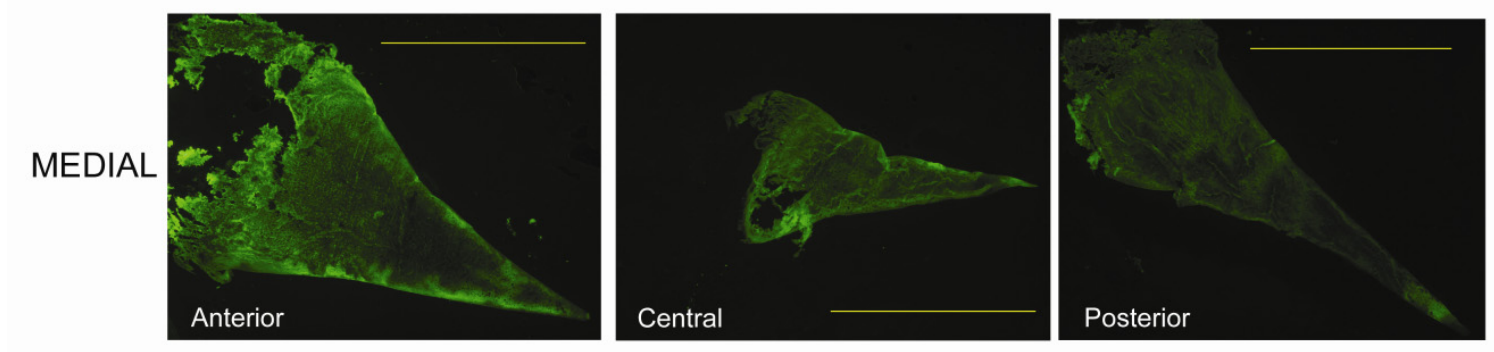

LATERAL
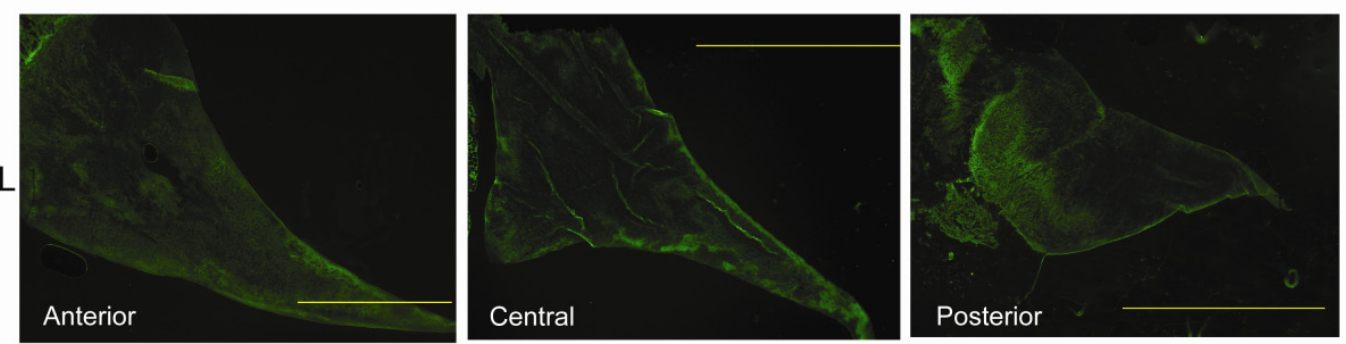

Figure 4-4. Collagen type I immuno-fluorescence staining of medial and lateral meniscal sections for anterior, central, and posterior regions. Scale bar $=2 \mathrm{~mm}$.

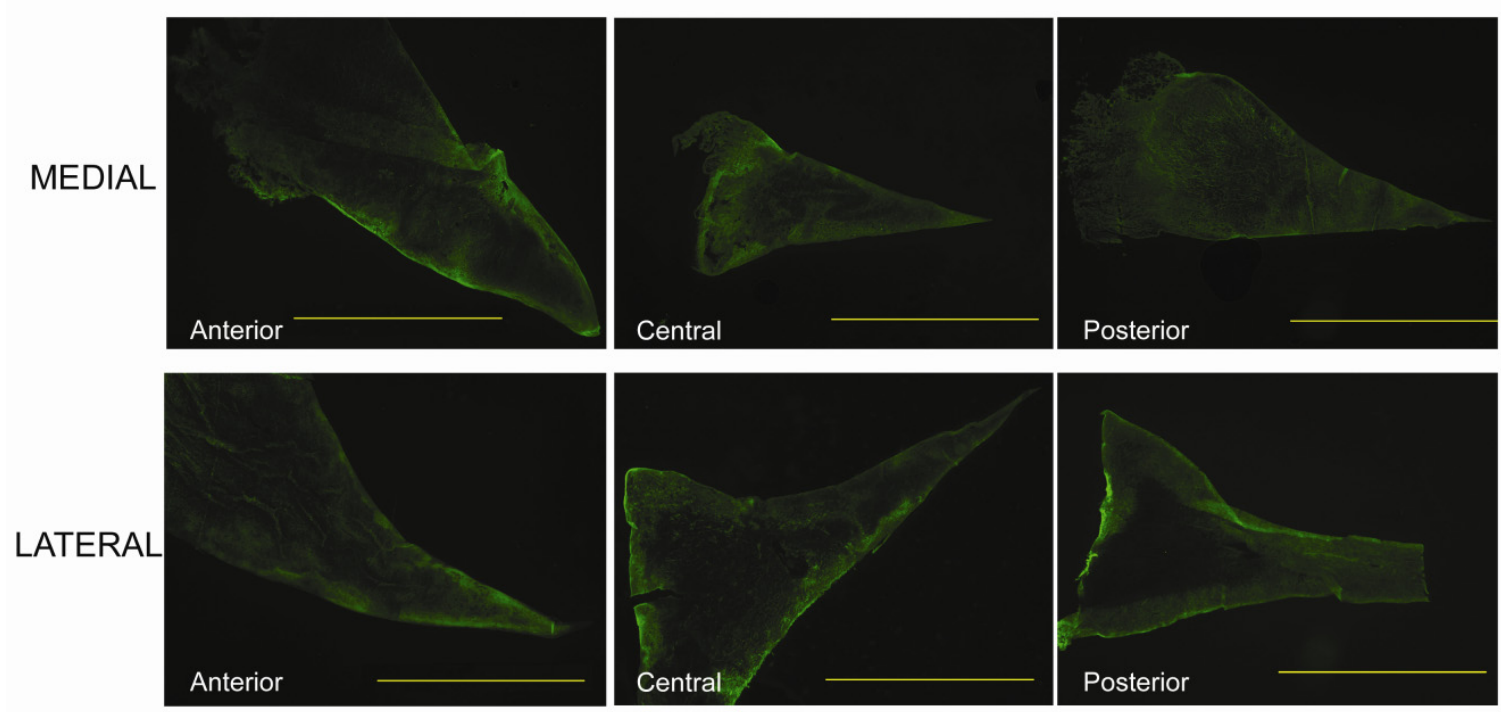

Figure 4-5. Collagen type II immuno-fluorescence staining of medial and lateral meniscal sections for anterior, central, and posterior regions. Scale bar $=2 \mathrm{~mm}$. 


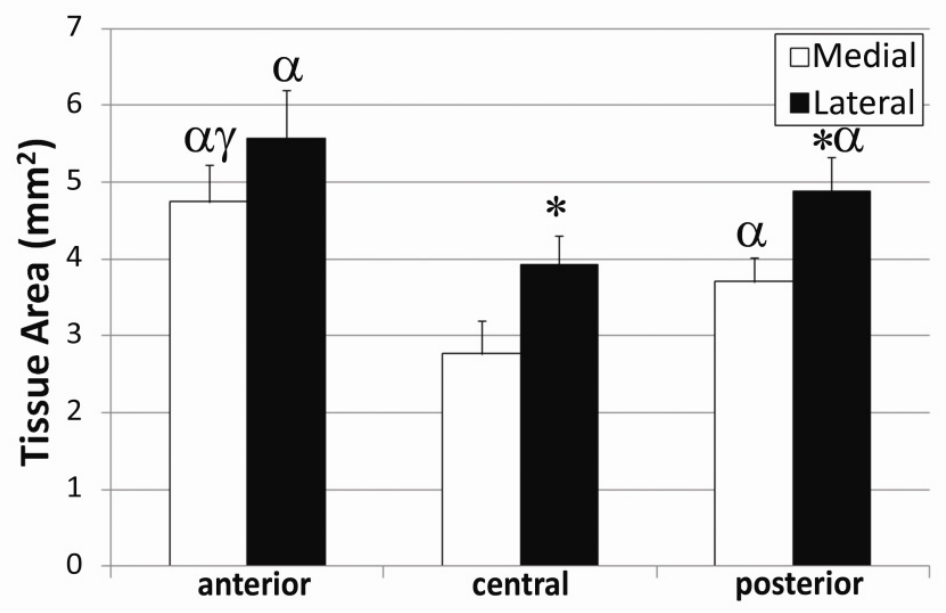

Figure 4-6. Regional comparisons in tissue area $\left(\mathrm{mm}^{2}\right)$ between medial and lateral menisci. * = significantly different than same region of medial menisci; $\alpha=$ significantly different than central region within same menisci; $\gamma=$ significantly different than posterior region within same menisci.

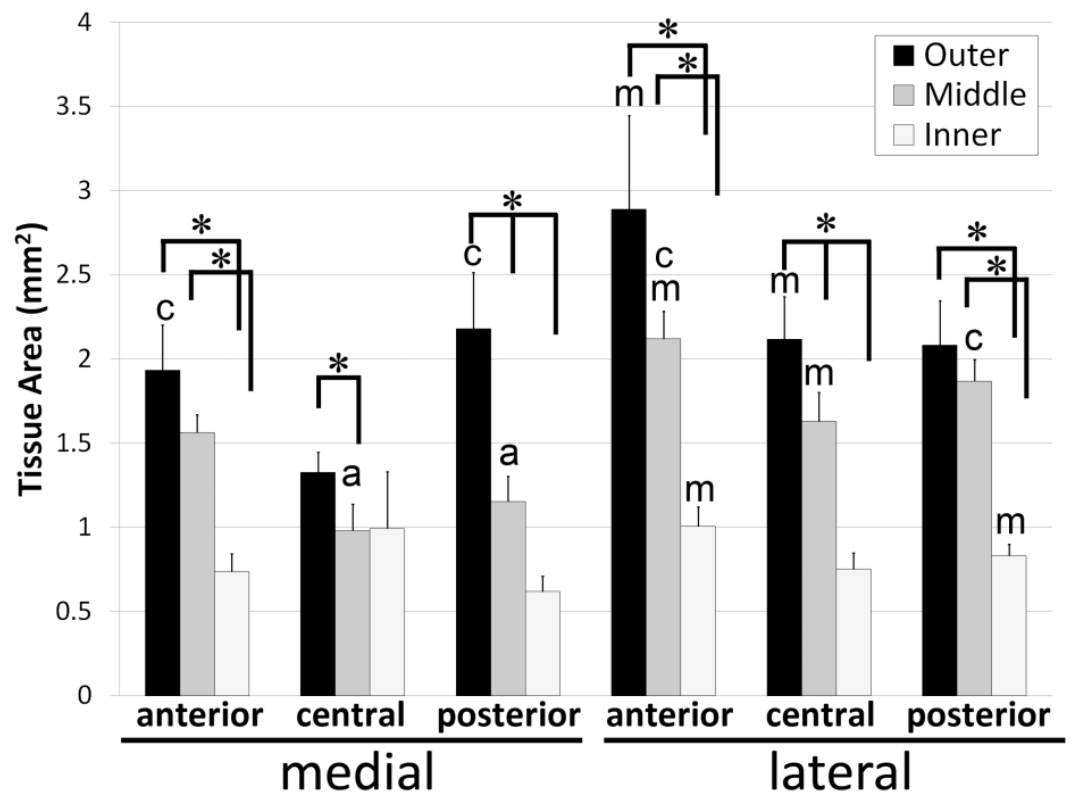

Figure 4-7. Zonal comparisons in tissue area $\left(\mathrm{mm}^{2}\right)$ between regions of the medial and lateral menisci. $\mathrm{m}=$ significantly different than same zone of medial menisci; $\mathrm{a}=$ significantly different than the same zone in the anterior region; $\mathrm{c}=$ significantly different than same zone of central region within the same menisci; * = significantly different between zones. 


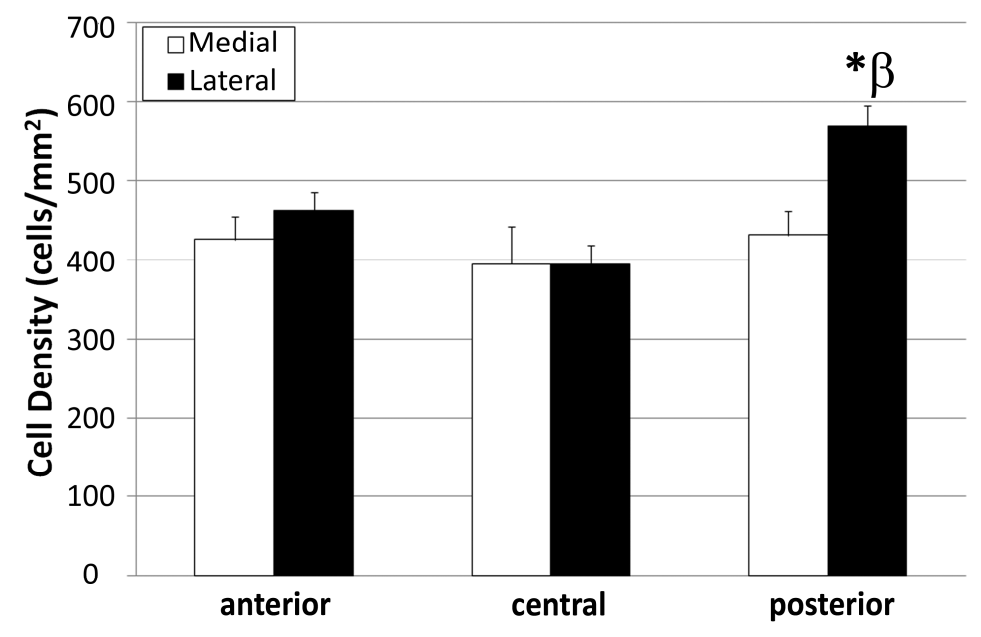

Figure 4-8. Regional comparisons in cell density $\left(\right.$ cells $\left./ \mathrm{mm}^{2}\right)$ between medial and lateral menisci. $\beta=$ significantly different than other regions of same menisci; $*=$ significantly different than same region of medial menisci

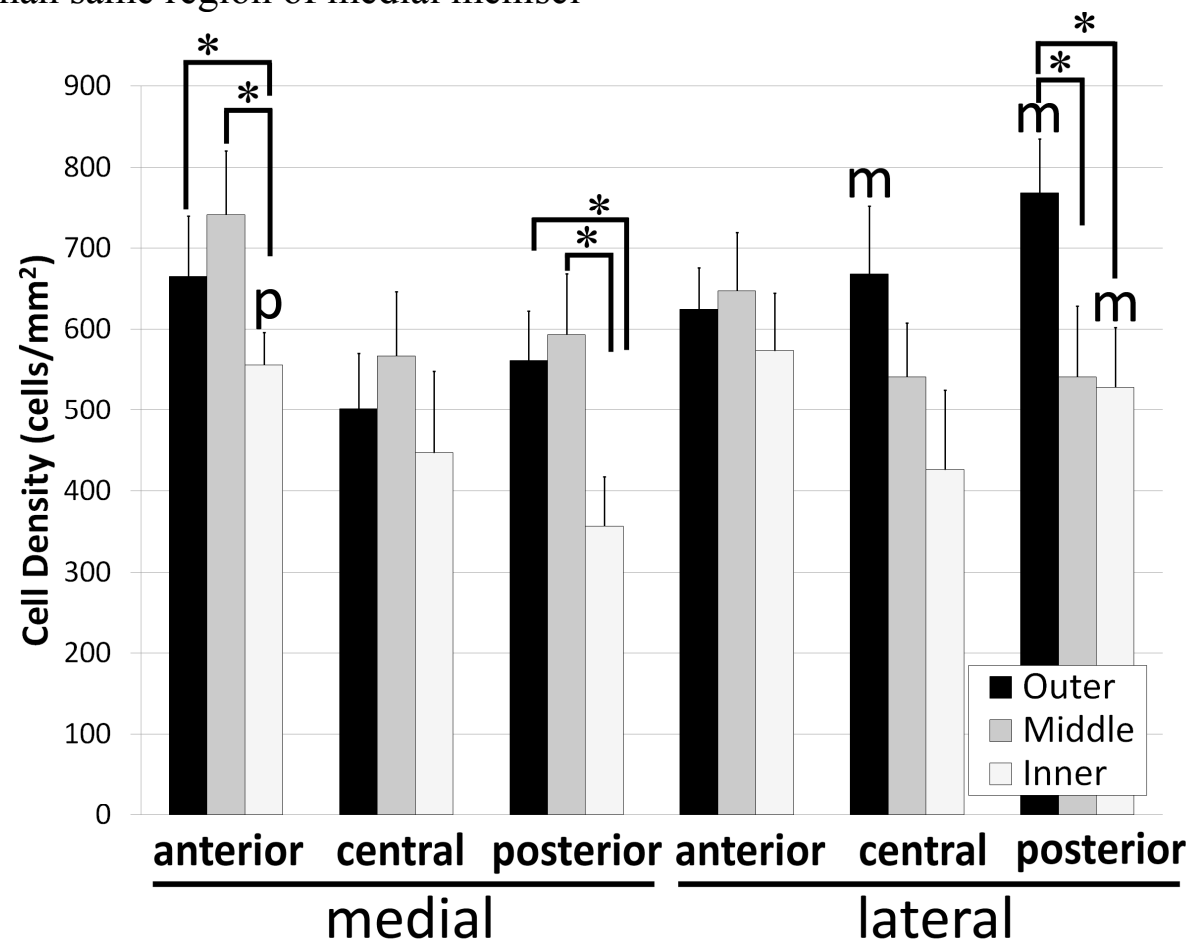

Figure 4-9. Zonal comparisons in cell density $\left(\right.$ cells $/ \mathrm{mm}^{2}$ ) between regions of the medial and lateral menisci. $p=$ significantly different than the same zone in the posterior region; $\mathrm{m}=$ significantly different than same zone of medial menisci; * = significantly different between zones. 


\section{Chapter 5 - Traumatic Anterior Cruciate Ligament Tear and its Implications on Meniscal Degradation ${ }^{4}$}

\section{Abstract}

Introduction: Injury patterns of the meniscus following impact trauma resulting in ACL rupture are not well understood. This study explored the spatial and temporal distribution of meniscal tears in a novel in vivo lapine model.

Methods: Rabbits were subjected to either tibiofemoral impaction resulting in ACL rupture or surgical ACL transection. Meniscal damage was assessed acutely and after 12weeks for traumatically torn, and after 12-weeks in ACL transected animals. Morphological grading was assessed using previously established criteria, and descriptions of meniscal damage were diagnosed. Histological assessment was also made on 12-week traumatically torn and ACL transected animals using Fast-Green/Safranin-O staining.

Results: Traumatic ACL rupture resulted in acute tears predominately in the lateral menisci. Animals subjected to both surgical transection and traumatic ACL rupture experienced degradation of the lateral and medial menisci 12 weeks after injury. However, traumatic ACL rupture resulted in acute lateral damage and chronic degradation of the menisci, as well as more severe degradation of the menisci 12 weeks after injury.

Conclusions: Both acute and chronic changes to the meniscus following traumatic impaction were observed. This research has implications for the future use of lapine models for osteoarthritis, as it incorporates traumatic loading as a more realistic mode contributing to the progression of OA compared to surgically transected models.

\footnotetext{
${ }^{4}$ The material contained in this chapter was previously published in the journal Journal of Surgical Research. Reprinted with permission.
} 


\section{Introduction}

The menisci play a crucial role in the dynamics of the knee. Their shape, attachment, and material properties contribute substantially to joint alignment and load transmission by distributing both tensile and compressive forces. Damage to the menisci can influence proprioception, stability, and mobility of the knee $231,239,240,270,271$. Risk factors of meniscal tears include prolonged or repeated deep knee bending, obesity, and sports ${ }^{17}$. Acute injury, as seen in alpine sports, involves complex dynamics which can damage singular or multiple tissue structures of the $\mathrm{knee}^{17,272}$. Meniscal tears are typically thought to be initiated by coupled compression and twisting movements ${ }^{273,274}$, which can accompany high-energy maneuvers such as cutting, jumping, and landing during sporting events $^{275}$. It is not uncommon for meniscal injuries to occur in conjunction with ACL lesions, and the loading imbalance that results in ACL lesions may also generate meniscal tears $^{273,276}$. The presence of meniscal tears following ACL lesions has been found to significantly increase as time after initial injury increases ${ }^{276,277}$. Clinical studies of meniscal tears following ACL rupture indicate chronic damage to the medial meniscus more often than the lateral meniscus ${ }^{243}$. However, clinical studies of acute damage following ACL rupture are not consistent in the literature with some showing more lateral damage acutely ${ }^{245}, 247,248$, and some showing equality between medial and lateral meniscal damage $e^{243,244,246}$.

During normal human gait, the posterior third of the medial meniscus inhibits external rotation of the tibia, but excessive tension in the posterior horn is observed during repetitive impingement of the meniscus against the postero-medial femoral condyle ${ }^{274}$. This increased tension sometimes leads to tearing of the meniscus. Regional variations in patterns of meniscal damage have also been previously observed for patients with either stable or ACL-deficient knees ${ }^{278-280}$ ), including athletic populations ${ }^{275,281}$. There have been extensive investigations of the role the medial meniscus plays in the ACL-deficient $\mathrm{knee}^{280,281}$. However, isolated meniscal tears may be initiated with knee sprains and trauma, with or without the presence of ACL rupture ${ }^{272,273,279,281}$. Whether damage to the 
meniscus occurs at the time of trauma or sometime thereafter is not clear, and the mechanics of knee joint loading are imperative to understand for treatment and recovery after injury.

It is likely that trauma type influences the classification of meniscal tearing ${ }^{278}$. Bucket handle tears have been more frequently observed in skiers, basketball players, and soccer players, and have been very rarely observed in gymnasts and tennis players ${ }^{281}$. Reports of frequency and distribution of tears in medial and lateral menisci are difficult to analyze, as intrinsic and extrinsic factors likely influence the mechanism of injury, and thus the distribution of tears in specific populations. Although some researchers have reported a significantly higher incidence in tears of the medial meniscus for young athletes involved in sports such as skiing, basketball, and soccer, the frequency of tears between medial and lateral meniscal tears is nearly equal for gymnasts and volleyball players ${ }^{281}$. Whether or not athletes with acute ACL injury have predominance for tears of the medial meniscus over the lateral meniscus is not well understood ${ }^{244,281}$. Therefore, an understanding of meniscal injury mechanisms, as well as frequency, location, severity of meniscal tears, and involvement/injury of other structures in the knee, is imperative.

The motivation for this study was to explore spatial and temporal distribution of meniscal tears in a novel in vivo lapine model. This specific model mimics a high-energy jumplanding insult on the knee without bone fracture which results in ACL rupture. Previous investigations in the OBL have demonstrated that tibial-constrained impaction does not lead to meniscal tears ${ }^{282}$. Therefore, we investigated the trends of meniscal tears in rabbits without tibial constraint as well as in rabbits subjected to anterior cruciate ligament transection (ACLT). It was hypothesized that damage to the meniscus would differ between traumatically torn ACL and ACLT animals. 


\section{Materials and Methods}

\section{Animal Models}

Skeletally mature Flemish Giant rabbits $(5.4 \pm 0.3 \mathrm{~kg})$ were used in the study. The investigation was approved by the Michigan State University Institutional Animal Care and Use Committee. All animals were housed in individual cages (152 x 152 x $\left.36 \mathrm{~cm}^{3}\right)$ for the duration of the study. Eight rabbits received a blunt force insult to the left tibiofemoral (TF) joint using a previously described drop tower ${ }^{282}$ without tibial constraint. An additional three animals underwent unilateral surgical transection of the ACL in the left knee (ACLT). Five healthy, uninjured rabbits were used as age-matched morphological controls for both 12-week groups.

Animals undergoing blunt force insult to the $\mathrm{TF}$ joint were placed under general anesthesia (2\% Isoflurane and Oxygen). Following a previously described impact procedure (24), a $1.75 \mathrm{~kg}$ mass was dropped from a height of $75 \mathrm{~cm}(\sim 13 \mathrm{~J}$ of potential energy) striking the distal femur of the left leg. The sled was arrested electronically after one impact. A pre-crushed, deformable impact head (Hexcel, 3.76 MPa crush strength) was used to ensure uniform loading over the femur. The impact interface was mounted in front of a $4.45 \mathrm{kN}$ load transducer (model AL311CV, Sensotec, Columbus, OH). Prior to impact, the left limb was shaved. With the animal lying supine in the fixture, the knee was flexed $90^{\circ}$ and the foot fixed in a custom designed boot with three Velcro straps. An additional Velcro strap was crossed over the femur (Figure 5-1). The tibia was not constrained so as to allow for anterior subluxation of the tibia resulting in ACL rupture (TEAR group). Five animals were sacrificed immediately following traumatic ACL rupture. The remaining three animals received buprenorphine $(0.3 \mathrm{ml} / \mathrm{kg} \mathrm{BW})$ every 8 hours for 72 hours for post-trauma pain, and were sacrificed 12 weeks following impact. The right limb served as a non-impacted, contra-lateral control. Rupture of the ACL was characterized by joint laxity and verified using magnetic resonance imaging the day following impaction. 
Three additional animals underwent ACLT (LMD). Both rear legs of each animal were shaved from hock to the hip. The area was prepared using $70 \%$ betadine scrub and $70 \%$ alcohol, alternatively. Once scrubbed the rabbit was moved to a sterile surgery suite where, under sterile conditions, the left knee joint was exposed through a medial parapatellar arthrotomy. The patella was dislocated laterally exposing the ACL. With the knee in full flexion the ACL was transected. The joint capsule was sutured immediately after transection using 3/0 PDS. The sub-cutaneous layer and skin was closed in sequence using 4/0 PDS and staples, respectively. A sham operation was performed on the right limb in a similar fashion. Rabbits were monitored closely by a licensed veterinary technician [JA] for signs of pain. Post-surgery pain medication (Buprenorphine $0.3 \mathrm{ml} / \mathrm{kg}$ BW) was administered every 8 hours for 72 hours following the procedure. ACLT animals were sacrificed 12 weeks following surgery.

\section{Morphological Analysis}

The surfaces of the medial and lateral menisci of both limbs for all rabbits were stained with India ink to highlight surface fissures, tears, meniscal degeneration, and other irregularities. The surfaces were digitally photographed (Polaroid DMC2, Polaroid Corp., Waltham, MA) under a dissecting microscope at 12X and 25X (Wild TYP 374590, Heerbrugg, Switzerland). Gross morphological assessments were made according to the following criteria after the application of India ink: $0=$ normal; $1=$ fibrillar surface;

$2=$ undisplaced tears; $3=$ displaced tears ${ }^{283}$. Menisci were also assessed for tear type and location using previously established diagnoses ${ }^{284}$. Meniscal tears were also blindly classified by a Board Certified orthopaedic surgeon (DL).

\section{Histological Analysis}

For control $(n=3), 12$-week ACLT $(n=3)$ and 12-week TEAR $(n=2)$ rabbits, the left and right menisci of both limbs were freed of ligamentous attachments and synovium. Immediately after removal, menisci were rinsed in PBS and then fixed in 10\% formalin. Whole menisci bodies were then fixed in O.C.T.-30\% sucrose blend (TissueTek, Redding, CA, USA) for at least 48 hours. Six (6) $\mu \mathrm{m}$ cryosections were obtained and 
fixed on gelatin-magnesium sulfate coated slides. Slides were then subsequently stained using Fast Green-Safranin O (FG-SafO) staining for cellular morphology. Briefly, FGSaf $O$ results in mucins and cytoplasms stained blue-green, nuclei stained black, and sulfated glycosaminoglycans (GAG) stained red. Sections were then imaged using an Olympus AX70 Microscope and DP70 camera (Olympus Inc., Center Valley, PA, USA).

\section{Results}

\section{Gross Pathology and Grading of Meniscus}

Left limbs of all TEAR animals were positive for ACL rupture after insult. For all animals, the medial and lateral collateral ligaments and posterior cruciate ligament were intact in the left knee at time of sacrifice. All ligamentous structures were intact in the right uninjured limb.

Gross morphological assessments of the left limb menisci for acute TEAR, 12-week TEAR, and 12-week ACLT groups are illustrated in Figure 5-2. Left lateral menisci of all acute TEAR animals experienced distinct undisplaced tears (morphological score of 2 for all acute TEAR lateral menisci). Only one acute TEAR animal also experienced a medial meniscal undisplaced tear (Figure 5-2, Panel A). The four remaining medial menisci of acute TEAR animals were not visibly damaged. All left impacted limbs of animals in the 12-week TEAR group experienced gross morphological scoring of 3 for both medial and lateral menisci, indicated by displaced tears and loose tissue flaps, degeneration, and/or loss of menisci (Figure 5-2, Panel A). For the 12-week ACLT group, two of the three animals experienced gross morphological changes. Medial menisci of two ACLT animals experienced meniscal folding and displaced tears, degeneration, and severe bucket-handle tearing (morphological score of 3 for both animals). The lateral meniscus of one ACLT animal that experienced medial meniscal damage also showed severe degeneration, warranting a morphological score of 3 (Figure 5-2, Panel A). The third ACLT animal lacked gross morphological changes to either lateral or medial meniscus. 
For animals in the acute TEAR group, isolated lateral meniscal tears $(n=4)$ were more common than isolated medial meniscal tears $(n=0)$. Tear type was diagnosed for two acute TEAR animals. For one acute TEAR animal, a longitudinal tear was located in the red-red (RR) zone of the posterior region of the lateral meniscus. This rabbit also had a parrot beak tear in the red-white (RW) zone of the anterior region of the medial meniscus (Figure 5-2, Panel A). Another acute TEAR animal experienced a central region, whitewhite (WW) zone longitudinal tear of the lateral meniscus, which extended through the RR zone, with no gross medial meniscal damage.

For the left impacted limbs of all three animals in the 12-week TEAR group, tears were present in both lateral and medial menisci. For the lateral meniscus, one animal experienced a longitudinal tear similar to the longitudinal tears of the acutely injured animal. This animal also experienced a radial tear in the central/posterior region through the RW/RR zones in the lateral meniscus (Figure 5-2, Panel A). One of the animals experienced a WW parrot beak tear in conjunction with a longitudinal tear in the RW zone of the lateral meniscus. The third rabbit experienced lateral meniscal degeneration in the central and posterior regions. Degeneration of the medial meniscus in the central and posterior regions was observed in all three animals (Figure 5-2, Panel A).

All right limbs of TEAR and ACLT animals, except one, grossly appeared healthy, did not experience synovitis, and did not experience meniscal tears. The one animal that did have damage to the right limb was from the 12-week TEAR group. This animal demonstrated a lateral meniscus radial tear of the WW zone of the central region and slight degradation of the anterior region of the medial meniscus.

\section{Histopathology}

Differences in morphology between control, 12-week TEAR, and ACLT menisci were observed at the microscopic level. Representative cross-sections of control, 12-week TEAR, and ACLT left-limb menisci are illustrated in Figure 5-3. Positive staining for sulfated GAGs appeared to be equally distributed in RW and WW zones for central regions of control animals (Figure 5-3A). However, inconsistent coverage of sulfated 
GAGs was observed in the central regions for lateral TEAR and medial ACLT menisci (Figure 5-3E and I). A distinct longitudinal tear is observed in the mid-body region of a lateral TEAR meniscus (Figure 5-3E). Fibrillation of the medial ACLT menisci is also highlighted in Figure 5-3I (inset), which was observed in two of the three ACLT medial menisci. Fibrillation was also observed along the superior surface of lateral menisci for both 12-week TEAR animals. No microscopic fibrillation was observed in any control animals. Compared to healthy, control menisci, both medial and lateral menisci for TEAR animals demonstrated a high proliferation of cells at the synovium-meniscus junction (Figure 5-3F), as well as along the periphery of both menisci on deep and superior surfaces (Figure 5-3G). Cell proliferation at the synovium-meniscus junction for ACLT menisci was predominantly demonstrated in medial menisci (Figure 5-3J), however, both medial and lateral menisci of ACLT animals experienced superior and deep cellular proliferation (Figure 5-3K). Cell clustering and chondrocyte cloning was observed in both medial and lateral menisci for TEAR animals (Figure 5-3H). Cellular debris was observed in both ACLT medial and lateral menisci (Figure 5-3L).

\section{Discussion}

To our knowledge, this was the first in vivo injury model that replicates both acute and chronic meniscal damage following ACL rupture. This model imposed a specific and known mechanical insult on the tibiofemoral joint at the time of injury. This controlled mechanical environment promoted disruption of specific soft tissue structures of the knee, namely the menisci and ACL. Because the foot was constrained during impaction, it was unlikely that extensive tibial torsion was present at the time of ACL rupture. Nonetheless, damage to the meniscus was apparent immediately following this injury. This injury model also provided insight into possible mechanisms of meniscal injury. Acute meniscal lesions in lateral menisci were observed following dissection immediately after impaction with traumatic ACL tear. Extended damage to the menisci, particularly of the medial meniscus, progressed during several weeks following ACL rupture, likely accelerated by abnormal joint loading and stability. Lateral meniscal tears were more frequently observed in certain acute knee injuries where the knee undergoes 
torsional motion, perhaps because the lateral meniscus had more mobility within the joint and thus had greater exposure to unusual compressive and shear stresses ${ }^{243,} 281$. The controlled environment of this model mimicked that observed in knee injuries initiated by jump-landing ground impacts in sports such as alpine skiing ${ }^{247,285,286}$. For example, Duncan et al assessed acute ACL tears in alpine skiers within three days of injury and found that $83 \%$ of meniscal tears occurred in the lateral meniscus, with only $17 \%$ in the medial meniscus ${ }^{247}$. Similarly, Paletta et al and Inhara et al found an increase in lateral meniscal tears compared to medial meniscal tears in skiers ${ }^{285,286}$. This is reflected in our acute observations of predominantly lateral meniscal tearing. In combined soft tissue injuries, lateral meniscal tears have been reported to significantly outnumber medial tears in conjunction with medial collateral ligament and ACL tears ${ }^{287}$. Following acute knee twisting injury in 66 patients, the most frequent injury in conjunction with acute ACL rupture were found to be lateral meniscal tears (72.7\%), which overwhelmingly outnumbered the frequency of medial meniscal tears in the same group $(10.6 \%)^{288}$. Bone bruising of the lateral compartment has also been more frequently observed than in the medial compartment in MR images of the knee within four weeks of ACL rupture ${ }^{289,290,}$ with posterior-lateral bone bruising demonstrated in nearly $80 \%$ of ACL tears observed using MRI ${ }^{291}$. Nonetheless, the distribution of medial to lateral tears in conjunction with ACL rupture throughout the clinical literature is not clear ${ }^{243}$, likely due to the unknown mechanism of injury initiation. The precise mechanism associated with ACL rupture likely corresponds to the degree and location of meniscal damage. In our particular animal model, the frequency and distribution of acute meniscal tears was consistent and repeatable.

The currently investigated model demonstrated the relationship between acute and chronic damage to the meniscus. This study may also support previous literature findings that meniscal tears are typically accompanied with ACL ruptures and can be initiated in contact sports $^{272}$. Upon rupture, the ACL no longer acts to constrain the anterior motion of the tibia, thus provoking higher stresses in the posterior region of the tibia. Similar mechanics are thought to exist following ACLT. In this study, animals with surgical 
transection of the ACL did not demonstrate meniscal damage similar to animals with traumatic ACL rupture. While both ACLT animals and TEAR animals showed severe synovitis after 12-weeks, the frequency of lateral damage in the ACLT animals was much lower than in 12-week TEAR animals. The tear types also differed. Lateral menisci in 12weeks TEAR animals appeared to have distinct tears, whereas ACLT animals showed fibrillation and degeneration without any tear distinction. Conversely, medial menisci in TEAR animals after 12 weeks appeared to be degenerative and fibrillated, whereas medial menisci in the ACLT animals with apparent damage were marked by distinct meniscal displacement and bucket-handle tearing. The morphogenic differences between medial and lateral meniscal damage in these two injury models are noteworthy, as ACLT

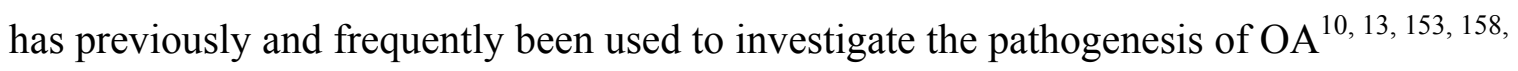
161,292

Previous investigations have found noticeable cell clustering and proliferation in the medial meniscus with minimal changes to the lateral meniscus eight weeks following $\mathrm{ACLT}^{10}$. Our study demonstrated a large amount of cell clustering and proliferation in both medial and lateral menisci twelve weeks following ACLT and in our novel traumatic injury model (TEAR). For both ACLT and TEAR animals, cell proliferation was increased throughout, and notably at the articulating surfaces and attachments with the synovium. Despite this similarity between the ACLT and TEAR animals, differences between the two injury models were also observed. In TEAR animals, both lateral and medial menisci experienced cellular clustering and cloning after twelve weeks, whereas in ACLT animals, a large amount of cell debris was present, perhaps due to necrosis of the fibrochondrocytes in the body of both menisci. Variations in the mechanical and biochemical environment of the knee may contribute to the microscopic differences between these two injury models. Future work investigating the inflammatory response following both ACLT and TEAR injuries should be pursued. Others have investigated the cellular and molecular response of the meniscus following ACLT. In contrast to the current study, Hashimoto et al also observed cell cloning in the medial menisci nine weeks following $\mathrm{ACLT}^{104}$. However, it is likely that cell clusters did exist in the current 
ACLT study, but were phagocytized after twelve weeks, as cellular debris was observed in all ACLT menisci. Cell cloning observed in TEAR menisci was noteworthy as it indicated a cellular response to injury similar to that of ACLT but perhaps at a different rate. The ACLT model may accelerate an inflammatory response caused by surgical incision that may lead to altered pathological changes compared to the TEAR model, which exhibited a solely impaction-induced cascade of matrix disruption, inflammation, and altered cell behavior.

Although the ACLT model induces joint instability, Mansour et al have suggested that altered joint kinematics might not be a critical factor in the development of $\mathrm{OA}^{293}$. Conversely, others have reported increased degeneration of the meniscus in ACLT animals over time ${ }^{10,158,283}$. Meniscal damage in clinical cases may be initially influenced by dynamic impaction events that contribute to bone, cartilage, and ligamentous damage. Also, it is likely that surgical procedures, such as arthroscopy and ACLT, influence synovial swelling, upregulation of inflammation, and pain, which also contribute to inhibition of mobility ${ }^{294}$. The chronic advancement of medial meniscal degradation several weeks following ACLT and traumatic ACL tear was apparent in this study. The development of the current model could potentially play a large role in investigating the implications of meniscal trauma in the progression of chronic joint disease.

Chronic overloading of the medial meniscus was likely responsible for advanced degradation of this structure following both ACLT and traumatic ACL rupture. Complex medial meniscal damage near the posterior horns has been reported to be more common in chronic ACL-deficient knees ${ }^{295}$. Using a finite-element model, Bendjaballah et al demonstrated increased medial loading in the ACL-deficient knee ${ }^{296}$. In a kinematic study, Waite et al observed greater medial tibial displacement and rotation in ACLdeficient knees throughout the stance phase ${ }^{297}$. The incidence of medial meniscal tears reported in previous literature has been demonstrated to increase with increasing time following ACL rupture ${ }^{243,277,298}$. In a ten-year follow-up study on sports-related injuries, nearly $24 \%$ of injuries to the knee involved the medial meniscus ${ }^{299}$. In this study, medial 
meniscal damage was more severe than lateral meniscal damage in the ACLT knee. Similarly, the low occurrence of medial meniscal tears at the time of traumatic ACL rupture in the current study and the prevalence of medial tears and degradation several weeks following injury may be a product of degeneration caused by chronic abnormal loading over time. Changes in cell behavior, observed in both injury models in the present study, were demonstrated by increased cellular proliferation and clustering, especially on peripheral boundaries of the meniscus. Such cellular changes may contribute to matrix alterations, perhaps attributing to the degeneration and reduced structural integrity following injury.

The locations of acute (lateral meniscus) and chronic (medial meniscus) tears in conjunction with traumatic ACL rupture in the present study are also, however, somewhat contrary to what has been observed in the literature. In a retrospective, multicenter study, nearly $37 \%$ of ACL ruptures were accompanied by medial meniscal tears, $16 \%$ had only lateral meniscal tears, and over $20 \%$ had both medial and lateral tears ${ }^{276}$. However, Cerabona et al investigated the frequency of meniscal tears in patients with acute ACL damage and found near equal distribution of tears in the medial and lateral meniscus $^{244}$. In our study, the frequency of tears was comparable between medial and lateral menisci for animals sustaining injury for twelve weeks, but not for animals immediately following injury. Previous investigations reflect injuries initiated in complex biomechanical environments of the knee, and the exact mechanism of ACL ruptures and meniscal tears was unknown. Our study represented an isolated compressive injury with known biomechanical parameters, thus providing a controlled environment useful for investigations of the etiology of meniscal degradation. 


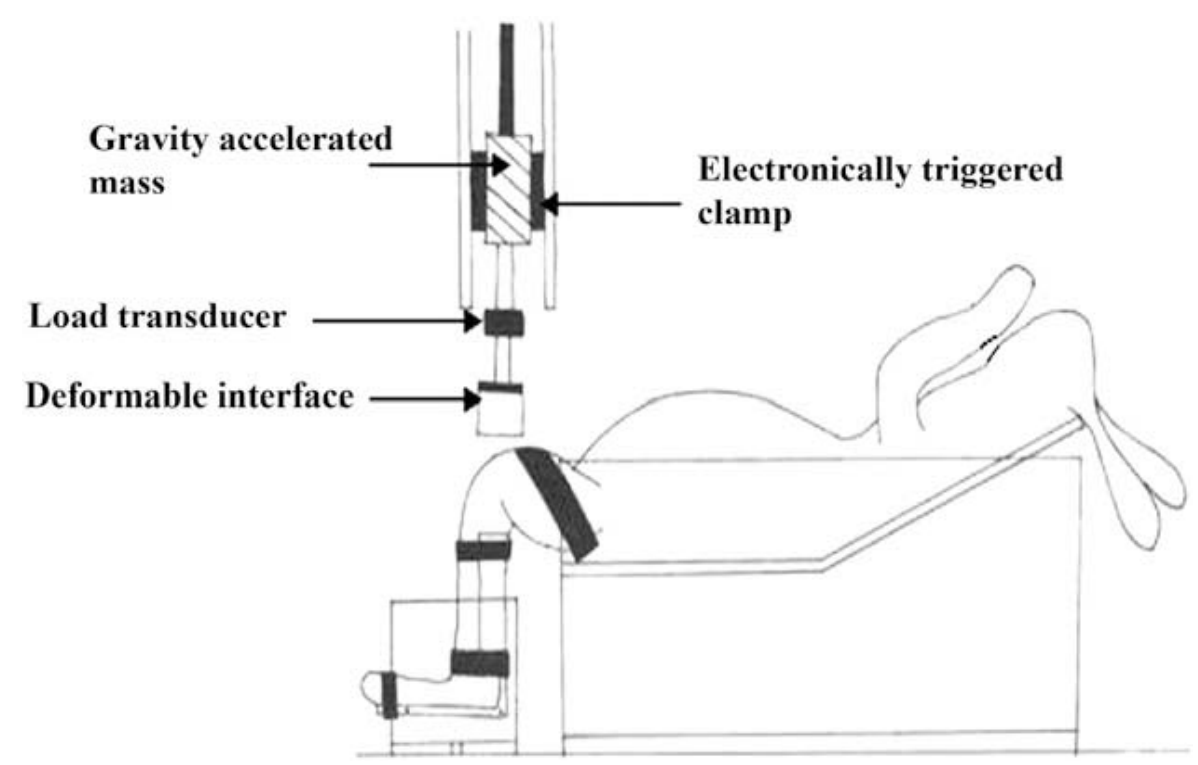

Figure 5-1. Schematic of traumatic impaction. Impact experiments were performed by dropping a gravity-accelerated mass onto the flexed tibial-femoral joint with approximately $13 \mathrm{~J}$ of potential energy. The rabbit was oriented such that the deformable interface struck the distal femur with impact forces oriented axially in the tibia.
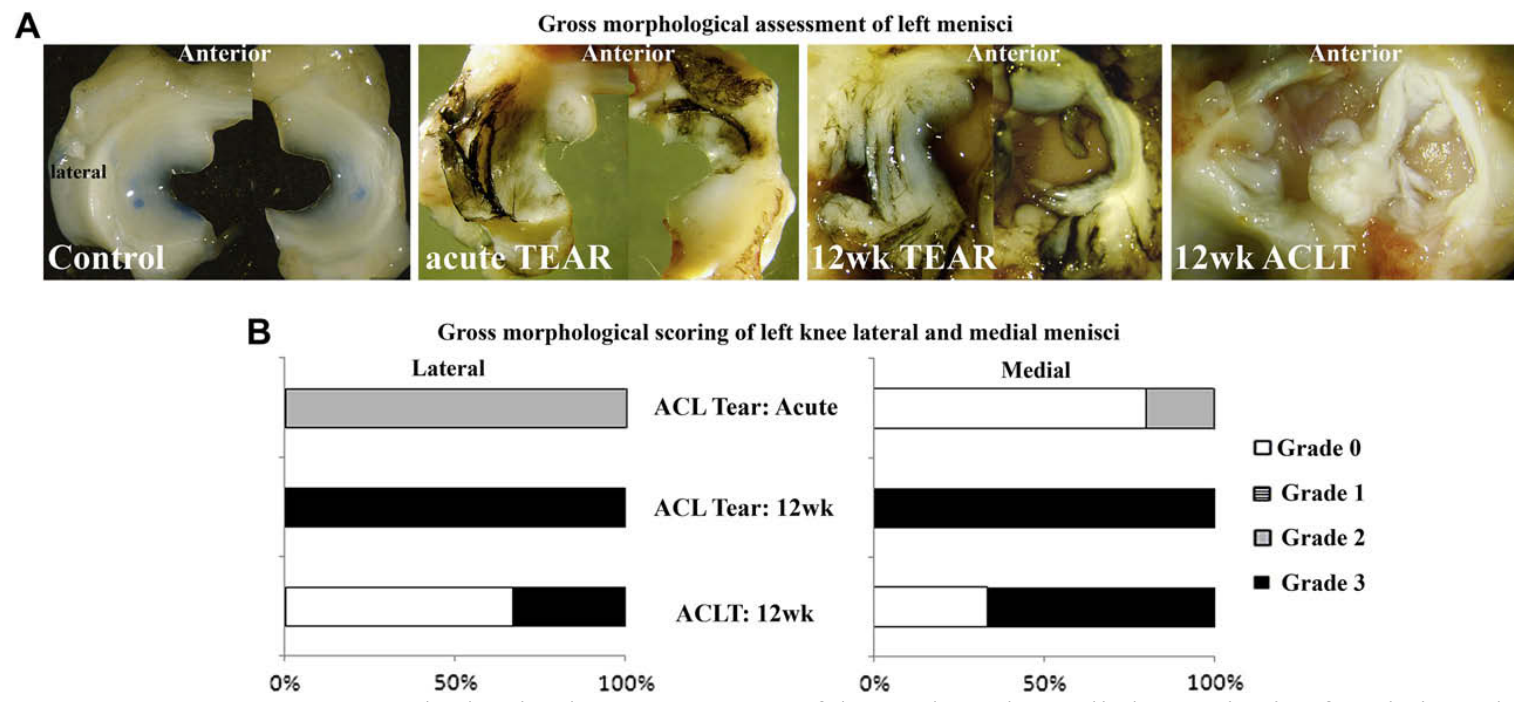

Figure 5-2. Gross morphological assessment of lateral and medial menisci of uninjured, acute TEAR, 12 wk TEAR, and 12 wk ACLT animals. (A) Gross morphological appearance of the lateral and medial menisci, from left to right, of each representative animal from uninjured, acute TEAR, 12 wk TEAR, and 12 wk ACLT groups. Note the longitudinal tears of the acute TEAR menisci, the progressed tearing and degeneration of the 12 wk TEAR menisci, and synovial swelling of the 12 wk ACLT menisci. (B) Scoring of the gross morphological changes overtime assessed by India ink staining. 

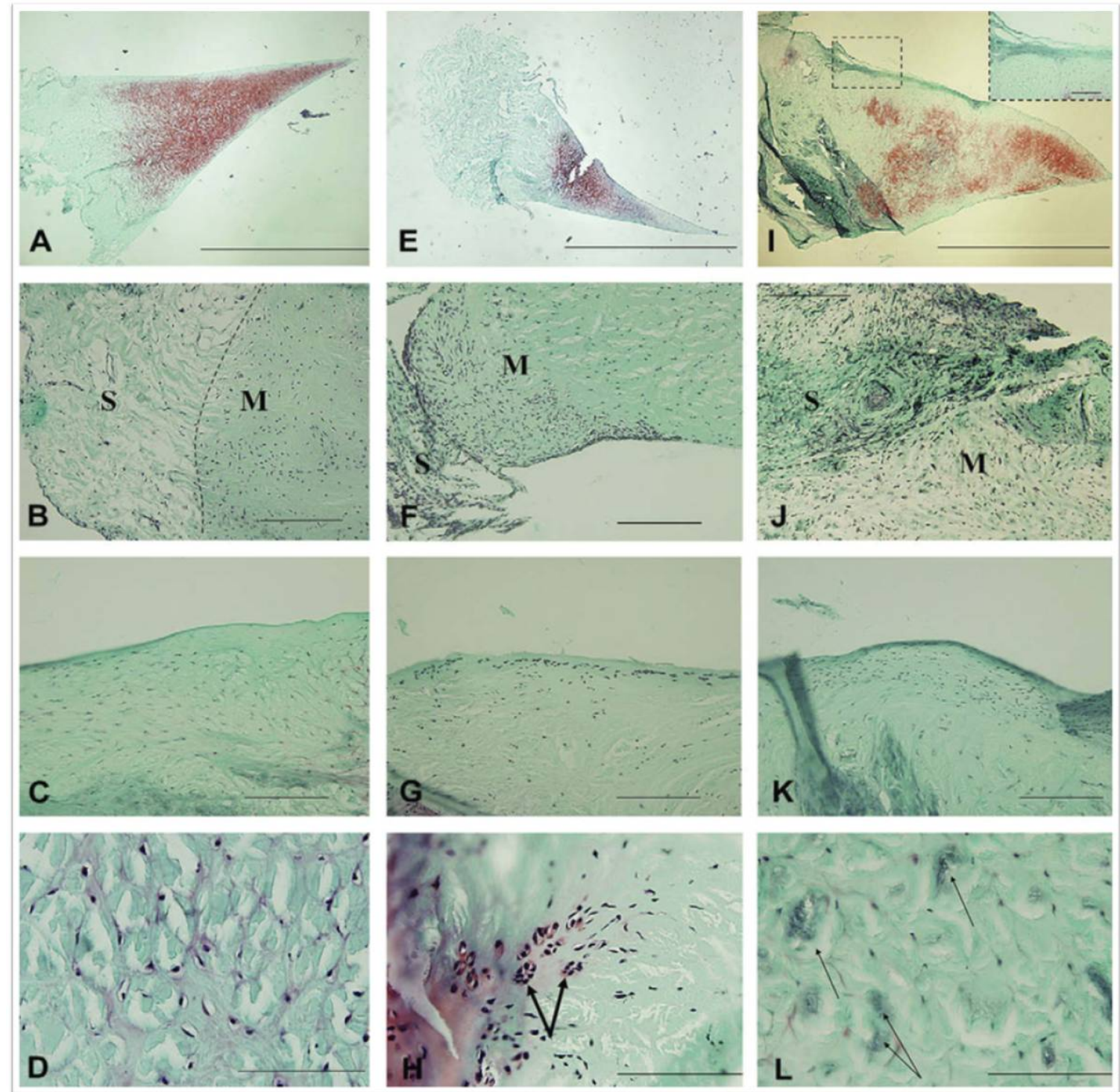

Figure 5-3. Microscopic structural and cellular findings of control (A-D), 12 wk TEAR (E-H), and 12 wk ACLT (I-L) left menisci. Coronal cross-section (A, E, and I) stained with FG-SafO illustrate intact control menisci with even distribution of sulfated GAG through RW and WW zones, a RW zone longitudinal tear in lateral meniscus of TEAR animal, and fibrillation of articulating surface of medial meniscus of ACLT animal [scale bar $=2 \mathrm{~mm}$ for A, E, and I]; synovial and meniscal junction (B, F, and J) illustrating high proliferation of cells for TEAR (F) and ACLT (J). Meniscus and synovium indicated with $\mathrm{M}$ and S, respectively. Articulating surfaces $(\mathrm{C}, \mathrm{G}$, and $\mathrm{K}$ ) for both TEAR and ACLT compared to control [scale bar $=200 \mu \mathrm{mfor} \mathrm{B}, \mathrm{C}, \mathrm{F}, \mathrm{G}, \mathrm{J}$, and K]; Fibrochondrocyte distribution $(\mathrm{D}, \mathrm{H}$, and $\mathrm{L})$ in control menisci appears even, with noticeable cell clustering and cloning for TEAR menisci, indicated with thick arrows, and cell debris for ACLT menisci, indicated by narrow arrows [scale bar $=100 \mu \mathrm{m}$ for $\mathrm{D}, \mathrm{H}$, and L]. 


\section{Chapter 6 - Regional Changes in Cellular and Tissue Morphology of Menisci Following Traumatic Impaction and ACL Tear}

\section{Abstract}

Objective: Regional differences in the healthy menisci, in terms of proteoglycan and GAG concentration, cellular density, and tissue area have been previously established. However, the progression of degeneration on these morphological characteristics has only been minimally investigated. This study investigated the role of traumatic impaction with anterior cruciate ligament (ACL) rupture and compared these findings with the previously established ACL-transection (ACLT) model.

Methods: The distribution of glycosaminoglycans (GAG), cellular density, and tissue area in both medial and lateral menisci across anterior, central, and posterior regions at twelve weeks following traumatic impaction injury, as well as ACLT, were measured and compared to that of healthy, uninjured controls and sham operated animals.

Results: A depletion of GAG coverage in medial and lateral menisci of both injury groups was observed. Increased tissue area was observed for regions of ACLT menisci, and increased cellular density was observed for menisci from traumatically impacted knees. Additionally, both models appeared to demonstrate calcium deposition in the menisci, but at different magnitudes of severity.

Conclusion: This study suggests differences between trauma-induced and surgicallyinduced instability models of osteoarthritis. Such differences encourage further investigations in the clinical relevance of a translational closed-joint animal model for investigating traumatically-induced OA.

\section{Introduction}

The meniscus functions to maintain knee joint health by distributing load and protecting the underlying cartilage of the tibial plateau from concentrated stresses of the articulating femoral condyles ${ }^{300}$. Damage to the menisci, as observed following traumatic impaction 
to the knee joint resulting from jump-landing sport accidents and automobile accidents, involve high compressive loads and often occurs in conjunction with anterior cruciate ligament (ACL) rupture ${ }^{134,301}$. Such damage has been clinically reported to lead to an accelerated rate of osteoarthritis progression and disability ${ }^{302,303}$. Previous research has illustrated the role of high-energy impaction directly applied to cartilage and its implications on cell viability ${ }^{304}$. Unfortunately, the resiliency of the meniscus following traumatic impaction has been rarely investigated ${ }^{305}$. It is suggested, however, that the health and integrity of the meniscus following traumatic impaction plays a role in osteoarthritis $(\mathrm{OA})^{27,231,306,307}$. Certain characteristics of the menisci, including its cellular density, glycosaminoglycan (GAG) content, collagen distribution, and shape, influence the behavior of this load-bearing tissue $\mathrm{e}^{253}$.

To date, numerous in vivo animal models have been used to investigate chronic advancement of OA of the knee $153,154,156,158,164,308,309$. However, the majority of these models involve surgical transection of the ACL (ACLT) and/or surgically-induced tears or removal of the menisci to mimic clinically observed damage. Although considered the "gold standard" of OA models 12, 153-159, 161, 164, 308-310, ACLT models may not reflect clinically relevant changes to the knee with respect to the traumatically injured joint and may be troublesome when used as a translational animal model. For example, bone bruising has been reported in over $80 \%$ of acute ligament rupture cases ${ }^{311}$, yet current animal models that implement ACLT lack this mode of injury ${ }^{231,243,312-315}$. From a clinical standpoint, little empirical research has investigated the chronic implications that traumatic impaction and its subsequent damage may have in the development of OA. Therefore, the implementation of a new animal model exploring these characteristics may prove useful for future traumatically-induced OA research.

Regional differences in the healthy menisci, in terms of proteoglycan and GAG concentration, cellular density, and tissue area 36, 78, 316-318 have previously been documented and are suggested to be due to the non-uniform loads placed upon the menisci ${ }^{236,} 318$. Additionally, decreased GAG content, recently suggested to occur 
following traumatic impaction ${ }^{319}$, suggests changes in soft tissue health and behavior, including increased permeability ${ }^{320}$ and friction levels ${ }^{321}$. In a closed joint model, it has been shown that isolated impaction to the knee with tibial restraint results in high magnitudes of compressive stress on the tibial plateau and an influx of chondrocyte death at the superficial layer ${ }^{282}$. Additionally, we have shown that ACL rupture, a consequence of anterior tibial translation following high-energy impaction, results in both acute and chronic meniscal morphological damage in the rabbit $k_{n e} e^{235}$. Uneven loading on the surface of the menisci is exacerbated by injury and altered mechanics. This altered loading characteristic may be elucidated by changes in GAG coverage of the meniscal body, as suggested in previous studies ${ }^{235,322}$, as well as tissue adaptations related to cellular density and tissue area.

In the present study, the cellular and matrix resiliency of the meniscus following traumatic impaction and ACL rupture was investigated twelve weeks following injury. These findings were compared with menisci of knees subjected to ACLT following a similar timeline. We investigated the role of traumatic impaction with ACL rupture in the induction of the chronic sequelae of meniscal degradation, and such damage was hypothesized to be more severe than that of age-matched animals subjected to ACLT. To test our hypothesis, the distribution of GAG, cellular density, and tissue area in both medial and lateral menisci at twelve weeks following traumatic impaction injury, as well as ACLT, was investigated.

\section{Materials and Methods}

\section{Animal model}

Animal treatment was approved by the Michigan State University Institutional Animal Care and Use Committee. A total of eleven Flemish Giant rabbits were used in this study. Three rabbits were subjected to a novel tibial impaction device that promoted anterior tibial translation. Briefly, animals were anesthetized and their left leg fitted at $90^{\circ}$ flexion. A high-energy force (approximately 13J) was applied externally to the anterior distal femur by means of a dropped mass impactor, with impact oriented axially through the 
tibia without translational constraint (TEAR group). The device used to impact the animals' knees was designed to mimic jump landings, which promote ACL injury, while retaining posterior cruciate, medial collateral and lateral collateral ligaments. Performing the impaction without anterior translational constraint resulted in ACL tears for all three TEAR animals ${ }^{235}$. Rabbits were given appropriate post-injury care and ACL tears were verified by magnetic resonance imaging.

A second group of animals $(n=3)$ were subjected to ACLT as previously described ${ }^{235}$. Briefly, both rear legs of each animal were shaved from hock to the hip. The area was prepared using $70 \%$ betadine scrub and $70 \%$ alcohol, alternatively. Once scrubbed, the rabbit was moved to a sterile surgery suite where, under sterile conditions, the left knee joint was exposed through a medial parapatellar arthrotomy. The patella was dislocated laterally exposing the ACL. With the knee in full flexion, the ACL was transected. The joint capsule was sutured immediately after transection using 3/0 PDS. The subcutaneous layer and skin was closed in sequence using 4/0 PDS and staples, respectively. A sham operation was performed on the right limb in a similar fashion, but the ACL was not transected.

All animals were monitored closely by a licensed veterinary technician for signs of pain. Post-surgery pain medication (Buprenorphine $0.3 \mathrm{ml} / \mathrm{kg}$ BW) was administered every 8 hours for 72 hours following the procedure. All animals were allowed normal cage activity. TEAR and ACLT animals were sacrificed at 12 weeks following injury. Another group of animals was maintained as normal, age-matched uninjured controls $(n=5)$.

Immediately after sacrifice, the knees of animals were carefully dissected and articular capsule opened. Lateral (L) and medial (M) menisci from both limbs were removed, immediately embedded in formalin and stored at $-80^{\circ} \mathrm{C}$. For histological staining, menisci were cut into anterior, central and posterior (A, C, P) regional sections (Figure 4-1) and embedded in Optimal Cutting Temperature embedding compound. Six micron $(6 \mu \mathrm{m})$ cryosections were obtained on gelatin-magnesium coated slides and subsequently stained. 
Slides were stained pair-wise ( $\mathrm{L}$ and $\mathrm{R}$ limb of each animal) for sulfated GAG coverage, calcium deposition, tissue area, and cellular density.

\section{Histomorphometry}

Paired slides were stained using Weigert's hematoxylin/Safranin O (FGSaf) for sulfated GAG coverage and Alizarin red for calcium deposition. Sections were imaged with a light microscope and digital camera. Quantifiable GAG coverage, illustrated by the amount of GAG-positive (red) staining on each sample, was determined using the R-G-B function with ImageJ software. The entire cross-section was calculated using area measure and the percentage of red-positive staining was then measured.

Histomorphometric scoring was performed by a blinded Board Certified pathologist. A newly established scoring system was developed to classify the activation of the synovial layer of the menisci, the morphology of the meniscal cells, and the quality of GAG staining (Table 6-1). A system to systematically grade microscopic damage of the menisci was developed based on histomorphometry scores of synovial layer, cell morphology, and quality of GAG (Table 6.2).

Alizarin red staining was used to identify calcium deposition in meniscal sections ${ }^{44}$. After staining, sections were graded on a scale of 0 to 4 by an independent observer, based on the scoring system previously established by Sun et $\mathrm{al}^{324}$ (Table 6.3).

Cells were detected using a propidium iodide staining technique and photographed with an Olympus AX70 microscope and DP70 camera. In brief, slides were placed in Coplin jars and fixed using $4 \%$ paraformaldehyde (PFA). Slides were then washed using PBS (pH 7.4), sections were covered by a proteinase $\mathrm{K}$ dilution $(10 \mathrm{mg} / \mathrm{ml})$ and re-fixed in $4 \%$ PFA. Slides were then immersed in a propidium iodide solution $(1 \mu \mathrm{g} / \mathrm{ml}$ in PBS) to stain the cell nuclei. Each section was imaged using fluorescence microscopy. Image analysis using CellC (Tampere University of Technology, Tampere, Finland) was performed to determine total cell count using an empirically determined cluster size. MetaMorph 
imaging software (Molecular Imaging, Downington, PA, USA) was used to determine the tissue area of each region.

Table 6-1. Scoring system for assessment of histomorphometric changes in menisci, classifying synovial layer, cell morphology, and quality of GAG staining of meniscal sections.

\section{Synovial layer}

0 Normal synovial layer

1 Slightly reactive synovial layer [focally prominent single

1 layer of cells of the synovial lining]

Moderately reactive synovial layer [2-3 cell-deep layer of

$2 \quad$ synovial cells on meniscal surface]

Over-reactive synovial layer [thick layers of synovial cells

3 on meniscal surface]

Cell morphology

0 Normal

1 Focal clustering of cells and damaged matrix integrity

1 [small clusters of cells, sparingly across cross-section]

Extensive clustering and clearing [several clusters and/or

2 large clusters of cells, and/or small regions of hypocellularity]

3 Degeneration [debris, large regions of hypocellularity

3 (>20\% of area) or acellularity]

4 Sparse cellularity and severe degeneration [few cells,

$4 \quad$ large degenerative regions]

\section{Quality of GAG}

0 Normal

1 Thinning of GAG [reduced intensity and inconsistencies]

2 Redistribution and thinning of GAG [inconsistent GAG

2 coverage, migration of GAG to surface, GAG pockets]

3 Focal pockets of GAG [devoid of GAG except in distinct,

3 irregular, small regions] 
Table 6-2. Grading and characterization of microscopic meniscal damage.

\begin{tabular}{|lll|}
\hline Characterization & Score & Description \\
\hline Grade 0 & $0-2$ & Normal, healthy menisci \\
\hline Grade 1 & $>2-4$ & Mild damage \\
\hline Grade 2 & $>4-7$ & Moderate damage and degenerative changes \\
\hline Grade 3 & $>7$ & Severe damage and degeneration \\
\hline
\end{tabular}

Table 6-3. Alizarin red scoring criteria ${ }^{324}$ for menisci from healthy and injured limbs.

\begin{tabular}{|ll|}
\hline Score & \begin{tabular}{l} 
Criteria \\
\hline $0 \quad$
\end{tabular}$\quad \begin{array}{l}\text { no calcium deposition } \\
\text { edges of the meniscus }\end{array}$ \\
\hline 2 & $\begin{array}{l}\text { limited number of clusters of small-sized and medium-sized calcium deposits at } \\
\text { the edges of the meniscus }\end{array}$ \\
\hline $3 \quad \begin{array}{l}\text { clusters of small calcium deposits inside the meniscus and limited number of } \\
\text { clusters of small-sized and medium-sized calcium deposits at the edges of the } \\
\text { meniscus }\end{array}$ \\
\hline $\begin{array}{l}\text { clusters of small-sized calcium deposits inside the meniscus and widespread } \\
\text { clusters of medium-sized and large-sized calcium deposits at the edges of } \\
\text { meniscus }\end{array}$ \\
\hline
\end{tabular}

Cells were detected using a propidium iodide staining technique and photographed with an Olympus AX70 microscope and DP70 camera. In brief, slides were placed in Coplin jars and fixed using 4\% paraformaldehyde (PFA). Slides were then washed using PBS (pH 7.4), sections were covered by a proteinase $\mathrm{K}$ dilution $(10 \mathrm{mg} / \mathrm{ml})$ and re-fixed in $4 \%$ PFA. Slides were then immersed in a propidium iodide solution $(1 \mu \mathrm{g} / \mathrm{ml}$ in PBS) to stain the cell nuclei. Each section was imaged using fluorescence microscopy. Image analysis using CellC (Tampere University of Technology, Tampere, Finland) was performed to determine total cell count using an empirically determined cluster size. MetaMorph imaging software (Molecular Imaging, Downington, PA, USA) was used to determine the tissue area of each region. 


\section{Statistics}

Data are presented in the text as mean \pm standard error. A one-way ANOVA was used to determine statistically significant differences between animal groups within each region for each of the metrics investigated: GAG coverage, tissue area, and cell density. Additionally, F-tests were performed to determine equal or unequal variance between control, TEAR, and ACLT groups based on anatomical location. Post-hoc two-sample ttests with respective equal or unequal variance assumptions were used to isolate significant differences between groups $(\mathrm{p}<0.05)$. A one-way ANOVA was also used to determine differences between regions within each group.

\section{Results}

Average scores of histomorphometric damage from all regions of the meniscal sections varied between groups (Table 6-4). The ACLT group demonstrated the greatest amount of damage, characterized as moderate with degenerative changes in both medial and lateral menisci. The TEAR and ACLT sham groups demonstrated mild damage in both menisci (Table 6-4).

Table 6-4. Histomorphometric scoring of both medial (M) and lateral (L) meniscal sections for Control, ACLT, TEAR and ACLT sham groups.

\begin{tabular}{|l|lll|}
\hline Menisci & Group & Score & Grade \\
\hline \multirow{4}{*}{ M } & Control & 0.8 & Normal, healthy menisci \\
\cline { 2 - 4 } & ACLT & 4.5 & Moderate damage and degenerative changes \\
\cline { 2 - 4 } & TEAR & 3.4 & Mild damage \\
\cline { 2 - 4 } & ACLT Sham & 2.7 & Mild damage \\
\hline \multirow{4}{*}{$\mathbf{L}$} & Control & 0.1 & Normal, healthy menisci \\
\cline { 2 - 4 } & ACLT & 4.1 & Moderate damage and degenerative changes \\
\cline { 2 - 4 } & TEAR & 3.0 & Mild damage \\
\cline { 2 - 4 } & ACLT Sham & 2.0 & Mild damage \\
\hline
\end{tabular}


Morphological damage to the menisci was observed 12 weeks following both TEAR and ACLT (Figure 6.1). Similar trends were observed in the depletion of GAG coverage in medial and lateral menisci of both injury groups (Figure 6.1). For both ACLT and TEAR groups, GAG coverage decreased across all regions. However, this decrease was only significant for both groups in the MC and LC regions (Figure 6.1). Additionally, a decrease in GAG coverage was observed in the LA and LP regions for the ACLT group (Figure 6.1). Hypercellularity, cell clustering, and cell depletion, as well as GAG depletion, were also noted for both TEAR and ACLT groups from a qualitative standpoint (Figure 6.1). Both ACLT and ACLT sham groups demonstrated matrix degeneration across all regions, indicative of "blebbing", primarily in the outer zone of the tissue (Figure 6.1). This characteristic was not observed in any TEAR or control samples. Menisci from R limb of TEAR group did not qualitatively differ from menisci from control group (data not shown).

Calcification of the menisci was observed in both ACLT and TEAR groups (Figure 6-2; Table 6-5). In TEAR menisci, mild calcification was observed, with clusters at the edges as well as small depositions in the body of the menisci (Figure 6-2). In the ACLT group, the deposition of calcium was more profound than the TEAR group, and clusters appeared throughout the meniscal body (Figure 6-2). Large clusters of calcium deposition at the edges were observed in the ACLT (Figure 6-2E). The healthy, uninjured menisci did not demonstrate calcification (Figure 6-2). Additionally, menisci from unimpacted limbs of the TEAR group and from the Sham group did not demonstrate calcification (images not shown).

Table 6-5. Grade of alizarin red staining.

\begin{tabular}{l|cccc|} 
& Control & ACLT & TEAR & \multicolumn{1}{c}{$\begin{array}{c}\text { ACLT } \\
\text { Sham }\end{array}$} \\
\cline { 2 - 5 } Medial & 0.3 & $3.3 \pm 0.12$ & $1.9 \pm 0.02$ & $1.3 \pm 1.90$ \\
\cline { 2 - 5 } Lateral & 0 & $2.9 \pm 0.60$ & $2.4 \pm 0.30$ & $1.5 \pm 2.12$ \\
\cline { 2 - 5 } Average $^{*}$ & 0.2 & $3.1 \pm 0.24$ & $2.1 \pm 0.10$ & $1.4 \pm 1.96$ \\
* average of medial and lateral scores &
\end{tabular}


Healthy menisci appeared to have congruous, evenly distributed GAG coverage when present (Figure 6.1), along with evenly distributed cells (Figure 6-3i) and a normal synovial layer. The ACLT group appeared to be substantially more degenerative than the TEAR group from a morphometric standpoint. The ACLT group demonstrated focal pockets of GAG (Figure 6.1), degeneration and sparse cellularity (Figure 6-3iv), and an over-reactive synovial layer (Figure 6-3v). The TEAR group appeared to have a slight to moderately reactive synovial layer (Figure 6-3), redistribution and thinning of GAG (Figure 6.1), and focal-to-extensive clustering and cell clearing (Figure 6-3ii and iii).

In the menisci 12 weeks following ACLT, the average cross-sectional area across all regions was significantly increased $\left(8.4 \pm 1.0 \mathrm{~mm}^{2}\right)$ compared to that of the healthy control $\left(4.3 \pm 0.3 \mathrm{~mm}^{2}\right)$, the ACLT sham $\left(4.2 \pm 0.3 \mathrm{~mm}^{2}\right)$ and TEAR groups $\left(3.9 \pm 0.3 \mathrm{~mm}^{2}\right)$ (Figure 6-4). Regional differences in tissue area between groups were further investigated, illustrating a significant increase in tissue area for the ACLT group in the MA and LP regions compared to the control group (Figure 6-4). Interestingly, the TEAR group showed the opposite trend when compared to the control group, in particular with a significant decrease in tissue area in the LP region (Figure 6-4). These results indicated meniscal swelling following ACLT, which was not observed in the TEAR group.

Menisci of the TEAR and sham groups experienced a significant increase in average cell density $\left(559.8 \pm 36\right.$ cells $\left./ \mathrm{mm}^{2}\right)$ compared to the healthy control group $(435.3 \pm 23$ cells $/ \mathrm{mm}^{2}$ )(Figure 6-4), suggesting hypercellularity after 12 weeks of injury. The sham group only saw a significant increase in cellularity in the medial menisci (Figure 6-4). The ACLT group did not differ significantly from the control group in cell density $\left(488 \pm 39\right.$ cells $\left./ \mathrm{mm}^{2}\right)$. Differences in regional cell density were particularly influenced by injury in the lateral menisci. A significant increase in cell density was observed in the LA region for the TEAR group, whereas a decrease in cell density was found in the LP region for the ACLT group (Figure 6-4). 


\section{Discussion}

This study illustrated two distinct classifications of degeneration and OA progression in lapine menisci. The first classification, demonstrated in the TEAR group, includes the depletion and thinning of sulfated GAG and changes in cellularity. Specifically, this entailed the clustering of fibrochondrocytes, the activation of the synovial layer of the meniscus, and the focal depletion of cells. The second classification, demonstrated in the ACLT group, includes the degeneration of the meniscal matrix, indicative of cell-like blebs, the accumulation of calcium, and the depletion, thinning, and focal accumulation of GAG. Additionally, the ACLT group modeled an increased in tissue area, which suggests water retention ${ }^{323}$. This, in combination with a decrease in GAG coverage, has been previously identified as a model of advanced degeneration in the ACLT knee ${ }^{323}$. It is possible that these two models may demonstrate differing classifications of OA after 12 wks of damage. It is possible that these separate models replicate two different etiologies of OA; specifically, these models may represent primary and secondary OA in the ACLT and TEAR models, respectively. In the ACLT model, OA progresses during the $12 \mathrm{wks}$ post-surgery without the presence of acute compression-induced damage. Although the ACLT model leads to more accelerated joint degenerative changes than the TEAR model at similar timepoints, the end result is mimetic of clinically observed idiopathic $\mathrm{OA}^{324}$. In the TEAR model, the progression of OA is likely influenced by acute damage to the menisci as well as subchondral bone microcracks ${ }^{322}$. Albeit slower, the TEAR model may involve alternate mechanisms not observed in the ACLT model, such as post-traumatic healing and altered inflammatory signaling, that are observed in the post-traumatic knee. The etiology of OA in the ACLT model is assumed to be implicitly related to altered biomechanics, whereas the progression of degeneration to the knee in the TEAR model is likely more complex. Recently, McGonagle et al has suggested anatomical-based classifications for $\mathrm{OA}$ as opposed to the traditional classifications of primary (idiopathic) and secondary (eg. post-traumatic) derivations ${ }^{325}$. Based on the observed differences between the ACLT and TEAR models in meniscal 
degeneration, the present study encourages future investigations in meniscogenic mechanisms of osteoarthritic development.

Traumatic injury resulting in ACL rupture led to complex changes in the meniscal tissue, especially pertaining to GAG coverage, cellular density, and tissue area, and was compared to changes in meniscal tissue following ACLT. This is the first study to quantitatively explore changes in GAG coverage of the meniscus following both traumatic ACL rupture and ACLT, and this work provides insight into the short-term degradative pathway associated with both traumatic impaction and altered loading. It can be argued that, in the ACLT model, the loading environment is the only characteristic majorly altered in order to replicate arthritic-like changes to the knee joint. Such mimicry may not be reflective of changes that result from post-traumatic impaction and anterior tibial translation observed in the clinical setting. The ACLT model may be more

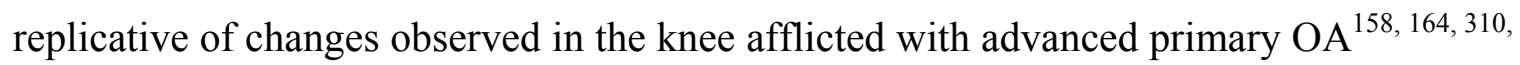
${ }^{326}$, in that it replicates advanced joint degeneration without a multifaceted acute injury. The TEAR model imposes a more complex pathway of damage, specifically: ACL rupture $^{235}$, bone microcracks ${ }^{322}$, and meniscal tears ${ }^{235}$. These model characteristics may relay subsequently different rates and characteristics of OA development than its preceding ACLT model. Thus, the TEAR model likely imposes a more clinically relevant model for investigating secondary, or traumatically-induced, OA.

The findings of this study suggest that impaction-induced trauma, which resulted in pathological loading, can influence the matrix of the menisci differently than pathological loading alone. Increased cellular density in regions of the TEAR group may suggest cellular proliferation of fibrochondrocytes and/or synoviocytes. Previous work by Hellio Le Graverand et al using the ACLT rabbit model have suggested that cell proliferation may contribute to the clustering and phenotypic changes of meniscal cells during the developmental stages of osteoarthritis ${ }^{159}$. However, it is not clear in this study if cell proliferation or migration is occurring. Additionally, it is unclear if increased cellular density alone is detrimental to the menisci. Some suggest increased proliferation may 
lead to increased vascularity, healing, and repair ${ }^{327,}{ }^{328}$. Nonetheless, differences in cellular density exist between the ACLT and TEAR groups, and this may also underline differences in the progression of OA between the two models.

The presence of meniscal calcification of OA patients has just recently brought to light the likely role of the meniscus in degenerative knee $\mathrm{OA}^{324}$. Previously, hyaline cartilage was thought to be primarily responsible for the production and secretion of calcium into the joint. However, Sun et al recently showed that not only is the menisci of OA patients calcified, meniscal cells from OA patients also deposit more calcium than healthy cells when isolated and cultured in vitro ${ }^{324}$. As such deposition persists, the secretion and release of calcium to the synovial fluid can be further damaging to the joint as a whole ${ }^{329}$. Thus, our findings of increased calcification in the ACLT menisci are important for investigating advanced-stage OA. Monitoring the progression of meniscal calcification, as well as GAG depletion and changes in cell morphology, may elucidate the exact role of the meniscus in idiopathic OA as well as traumatically-induced OA.

Our findings that chronic ACLT induces morphological changes in the menisci are not new. Previous literature by Sonoda et al explored the change in tissue area and GAG coverage of rabbit menisci, suggesting the role of meniscal swelling and increased cell proliferation 9weeks after ACLT in OA progression ${ }^{323}$. Additionally, changes in cell phenotype and the formation of clusters have been noted within four weeks following ACLT ${ }^{159}$. However, this is the first study of its kind to explore differences in such metrics between a new, closed joint traumatic injury animal model and the "gold standard" ACLT model. It is important to note that cell morphology changes, specifically cell clusters and cell islands, observed in the new TEAR model 12 weeks following surgery are similar to those seen by Hellio Le Graverand in the ACLT model after 4 weeks ${ }^{159}$. Cellular and matrix degeneration, present in histological assessment of ACLT specimens, may be a more advanced form of the observed cell cluster formations present in the TEAR specimens ${ }^{235}$. This, along with the deposition of calcium in the ACLT menisci, suggests that the ACLT model likely progresses more rapidly than the 
TEAR model. Such an accelerated advancement of OA-like changes in the ACLT meniscus may shrink the observational window for investigating treatment efficacy and disease progression. Likewise, meniscal swelling and accelerated morphological changes may dissuade researchers from understanding the natural progression of traumaticallyinduced meniscal damage. The present study underlines the need for further studies, with multiple time points, investigating the immediate and long-term biomolecular responses of the meniscus to impaction-induced trauma.

The qualitative assessment of cell clusters as well as cell depletion in injured meniscal cross sections investigated in this study provide some insight into pathological changes for both TEAR and ACLT models. Although the lateral menisci of the TEAR group did not appear to be GAG depleted from a quantitative standpoint, the reduced quality and lack of congruency in GAG coverage, as well as the deviation in cell morphology compared to the control group, were suggested from a qualitative standpoint. Previously, it has been well documented that even marginal changes in proteoglycan and GAG content influence the load-bearing properties of cartilage and meniscus ${ }^{80,330,331}$. In articular cartilage, decreased GAG coverage, present before other indicators of damage, has been suggested as an indicator of degenerative changes to the tissue's structure and stability ${ }^{332,333}$. Measurement of sulfated GAG coverage using Safranin O staining as described in the present study provides useful information in the degeneration and degradation patterns of the meniscus following injury from both a quantitative and qualitative standpoint.

Regional morphological and histological changes to the meniscus have not been previously investigated with a non-surgical (closed-joint) instability injury model. However, regions of the meniscus may experience changes in local stresses after the integrity of both ACL and menisci have been compromised. In fact, it has been suggested by their material and molecular makeup that anterior, central, and posterior regions of the menisci experience different loading parameters ${ }^{236}$. Loss of meniscal integrity leads to reduction in the contact area between the femoral condyles and tibial plateau, resulting in 
$50-70 \%$ higher stresses on the exposed articular cartilage ${ }^{334-336}$. It is noteworthy that the medial meniscus saw similar decreases in GAG coverage for the TEAR and ACLT groups when compared to the healthy rabbits in this study. Increased strain levels, as seen in the remaining meniscus following meniscectomy ${ }^{95}$, have been shown to influence the amount of GAG released from the meniscus in vitro ${ }^{174}$. Chronic decrease in GAG coverage for the injured groups in this study may suggest increases in strain levels on the remaining menisci; however, such changes can only as of now be speculated as it is difficult to measure in vivo strains on the lapine menisci.

The presence of meniscal tears, impaction-induced bone bruising, inflammation, and other soft-tissue damage that can be associated with trauma-induced ACL tears may influence the maintenance of GAG and cellular density in the knee ${ }^{235,289,337,338}$. In this study, differences between TEAR and ACLT models were apparent, particularly in cell density and tissue area. Acute meniscal tearing in TEAR animals was not present in the ACLT animals ${ }^{235}$. Therefore, this study suggests the combined influence of acute and chronic degenerative influence of trauma-induced instability. Recently, Meyer el at described the "footprint" of bone bruising in the subchondral bone that matched tibiofemoral contact pressures during compression-induced ACL rupture ${ }^{338}$. The nonsurgical OA animal model described in this study has been suggested to mimic sporting accidents, especially related to downhill skiing ${ }^{247}$. Although no gross fracture took place following impaction, it is likely that microfracture occurred in the subchondral tibiofemoral joints of the TEAR animals. Such microfracture and bone bruising, observed with TEAR but not ACLT, likely play a role in the natural development of OA.

In summary, this study used two different lapine models to investigate the progression of $\mathrm{OA}$ and damage to knee meniscus. While both models contributed to significant changes in the microscopic behavior of meniscal tissue, it is important to address the differences between these two models. Where the ACLT model demonstrated more accelerated degradation and matrix changes in the meniscus, the traumatic model closely replicates what is observed in the clinical setting ${ }^{247}$. Additionally, the traumatic model will provide 
insight into the clinically observed cases of joint mechanics alterations, bruising, and inflammation immediately following injury to the knee joint, as opposed to the ACLT model which specifically replicates altered joint mechanics alone. Therefore, this study provides rationale for future investigations using this novel model for investigations in non-surgical, closed-joint meniscal damage as well as OA.

\section{Recommendations}

The menisci are being further elucidated as playing a major role in the development and advancement of OA. By using the TEAR model, future studies can investigate how timedependent changes in the menisci evolve following traumatic ACL tear. It is important to investigate meniscal morphometric changes at various periods of time to determine critical time points for implementing post-injury treatment options. It may also be worthwhile to investigate the deposition of calcification of the menisci following ACLT and TEAR. Inhibiting the deposition of calcium in the menisci may develop future treatment options translatable to the clinic in order to preserve the integrity and mechanical function of the healthy menisci.

\section{Acknowledgements}

This study was supported by a grant from the Centers for Disease Control and Prevention, National Center for Injury Prevention and Control (CE000623). Its contents are the sole responsibility of the authors and do not necessarily represent the official views of the Centers for Disease Control and Prevention. Animal care and injury model development was performed at the Orthopaedic Biomechanics Laboratories at Michigan State University by Daniel Isaac and Dr. Eric Meyer under the supervision of Dr. Roger Haut. 


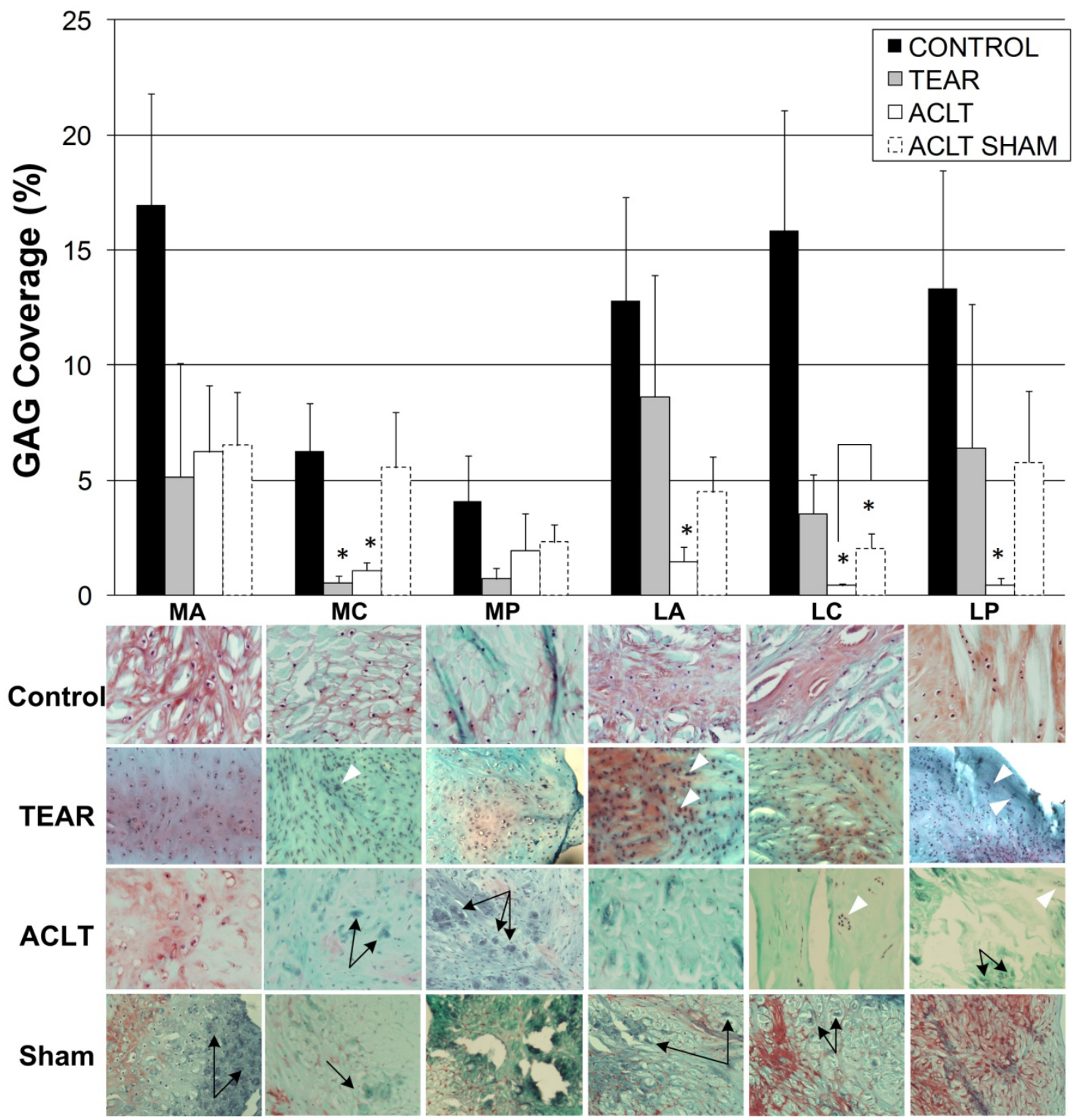

Figure 6-1. GAG coverage as a percentage of cross-sectional area [mean + SE] (top) and micro-morphology (bottom) of control, TEAR, ACLT, and sham ACLT menisci for medial $(\mathrm{M})$ and lateral $(\mathrm{L})$ anterior $(\mathrm{A})$, central $(\mathrm{C})$, and posterior $(\mathrm{P})$ regions. Note cell clusters (white arrow heads) as well as lack of cells in TEAR meniscal sections, as well as cell debris and cell clusters (black arrows) in ACLT and ACLT sham meniscal sections. Scale bars indicate $100 \mathrm{um} .{ }^{*}=$ significantly less GAG coverage than control group for same region. 


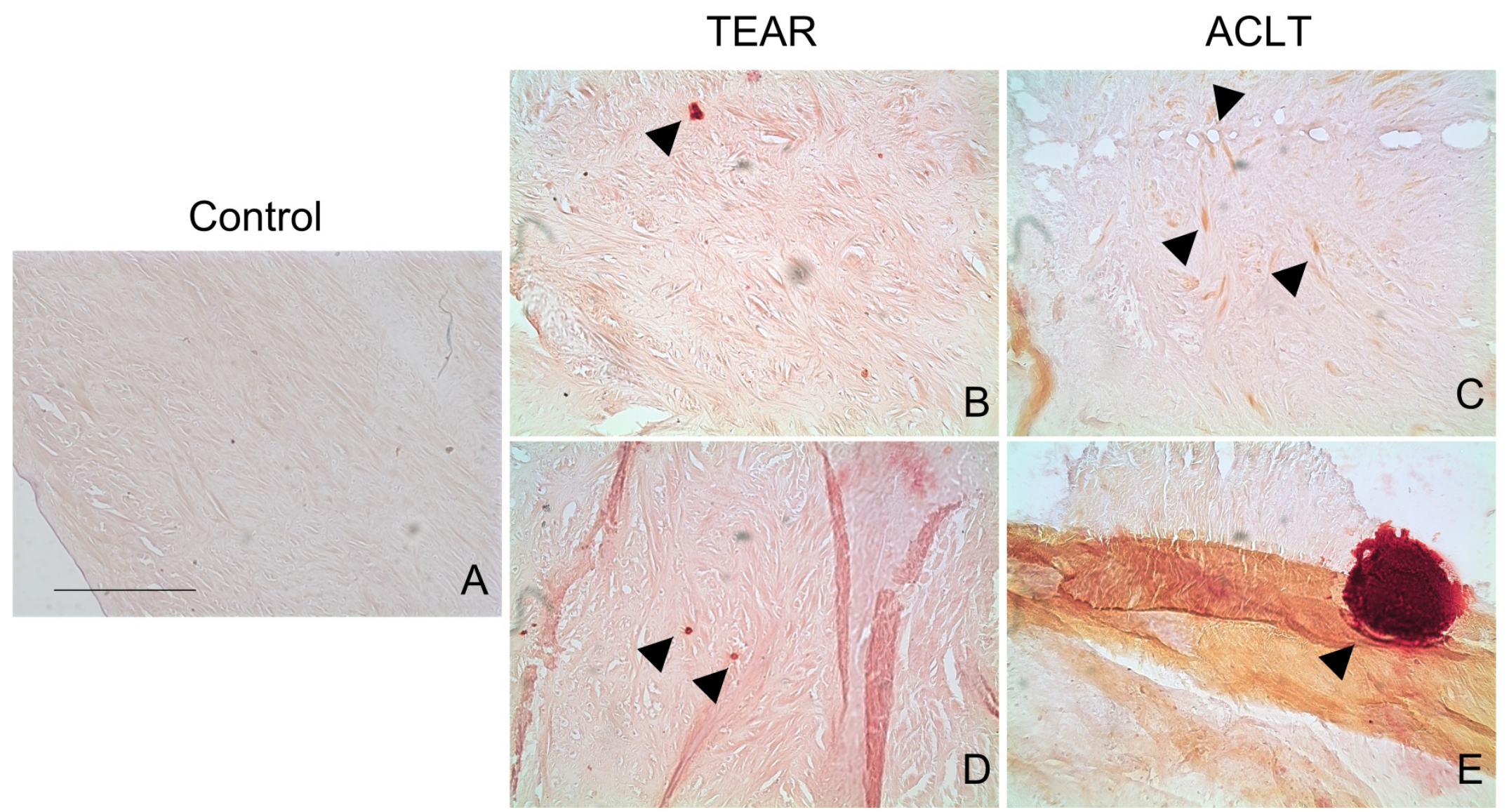

Figure 6-2. Menisci stained with alizarin red of control (A), TEAR (B \& D), and ACLT (C \& E) groups. There was no calcium deposition in control menisci (A). Black arrows indicate calcium depositions. Some regions of TEAR menisci demonstrated calcium deposition on the periphery and small clusters on the inside of the menisci (B and D). In the ACLT group, clusters of small calcium deposits were present on the inside of the menisci $(\mathrm{C})$ and larger clusters were present on the periphery $(\mathrm{E})$. Scale bar $=500 \mu \mathrm{m}$. 


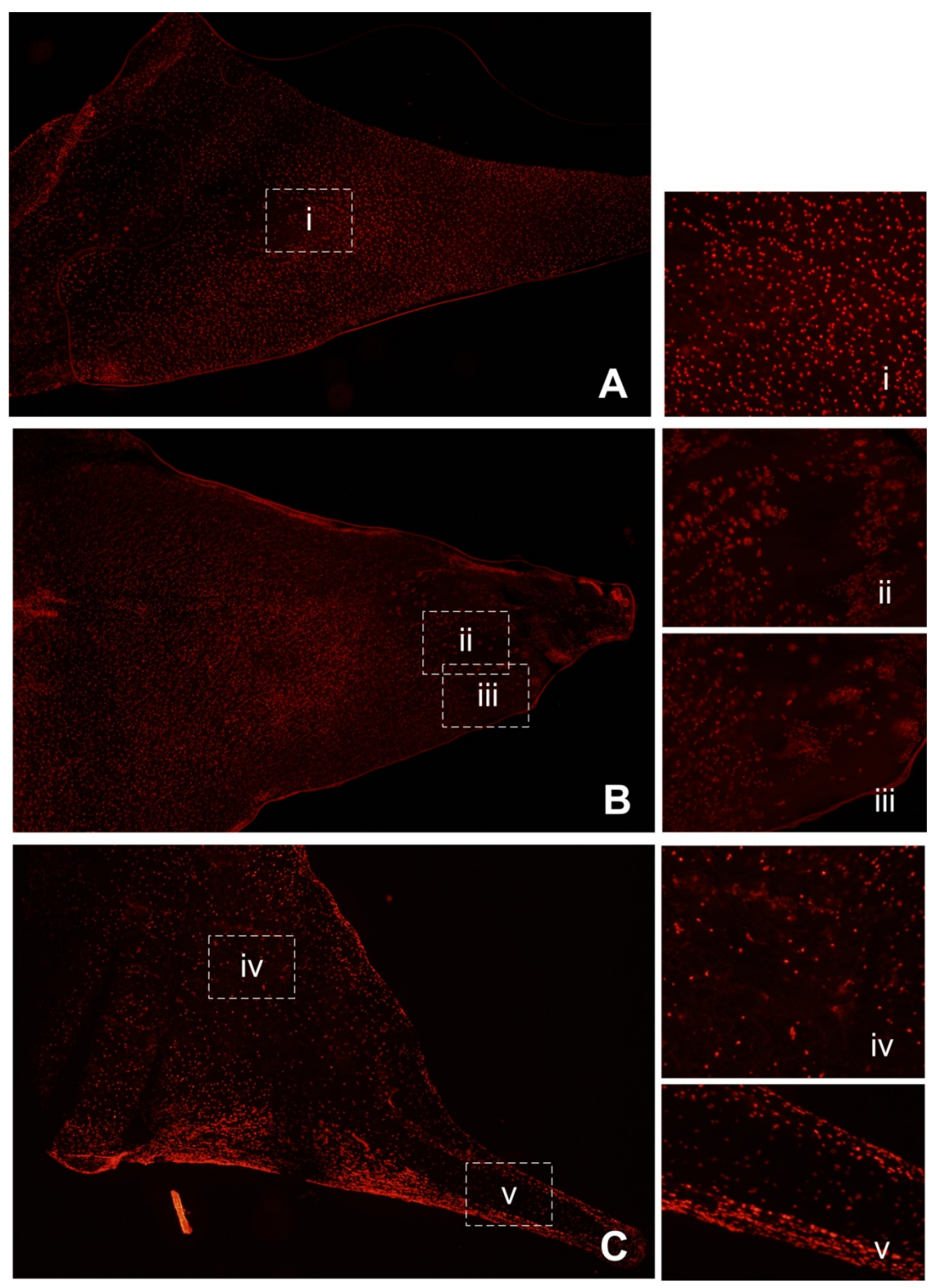

Figure 6-3. Cell morphology of healthy (A), TEAR (B), and ACLT (C) from the anterior region of the lateral menisci. Insets illustrate normal distribution of cells in the middle zones of healthy tissue (i), cell clustering and hypocellularity (ii and iii), sparse cellularity (iv) and over-reactive synovial lining (v). 

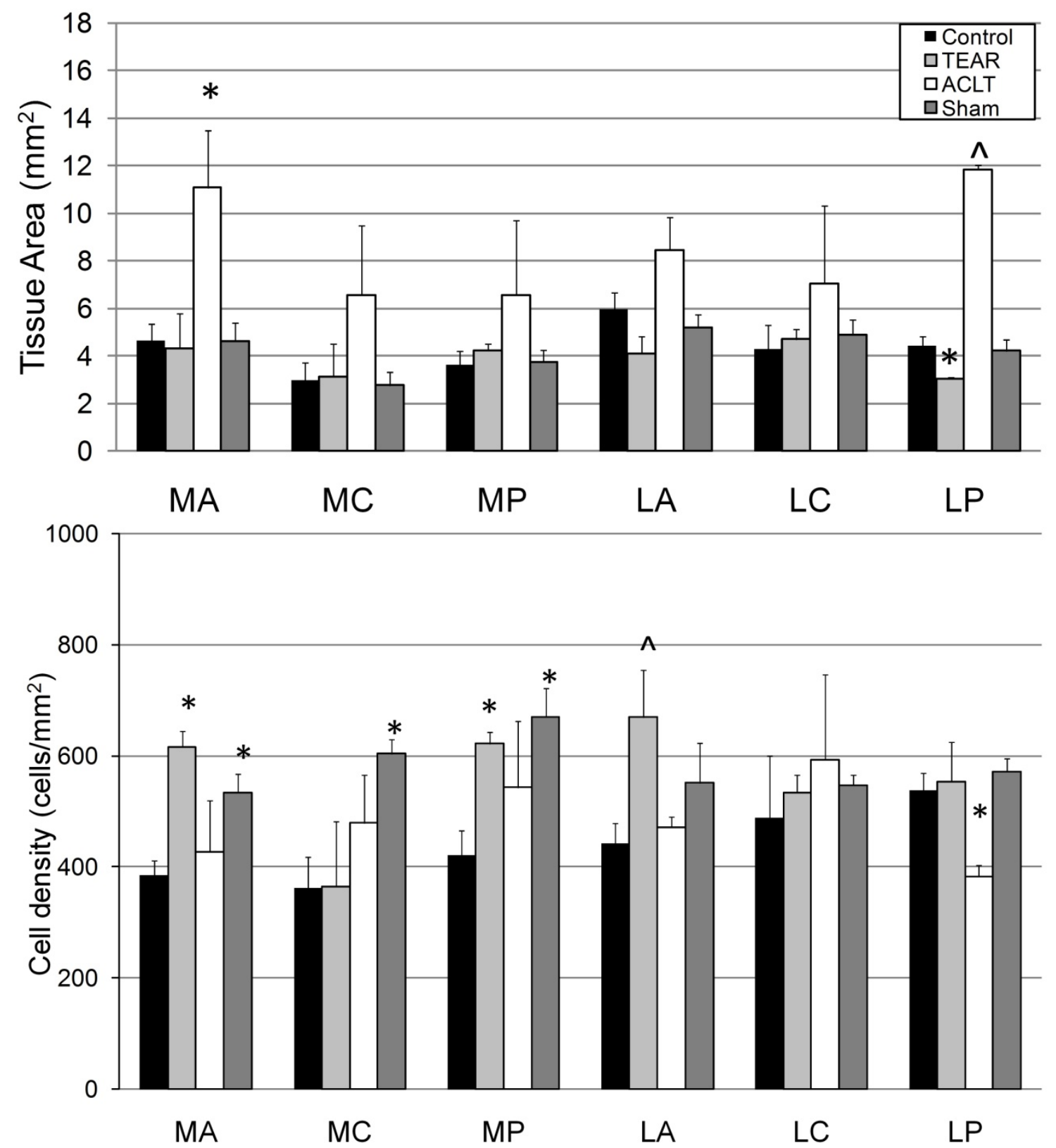

Figure 6-4. Tissue area $\left(\mathrm{mm}^{2}\right)$ and cell density $\left(\right.$ cells $\left./ \mathrm{mm}^{2}\right)$ [mean $\left.+\mathrm{SE}\right]$ for control, TEAR, ACLT, and sham ACLT medial (M) and lateral (L) anterior (A), central (C), and posterior $(\mathrm{P})$ regions. ${ }^{*}=$ significantly lower than control group for same region. ${ }^{\wedge}=$ significantly lower than other two groups for same region. 


\section{Chapter 7 - Acute Cell Viability of the Meniscus and Cartilage and NO Release of Joint Tissues Following Traumatic Impaction}

\section{Abstract}

Objective: Traumatic impaction is known to cause acute cell death and macroscopic damage to cartilage and menisci in vitro. The purpose of this study was to investigate the viability of the medial and lateral menisci and articular cartilage, as well as the release of nitric oxide from these tissues, immediately following traumatic impaction in a closedjoint animal model.

Methods: The left limbs of four rabbits were subjected to tibiofemoral impaction resulting in ACL rupture and both knees were immediately dissected. Cell viability of lateral and medial menisci as well as cartilage was assessed and nitric oxide release to the media was assayed.

Results: A significant decrease in cell viability was observed in the lateral menisci following traumatic impaction compared to control limbs. No significant difference in viability was measured in medial menisci or articular cartilage. No differences in NO release were measured after 12 and $24 \mathrm{hrs}$ of incubation.

Conclusion: This is the first study to investigate acute meniscal viability and NO release following traumatic impaction in vivo. The changes in cell viability of the lateral menisci may suggest its role in advancement of damage in the traumatic ACL ruptured knee.

\section{Introduction}

Traumatic impaction is known to cause acute cell death and macroscopic damage to cartilage and menisci in vitro ${ }^{151,152,282,305}$. It is understood that damage to the menisci can lead to chronic problems associated with excessive cartilage wear and the eventual onset of osteoarthritis $(\mathrm{OA})^{231}$. Additionally, cartilage fissuring, subchondral bone bruising, and chondrocyte death are also believed to lead to the rapid progression of joint degeneration $^{311,339}$. Such injuries, along with posterolateral meniscal tearing, are often 
observed after traumatic impaction and anterior cruciate ligament (ACL) rupture ${ }^{235,322,}$ 338. Therefore, understanding how the menisci and cartilage behaves in the acute response to impaction may help guide future therapies following traumatic knee injury in order to prevent the development of OA.

The purpose of this study was to investigate the viability of the medial and lateral menisci and articular cartilage, as well as investigate the release of nitric oxide (NO) from these tissues, following traumatic impaction in a closed-joint animal model. It was hypothesized that the lateral menisci would demonstrate a significant increase in cell death following traumatic impaction based on its high risk of tearing following traumatic impaction $^{235}$. Additionally, it was hypothesized that the impacted limbs would demonstrate a greater release of NO than the control limbs.

\section{Materials and Methods}

\section{Traumatic Impaction}

Four skeletally mature Flemish Giant rabbits $(5.9 \pm 0.9 \mathrm{~kg})$ were used in the study. The investigation was approved by the Michigan Technological University Institutional Animal Care and Use Committee. All animals were housed in individual cages $\left(61 \times 122 \times 46 \mathrm{~cm}^{3}\right)$ prior to the study. Animals were tranquilized with $1 \mathrm{mg} / \mathrm{kg}$ acepromazine and anesthetized using 5\% isoflorane and 1\% oxygen. Animals were euthanized by either overdose of isoflorane or intracardial injection of potassium chloride. Immediately following death, animals received a blunt force insult to the left tibiofemoral (TF) joint using a previously described drop tower ${ }^{235}$. The drop tower sled was arrested electronically after one impact. A pre-crushed, deformable impact head (Hexcel, 3.76MPa crush strength) was used to ensure uniform loading over the femur. The impact interface was mounted in front of a $4.45 \mathrm{kN}$ (1000lb) load transducer (ICP® force sensor, model 208C04, PCB Electronics, Depew, New York, USA) (Figure 7-1). In the current study, the impact mass was $1.75 \mathrm{~kg}$ and was dropped from $0.88 \mathrm{~m}$. The impact force to induce ACL rupture was $737.5 \pm 11.9 \mathrm{~N}$ (mean $\pm \mathrm{SE}$ ). The animal was laid supine with the knee flexed at $90 \mathrm{deg}$. The foot was fixed in a boot with two Velcro straps. Two 
additional Velcro straps crossed over the femur. The left leg was positioned such that the dropped mass struck the distal femur, which resulted in a "kissing" impaction of the femur onto the posterior tibial plateau (Figure 7-1). This impaction encouraged anterior tibial subluxation and ACL tearing. After impaction, an anterior drawer test was used to diagnose ACL tears. The right leg served as an unimpacted control.

Following impaction, both impacted and control legs were disarticulated and dissected under sterile conditions. Surrounding musculature and ligamentous attachments were removed except the ACL, posterior cruciate ligament (PCL) and menisci. Both ACL and PCL ligaments were inspected for tears, and then fully transected at the proximal ends if necessary. The knee was completely disarticulated to investigate the presence and location of meniscal tears. The posterolateral meniscal attachments were detached from the femur using a scalpel. The tibial plateau and femoral condyles were then harvested approximately $0.6-0.8 \mathrm{~cm}$ from the proximal and distal ends, respectively, using a highspeed motorized rotary tool (Dremel, Racine, WI, USA) while continuously rinsing with $37^{\circ} \mathrm{C}$ sterile PBS to avoid the influence of heat on the viability of articular cartilage/meniscal cells. The menisci remained attached to the tibial plateau. Once removed, the ends of the tibia and femur were rinsed twice in PBS and twice in growth media ( $44 \% \mathrm{Hams} / \mathrm{F} 12,10 \%$ fetal bovine serum, and $2 \%$ penicillin/streptomycin; $37^{\circ} \mathrm{C}$ ). Following rinsing, ends were submerged into individual wells containing $10 \mathrm{~mL}$ growth media and incubated at $37^{\circ} \mathrm{C}$ and $5 \% \mathrm{CO}_{2}$. Media was removed at 12 hours post-harvest $\left(\mathrm{t}_{12}\right)$, stored at $-80^{\circ} \mathrm{C}$, and replaced with fresh media. Media was again removed at 24hours post-harvest and stored at $-80^{\circ} \mathrm{C}\left(\mathrm{t}_{24}\right)$.

\section{Cell viability}

At 24hours post-harvest, the menisci were removed from the tibial plateau and a custom drop slicer was used to remove 2-3 parallel $150 \mu \mathrm{m}$ thick coronal sections from the central regions of the lateral (LM) and medial (MM) menisci. This period of incubation has been shown to maximize cell death after traumatic insult in vitro ${ }^{340}$. If a meniscal tear was present, slices were obtained to include the tear while remaining as centrally located as possible. The cut surfaces of the tibial plateau (TPC) and femoral condyles (FC) were 
adhered to Plexiglass squares using cyanoacrylate and 2-3 $150 \mu \mathrm{m}$ thick coronal sections of the cartilage and bone from regions at or slightly posterior of the PCL insertion (TPC) and of the posterior condyle (FC) were obtained using a high-speed isomet saw (Isomet 1000 Precision saw, Buehler, Lake Bluff, IL), with constant hydration of PBS. Sections of menisci and cartilage (TPC and FC) were immediately incubated in $2 \mathrm{mM}$ ethidium homodimer-1 and 4mM Calcein AM in PBS and incubated for 30-45minutes in the dark at room temperature (. Images were captured for both live (green fluorescence) and dead (red fluorescence) cells using a fluorescence microscope (Olympus AX70 microscope and DP70 camera). CellC was used to count live and dead cells from individual images $^{341}$, where the percentage of cell viability was equal to the amount of live cells divided by the total sum of dead and live cells. Data from the 2-3 slices on each anatomical location were averaged, and the data from each animal were compared for statistical analyses $(\mathrm{n}=4)$.

\section{Nitric oxide release}

The release of $\mathrm{NO}$ into the tibial and femoral culture media at $t_{12}$ and $t_{24}$ was quantified using a commercially available assaying kit (Nitrate/Nitrite Colorimetric Assay Kit, Cayman Chemical Company, Ann Arbor, MI) ${ }^{32}$. Quantification of total NO production included the sum concentration of both nitrite and nitrate. The assay measured the total NO concentration in two steps. First, nitrate was converted to nitrite utilizing nitrate reductase. Second, nitrite was converted to a deep purple azo compound through Griess reaction. Absorbance of colored azo was measured and then converted to total NO concentration $(\mathrm{mM})$ using a standard curve. The final concentration was normalized to wet weight ( $\mathrm{g}$ ) of the tissue ( $\mathrm{n}=3$ for each time point). Since proteins are known to interfere with Griess reaction, conditioned media were filtered using a $10 \mathrm{kD}$ cut-off filter before assay (Millipore Microcon YM-10, Bedford, MA, USA).

\section{Statistics}

Statistical analysis was performed to determine differences between control and impacted limbs in cell viability and NO release. A two-way ANOVA with repeated measures was implemented to determine statistical differences in cell viability between anatomical 
location (LM/MM/cartilage) and limb (control/impacted). Post-hoc pair-wise comparisons were performed for detection of differences in MM, LM, and cartilage cell viability within each anatomical location. Pair-wise analyses were also performed for superficial, middle, and deep layers within and between control and impacted limbs. FC sections were not included in statistical analysis due to the limited number of paired slices from control and impacted limbs $(n=1)$. Pair-wise comparisons were also performed to determine differences in release profiles of NO to the media at $t_{12}$ and $t_{24}$ of tibial and femoral tissue from control and impacted limbs. P values $<0.05$ were used for all analyses.

\section{Results}

The morphological assessment of all animals is listed in Table 7-1. Three of the four rabbits experienced ACL tear, although all four were diagnosed with "clicking" prior to joint dissection. Three of the four rabbits experienced lateral meniscal tears, and two had medial tears (Table 7-1). One rabbit experienced both LM and MM tears along with ACL tear (Figure 7-2B). If a meniscal tear was present, it was typically located in the posterior region (Figure 7-2B). Qualitative assessment of synovial tissue indicated that the parameniscal regions of the impacted tissue following a $24 \mathrm{hr}$ period of incubation were more reactive compared to control tissue (Figure 7-2C \& D).

Table 7-1. Morphological assessment including meniscal and ACL tears of animals.

\begin{tabular}{|l|c|c|c|}
\multicolumn{1}{c}{ LM tear } & MM tear & ACL tear \\
\hline A1 & $\checkmark$ & $\checkmark$ & $\checkmark$ \\
\hline A2 & $\checkmark$ & -- & -- \\
\hline A3 & $\checkmark$ & -- & Partial \\
\hline A4 & -- & $\checkmark$ & Partial \\
\hline
\end{tabular}

From a qualitative standpoint, uninjured controls demonstrated consistent and uniform cell viability across menisci and cartilage sections, with dead cells evenly distributed across the sections (Figure 7-3). In tissue from impacted knees, a significant decrease in cell viability was observed in LM compared to that of controls (Figure 7-4). In menisci 
from impacted limbs, increased cell death was noticeable along the tear edges, where cell death was most prominent (Figure 7-3). Changes in cell viability of the MM were not statistically different between control and impacted limbs (Figure 7-4).

In this study, cell viability of the TPC from control and impacted limbs did not differ (Figure 7-4). Additionally, there were no differences in cell viability between control and impacted limbs with respect to superficial, middle, or deep cartilage layers (Figure 7-5). Although cell viability of cartilage did not differ between impacted and control limbs, qualitative differences in cell viability were observed between zones (Figure 7-6). In particular, the superficial zone appeared to have "hot spots" where more cell death was observed for the impacted limbs compared to the control (Figure 7-6B \& D). Additionally, one animal experienced a deep zone cartilage bruise that demonstrated complete cell death in the bruise region following impaction (Figure 7-6F).

The release of NO from femoral and tibial tissue blocks was not significantly influenced by traumatic impaction at either $\mathrm{t}_{12}$ or $\mathrm{t}_{24}$ time points (Figure 7-7). However, the femoral condyles from impacted limbs tended to show a greater release at $t_{12}$ and $t_{24}$ than control limbs (Figure 7-7).

\section{Discussion}

This is the first study to explore changes in cell viability of menisci following traumatic damage in vivo. In this study, cell death of the menisci was localized near and at the surfaces of tears, particularly in the inner zone. Cell death was also most apparent in the lateral menisci. In cartilage, cell death was localized predominantly at bruise sites and at "hot spots" in the superficial zones of impacted limbs. Several others have explored cell viability of cartilage following impaction injuries ${ }^{151,152,282,305}$ and have shown similar findings of cell death along fissures. However, only marginal work has been done to explore how trauma influences cell viability of the menisci ${ }^{305}$. Nonetheless, the accumulation of cell death at tear sites of menisci in the present study was not unexpected, based on previous cartilage studies ${ }^{151,152}$. In the present study, injury was 
induced by anterior tibial subluxation, which led to longitudinal tears in the posterior aspect of the lateral and medial menisci. Recent work by Kisiday et al impacted meniscal explants in vitro and observed a substantial amount of cell death post-impaction without macroscopic damage to the articulating surface of the menisci ${ }^{305}$. Additionally, Isaac et al measured cell viability of cartilage using a similar in vivo model that constrained the tibia and prevented anterior tibial subluxation ${ }^{282}$. In their study, cell viability of the cartilage significantly decreased in medial and lateral compartments following traumatic impaction, and suggested differences in viability between medial and lateral facets ${ }^{282}$. However, their study did not investigate the role of impaction on the viability of the meniscal cells. In the present study, the lack of dead cells in the articular cartilage was surprising. Although we expected to see a decrease in cell viability in cartilage and menisci, significant decreases were only observed for the lateral menisci. It is likely that, because the present model encourages anterior tibial subluxation, the menisci are more susceptible to damage than the cartilage which they protect.

In the present model, the dissipation of energy following impaction may be focused through tears in the menisci and ACL, leaving the cartilage relatively unscathed. As the ACL ruptures and the tibia sublux anteriorly, the menisci also tear; such tearing of both ACL and menisci may encourage a combined "pinched" shearing of the meniscal surface and compression of the femur on the posterior edge of the tibial plateau. Because the menisci and ACL tear, and deformation of these soft tissues is observed, the strain energy may be stored in these tissues rather than being attenuated through to the underlying cartilage. Conversely, in the constrained model, load is transferred axially through the menisci, and the meniscal integrity is conserved; therefore, the strain energy may be transferred to the underlying cartilage ${ }^{282}$. Development of computer models may elucidate the transfer of energy suggested with these two injury modes. From a qualitative standpoint, traumatic impaction and ACL rupture does lead to some cartilage cell death, although this was not found to be significant in the present study. It may also be that the lack of statistical significance of cartilage cell death in the present study may be a result of acquiring combined medial and lateral slices of the tibial plateau as opposed 
to isolated facets, or that the harvesting of 2-3 articular slices in the present study may have missed the cell death of the articular cartilage. Future studies investigating traumatic impaction and anterior tibial subluxation should isolate articular cartilage from both medial and lateral facets as well as harvest more slices for live/dead staining in order to map cell viability across the entire plateau. The current study may have missed visualization of the impaction site and thus missed cell death of the articular cartilage.

Other in vitro impaction studies have used different constraints to investigate how cells of cartilage and menisci respond to impaction. For example, Lewis et al ${ }^{152}$ mimicked stress magnitudes applied to cartilage explants in vitro to those applied by Torzilli et $\mathrm{al}^{342}$, but noted different levels of chondrocyte viability. This was most likely the result of different experimental constraints, as Torzilli et $\mathrm{a}^{342}$ impacted isolated cartilage explants whereas Lewis et $\mathrm{al}^{152}$ impacted intact cartilage-subchondral bone explants. The viability of menisci, albeit only minimally investigated, has also only been explored in explant form $^{99,138,305}$. Additionally, only one of these studies has explored how impactioninduced trauma influences the viability of meniscal cells ${ }^{305}$. Although studying impaction in vitro has allowed researchers to isolate the tissue of interest from external factors, such as synoviocytes, macrophages, and vasculature, it may also change the mechanical behavior of the tissue ${ }^{151,152,342}$. In a modification to the present in vivo model, it has been shown that impaction without anterior tibial subluxation has led to significant decreases in chondrocyte viability of both lateral and medial tibial plateaus ${ }^{282}$. Additionally, constraining the tibia to maintain ACL integrity has led to incongruous pressure mapping across the tibial plateau, with the largest pressure measurements located at the exposed regions of articular cartilage rather than those areas protected by the menisci ${ }^{282}$. In the present study, we encouraged anterior tibial subluxation by not constraining the tibia during femoral impaction and measured cell viability of both cartilage and menisci. Along with ACL rupture, the lack of anterior tibial constraint also encouraged meniscal tears that were not observed in the constrained model (unpublished data). Thus, the present study and previous work by Isaac et $\mathrm{al}^{282}$ has demonstrated two models with 
differing boundary conditions to investigate in vivo traumatic loading of cartilage and menisci.

Although release of NO into the culture media was not statistically significant, trends that suggest a greater release of NO from femoral tissue is encouraged. We would expect to see an increase in both tibial and femoral NO release at both 12 and 24 hours. It is possible that the NO release from the tibial tissue was marked by residual bone marrow and vasculature the cultured tibial ends. In the present study, we hoped to determine whole-joint changes in NO release following impaction. However, the release of NO from cartilage and menisci may have been much less than the normal activity of NO in bone marrow and vasculature. Future culturing methods should isolate cartilage and menisci (eg. in explant form) in order to determine the NO release of each respective tissue following traumatic impaction.

This study illustrates the localized cell death following impaction-induced ligamentous rupture found with meniscal and cartilage defects. An understanding of the cellular response of meniscal tissue to traumatic impaction may lead to alterations in acute treatments and delay progression of meniscal degradation and development of OA.

\section{Recommendations}

Future work should investigate potential mediators of cell viability during the acute phase of injury and their role in short term and chronic meniscal and cartilage health. For example, cell surfactants such as poloxamer-188 may maintain the integrity of the cell membrane and prevent cell death following traumatic impaction. Monitoring cell necrosis and cell apoptosis in the acute traumatically injured knee may also elucidate mechanisms of cell clearing, cell clustering, and matrix degeneration observed in the injured menisci of post-traumatic arthritic knees. 


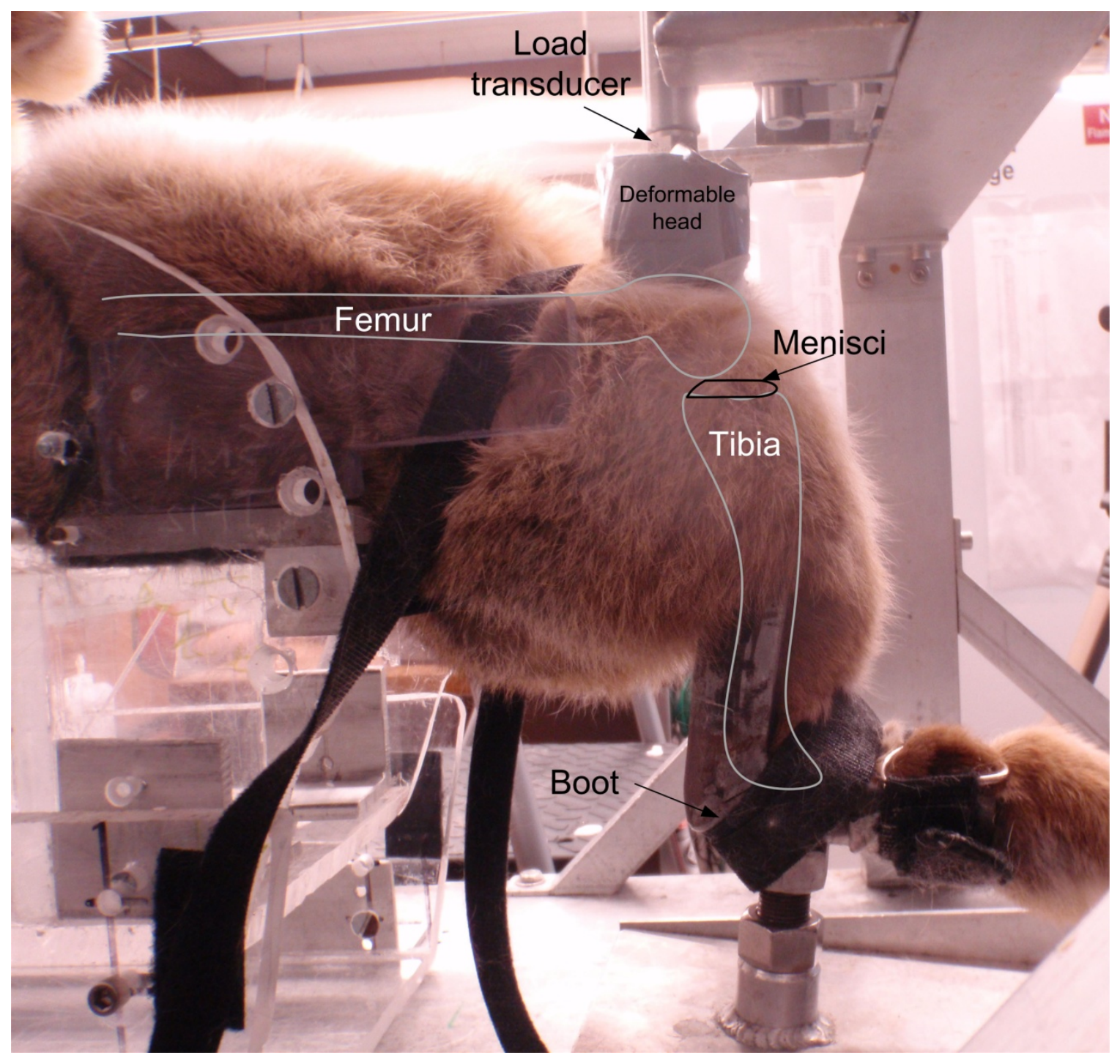

Figure 7-1. Impact experiments were performed by dropping a gravity-accelerated mass onto the flexed tibial-femoral joint. The rabbit knee was positioned such that the deformable head struck the distal femur in order to induce anterior tibial subluxation and ACL rupture. 

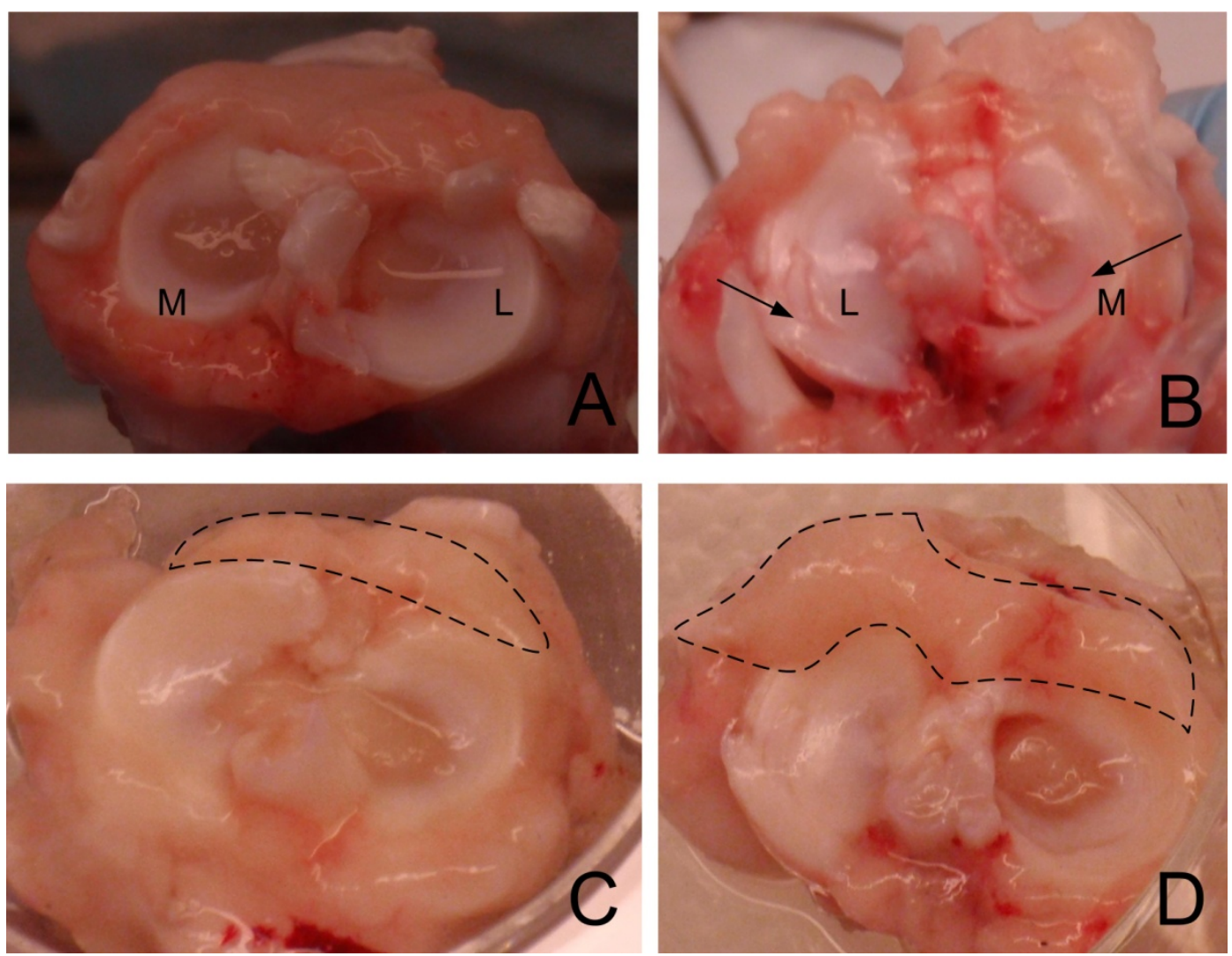

Figure 7-2. Gross morphology of menisci immediately after dissection in control, unimpacted right knee (A) and impacted left knee resulting in ACL tear (B). Reactivity of synovium is marginal after $24 \mathrm{hrs}$ of incubation in tissue harvested from control limbs (C) compared to the swelling of the perimeniscal region of impacted knees at the same postincubation time point (D). Arrows highlight tears in the posterior region of both menisci (B). Synovial reactivity highlighted by area of dashed lines in C \& D. L = lateral, M = medial. 


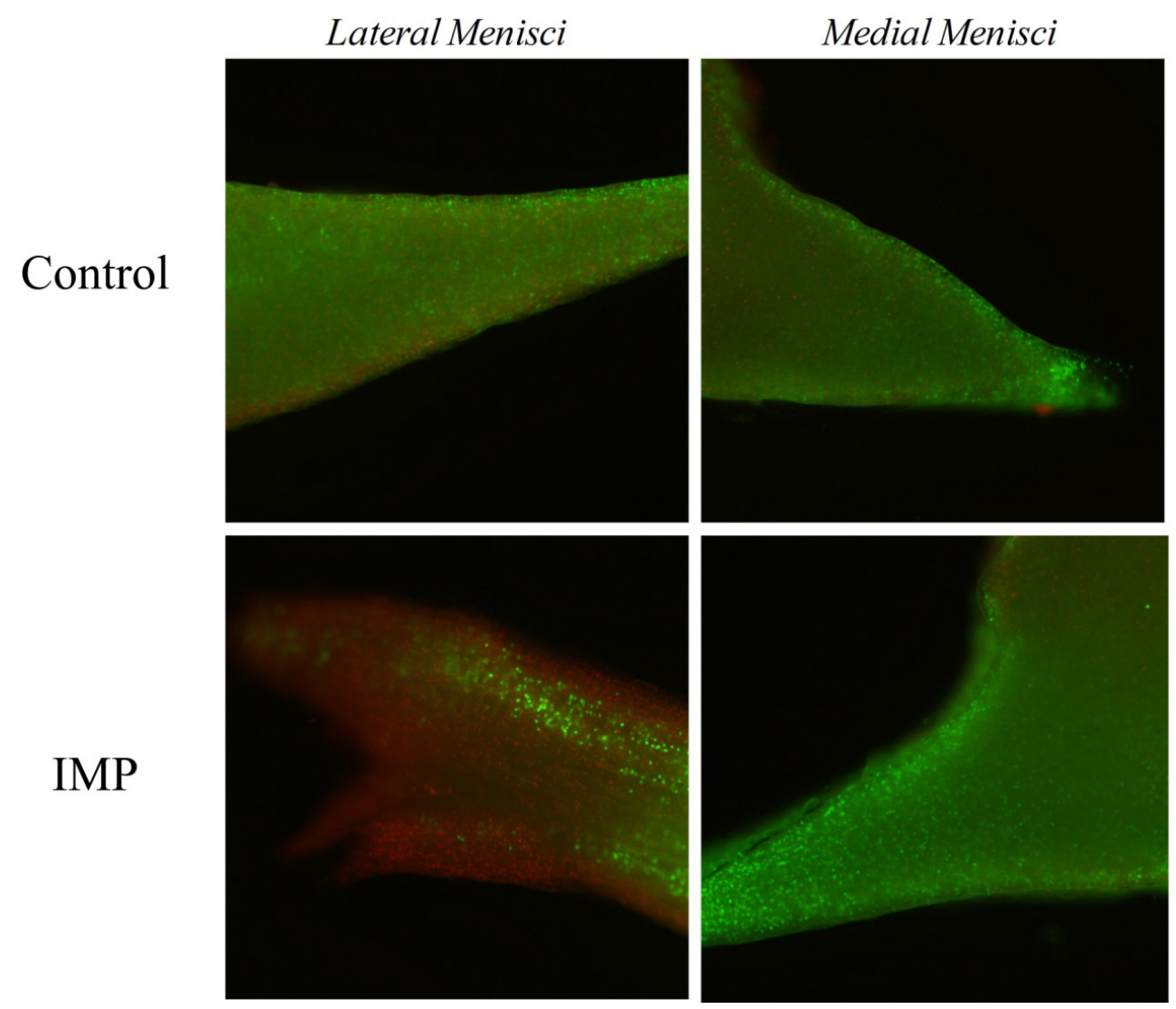

Figure 7-3. Cell viability of lateral and medial menisci from control and impacted knees. Note the increased cell death (red cells) at tear edge of the impacted lateral menisci. 


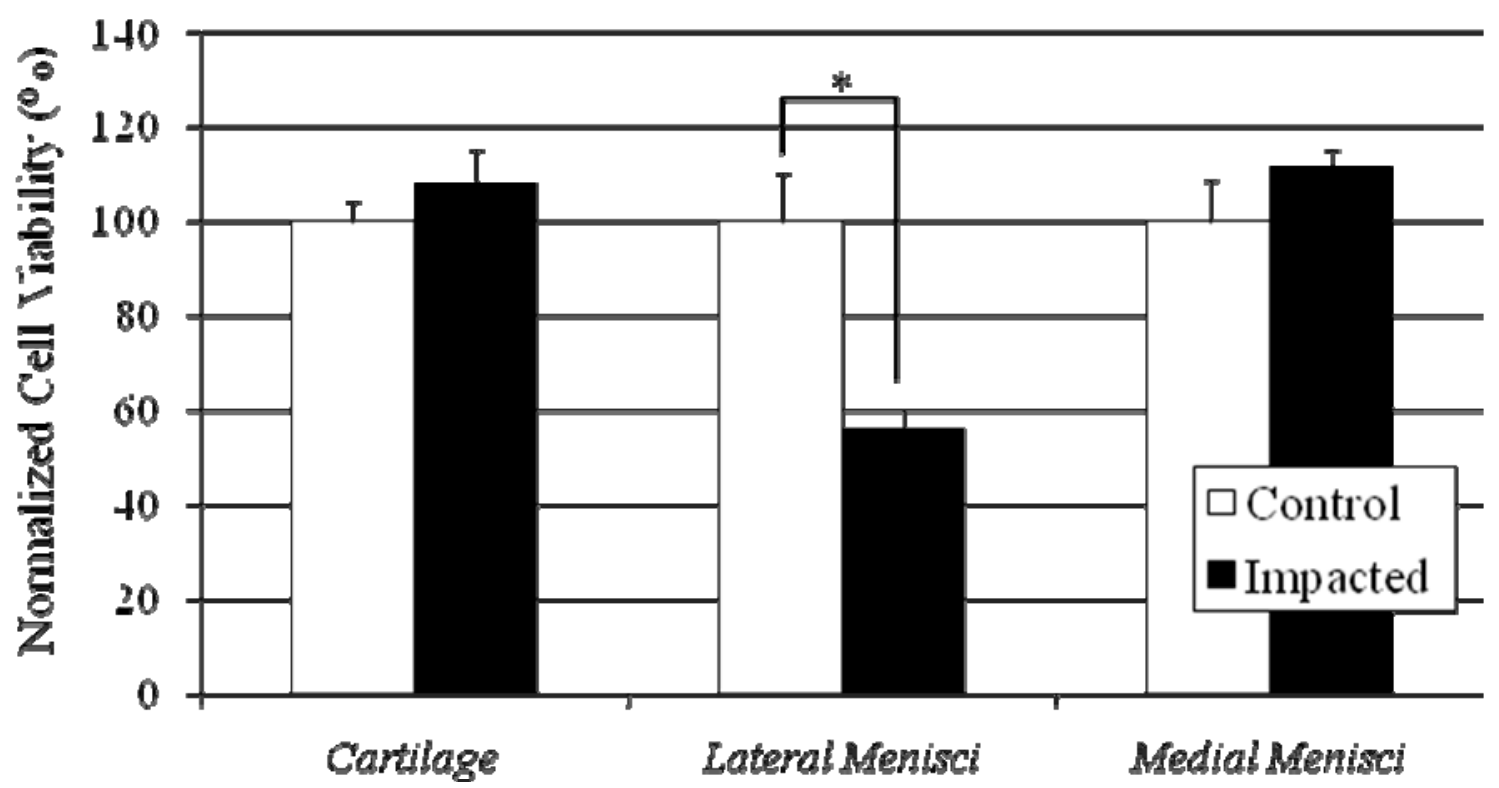

Figure 7-4. Cell viability of cartilage, lateral menisci, and medial menisci normalized to control limb. $*=$ significantly different between groups

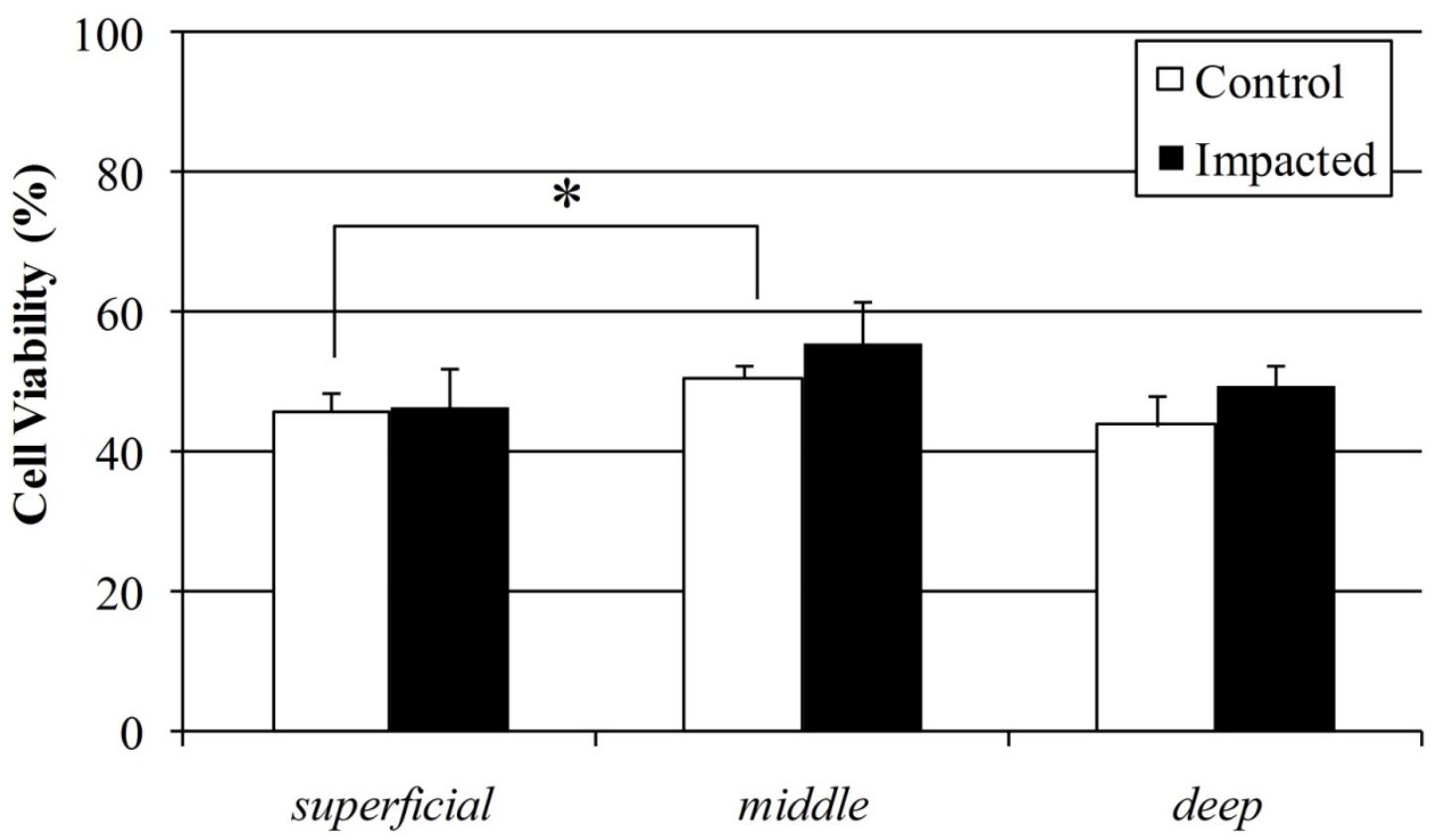

Figure 7-5. Zonal comparison of cell viability of articular cartilage for control and impacted limbs. $*=$ significantly different between zone layers. 


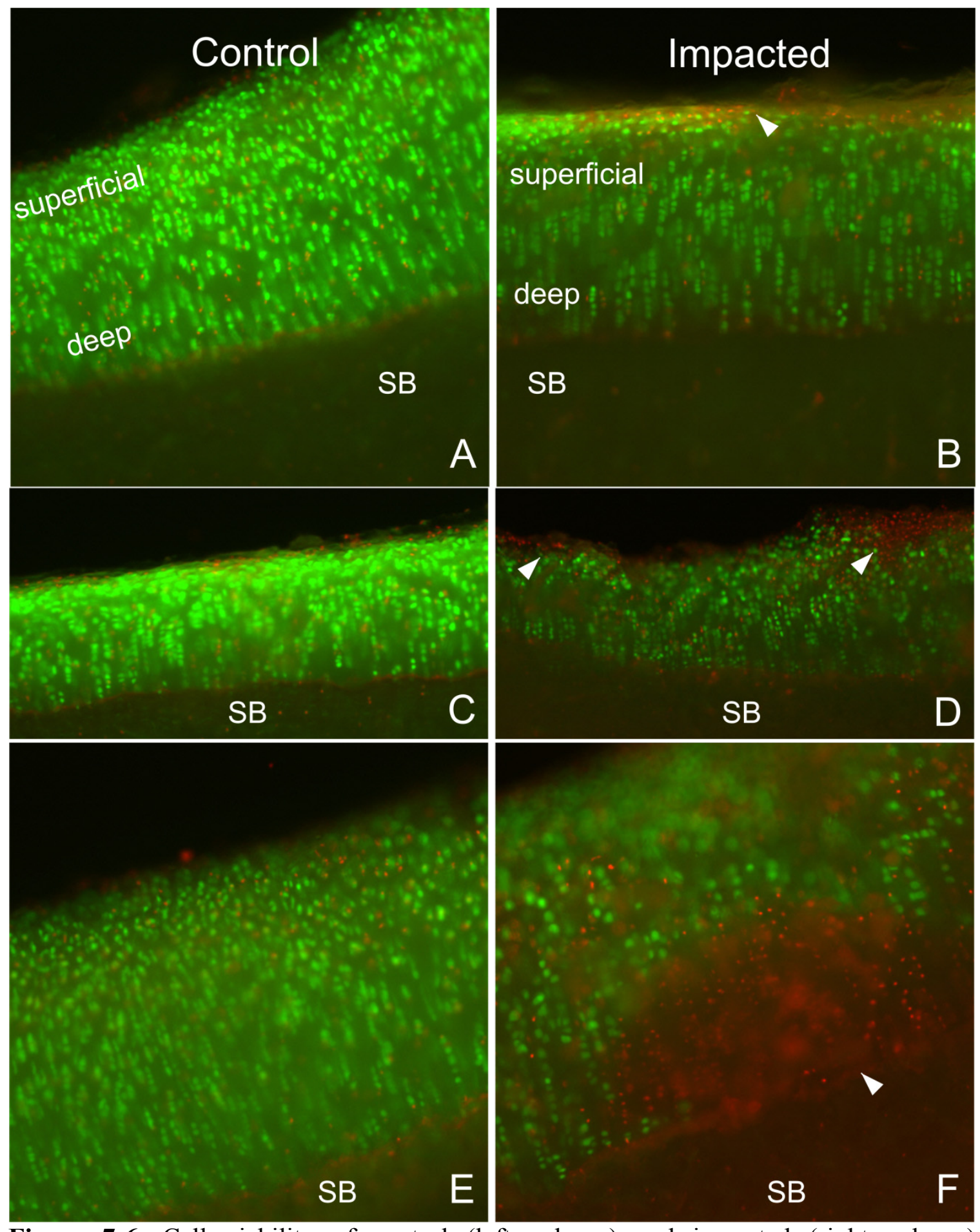

Figure 7-6. Cell viability of control (left column) and impacted (right column) tibiofemoral cartilage. Note the localization of cell death (red cells) in the impacted group, particularly in the superficial (D) and deep bruised (F) regions. Arrows illustrate regions of high cell death. $\mathrm{SB}=$ subchondral bone. 


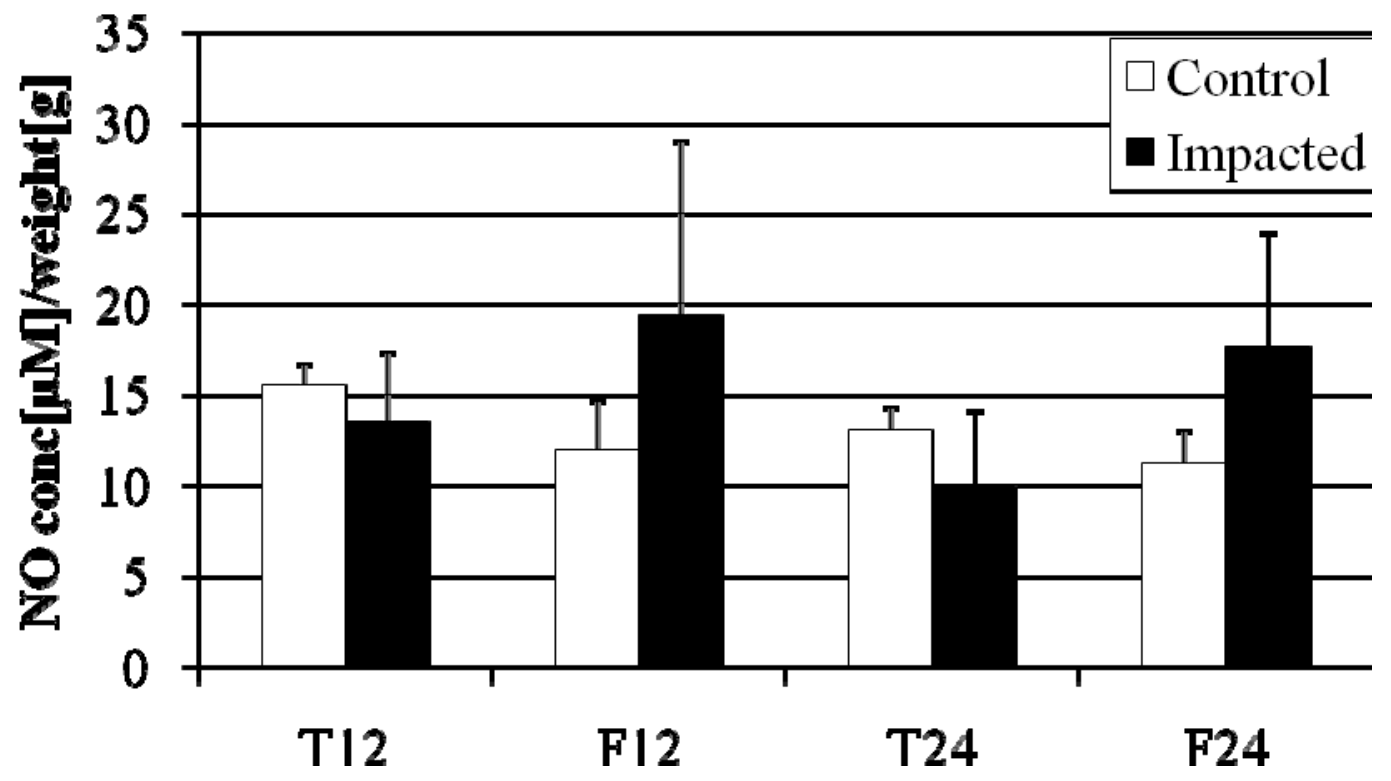

Figure 7-7. NO release of meniscotibial ( $\mathrm{T}$ ) and femoral (F) tissue from control and impacted limbs measured in the culture media after 12 and 24 hours of incubation. NO concentration $(\mu \mathrm{M})$ was normalized to wet weight of tissue $(\mathrm{g})$. 


\section{Chapter 8 - Significance of Research}

\section{Understanding the Etiology of Osteoarthritis}

The influence of osteoarthritis (OA) on the economy, disability, health care, and wellbeing is astounding. Nearly 21 million Americans are affected by this debilitating disease. There are several potential culprits for $\mathrm{OA}^{343}$, including increased age, obesity, joint malalignment, and injury. Of these risk factors, the derivations of degeneration may differ. For example, the knee of an obese patient may develop OA of unknown and/or multifaceted etiology. It may be that chronic overloading of the weight-bearing joints lead to degeneration, or perhaps that joint malalignment accelerates the disease $\mathrm{e}^{344}$. Nonetheless, pinpointing the initiation of OA in many cases is often impossible. However, in knee of an athlete subjected to anterior cruciate ligament (ACL) rupture, damage and altered loading are suspected causes of accelerated OA advancement. Clinically, the progression of OA in the post-traumatic knee is rapid ${ }^{345}$ and imposes on what would otherwise be a healthy knee. However, the mechanisms of degeneration are not clear and must be further investigated.

The viability of cells in the post-traumatized soft tissue has been explored over the last decade $151,152,282,305,342$. However, the work presented in this dissertation is the first of its kind to investigate acute changes to the menisci in the traumatically injured knee. Although acute damage of the menisci in vivo demonstrates increased cell death at tear sites following traumatic impaction, the implications of cell death are not clear. Additionally, the mechanisms of cell death following damage to articular cartilage and menisci are not well understood ${ }^{346,347}$. Although apoptotic death may be induced by traumatic impaction, it is also likely that necrotic cell death is also playing a role in the viability outcomes of torn menisci. Cell necrosis leads to cell swelling, organelle spillage to the extracellular matrix, and the subsequent induction of inflammatory pathways ${ }^{348}$. However, apoptosis is typically more metabolically quiet, and does not lead to any substantial inflammatory response ${ }^{348}$. It is likely that the majority of dead cells observed 
in the torn menisci are necrotic based on their clustering and density. Along similar lines, the effect of cell clearing, macrophage activation, and the ability of injured tissue to heal following injury has only been marginally investigated ${ }^{99,}$ 137, 349-351. Future studies designed to follow the progression of cell death, clearing, and repair in soft tissues of the traumatized joint are needed.

Knee injury in animal models likely lead to an increase in inflammatory mediators and joint effusion ${ }^{13,28,33,35,104,315}$. In clinical studies, inflammatory mediators were found in synovial fluids of patients with $\mathrm{OA}^{352}$, 353. Inflammatory mediators have been demonstrated to encourage the recruitment of cytokines and promote imbalance in anabolic/catabolic transcriptional homeostasis. These molecules have been inhibited in clinical trials by the application of non-steroidal anti-inflammatory drugs (NSAID) ${ }^{353}$. NSAIDS typically only inhibit specific pathways, such as the COX-2 or PGE-2 pathways ${ }^{353}$. Also, adverse effects with NSAID use are sometimes observed, including hypertension, gastrointestinal hemorrhage and anemia, and many clinicians are resorting to COX-2 inhibitors ${ }^{354}$. Unfortunately, selective COX-2 inhibitors may increase the risk of myocardial infarction ${ }^{354}$. These drugs are typically used after OA has symptomatically developed. However, inhibiting inflammation during the acute progression of posttraumatic OA has not yet been thoroughly explored.

It is advantageous to investigate the mechanisms that initiate OA so as to prevent its progression during early-stage development. Severe advancement of OA is typically remedied with total knee arthroplasty, of which components may only last fifteen or so years. Advances in tissue engineering remedies are being explored, and surgical techniques are continuing to improve. Currently, it is known that the progression of OA is dependent in part on the mechanical environment of the knee. Understanding how meniscal cells respond to different mechanical loads may help develop mechanisms for retaining mechanical environments post-injury. This may lead to developments in preventative medicine and treatments, including: use of supplements, such as methylsulfonylmethane (MSM) and glucosamine chondroitin; pharmaceuticals driven to 
preserve the membranes of mechanically compressed cells, such as poloxamer-188; and implementation of specific cyclic exercises, such as continuous passive motion therapy, to encourage fibrochondrocyte health and stimulate healthy cell activity. Additionally, encouraging awareness and early treatment modalities of joint injuries is crucial for the health of the post-traumatic knee.

\section{Development of More Reliable Animal Models for Osteoarthritis}

It is common for researchers to use animal models when investigating diseases and injuries. As such, it seems imperative to accelerate disease in animal models, especially if the disease has a slow progression, in order to observe the effects in a suitable time frame. However, some manipulations to accelerate diseases are not realistic for all types of OA development. For example, as the work in this dissertation elucidates, the commonly used ACLT model of OA likely does not replicate the progression of damage observed in the post traumatic knee. Additionally, parapatellar surgery and arthroscopy may also stimulate an immune response in the knee joint space, which may contribute to decreased healing time or impaired joint health. Some researchers have accounted for this in animal studies by using sham operated control animals in ACL-transection (ACLT) studies, in which they open the joint capsule of all animals but do not always surgically transect the ligament. However, it is possible that the sham operation itself triggers synovial cell activation which may encourage damage to other soft tissues within the knee.

The implementation of an animal model that encourages trauma-initiated, closed-joint $\mathrm{OA}$ is in need of being further developed. Future studies investigating the in vivo characteristics of the healthy and post-traumatic knee could provide useful information in understanding the mechanobiology of the menisci. For example, the presence of meniscal tears may lead to different stress distributions on the articular cartilage and remaining menisci. Additionally, the magnitude of strain in the remaining menisci following traumatic tear may also change compared to the intact menisci. The development of minimally invasive pressure and strain transducers may help to 
illustrate localized mechanical parameters involved with in vivo meniscal tears, and advanced imaging technologies, such as implementation of quantum dot and fluoroscopy, may help visualize changes in meniscal strains during impaction.

In summary, this work provides a springboard for future studies investigating biological as well as mechanical parameters following changes in meniscal integrity. In particular, the use of both in vitro and in vivo models has developed a better understanding of the biochemical and morphological changes associated with the menisci following acute damage and chronic degeneration. Lastly, this research has aided in understanding of traumatically-induced damage to the knee, particularly the menisci, and encourages future studies to develop rehabilitatory mechanisms which may lead to the delay or prevention of post-traumatic knee OA. 


\section{Bibliography}

1. Lohmander L, What can we do about osteoarthritis? Arthritis and Rheumatism, 2000, 2,2:95-100.

2. Lohmander L, Englund P, Dahl L, et al., The long-term consequence of anterior cruciate ligament and meniscus injuries. American Journal of Sports Medicine, 2007, 35,10:1756-1769.

3. Griffin $\mathrm{T}$ and Guilak F, The role of mechanical loading in the onset and progression of osteoarthritis. Exercise and Sport Science Review, 2005, 33,4:195-200.

4. Englund M, Roos EM and Lohmander LS, Impact of type of meniscal tear on radiographic and symptomatic knee osteoarthritis: A sixteen-year followup of meniscectomy with matched controls. Arthritis and Rheumatism, Aug 2003, 48,8:2178-87.

5. Roos EM, Roos HP, Ryd L, et al., Substantial disability 3 months after arthroscopic partial meniscectomy: A prospective study of patient-relevant outcomes. Arthroscopy, 2000, 16,6:619-26.

6. Tuckman DV, Bravman JT, Lee SS, et al., Outcomes of meniscal repair: Minimum of 2-year follow-up. Bulletin of the Hospital for Joint Diseases, 2006, 63,3-4:100-4.

7. Verdonk R, Alternative treatments for meniscal injuries. Journal of Bone and Joint Surgery- British, Sep 1997, 79,5:866-73.

8. Berthiaume MJ, Raynauld JP, Martel-Pelletier J, et al., Meniscal tear and extrusion are strongly associated with progression of symptomatic knee osteoarthritis as assessed by quantitative magnetic resonance imaging. Annals of Rheumatic Diseases, Apr 2005, 64,4:556-63.

9. Kamekura S, Hoshi K, Shimoaka T, et al., Osteoarthritis development in novel experimental mouse models induced by knee joint instability. OsteoArthritis and Cartilage, 2005, 13,7:632-641.

10. Hellio Le Graverand MP, Vignon, E, Otterness, IG, and Hart, D. Early changes in lapine menisci during osteoarthritis development: Part I: Cellular and matrix alterations. OsteoArthritis and Cartilage, 2001, 9,56-64. 
11. Kobayashi K, Mishima H, Hashimoto S, et al., Chondrocyte apoptosis and regional differential expression of nitric oxide in the medial meniscus following partial meniscectomy. Journal of Orthopaedic Research, 2001, 19,802-808.

12. Takahashi K, Hashimoto S, Kubo T, et al., Hyaluronan suppressed nitric oxide production in the meniscus and synovium of rabbit osteoarthritis model. Journal of Orthopaedic Research, May 2001, 19,3:500-3.

13. Bluteau G, Gouttenoire, J, Conrozier, T, Mathieu, P, Vignon, E, Richard, M, Herbage, D, and Mallein-Gerin, F. Differential gene expression analysis in a rabbit model of osteoarthritis induced by anterior cruciate ligament (ACL) section. Biorheology, 2002, 39,247-258.

14. Fife RS, Brandt KD, Braunstein EM, et al., Relationship between arthroscopic evidence of cartilage damage and radiographic evidence of joint space narrowing in early osteoarthritis of the knee. Arthritis and Rheumatism, Apr 1991, 34,4:377-82.

15. Hunter DJ, Zhang YQ, Tu X, et al., Change in joint space width; hyaline articular cartilage loss or alteration in meniscus? Arthritis and Rheumatism, 2006, 54,8:24882495.

16. Jones AO, Houang MT, Low RS, et al., Medial meniscus posterior root attachment injury and degeneration: MRI findings. Australasian Radiology, Aug 2006, 50,4:30613.

17. Baker P, Coggon, D., Reading, I., Barrett, D., Mclaren, M., and Cooper, C. , Sports injury, occupational physical activity, joint laxity, and meniscal damage. The Journal of Rheumatology, 2002, 29,557-563.

18. Dehaven KE, Diagnosis of acute knee injuries with hemarthrosis. American Journal of Sports Medicine, Jan-Feb 1980, 8,1:9-14.

19. Hagood S, Solomonow M, Baratta R, et al., The effect of joint velocity on the contribution of the antagonist musculature to knee stiffness and laxity. American Journal of Sports Medicine, Mar-Apr 1990, 18,2:182-7.

20. Hearon BF, Brinkley JW, Raddin JH, Jr., et al., Knee ligament injury during lateral impact. Aviation Space \& Environmental Medicine, 1985, 56,1:3-8. 
21. Kannus P, Bergfeld J, Jarvinen M, et al., Injuries to the posterior cruciate ligament of the knee. Sports Medicine (Aukland), Aug 1991, 12,2:110-31.

22. Mcbride ID and Reid JG, Biomechanical considerations of the menisci of the knee. Canadian Journal of Sport Science, Dec 1988, 13,4:175-87.

23. Shoemaker SC and Markolf KL, The role of the meniscus in the anterior-posterior stability of the loaded anterior cruciate-deficient knee. Effects of partial versus total excision. Journal of Bone and Joint Surgery: America, Jan 1986, 68,1:71-9.

24. Palmieri-Smith $\mathrm{R}$ and Thomas A, A neuromuscular mechanism of posttraumatic osteoarthritis associated with ACL injury. Exercise and Sport Science Review, 2009, 37,3:147-53.

25. Hewett TE, Myer GD and Ford KR, Anterior cruciate ligament injuries in female athletes: Part 1, mechanisms and risk factors. American Journal of Sports Medicine, Feb 2006, 34,2:299-311.

26. Pap G, Machner A and Awiszus F, Strength and voluntary activation of the quadriceps femoris muscle at different severities of osteoarthritic knee joint damage. Journal of Orthopaedic Research, Jan 2004, 22,1:96-103.

27. Allen CR, Wong EK, Livesay GA, et al., Importance of the medial meniscus in the anterior cruciate ligament-deficient knee. Journal of Orthopaedic Research, 2000, 18,1:109-15.

28. Appleton CTG, Pitelka V, Henry J, et al., Global analyses of gene expression in early experimental osteoarthritis. Arthritis and Rheumatism, 2007, 56,6:1854-1868.

29. Bachrach NM, Valhmu WB, Stazzone E, et al., Changes in proteoglycan synthesis of chondrocytes in articular cartilage are associated with the time-dependent changes in their mechanical environment. Journal of Biomechanics, Dec 1995, 28,12:1561-9.

30. Fukubayashi $\mathrm{T}$ and Kurosawa $\mathrm{H}$, The contact area and pressure distribution pattern of the knee. A study of normal and osteoarthrotic knee joints. Acta Orthopaedica Scandinavica, 1980, 51,6:871-879.

31. Goldring SR and Goldring MB, The role of cytokines in cartilage matrix degeneration in osteoarthritis. Current Orthopaedic Practice, 2004, 427,S27-S36. 
32. Gupta T, Zielinska B, Mchenry J, et al., IL-1 and iNOS gene expression and NO synthesis in the superior region of meniscal explants are dependent on the magnitude of compressive strains. OsteoArthritis and Cartilage, Oct 2008, 16,10:1213-9.

33. Matyas JR, Ehlers PF, Huang D, et al., The early molecular natural history of experimental osteoarthritis. Arthritis and Rheumatism, 1999, 42,5:993-1002.

34. Smith Jr, GN , The role of collagenolytic matrix metalloproteinases in the loss of articular cartilage in osteoarthritis. Frontiers in Bioscience, 2006, 11,3081-3095.

35. Stoker AM, Cook JL, Kuroki K, et al., Site-specific analysis of gene expression in early osteoarthritis using the pond-nuki model in dogs. Journal of Orthopaedic Surgery and Research, 2006, 1,8: online.

36. Sweigart MA and Athanasiou KA, Toward tissue engineering of the knee meniscus. Tissue Engineering, Apr 2001, 7,2:111-29.

37. Wildey GM, Billetz AC, Matyas JR, et al., Absolute concentrations of mrna for type i and type vi collagen in the canine meniscus in normal and acl-deficient knee joints obtained by rnase protection assay. Journal of Orthopaedic Research, 2001, 19,650658.

38. Yao J, Snibbe J, Maloney M, et al., Stresses and strains in the medial meniscus of an acl deficient knee under anterior loading: A finite element analysis with image-based experimental validation. Journal of Biomechanical Engineering, Feb 2006, 128,1:13541.

39. Gardner E and O'rahilly R, The early development of the knee joint in staged human embryos. Journal of Anatomy, Jan 1968, 102,Pt 2:289-99.

40. Fukazawa I, Hatta T, Uchio Y, et al., Development of the meniscus of the knee joint in human fetuses. Congenital Anomalies, 2009, 49,27-32.

41. Salter D, Godolphin J and Gourlay M, Chondrocyte heterogeneity: Immunohistologically defined variation of integrin expression at different sites in human fetal knees. Journal of Histochemistry and Cytochemistry, 1995, 43,4:447457. 
42. Clark CR and Ogden JA, Development of the menisci of the human knee joint. Morphological changes and their potential role in childhood meniscal injury. Journal of Bone and Joint Surgery: America, Apr 1983, 65,4:538-47.

43. Mikic B, Johnson TL, Chhabra AB, et al., Differential effects of embryonic immobilization on the development of fibrocartilaginous skeletal elements. Journal of Rehabilitative Research and Development, Mar-Apr 2000, 37,2:127-33.

44. Day B, Mackenzie WG, Shim SS, et al., The vascular and nerve supply of the human meniscus. Arthroscopy, 1985, 1,1:58-62.

45. Assimakopoulos A, Katonis P, Agapitos M, et al., The innervation of the human meniscus. Clinical Orthopedics and Related Research, 1992, 275,232-236.

46. Guisasola I, Vaquero J and Forriol F, Knee immobilization on meniscal healing after suture: An experimental study in sheep. Clinical Orthopaedics, Feb 2002, 395:227-33.

47. Haapala J, Arokoski JP, Hyttinen MM, et al., Remobilization does not fully restore immobilization induced articular cartilage atrophy. Clinical Orthopaedics, May 1999, 362:218-29.

48. Jurvelin J, Kiviranta I, Tammi M, et al., Softening of canine articular cartilage after immobilization of the knee joint. Clinical Orthopaedics, Jun 1986, 207:246-52.

49. Kiviranta I, Jurvelin J, Tammi M, et al., Weight bearing controls glycosaminoglycan concentration and articular cartilage thickness in the knee joints of young beagle dogs. Arthritis and Rheumatism, Jul 1987, 30,7:801-9.

50. Ochi M, Kanda T, Sumen Y, et al., Changes in the permeability and histologic findings of rabbit menisci after immobilization. Clinical Orthopaedics and Related Research, Jan 1997, 334:305-15.

51. Bray RC, Smith JA, Eng MK, et al., Vascular response of the meniscus to injury: Effects of immobilization. Journal of Orthopaedic Research, 2001, 19,384-390.

52. Brindle T, Nyland J and Johnson DL, The meniscus: Review of basic principles with application to surgery and rehabilitation. Journal of Athletic Training, 2001, 36,2:160-169. 
53. Upton ML, Hennerbichler A, Fermor B, et al., Biaxial strain effects on cells from the inner and outer regions of the meniscus. Connective Tissue Research, 2006, 47,4:20714.

54. Gray JC, Neural and vascular anatomy of the menisci of the human knee. Journal of Orthopaedic and Sports Physical Therapy, Jan 1999, 29,1:23-30.

55. Mcdevitt $\mathrm{CA}$ and Webber RJ, The ultrastructure and biochemistry of meniscal cartilage. Clinical Orthopedics and Related Research, 1990, 252,8-18.

56. Fink C, Fermor B, Weinberg JB, et al., The effect of dynamic mechanical compression on nitric oxide production in the meniscus. OsteoArthritis and Cartilage, 2001, 1-8.

57. Hellio Le Graverand MP, Ou Y, Schield-Yee T, et al., The cells of the rabbit meniscus: Their arrangement, interrelationship, morphological variations and cytoarchitecture. Journal of Anatomy, May 2001, 198,Pt 5:525-35.

58. Verdonk PC, Forsyth RG, Wang J, et al., Characterisation of human knee meniscus cell phenotype. OsteoArthritis and Cartilage, Jul 2005, 13,7:548-60.

59. Wilson CG, Nishimuta JF and Levenston ME, Chondrocytes and meniscal fibrochondrocytes differentially process aggrecan during de novo extracellular matrix assembly. Tissue Engineering Part A., 2009, 15,7:1513-1522.

60. Tumia NS and Johnstone AJ, Promoting the proliferative and synthetic activity of knee meniscal fibrochondrocytes using basic fibroblast growth factor in vitro. American Journal of Sports Medicine, Jun 2004, 32,4:915-20.

61. Webber RJ, Harris MG and Hough AJ, Jr., Cell culture of rabbit meniscal fibrochondrocytes: Proliferative and synthetic response to growth factors and ascorbate. Journal of Orthopaedic Research, 1985, 3,1:36-42.

62. Mauck RL, Martinez-Diaz GJ, Yuan X, et al., Regional multilineage differentiation potential of meniscal fibrochondrocytes: Implications for meniscus repair. Anatomical Record (Hoboken), Jan 2007, 290,1:48-58.

63. Gunja NJ, Uthamanthil RK and Athanasiou KA, Effects of TGF- $\beta 1$ and hydrostatic pressure on meniscus cell seeded scaffolds. Biomaterials, 2009, 30,4:565-573. 
64. Ashwood P, Harvey R, Verjee T, et al., Competition between IL-1, IL -1ra and TGF$\beta 1$ modulates the response of the ela4.Nob-1/ctll bioassay: Implications for clinical investigations. Inflammation Research, 2004, 53,60-65.

65. Segawa Y, Muneta T, Makino H, et al., Mesenchymal stem cells derived from synovium, meniscus, anterior cruciate ligament, and articular chondrocytes share similar gene expression profiles. Journal of Orthopaedic Research, 2009, 27,4:435441.

66. Macconaill M, The functions of intra-articular fibrocartilages with special reference to the knee and inferior radio-ulnar joints. Journal of Anatomy, 1932, 66,210-227.

67. Eifler RL, Dehlin JM, Blough ER, et al., Glycosaminoglycan production in rabbit meniscal cells due to fluid flow. Journal of Orthopaedic Research, 2006, 24,375-384.

68. Djurasovic M, Aldridge JW, Grumbles R, et al., Knee joint immobilization decreases aggrecan gene expression in the meniscus. American Journal of Sports Medicine, May-Jun 1998, 26,3:460-6.

69. Sandy JD, Plaas AHK and Rosenberg L, Koopman WJ, Structure, function, and metabolism of cartilage proteoglycans. In: Arthritis and Allied Conditions: a textbook of Rheumatology. 13th ed. Philadelphia: Williams \& Wilkins; 1997. 223-274.

70. Caterson B, Flannery CR, Hughes CE, et al., Mechanisms involved in cartilage proteoglycan catabolism. Matrix Biology, Aug 2000, 19,4:333-44.

71. Sandy JD, A contentious issue finds some clarity: On the independent and complementary roles of aggrecanase activity and mmp activity in human joint aggrecanolysis. OsteoArthritis and Cartilage, 2006, 14,95-100.

72. Roughley $\mathrm{P}$, The structure and function of cartilage proteoglycans. European Cells and Materials, 2006, 12,92-101.

73. Paulsson M, Morgelin M, Wiedemann H, et al., Extended and globular protein domains in cartilage proteoglycans. Biochemical Journal, 1987, 245,763-772.

74. Melrose J, Fuller ES, Roughley PJ, et al., Fragmentation of decorin, biglycan, lumican and keratocan is elevated in degenerate human meniscus, knee and hip articular cartilages compared with age-matched macroscopically normal and control tissues. Arthritis Research and Therapy, 2008, 10,4:R79. 
75. Blackburn G, Al-Jafary M, Huckerby T, et al., Age-related changes in human meniscal glycosaminoglycans. International Journal of Experimental Biology. 2004, 85:A45-A77.

76. Roughley PJ, Articular cartilage and changes in arthritis: Noncollagenous proteins and proteoglycans in the extracellular matrix of cartilage. Arthritis Research and Therapy, 2001, 3,6:342-347.

77. Mcilwraith W, Anatomy and physiology of equine joints. 2008, 201023 Sept

78. Nakano T, Dodd CM and Scott PG, Glycosaminoglycans and proteoglycans from different zones of the porcine knee meniscus. Journal of Orthopaedic Research, 1997, 15,2:213-220.

79. Anderson DR, Woo SL, Kwan MK, et al., Viscoelastic shear properties of the equine medial meniscus. Journal of Orthopaedic Research, 1991, 9,550-558.

80. Bursac P, Arnoczky S and York A, Dynamic compressive behavior of human meniscus correlates with its extra-cellular matrix composition. Biorheology, 2009, 46,227-237.

81. Adams ME and Ho YA, Localization of glycosaminoglycans in human and canine menisci and their attachments. Connective Tissue Research, 1987, 16,3:269-79.

82. Adams ME, Mcdevitt CA, Ho A, et al., Isolation and characterization of highbuoyant-density proteoglycans from semilunar menisci. Journal of Bone and Joint Surgery; American, 1986, 68,1:55-64.

83. Adams ME and Muir H, The glycosaminoglycans of canine menisci. Biochemical Journal, 1981, 197,2:385-9.

84. Kavanagh E and Ashhurst D, Distribution of biglycan and decorin in collateral and cruciate ligaments and menisci of the rabbit knee joint. Journal of Histochemistry and Cytochemistry, 2001 49,7:877-85.

85. Walker PS and Erkman MJ, The role of the menisci in force transmission across the knee. Clinical Orthopaedics and Related Research, 1975, 109,184-92.

86. Fithian DC, Kelly MA and Mow VC, Material properties and structure-function relationships in the menisci. Clinical Orthopedics and Related Research, 1990, 252,19-31. 
87. Leslie BW, Gardner DL, Mcgeough JA, et al., Anisotropic response of the human knee joint meniscus to unconfined compression. Proceedings of the Instution of Mechanical Engineers [H], 2000, 214,6:631-5.

88. Soltz MA and Ateshian GA, A conewise linear elasticity mixture model for the analysis of tension-compression nonlinearity in articular cartilage. Journal of Biomechanical Engineering, Dec 2000, 122,6:576-86.

89. Mononen ME, Julkunen P, Töyräs J, et al., Alterations in structure and properties of collagen network of osteoarthritic and repaired cartilage modify knee joint stresses. Biomechanics and Modeling in Mechanobiology, 2010, 14 July 2010.

90. Hauch KN, Oyen ML, Odegard GM, et al., Nanoindentation of the insertional zones of human meniscal attachments into underlying bone. Journal of the Mechanical Behavior of Biomedical Materials, 2009, 2,339-347.

91. Rho J-Y and Pharr GM, Effects of drying on the mechanical properties of bovine femur measured by nanoindentation. Journal of Materials Science: Materials in Medicine, 1999, 10,485-488.

92. Oyen ML, Nanoindentation hardness of mineralized tissues. Journal of Biomechanics, 2006, 39,14:2699-702.

93. Rho JY and Pharr GM, Effects of drying on the mechanical properties of bovine femur measured by nanoindentation. Journal of Material Science: Materials in Medicine, Aug 1999, 10,8:485-8.

94. Swadener JG, Rho JY and Pharr GM, Effects of anisotropy on elastic moduli measured by nanoindentation in human tibial cortical bone. Journal of Biomedical Materials Research, Oct 2001, 57,1:108-12.

95. Zielinska B and Haut Donahue TL, 3D finite element model of medial meniscus meniscectomy;changes in contact behavior. Journal of Biomechanical Engineering, 2006, 128,1:115-123.

96. Fabricant P, Rosenberger P, Jokl P, et al., Predictors of short-term recovery differ from those of long-term outcome after arthroscopic partial meniscectomy. Arthroscopy, 2008, 24,7:769-78. 
97. Billante M, Diduch D, Lunardini D, et al., Meniscal repair using an all-inside, rapidly absorbing, tensionable device. Arthroscopy, 2008, 24,7:779-85.

98. Fukuda Y, Takai S, Yoshino N, et al., Impact load transmission of the knee jointinfluence of leg alignment and the role of meniscus and articular cartilage. Clinical Biomechanics (Bristol, Avon), Aug 2000, 15,7:516-21.

99. Mcnulty AL, Moutos FT, Weinberg JB, et al., Enhanced integrative repair of the porcine meniscus in vitro by inhibition of interleukin-1 or tumor necrosis factor alpha. Arthritis and Rheumatism, Sep 2007, 56,9:3033-42.

100. Messner K and Gao J, The meniscus of the knee joint. Anatomical and functional characteristics, and a rationale for clinical treatment. Journal of Anatomy, 1998, 193,161-178.

101. Roos H, Lauren M, Adalberth T, et al., Knee osteoarthritis after meniscectomy: Prevalence of radiographic changes after twenty-one years, compared with matched controls. Arthritis and Rheumatism, 1998, 41,687-93.

102. Choi N, Kim T, Son K, et al., Meniscal repair for radial tears of the midbody of the lateral meniscus. American Journal of Sports Medicine, 2010, Sep 8. [Epub ahead of print].

103. Cetik O, Cirpar M, Eksioglu F, et al., Simultaneous bucket handle tear of both medial and lateral menisci of a knee with chronic anterior cruciate ligament deficiency. Knee Surgery Sports Traumatology and Arthroscopy, 2006, 14,356-359.

104. Hashimoto S, Takahashi K, Ochs RL, et al., Nitric oxide production and apoptosis in cells of the meniscus during experimental osteoarthritis. Arthritis and Rheumatism, Oct 1999, 42,10:2123-31.

105. Burrage PS, Mix KS and Brinckerhoff CE, Matrix metalloproteinases: Role in arthritis. Frontiers in Bioscience, 2006, 11,529-43.

106. Millward-Sadler SJ, Wright MO, Davies LW, et al., Mechanotransduction via integrins and interleukin- 4 results in altered aggrecan and matrix metalloproteinase 3 gene expression in normal, but not osteoarthritic, human articular chondrocytes. Arthritis and Rheumatism, Sep 2000, 43,9:2091-9. 
107. Buschmann MD, Kim YJ, Wong M, et al., Stimulation of aggrecan synthesis in cartilage explants by cyclic loading is localized to regions of high interstitial fluid flow. Archives of Biochemistry and Biophysics, Jun 1 1999, 366,1:1-7.

108. Williams JM, Moran M, Thonar EJ, et al., Continuous passive motion stimulates repair of rabbit knee articular cartilage after matrix proteoglycan loss. Clinical Orthopaedics and Related Research, Jul 1994, 304:252-62.

109. Ferretti M, Srinivasan A, Deschner J, et al., Anti-inflammatory effects of continuous passive motion on meniscal fibrocartilage. Journal of Orthopaedic Research, Sep 2005, 23,5:1165-71.

110. Vailas AC, Zernicke RF, Matsuda J, et al., Adaptation of rat knee meniscus to prolonged exercise. Journal of Applied Physiology, Mar 1986, 60,3:1031-4.

111. Shin SJ, Fermor B, Weinberg JB, et al., Regulation of matrix turnover in meniscal explants: Role of mechanical stress, interleukin-1, and nitric oxide. Journal of Applied Physiology, Jul 2003, 95,1:308-13.

112. Kessler MA, Glaser C, Tittel S, et al., Recovery of the menisci and articular cartilage of runners after cessation of exercise: Additional aspects of in vivo investigation based on 3-dimensional magnetic resonance imaging. American Journal of Sports Medicine, May 2008, 36,5:966-70.

113. Fermor B, Jeffcoat D, Hennerbichler A, et al., The effects of cyclic mechanical strain and tumor necrosis factor alpha on the response of cells of the meniscus. OsteoArthritis and Cartilage, Dec 2004, 12,12:956-62.

114. Upton ML, Chen J, Guilak F, et al., Differential effects of static and dynamic compression on meniscal cell gene expression. Journal of Orthopaedic Research, Nov 2003, 21,6:963-9.

115. Ferretti M, Madhavan S, Deschner J, et al., Dynamic biophysical strain modulates proinflammatory gene induction in meniscal fibrochondrocytes. American Journal of Physiology Cell Physiology, Jun 2006, 290,6:C1610-5.

116. Buschmann MD, Gluzband YA, Grodzinsky AJ, et al., Mechanical compression modulates matrix biosynthesis in chondrocyte/agarose culture. Journal of Cell Science, Apr 1995, 108 ( Pt 4),1497-508. 
117. Hennerbichler A, Fermor B, Hennerbichler D, et al., Regional differences in prostaglandin e2 and nitric oxide production in the knee meniscus in response to dynamic compression. Biochemical and Biophysical Research Communications, 2007, 358,1047-1053.

118. Gupta T and Haut Donahue TL, Role of cell location and morphology in the mechanical environment around meniscal cells. Acta Biomaterialia, 2006, 2,5:483492.

119. Alexopoulos LG, Setton LA and Guilak F, The biomechanical role of the chondrocyte pericellular matrix in articular cartilage. Acta Biomaterialia, 2005, 317325.

120. Guilak F and Mow VC, The mechanical environment of the chondrocyte: A biphasic finite element model of cell-matrix interactions in articular cartilage. Journal of Biomechanics, Dec 2000, 33,12:1663-73.

121. Estrov ZK, Kurzrock R and Talpaz M, Interleukin-1 and its inhibitors: Implications for disease biology and therapy. Cancer Treatment Research, 1995:8051-8082.

122. Pimental E, Handbook of growth factors: Peptide growth factors. CRC Press, 1994, Volume II. Boca Raton, FL.

123. Dinarello C and Thompson R, Blocking IL-1: Interleukin 1 receptor antagonist in vivo and in vitro. Immunology Today 1991, 12,404-410.

124. Smith R, Chin J, Sam L, et al., Biological effects of interleukin-1 receptor antagonist protein on interleukin-1-stimulated cartilage erosion and chondrocyte responsiveness. Arthritis and Rheumatism, 1991, 34,78-83.

125. Arend W, Interleukin-1 receptor antagonist. Discovery, structure and properties. Progressive Growth Factor Research, 1990, 2,193-205.

126. Ollivierre F, Gubler U, Towle CA, et al., Expression of IL-1 genes in human and bovine chondrocytes: A mechanism for autocrine control of cartilage matrix degradation. Biochemical and Biophysical Research Communication, Dec 30 1986, 141,3:904-11.

127. Han RT, Tsui S, and Smith T, Up-regulation of prostaglandin E2 synthesis by interleukin-1beta in human orbital fibroblasts involves coordinate induction of 
prostaglandin-endoperoxide $\mathrm{h}$ synthase- 2 and glutathione-dependent prostaglandin e2 synthase expression. The Journal of Biological Chemistry, 2002, 277,16355-16364.

128. Little $\mathrm{CB}$, Flannery $\mathrm{CR}$, Hughes $\mathrm{CE}$, et al., Aggrecanase versus matrix metalloproteinases in the catabolism of the interglobular domain of aggrecan in vitro. Biochemical Journal, Nov 15 1999, 344 Pt 1,61-8.

129. Pelletier J-P, Martel-Pelletier J and Abramson SB, Osteoarthritis, an inflammatory disease: Potential implication for the selection of new therapeutic targets. Arthritis and Rheumatism, 2001, 44,6:1237-1247.

130. Jacques C, Gosset M, Berenbaum F, et al., The role of IL-1 and IL-1ra in joint inflammation and cartilage degradation. Vitamins and Hormones, 2006, 74,371-403.

131. Williams KE and Olsena DR, Matrix metalloproteinase-1 cleavage site recognition and binding in full-length human type iii collagen. Matrix Biology, July, 28,6:373379.

132. Lee E, Lamplugh L, Kluczyk B, et al., Neoepitopes reveal the features of type ii collagen cleavage and the identity of a collagenase involved in the transformation of the epiphyses anlagen in development. Developmental Dynamics, 2009, 238,6:154763.

133. Miwaa HE, Gerkena TA and Hering TM, Effects of covalently attached chondroitin sulfate on aggrecan cleavage by adamts-4 and mmp-13. Matrix Biology, 2006, 25,8:534-545.

134. Killian M and Haut Donahue TL, Effect of pathological and physiological loads on interleukin-1alpha protein production in porcine menisci. American Society of Mechanical Engineers Summer Bioengineering Conference, 2008, Marco Island, FL.

135. Zielinska B, Killian M, Kadmiel M, et al., Meniscal tissue explants response depends on level of dynamic compressive strain. OsteoArthritis and Cartilage, Jun 2009, 17,6:754-60.

136. Hennerbichler A, Moutos FT, Hennerbichler D, et al., Interleukin-1 and tumor necrosis factor alpha inhibit repair of the porcine meniscus in vitro. OsteoArthritis and Cartilage, Sep 2007, 15,9:1053-60. 
137. Weinberg JB, Fermor B and Guilak F, Nitric oxide synthase and cyclooxygenase interactions in cartilage and meniscus: Relationships to joint physiology, arthritis, and tissue repair. Subcellular Biochemistry, 2007, 42,31-62.

138. Wilusz RE, Weinberg JB, Guilak F, et al., Inhibition of integrative repair of the meniscus following acute exposure to interleukin-1 in vitro. Journal of Orthopaedic Research, Nov 29 2007,

139. Mcnulty AL and Guilak F, Integrative repair of the meniscus: Lessons from in vitro studies. Biorheology, 2008, 45,3-4:487-500.

140. Mcnulty AL, Weinberg JB and Guilak F, Inhibition of matrix metalloproteinases enhances in vitro repair of the meniscus. Clinical Orthopaedics and Related Research, Jun 2009, 467,6:1557-67.

141. Murrell GA, Doland MM, Jang D, et al., Nitric oxide: An important articular free radical. Journal of Bone and Joint Surgery: America, Feb 1996, 78,2:265-74.

142. D'lima DD, Hashimoto S, Chen PC, et al., Human chondrocyte apoptosis in response to mechanical injury. OsteoArthritis and Cartilage, Nov 2001, 9,8:712-9.

143. Kirshenbaum A, Swindle E, Kulka M, et al., Effect of lipopolysaccharide (lps) and peptidoglycan (pgn) on human mast cell numbers, cytokine production, and protease composition. BMC Immunology 2008, 9,45: online.

144. Lee S, Tsou A, Chan H, et al., Glucocorticoids selectively inhibit the transcription of the interleukin 1 beta gene and decrease the stability of interleukin-1 beta mRNA. Proceedings of the National Academy of Sciences of the United States of America, 1988, 85,1204-1208.

145. Habib G, Saliba W and Nashashibi M, Local effects of intra-articular corticosteroids. Clinical Rheumatology, 2010, 29,347-356.

146. Ravaud P, Moulinier L, Giraudeau B, et al., Effects of joint lavage and steroid injection in patients with osteoarthritis of the knee: Results of a multicenter, randomized, controlled trial. Arthritis and Rheumatism, Mar 1999, 42,3:475-82.

147. Murata M, Bonassar LJ, Wright M, et al., A role for the interleukin-1 receptor in the pathway linking static mechanical compression to decreased proteoglycan synthesis 
in surface articular cartilage. Archive of Biochemistry and Biophysics, May 15 2003, 413,2:229-35.

148. Neidhart M, Gay RE and Gay S, Anti-interleukin-1 and anti-cd44 interventions producing significant inhibition of cartilage destruction in an in vitro model of cartilage invasion by rheumatoid arthritis synovial fibroblasts. Arthritis and Rheumatism, 2000, 43,8:1719-1728.

149. Goupille P, Mulleman D and Chevalier X, Is interleukin-1 a good target for therapeutic intervention in intervertebral disc degeneration: Lessons from the osteoarthritic experience. Arthritis Research and Therapy, 2007, 9,110: online.

150. Chevalier X, Goupille P, Beaulieu AD, et al., Intraarticular injection of anakinra in osteoarthritis of the knee: A multicenter, randomized, double-blind, placebocontrolled study. Arthritis and Rheumatism, 2009, 61,3:344-352.

151. Ewers BJ, Dvoracek-Driksna D, Orth MW, et al., The extent of matrix damage and chondrocyte death in mechanically traumatized articular cartilage explants depends on rate of loading. Journal of Orthopaedic Research, Sep 2001, 19,5:779-84.

152. Lewis J, Deloria L, Oyen-Tiesma MT, Jr., Rc, et al., Cell death after cartilage impact occurs around matrix cracks. Journal of Orthopaedic Research, 2003, 21,5:881-887.

153. Batiste DL, Kirkley A, Laverty S, et al., Ex vivo characterization of articular cartilage and bone lesions in a rabbit acl transection model of osteoarthritis using mri and micro-ct. OsteoArthritis and Cartilage, 2004, 12,986-996.

154. Chang DG, Iverson EP, Schinagl RM, et al., Quantitation and localization of cartilage degeneration following the induction of osteoarthritis in the rabbit knee. OsteoArthritis and Cartilage, Sep 1997, 5,5:357-72.

155. Hashimoto S, Takahashi K, Amiel D, et al., Chondrocyte apoptosis and nitric oxide production during experimentally induced osteoarthritis. Arthritis and Rheumatism, Jul 1998, 41,7:1266-74.

156. Hayami T, Pickarski M, Wesolowski GA, et al., The role of subchondral bone remodeling in osteoarthritis: Reduction of cartilage degeneration and prevention of osteophyte formation by alendronate in the rat anterior cruciate ligament transection model. Arthritis and Rheumatism, Apr 2004, 50,4:1193-206. 
157. Robertson CM, Pennock AT, Harwood FL, et al., Characterization of pro-apoptotic and matrix-degradative gene expression following induction of osteoarthritis in mature and aged rabbits. OsteoArthritis and Cartilage, May 2006, 14,5:471-6.

158. Smith GN, Mickler EA, Albrecht ME, et al., Severity of medial meniscus damage in the canine knee after anterior cruciate ligament transection. OsteoArthritis and Cartilage, Apr 2002, 10,4:321-6.

159. Hellio Le Graverand MP, Sciore P, Eggerer J, et al., Formation and phenotype of cell clusters in osteoarthritic meniscus. Arthritis and Rheumatism, Aug 2001, 44,8:1808-18.

160. Hellio Le Graverand MP, Eggerer J, Reno C, et al., Mmp-13 expression in rabbit cartilage and menisci following acl-transection. Transactions of the Orthopaedic Research Society, 2000, 46th, 167.

161. Hellio Le Graverand MP, Vignon, E., Otterness, I. G., and Hart, D, Early changes in lapine menisci during osteoarthritis development. Part ii: Molecular alterations. OsteoArthritis and Cartilage 2001, 9,65-72.

162. Vignon E, Bejui J, Mathieu P, et al., Histological cartilage changes in a rabbit model of osteoarthritis. Journal of Rheumatology, May 1987, 14 Spec No,104-6.

163. Hashimoto S, Creighton-Achermann L, Takahashi K, et al., Development and regulation of osteophyte formation during experimental osteoarthritis. OsteoArthritis and Cartilage, 2002, 10,180-187.

164. Yoshioka M, Coutts RD, Amiel D, et al., Characterization of a model of osteoarthritis in the rabbit knee. OsteoArthritis and Cartilage, Jun 1996, 4,2:87-98.

165. D'lima D, Hermida J, Hashimoto S, et al., Caspase inhibitors reduce severity of cartilage lesions in experimental osteoarthritis. OsteoArthritis and Cartilage, 2006, 54,5:1814-1821.

166. Sandell LJ and Aigner T, Articular cartilage and changes in arthritis. An introduction: Cell biology of osteoarthritis. Arthritis Reseach and Therapy, 2001, 3,2:107-13.

167. Wong $\mathrm{M}$ and Carter DR, Articular cartilage functional histomorphology and mechanobiology: A research perspective. Bone, Jul 2003, 33,1:1-13. 
168. Arroll B and Goodyear-Smith F, Corticosteroid injections for osteoarthritis of the knee: Meta-analysis. British Medical Journal, 2004, 328,23 Mar 2004.

169. Bombardier C, Laine L, Reicin A, et al., Comparison of upper gastrointestinal toxicity of rofecoxib and naproxen in patients with rheumatoid arthritis. Vigor study group. New England Journal of Medicine, Nov 23 2000, 343,21:1520-8.

170. Habib GS, Systemic effects of intra-articular corticosteroids. Clinical Rheumatology, 2009, 28:749-756.

171. Wiggins M, Fadale P, Ehrlich M, et al., Effects of local injection of corticosteroids on the healing of ligaments. A follow-up report. Journal of Bone and Joint Surgery: American, 1995, 77,11:1682-1691.

172. Walsh AJL and Lotz JC, Biological response of the intervertebral disc to dynamic loading. Journal of Biomechanics, 2004, 37,3:329-337.

173. Cao M, Stefanovic-Racic M, Georgescu HI, et al., Generation of nitric oxide by lapine meniscal cells and its effect on matrix metabolism: Stimulation of collagen production by arginine. Journal of Orthopaedic Research, Jan 1998, 16,1:104-11.

174. McHenry JA, Zielinska B and Donahue TL, Proteoglycan breakdown of meniscal explants following dynamic compression using a novel bioreactor. Annals of Biomedical Engineering, Nov 2006, 34,11:1758-66.

175. Hardy MM, Seibert K, Manning PT, et al., Cyclooxygenase 2-dependent prostaglandin e2 modulates cartilage proteoglycan degradation in human osteoarthritis explants. Arthritis and Rheumatism, Jul 2002, 46,7:1789-803.

176. Legrand A, Fermor B, Fink C, et al., Interleukin-1, tumor necrosis factor alpha, and interleukin-17 synergistically up-regulate nitric oxide and prostaglandin E2 production in explants of human osteoarthritic knee menisci. Arthritis and Rheumatism, Sep 2001, 44,9:2078-83.

177. Blanco FJ, Ochs RL, Schwarz H, et al., Chondrocyte apoptosis induced by nitric oxide. American Journal of Pathology, Jan 1995, 146,1:75-85.

178. Clancy RM, Amin AR and Abramson SB, The role of nitric oxide in inflammation and immunity. Arthritis and Rheumatism, Jul 1998, 41,7:1141-51. 
179. Sasaki K, Hattori T, Fujisawa T, et al., Nitric oxide mediates interleukin-1-induced gene expression of matrix metalloproteinases and basic fibroblast growth factor in cultured rabbit articular chondrocytes. Journal of Biochemisty (Tokyo), Mar 1998, 123,3:431-9.

180. Chakraborti S, Mandal M, Das S, et al., Regulation of matrix metalloproteinases: An overview. Molecular and Cell Biochemistry, Nov 2003, 253,1-2:269-85.

181. Imai K, Hiramatsu A, Fukushima D, et al., Degradation of decorin by matrix metalloproteinases: Identification of the cleavage sites, kinetic analyses and transforming growth factor-beta1 release. Biochemistry Journal, Mar 15 1997, 322 ( Pt 3),809-14.

182. Janusz MJ, Little CB, King LE, et al., Detection of aggrecanase- and mmpgenerated catabolic neoepitopes in the rat iodoacetate model of cartilage degeneration. OsteoArthritis and Cartilage, Sep 2004, 12,9:720-8.

183. Majumdar MK, Askew R, Schelling S, et al., Double-knockout of adamts-4 and adamts-5 in mice results in physiologically normal animals and prevents the progression of osteoarthritis. Arthritis and Rheumatism, Nov 2007, 56,11:3670-4.

184. Vincenti MP and Brinckerhoff CE, Transcriptional regulation of collagenase (mmp1, mmp-13) genes in arthritis: Integration of complex signaling pathways for the recruitment of gene-specific transcription factors. Arthritis Research and Therapy, 2002, 4,3:157-64.

185. Goupille P, Jayson MI, Valat JP, et al., Matrix metalloproteinases: The clue to intervertebral disc degeneration? Spine, Jul 15 1998, 23,14:1612-26.

186. Shingleton WD, Ellis AJ, Rowan AD, et al., Retinoic acid combines with interleukin-1 to promote the degradation of collagen from bovine nasal cartilage: Matrix metalloproteinases-1 and -13 are involved in cartilage collagen breakdown. Journal of Cell Biochemistry, Sep 14 2000, 79,4:519-31.

187. Hedbom E and Hauselmann HJ, Molecular aspects of pathogenesis in osteoarthritis: The role of inflammation. Cell and Molecular Life Sciences, Jan 2002, 59,1:45-53. 
188. Bau B, Gebhard PM, Haag J, et al., Relative messenger rna expression profiling of collagenases and aggrecanases in human articular chondrocytes in vivo and in vitro. Arthritis and Rheumatism, Oct 2002, 46,10:2648-57.

189. Tortorella MD, Malfait AM, Deccico C, et al., The role of adam-ts4 (aggrecanase-1) and adam-ts5 (aggrecanase-2) in a model of cartilage degradation. OsteoArthritis and Cartilage, Aug 2001, 9,6:539-52.

190. Yamanishi Y, Boyle DL, Clark M, et al., Expression and regulation of aggrecanase in arthritis: The role of tgf-beta. Journal of Immunology, Feb 1 2002, 168,3:1405-12.

191. Malfait AM, Liu RQ, Ijiri K, et al., Inhibition of adam-ts4 and adam-ts5 prevents aggrecan degradation in osteoarthritic cartilage. Journal of Biological Chemistry, Jun 21 2002, 277,25:22201-8.

192. Martel-Pelletier J, Alaaeddine N and Pelletier JP, Cytokines and their role in the pathophysiology of osteoarthritis. Frontiers in Biosciences, Oct 15 1999, 4,D694-703.

193. Pelletier JP, Dibattista JA, Roughley P, et al., Cytokines and inflammation in cartilage degradation, Rheumatic Diseases Clinics of North America, 1993, 19,3:54568.

194. Donati I, Stredanska S, Silvestrini G, et al., The aggregation of pig articular chondrocyte and synthesis of extracellular matrix by a lactose-modified chitosan. Biomaterials, Mar 2005, 26,9:987-98.

195. Natsu-Ume T, Majima T, Reno C, et al., Menisci of the rabbit knee require mechanical loading to maintain homeostasis: Cyclic hydrostatic compression in vitro prevents derepression of catabolic genes. Journal of Orthopaedic Science, Jul 2005, 10,4:396-405.

196. Fitzgerald JB, Jin M, Dean D, et al., Mechanical compression of cartilage explants induces multiple time-dependent gene expression patterns and involves intracellular calcium and cyclic amp. Journal of Biological Chemistry, May 7 2004, 279,19:19502-11.

197. Lipman NS, Jackson LR, Trudel LJ, et al., Monoclonal versus polyclonal antibodies: Distinguishing characteristics, applications, and information resources. Institute for Laboratory Animal Research, 2005, 46,3:258-268. 
198. Runkel L, Meier W, Pepinsky RB, et al., Structural and functional differences between glycosylated and non-glycosylated forms of human interferon- $\beta$ (ifn- $\beta$ ). Pharmaceutical Research, 1998, 15,4:641-649.

199. Ghadially FN, Lalonde JM and Wedge JH, Ultrastructure of normal and torn menisci of the human knee joint. Journal of Anatomy, Jun 1983, 136 (Pt 4),773-91.

200. Upton ML, Chen J and Setton LA, Region-specific constitutive gene expression in the adult porcine meniscus. Journal of Orthopaedic Research, 24,7:1562-1570.

201. Spindler KP, Mayes CE, Miller RR, et al., Regional mitogenic response of the meniscus to platelet-derived growth factor (pdgf-ab). Journal of Orthopaedic Research, 1995, 13,2:201-7.

202. Vincent TL, Hermansson M, Bolton M, et al., Basic fgf mediates an immediate response of articular cartilage to mechanical injury. Proceedings of the National Academy of Sciences U S A, 2002, 99,12:82459-8264.

203. Tortorella MD, Burn TC, Pratta MA, et al., Purification and cloning of aggrecanase1: A member of the adamts family of proteins. Science, Jun 4 1999, 284,5420:1664-6. 204. Englund M and Lohmander LS, Risk factors for symptomatic knee osteoarthritis fifteen to twenty-two years after meniscectomy. Arthritis and Rheumatism, Sep 2004, 50,9:2811-9.

205. Muzio M, Ni J, Feng P, et al., Irak (pelle) family member irak-2 and myd88 as proximal mediators of IL-1 signaling. Science, 1997, 278,5343:1612-1615.

206. Burrage $\mathrm{P}$ and Brinckerhoff $\mathrm{C}$, Molecular targets in osteoarthritis: Metalloproteinases and their inhibitors. Current Drug Targets, 2007, 8,296-303.

207. Mcintyre KW, Stepan GJ, Kolinsky KD, et al., Inhibition of interleukin 1 (IL-1) binding and bioactivity in vitro and modulation of acute inflammation in vivo by IL-1 receptor antagonist and anti-IL-1 receptor monoclonal antibody. Journal of Experimental Medicine, Apr 1 1991, 173,4:931-9.

208. Schwarznau A, Hanson MS, Sperger JM, et al., IL-1b receptor blockade protects islets against pro-inflammatory cytokine induced necrosis and apoptosis. Journal of Cellular Physiology, 2009, 220,2:341-347. 
209. Pfaffl MW, A new mathematical model for relative quantification in real-time rt-pcr. Nucleic Acids Research, May 1 2001, 29,9:online.

210. Patwari P, Gao G, Lee J, et al., Analysis of adamts4 and mt4-mmp indicates that both are involved in aggrecanolysis in interleukin-1-treated bovine cartilage. OsteoArthritis and Cartilage, 2005, 13,4:269-277.

211. Palmer A, Wilson C, Ej B, et al., Composition-functin relationships during IL-1induced cartilage degradation and recovery. OsteoArthritis and Cartilage, 2009, 17,1029-1039.

212. Wilson C, Palmer A, Zuo F, et al., Selective and non-selective metalloproteinase inhibitors reduce IL-1-induced cartilage degradation and loss of mechanical properties. Matrix Biology, 2007, 26,259-268.

213. Pretzel D, Pohlers D, Weinert S, et al., In vitro model for the analysis of synovial fibroblast-mediated degradation of intact cartilage. Arthritis Research and Therapy, 2009, 11,1:R25.

214. Henrotin YE, De Groote DD, Labasse AH, et al., Effects of exogenous IL-1 beta, TNF-alpha, IL-6, IL-8 and LIF on cytokine production by human articular chondrocytes. OsteoArthritis and Cartilage, Sep 1996, 4,3:163-73.

215. Arend WP, The balance between IL-1 and IL-1ra in disease. Cytokine \& Growth Factor Reviews, 2002, 13,323-340.

216. Dinarello CA, Interleukin-1 and interleukin-1 receptor agonist. Nutrition, 1995, 11,492-4.

217. Clark BD, Bedrosian I, Schindler R, et al., Detection of interleukin 1 alpha and 1 beta in rabbit tissues during endotoxemia using sensitive radioimmunoassays. Journal of Applied Physiology, 1991, 71,2412-2418.

218. Dinarello CA, Biologic basis for interleukin-1 in disease. Blood, Mar 15 1996, 87,6:2095-147.

219. Kydd AS, Reno CR, Tsao HW, et al., Early inflammatory arthritis in the rabbit: The influence of intraarticular and systemic corticosteroids on mrna. The Journal of Rheumatology, 2007, 34,1:130-139. 
220. Abramson SB, Attur M, Amin AR, et al., Nitric oxide and inflammatory mediators in the perpetuation of osteoarthritis. Current Rheumatology Reports, Dec 2001, 3,6:535-41.

221. Chowdhury TT, Bader DL and Lee DA, Dynamic compression counteracts IL-1 beta-induced release of nitric oxide and pge2 by superficial zone chondrocytes cultured in agarose constructs. OsteoArthritis and Cartilage, Sep 2003, 11,9:688-96.

222. Kang Y-L, Mbonye U, Delong C, et al., Regulation of intracellular cyclooxygenase levels by gene transcription and protein degradation. Progress in Lipid Research, 2007, 46,108-25.

223. Huang C-Y, Hung L-F, Liang C-C, et al., COX-2 and inos are critical in advanced glycation end product-activated chondrocytes in vitro. European Journal of Clinical Investigation, 2009, 39,5:417-428.

224. Attur M, Wang H-Y, Byers Kraus V, et al., Radiographic severity of knee osteoarthritis is conditional on interleukin 1 receptor antagonist gene variations. Annals of Rheumatic Diseases, 2010, 69,856-861.

225. Arnoczky S, Dodds J and Wickiewicz T, 1996, Basic science of the knee. IN: McGinty JB, et al (eds), Operative Arthroscopy. $2^{\text {nd }}$ edition, Philadelphia: LippincottRaven, 211-239.

226. Mow VCA, S. P.; Jackson, D. W., IN: Mow VC et al (eds), Knee meniscus basic and clinical foundations. 1992, New York: Raven Press, 17-18.

227. Akizuki SM, Pita JC and Howell DS, Tensile properties of human knee joint cartilage: II. Correlations between weight bearing and tissue pathology and the kinetics of swelling. Journal of Orthopedic Research, 1987, 5,2:173-186.

228. Eisenberg SR and Grodzinsky AJ, Swelling of articular cartilage and other connective tissues: Electromechanochemical forces. Journal of Orthopaedic Research, 1985, 3,2:148-59.

229. Lai WM, Hou JS and Mow VC, A triphasic theory for the swelling and deformation behaviors of articular cartilage. Journal of Biomechanical Engineering, Aug 1991, $113,3: 245-58$. 
230. Myers ER, Lai WM and Mow VC, A continuum theory and an experiment for the ion-induced swelling behavior of articular cartilage. Journal of Biomechanical Engineering, May 1984, 106,2:151-8.

231. Aagaard H and Verdonk R, Function of the normal meniscus and consequences of meniscal resection. Scandavian Journal of Medicine and Science of Sports, Jun 1999, 9,3:134-40.

232. Allen PR, Denham RA and Swan AV, Late degenerative changes after meniscectomy. Factors effecting the knee after operation. Journal of Bone and Joint Surgery [Br], 1984, 66,666-671.

233. Berjon JJ, Munuera L and Calvo M, Degenerative lesions in the articular cartilage after meniscectomy: Preliminary experimental study in dogs. Journal of Trauma, Mar 1991, 31,3:342-50.

234. Ehrlich M, Mankin H, Jones H, et al., Biochemical confirmation of an experimental osteoarthritis model. Journal of Bone and Joint Surgery America, 1975, 57,3:392-396.

235. Killian ML, Isaac DI, Haut RC, et al., Traumatic anterior cruciate ligament tear and its implications on meniscal degradation: A preliminary novel lapine osteoarthritis model. Journal of Surgical Research, 2010, 164,2:234-241.

236. Sweigart MA, Zhu CF, Burt DM, et al., Intraspecies and interspecies comparison of the compressive properties of the medial meniscus. Annals of Biomedical Engineering, 2004, 32,11:1569-1579.

237. Nakano T, Thompson JR and Aherne FX, Distribution of glycosaminoglycans and the nonreducible collagen crosslink, pyridinoline in porcine menisci, Canadian Journal of Veterinary Research, Oct 1986, 50,4:532-6.

238. Chia HN and Hull ML, Compressive moduli of the human medial meniscus in the axial and radial directions at equilibrium and at a physiological strain rate. Journal of Orthopaedic Research, Jul 2008, 26,7:951-6.

239. Jerosch J, And Prymka, M., Proprioceptive deficits of the knee joint after rupture of the medial meniscus. Unfallchirurg, 1997, 100,444-448.

240. Jerosch J, Prymka M and Castro WH, Proprioception of knee joints with a lesion of the medial meniscus. Acta Orthopaedica Belgica, Mar 1996, 62,1:41-5. 
241. Levy IM, Torzilli PA and Warren RF, The effect of medial meniscectomy on anterior-posterior motion of the knee. Journal of Bone Joint Surgery America, Jul 1982, 64,6:883-8.

242. Proctor CS, Schmidt, M.B., Whipple, R.R., Kelly, M.A., Mow, V.C., Material properties of the normal medial bovine meniscus. Journal of Orthopaedic Research, 1989, 7,6:771-782.

243. Bellabarba C, Bush-Joseph, C., and Bach, B., Jr Patterns of meniscal injury in the anterior cruciate-deficient knee; a review of the literature. American Journal of Orthopaedics, 1997, 26,18-23.

244. Cerabona F, Sherman MF, Bonamo JR, et al., Patterns of meniscal injury with acute anterior cruciate ligament tears. American Journal of Sports Medicine, Nov-Dec 1988, 16,6:603-9.

245. Cipolla M, Scala, A., Gianni, E., and Puddu, G. , Different patterns of meniscal tears in acute anterior cruciate ligament (acl) ruptures and in chronic acl-deficient knees. Classification, staging and timing of treatment. Knee Surgery, Sports Traumatology, and Arthroscopy, 1995, 3,130-134.

246. Desmet A, And Graf, B. K. , Meniscal tears missed on mr imaging: Relationship to meniscal tear patterns and anterior cruciate ligament tears. American Journal of Roentgeology 1994, 162,905-911.

247. Duncan JB, Hunter R, Purnell M, et al., Meniscal injuries associated with acute anterior cruciate ligament tears in alpine skiers. American Journal of Sports Medicine, Mar-Apr 1995, 23,2:170-2.

248. Spindler K, Schils, J., Bergfeld, J., Andrish, J., Weiker, G., Anderson, T., Piraino, D., Richmond, B., and Medendorp, S. Prospective study of osseous, articular, and meniscal legions in recent anterior cruciate ligament tears by magentic resonance imaging and arthroscopy. The American Journal of Sports Medicine, 1993, 21,551557.

249. Villegas DF, Maes JA, Magee SD, et al., Failure properties and strain distribution analysis of meniscal attachments. Journal of Biomechanics, 2007, 40,12:2655-62. 
250. Mow VC, Ratcliffe A, Chern KY, et al., Mow V, Arnoczky S and Jackson D, Structure and function relationships of the menisci of the knee. 1992, IN: Mow VC et al (eds), Knee meniscus basic and clinical foundations. New York: Raven Press, 3757.

251. Adams ME and Hukins DW, Mow VC, Arnoczky, S.P., Jackson, D.W. The extracellular matrix of the meniscus. 1992, IN: Mow VC et al (eds), Knee meniscus basic and clinical foundations. New York: Raven Press, 15-28.

252. Cheung H, Distribution of type I, II, III, and V in the pepsin solubilized collagens in bovine menisci. Connective Tissue Research, 1987, 16,4:343-356.

253. Chevrier A, Nelea M, Hurtig MB, et al., Meniscus structure in human, sheep, and rabbit for animal models of meniscus repair. Journal of Orthopaedic Research, 27,9:1197-1203.

254. Eyre DR and Muir H, The distribution of different molecular species of collagen in fibrous, elastic and hyaline cartilages of the pig. Biochemical Journal, Dec 1975, 151,3:595-602.

255. Melrose J, Smith S, Cake M, et al., Comparative spatial and temporal localisation of perlecan, aggrecan and type i, ii and iv collage in the ovine meniscus: An ageing study. Histochemistry and Cell Biology, 2005, 124,225-235.

256. Hennerbichler A, Moutos FT, Hennerbichler D, et al., Repair response of the inner and outer regions of the porcine meniscus in vitro. American Journal of Sports Medicine, May 2007, 35,5:754-62.

257. Dickinson SC, Sims TJ, Pittarello L, et al., Quantitative outcome measures of cartilage repair in patients treated by tissue engineering. Tissue Engineering, 2005, 11,1/2:277-287.

258. Martin I, Obradovic B, Freed L, et al., Method for quantitative analysis of glycosaminoglycan distribution in cultured natural and engineered cartilage. Annals of Biomedical Engineering, 1999, 27,656-662.

259. Messner K and Gao J, The menisci of the knee joint. Anatomical and functional characteristics, and a rationale for clinical treatment. Journal of Anatomy, Aug 1998, 193 (Pt 2), 161-78. 
260. Eyre DR and $\mathrm{Wu} J J$, Collagen of fibrocartilage: A distinctive molecular phenotype in bovine meniscus. FEBS Letters, Jul 25 1983, 158,2:265-70.

261. Quinn T, Dierickx P and Grodzinsky AJ, Glycosaminoglycan network geometry may contribute to anisotropic hydraulic permeability in cartilage under compression. Journal of Biomechanics, 2001, 34,11:1483-1490.

262. Lechner K, Hull ML and Howell SM, Is the circumferential tensile modulus within a human medial meniscus affected by the test sample location and cross-sectional area? Journal of Orthopaedic Research, 2000, 18,6:945-51.

263. Gushue DL, Houck J and Lerner AL, Rabbit knee joint biomechanics: Motion analysis and modeling of forces during hopping. Journal of Orthopaedic Research, Jul 2005, 23,4:735-42.

264. Baratz ME, Fu FH and Mengato R, Meniscal tears: The effect of meniscectomy and of repair on intraarticular contact areas and stress in the human knee. A preliminary report. American Journal of Sports Medicine, 1986, 14,4:270-275.

265. Rangger C, Klestil T, Gloetzer W, et al., Osteoarthritis after arthroscopic partial meniscectomy. American Journal of Sports Medicine, 1995, 23,2:240 - 244.

266. Thelin N, Holmberg S and Thelin A, Knee injuries account for the sports-related increased risk of knee osteoarthritis. Scandanavian Journal of Medicine and Science in Sports, Oct 2006, 16,5:329-33.

267. Vanderploeg E and Grodzinsky A, Can the meniscus affect the nature of a chondrocyte? OsteoArthritis and Cartilage, 2009, 17,8:969-70.2009 May 19.

268. Yao J, Funkenbusch PD, Snibbe J, et al., Sensitivities of medial meniscal motion and deformation to material properties of articular cartilage, meniscus and meniscal attachments using design of experiments methods. Journal of Biomechanical Engineering, Jun 2006, 128,3:399-408.

269. Yao J, Lancianese SL, Hovinga KR, et al., Magnetic resonance image analysis of meniscal translation and tibio-menisco-femoral contact in deep knee flexion. Journal of Orthopaedic Research, May 2008, 26,5:673-84.

270. Fremerey R, Lobenhoffer P, Zeichen J, Skutek M, Bosch U, and Tscherne H, Proprioception after rehabilitation and reconstruction in knees with deficiency of the 
anterior cruciate ligament. The Journal of Bone and Joint Surgery, 2000, 82,B:801806.

271. Jerosch J, And Prymka, M. Proprioception and joint stability. Knee Surgery, Sports Traumatology, and Arthroscopy, 1996, 4,171-179.

272. Renström P, Clinical practice of sports injury prevention and care. 1994, IN: Olympic encyclopaedia of sports medicine, Volume 5, Oxford, Boston: Blackwell Scientific Publications, 727.

273. Muellner T, Nikolic A, and Vecsei V, Recommendations for the diagnosis of traumatic meniscal injuries in athletes. Sports Medicine, 1999, 27,337-345.

274. Slocum DB, And Larson, RL, Rotatory instability of the knee: Its pathogenesis and a clinical test to demonstrate its presence. The Journal of Bone and Joint Surgery, 1968, 50,A:211-225.

275. Muellner T, Weinstabl R, Schabus R, et al., The diagnosis of meniscal tears in athletes: A comparison of clinical and magnetic resonance imaging investigations. The American Journal of Sports Medicine, 1997, 25,1:7-12.

276. Tandogan RN, Taser O, Kayaalp A, Taskiran E, Pinar H, Alparsian B, and Alturfan A. Analysis of meniscal and chondral lesions accompanying anterior cruciate ligament tears: Relationship with age, time from injury, and level of sport. Knee Surgery, Sports Traumatology, and Arthroscopy, 2004, 12,262-270.

277. Papastergiou SG, Koukoulias NE, Mikalef P, Ziogas E, and Voulgaropoulos H, Meniscal tears in the acl-deficient knee: Correlation between meniscal tears and the timing of acl reconstruction. Knee Surgery, Sports Traumatology, and Arthroscopy, 2007, 15,1438-1444.

278. Hede A, Jensen DB, Blyme P, et al., Epidemiology of meniscal lesions in the knee. 1,215 open operations in copenhagen 1982-84. Acta Orthopaedica Scandinavica, 1990, 61,5:435-437.

279. Metcalf M and Barrett G, Prospective evaluation of 1485 meniscal tear patterns in patients with stable knees. American Journal of Sports Medicine, 2004, 32,675-680. 
280. Smith J, And Barrett G, Medial and lateral meniscal tear patterns in anterior cruciate ligament-deficient knees: A prospective analysis of 575 tears. American Journal of Sports Medicine, 2001, 29,415-419.

281. Terzidis IP, Christodoulou A, Ploumis A, Givissis P, Natsis K, and Koimtzis M, Meniscal tear characteristics in young athletes with a stable knee: Arthroscopic evaluation. American Journal of Sports Medicine, 2006, 34,1170-1175.

282. Isaac DI, Meyer EG, and Haut RC, Chondrocyte damage and contact pressures following impact on the rabbit tibiofemoral joint. Journal of Biomechanical Engineering, 2008, 130,4:041018.

283. Matyas JR, Atley L, Ionescu M, Eyre DR, and Poole AR, Analysis of cartilage biomarkers in the early phases of canine experimental osteoarthritis. ,Arthritis Research and Therapy, 2004, 50,543-552.

284. Metcalf R, Arthroscopic meniscal surgery. 1991, IN: Operative Arthroscopy, McGinty JB (ed), Raven: New York, 203-236.

285. Ihara H, Miwa M, Takayanagi K, and Nakayama A. Acute torn meniscus combined with acute cruciate ligament injury. Second look arthroscopy after 3-month conservative treatment. Clinical Orthopaedics and Related Research, 1994, 307,146154.

286. Paletta GJ, Levine D, O'Brien S, Wickiewicz T, and Warren RF, Patterns of meniscal injury associated with acute anterior cruciate ligament injury in skiers. The American Journal of Sports Medicine, 1992, 20,542-547.

287. Shelbourne KD, and Nitz PA, The o'donoghue triad revisited: Combined knee injuries involving anterior cruciate and medial collateral ligament tears. American Journal of Sports Medicine, 1991, 19,474-477.

288. Nikolic DK, Lateral meniscal tears and their evolution in acute injuries of the anterior cruciate ligament of the knee: Arthroscopic analysis. Knee Surgery, Sports Traumatology, and Arthroscopy, 1998, 6,26-30.

289. Atkinson PJ, Cooper TG, Anseth S, et al., Association of knee bone bruise frequency with time postinjury and type of soft tissue injury. Orthopaedics, 2008, 31,440 . 
290. Kaplan PA, Walker CW, Kilcoyne RF, et al., Occult fracture patterns of the knee associated with anterior cruciate ligament tears: Assessment with $\mathrm{mr}$ imaging. Radiology, Jun 1992, 183,3:835-8.

291. Yamato M, Yamagishi T, and Kobayashi T, Mr imaging of bone bruise associated with acl tear. Nippon Igaku Hoshasen Gakkai Zasshi, 1993, 53,23-27.

292. Jean Y-H, Wen Z-H, Chang Y-C, et al., Hyaluronic acid attenuates osteoarthritis development in the anterior cruciate ligament-transected knee: Association with excitatory amino acid release in the joint dialysate. Journal of Orthopaedic Research, 2006, 24,5:1052-1061.

293. Mansour JM, Wentorf FA, and Degoede KM, In vivo kinematics of the rabbit knee in unstable models of osteoarthritis. Annals of Biomedical Engineering, 1998, 26,353-360.

294. Reuben SS, And Sklar J, Pain management in patients who undergo outpatient arthroscopic surgery of the knee. Journal of Bone and Joint Surgery, 2000, 82,17541766.

295. Naranje S, Mittal R, Nag H, and Sharma R. Arthroscopic and magnetic resonance imaging evaluation of meniscus lesions in the chronic anterior cruciate ligamentdeficient knee. Arthroscopy: The Journal of Arthroscopic and Related Surgery, 2008, 24,1045-1051.

296. Bendjaballah MZ, Shirazi-Adl A, and Zukor DJ, Biomechanical response of the passive human knee joint under anterior-posterior forces. Clinical Biomechanics, 1998, 13,625-633.

297. Waite JC, Beard DJ, Dodd CAF, Murray DW, and Gill HS, In vivo kinematics of the acl-deficient limb during running and cutting. Knee Surgery, Sports Traumatology, and Arthroscopy, 2005, 13,377-384.

298. Millett PJ, Willis AA, and Warren RF, Associated injuries in pediatric and adolescent anterior cruciate tears: Does a delay in treatment increase the risk of meniscal tear? Arthroscopy: The Journal of Arthroscopic and Related Surgery, 2002, 18,955-959. 
299. Majewski M, Sabelt S and Steinbruck K, Epidemiology of athletic knee injuries: A 10-year study. The Knee, 2006, 13,3:184-188.

300. Radin EL, De Lamotte F and Maquet P, Role of the menisci in the distribution of stress in the knee. Clinical Orthopaedics and Related Research, 1984, 185:290-4.

301. Meyer EG and Haut RC, Excessive compression of the human tibio-femoral joint causes acl rupture. Journal of Biomechanics, Nov 2005, 38,11:2311-6.

302. Brown T, Johnston R, Saltzman C, et al., Posttraumatic osteoarthritis: A first estimate of incidence, prevalence, and burden of disease. Journal of Orthopaedic Trauma, Nov-Dec 2006, 20,10:739-744.

303. Roos H, Adalberth T, Dahlberg L, et al., Osteoarthritis of the knee after injury to the anterior cruciate ligament or meniscus: The influence of time and age. OsteoArthritis and Cartilage, 1995, 3,4:261-267.

304. Szczodry M, Coyle C, Kramer S, et al., Progressive chondrocyte death after impact injury indicates a need for chondroprotective therapy. American Journal of Sports Medicine, 2009, 37,12:2318-2322.

305. Kisiday J, Vanderploeg E, Mcilwraith C, et al., Mechanical injury of explants from the articulating surface of the inner meniscus. Archives of Biochemistry and Biophysics, 2010, 494,138-144.17 Nov 2009.

306. Ahmed AM, Mow V, Arnoczky S and Jackson D, The load bearing role of the knee menisci. 1992, In: Mow VC et al (eds), Knee meniscus basic and clinical foundations. New York: Raven Press, 59-73.

307. Boyd KT and Myers PT, Meniscus preservation; rationale, repair techniques and results. Knee, Mar 2003, 10,1:1-11.

308. Sniekers YH, Intema F, Lafeber FP, et al., A role for subchondral bone changes in the process of osteoarthritis; a micro-ct study of two canine models. BMC Musculoskeletal Disorders, 2008, 9,20.

309. Stoop R, Buma P, Van Der Kraan PM, et al., Type ii collagen degradation in articular cartilage fibrillation after anterior cruciate ligament transection in rats. OsteoArthritis and Cartilage, May 2001, 9,4:308-15. 
310. Setton LA, Elliott DM and Mow VC, Altered mechanics of cartilage with osteoarthritis: Human osteoarthritis and an experimental model of joint degeneration. OsteoArthritis and Cartilage, Jan 1999, 7,1:2-14.

311. Johnson DL, Urban WP, Caborn DNM, et al., Articular cartilage changes seen with magnetic resonance imaging-detected bone bruises associated with acute anterior cruciate ligament rupture. American Journal of Sports Medicine, 1998, 26,409-414.

312. Sarzi-Puttini P, Cimmino MA, Scarpa R, et al., Osteoarthritis: An overview of the disease and its treatment strategies. Seminars in Arthritis and Rheumatism, 2005, 35 1:1-10.

313. Meister K, Indelicato PA, Spanier S, et al., Histology of the torn meniscus: A comparison of histologic differences in meniscal tissue between tears in anterior cruciate ligament-intact and anterior cruciate ligament-deficient knees. American Journal of Sports Medicine, Sep 2004, 32,6:1479-83.

314. Daniel DM, Stone ML, Dobson BE, et al., Fate of the ACL-injured patient. A prospective outcome study. American Journal of Sports Medicine, Sep-Oct 1994, 22,5:632-44.

315. Oda H, Igarashi M, Sase HS, T, et al., Bone bruise in magnetic resonance imaging strongly correlates with the production of joint effusion and with knee osteoarthritis. Journal of Orthopaedic Science, 2008, 13,1:7-15.

316. Lepinski N, Killian M, Isaac R, et al., Characterizing lapine meniscal tissue: a regional comparison between normal medial and lateral menisci. 2009, Proceedings of the American Society of Mechanical Engineers Summer Bioengineering Conference, Lake Tahoe, CA.

317. Killian M, Lepinski N, Haut R, et al., Regional and zonal histo-morphological characteristics of the lapine menisci. Anatomical Record, 2010, In Press.

318. Killian M, Lepinski N, Isaac DI, et al., Regional glycosaminoglycan coverage of the healthy rabbit menisci. 2009, Proceedings of the American Society of Mechanical Engineers Summer Bioengineering Conference, Lake Tahoe, CA. 
319. Rolauffs B, Muehleman C, Li J, et al., Vulnerability of the superficial zone of immature articular cartilage to compressive injury. Arthritis and Rheumatism, 2010, Jun 15 , e-pub ahead of print.

320. Henninger $\mathrm{H}$, Underwood $\mathrm{CA}, \mathrm{Ga}$ and Weiss $\mathrm{J}$, Effect of sulfated glycosaminoglycan digestion on the transverse permeability of medial collateral ligament. Journal of Biomechanics, 2010, 43,13: 2567-2573.

321. Katta J, Stapleton T, Ingham E, et al., The effect of glycosaminoglycan depletion on the friction and deformation of articular cartilage. Proceedings of the Institution of Mechanical Engineers, Part H: Journal of Engineering in Medicine, 2008, 222,1:111.Jan.

322. Isaac DI, Meyer EG and Haut RC, Development of a traumatic anterior cruciate ligament and meniscal rupture model with a pilot in vivo study. Journal of Biomechanical Engineering, June 2010, 132,6: online.

323. Sonoda M, Harwood F, Amiel M, et al., The effects of hyaluronan on the meniscus in the anterior cruciate ligament-deficient knee. Journal of Orthopaedic Science, 2000, 5,2:157-164.

324. Sun Y, Mauerhan D, Honeycutt P, et al., Calcium deposition in osteoarthritic meniscus and meniscal cell culture. Arthritis Research and Therapy, 2010, 12,2010 Mar 30.

325. McGonagle D, Tan A, Carey J, et al., The anatomical basis for a novel classification of osteoarthritis and allied disorders. Journal of Anatomy, 2010, 216,3: online.

326. Umemoto $\mathrm{Y}$, Oka $\mathrm{T}$, Inoue $\mathrm{T}$, et al., Imaging of a rat osteoarthritis model using (18)f-fluoride positron emission tomography. Annals of Nuclear Medicine, 2010, 31 Aug 2010.

327. Hatayama K, Higuchi H, Kimura M, et al., Histologic changes after meniscal repair using radiofrequency energy in rabbits. Arthroscopy, 2007, 23,3:299-304.

328. Nishida M, Higuchi H, Kobayashi Y, et al., Histological and biochemical changes of experimental meniscus tear in the dog knee. Journal of Orthopaedic Science, 2005, $10,4: 406-413$ 
329. McCarty D, Crystal-induced inflammation of the joints. Annual Review of Medicine, 1970, 21,357-366.

330. Leroux MA, Arokoski J, Vail TP, et al., Simultaneous changes in the mechanical properties, quantitative collagen organization, and proteoglycan concentration of articular cartilage following canine meniscectomy. Journal of Orthopaedic Research, 2000, 18,3:383-92.

331. Armstrong CG and Mow VC, Variations in the intrinsic mechanical properties of human articular cartilage with age, degeneration, and water content. Journal of Bone and Joint Surgery: America, Jan 1982, 64,1:88-94.

332. Antoniou JS, Steffen T, Nelson F, Winterbottom N, et al., The human lumbar intervertebral disc: Evidence for changes in the biosynthesis and denaturation of the extracellular matrix with growth, maturation, ageing, and degeneration. Journal of Clinical Investigations, 1996, 98,996-1003.

333. Pearce R, Grimmer B and Adams M, Degeneration and the chemical composition of the human lumbar intervertebral disc. Journal of Orthopaedic Research, 1987, 5,198205.

334. Ahmed AM and Burke DL, In-vitro measurement of static pressure distribution in synovial joints--part I: Tibial surface of the knee. Journal of Biomechanical Engineering, 1983, 105,3:216-225.

335. Brown TD and Shaw DT, In vitro contact stress distribution on the femoral condyles. Journal of Orthopaedic Research, 1984, 2,2:190-9.

336. Jones HP, Appleyard RC, Mahajan S, et al., Meniscal and chondral loss in the anterior cruciate ligament injured knee. Sports Medicine, 2003, 33,14:1075-1089.

337. Mandalia V and Henson JH, Traumatic bone bruising--a review article. European Journal of Radiology, Jul 2008, 67,1:54-61.

338. Meyer EG, Baumer TG, Slade JM, et al., Tibiofemoral contact pressures and osteochondral microtrauma during anterior cruciate ligament rupture due to excessive compressive loading and internal torque of the human knee. American Journal of Sports Medicine, Oct 2008, 36,10:1966-77. 
339. Lotz M, Posttraumatic osteoarthritis: Pathogenesis and pharmacological treatment options. Arthritis Research and Therapy, 2010, 12,211: online.

340. Isaac D, Golenberg $\mathrm{N}$ and Haut $\mathrm{R}$, Acute repair of chondrocytes in the rabbit tibiofemoral joint following blunt impact using p188 surfactant and a preliminary investigation of its long-term efficacy. Journal of Orthopaedic Research, 2010, 28,4:553-8.

341. Selinummi J, Seppälä J, Yli-Harja O, et al., Software for quantification of labeled bacteria from digital microscope images by automated image analysis. BioTechniques, 2005, 39,6:859-863.

342. Torzilli PA, Grigiene R, Borrelli J, Jr., et al., Effect of impact load on articular cartilage: Cell metabolism and viability, and matrix water content. Journal of Biomechanical Engineering, Oct 1999, 121,5:433-41.

343. Lawrence RC, Felson DT, Helmick CG, et al., Estimates of the prevalence of arthritis and other rheumatic conditions in the United States, part II. Arthritis and Rheumatism, 2008, 58,1:26-35.

344. Felson DT, Lawrence RC, Dieppe PA, et al., Osteoarthritis: New insights. Part 1: The disease and its risk factors. Annals of Internal Medicine, Oct 17 2000, 133,8:63546.

345. Kessler M, Behrend H and Henz SS, G, Function, osteoarthritis and activity after acl-rupture: 11 years follow-up results of conservative versus reconstructive treatment. Knee Surgery, Sports Traumatology, and Arthroscopy, 2008, 16,442-448.

346. D'lima DD, Hashimoto S, Chen PC, et al., Impact of mechanical trauma on matrix and cells. Clinical Orthopaedics, Oct 2001, 391 Suppl:S90-9.

347. Clements KM, Bee ZC, Crossingham GV, et al., How severe must repetitive loading be to kill chondrocytes in articular cartilage? OsteoArthritis and Cartilage, Jul 2001, 9,5:499-507.

348. Wyllie A, Apoptosis: An overview. British Medical Bulletin, 1997, 53,3:451-465.

349. Clockaert S, Bastiaansen-Jenniskens YM, Runhaar J, et al., The infrapatellar fat pad should be considered as an active osteoarthritic joint tissue: A narrative review. OsteoArthritis and Cartilage, 2010, 18, 876-882. 
350. Benjamin $\mathrm{M}$ and Mcgonagle $\mathrm{D}$, Histopathologic changes at "synovio-entheseal complexes" suggesting a novel mechanism for synovitis in osteoarthritis and spondylarthritis. Arthritis and Rheumatism, 2007, 56,11:3601-3609.

351. Walsh D, Bonnet C, Turner E, et al., Angiogenesis in the synovium and at the osteochondral junction in osteoarthritis. OsteoArthritis and Cartilage, 2007, 15,743751.

352. Schlaak J, Pfers I, Büschenfelde KMZ, et al., Different cytokine profiles in the synovial fluid of patients with osteoarthritis, rheumatoid arthritis and seronegative spondylarthropathies. Clinical and Experimental Rheumatology, 1996, 14,2:155-162.

353. Alvarez-Soria M, Largo R, Santillana J, et al., Long term nsaid treatment inhibits COX-2 synthesis in the knee synovial membrane of patients with osteoarthritis: Differential proinflammatory cytokine profile between celecoxib and aceclofenac. Annals of Rheumatic Diseases, 2006, 65,8:998-1005.

354. Laine L, Curtis S, Langman M, et al., Lower gastrointestinal events in a doubleblind trial of the cyclo-oxygenase-2 selective inhibitor etoricoxib and the traditional nonsteroidal anti-inflammatory drug diclofenac. Gastroenterology, 2008, 135,5:15171525.

355. Paul H, Reginato A and Schumacher H, Alizarin red s staining as a screening test to detect calcium compounds in synovial fluid. Arthritis \& Rheumatism, 1983, 26,2:191-200. 
Appendix A: Data 
Table A.1. Quantitative PCR data for porcine meniscal explants treated with and without IL-1RA subjected to $2 \mathrm{hrs}$ of dynamic compression exercises. In: Research folder, IL1RA Research subfolder, "IL1-RA Compiled Results_Updatedv2.xlsx"

\begin{tabular}{|c|c|c|c|c|c|c|c|c|}
\hline & \multicolumn{3}{|c|}{ IL-1RA Treated } & \multicolumn{3}{|c|}{ Control } \\
\hline & & & $0 \%$ & $10 \%$ & $20 \%$ & $0 \%$ & $10 \%$ & $20 \%$ \\
\hline \multirow{4}{*}{ ADAMTS4 } & \multirow{2}{*}{ Sup } & Average & 0.47 & 0.15 & 0.34 & 1.95 & 0.81 & 1.96 \\
\hline & & StError & 0.19 & 0.08 & 0.22 & 0.54 & 0.21 & 0.50 \\
\hline & \multirow{2}{*}{ Deep } & Average & 0.61 & 0.40 & 0.53 & 1.86 & 0.74 & 2.55 \\
\hline & & StError & 0.26 & 0.18 & 0.09 & 1.20 & 0.41 & 1.16 \\
\hline \multirow{4}{*}{ ADAMTS5 } & \multirow{2}{*}{ Sup } & Average & 3.16 & ND & 24.45 & 2.77 & 25.09 & 27.23 \\
\hline & & StError & 0.84 & ND & 9.24 & 0.68 & 14.67 & 13.31 \\
\hline & \multirow{2}{*}{ Deep } & Average & 1.16 & 2.07 & 3.20 & 1.70 & 10.81 & 1.63 \\
\hline & & StError & 0.53 & 0.72 & 1.11 & 0.54 & 3.61 & 0.21 \\
\hline \multirow{4}{*}{ IL-1 $\alpha$} & \multirow{2}{*}{ Sup } & Average & 3.65 & 3.73 & 0.50 & 3.07 & 6.86 & 6.16 \\
\hline & & StError & 1.52 & 2.47 & 0.22 & 0.35 & 2.31 & 1.09 \\
\hline & \multirow{2}{*}{ Deep } & Average & 1.10 & 0.73 & 0.94 & 4.28 & 5.73 & 6.11 \\
\hline & & StError & 0.33 & 0.51 & 0.69 & 0.73 & 2.01 & 0.49 \\
\hline \multirow{2}{*}{ Aggrecan } & \multirow{2}{*}{ Sup } & Average & 10.64 & 17.37 & 28.65 & 11.41 & 18.00 & 22.75 \\
\hline & & StError & 9.17 & 8.99 & 13.67 & 1.97 & 4.34 & 8.08 \\
\hline \multirow{4}{*}{ COX-2 } & \multirow{2}{*}{ Sup } & Average & 8.83 & 7.48 & 1.30 & 1.54 & 4.23 & 2.28 \\
\hline & & StError & 4.79 & 5.26 & 0.37 & 0.37 & 1.67 & 0.42 \\
\hline & \multirow{2}{*}{ Deep } & Average & 2.93 & 1.32 & 2.03 & 1.43 & 4.00 & 2.58 \\
\hline & & StError & 2.06 & 0.33 & 1.34 & 0.24 & 1.17 & 0.69 \\
\hline \multirow{4}{*}{ iNOS } & \multirow{2}{*}{ Sup } & Average & 0.32 & 0.48 & 0.50 & 0.53 & 0.73 & 1.29 \\
\hline & & StError & 0.09 & 0.11 & 0.11 & 0.11 & 0.14 & 0.37 \\
\hline & \multirow{2}{*}{ Deep } & Average & 0.30 & 0.48 & 0.27 & 0.27 & 0.43 & 0.66 \\
\hline & & StError & 0.10 & 0.21 & 0.01 & 0.06 & 0.10 & 0.18 \\
\hline
\end{tabular}


Table A.2. Control lapine meniscal histomorphometric measurements. $n=5$ for each group. In: Research folder, Rabbit Histo subfolder, "ACLT, TEAR, and control data_v1.xlsx"

\begin{tabular}{ll|c|c|c|c|c|c|} 
& \multicolumn{2}{c}{ MA } & \multicolumn{1}{c}{ MC } & MP & \multicolumn{1}{c}{ LA } & \multicolumn{1}{c}{ LC } \\
\cline { 2 - 8 } Tissue Area & average & $\mathbf{4 . 7 4}$ & $\mathbf{2 . 7 6}$ & $\mathbf{3 . 5 2}$ & $\mathbf{5 . 5 7}$ & $\mathbf{3 . 9 3}$ & $\mathbf{5 . 1 6}$ \\
$\mathrm{mm}^{2}$ & stderror & 0.47 & 0.43 & 0.31 & 0.62 & 0.38 & 0.40 \\
\cline { 3 - 9 } Cell Density & average & $\mathbf{4 2 5 . 4 5}$ & $\mathbf{3 9 4 . 2 8}$ & $\mathbf{4 3 1 . 1 8}$ & $\mathbf{4 6 2 . 7 6}$ & $\mathbf{3 9 4 . 0 0}$ & $\mathbf{5 6 9 . 6 2}$ \\
Cells $/ \mathrm{mm}^{2}$ & stderror & 29.55 & 48.34 & 30.12 & 22.05 & 25.11 & 25.75 \\
\cline { 3 - 8 } GAG Coverage & average & $\mathbf{2 4 . 4 4}$ & $\mathbf{5 . 4 1}$ & $\mathbf{4 . 3 5}$ & $\mathbf{1 3 . 5 6}$ & $\mathbf{1 4 . 1 9}$ & $\mathbf{1 2 . 3 0}$ \\
\% area coverage & stderror & 8.65 & 1.70 & 2.29 & 3.48 & 4.19 & 3.75 \\
\cline { 3 - 8 } & & & & & & &
\end{tabular}

Table A.3. Tissue area of histological sections of control, impacted (TEAR), ACLT, and ACLT sham (sham) medial (M) and lateral (L) menisci in anterior (A), central (C), and posterior (P) regions. In: Research folder, Rabbit Histo subfolder, "ACLT, TEAR, and control data_v1.xlsx"

\begin{tabular}{|l|l|c|c|c|c|}
\multicolumn{2}{c}{ Control } & TEAR & ACLT & Sham \\
\hline MA & average & $\mathbf{4 . 7 4}$ & $\mathbf{4 . 3 3}$ & $\mathbf{1 1 . 0 9}$ & $\mathbf{4 . 6 0}$ \\
& stderror & 0.47 & 1.45 & 2.39 & 0.78 \\
\hline MC & average & $\mathbf{2 . 7 6}$ & $\mathbf{3 . 1 3}$ & $\mathbf{6 . 5 5}$ & $\mathbf{2 . 7 6}$ \\
& stderror & 0.43 & 1.39 & 2.92 & 0.55 \\
\hline MP & average & $\mathbf{3 . 5 2}$ & $\mathbf{4 . 2 5}$ & $\mathbf{6 . 5 8}$ & $\mathbf{3 . 7 4}$ \\
& stderror & 0.31 & 0.27 & 3.13 & 0.50 \\
\hline LA & average & $\mathbf{5 . 5 7}$ & $\mathbf{4 . 1 2}$ & $\mathbf{8 . 4 5}$ & $\mathbf{5 . 2 2}$ \\
& stderror & 0.62 & 0.72 & 1.39 & 0.52 \\
\hline LC & average & $\mathbf{3 . 9 3}$ & $\mathbf{4 . 7 0}$ & $\mathbf{7 . 0 6}$ & $\mathbf{4 . 8 9}$ \\
& stderror & 0.38 & 0.44 & 3.26 & 0.62 \\
\hline \multirow{2}{*}{$\mathbf{P}$} & average & $\mathbf{5 . 1 6}$ & $\mathbf{3 . 0 3}$ & $\mathbf{1 1 . 8 3}$ & $\mathbf{4 . 2 1}$ \\
& stderror & 0.40 & 0.09 & 0.18 & 0.45 \\
\hline
\end{tabular}


Table A.4. Cell density of histological sections of control, impacted (TEAR), ACLT, and ACLT sham (sham) medial (M) and lateral (L) menisci in anterior (A), central (C), and posterior (P) regions. In: Research folder, Rabbit Histo subfolder, "ACLT, TEAR, and control data_v1.xlsx"

\begin{tabular}{|c|c|c|c|c|c|}
\hline & & Control & TEAR & ACLT & Sham \\
\hline \multirow[t]{2}{*}{ MA } & average & 425.45 & 616.25 & 426.57 & 533.55 \\
\hline & stderror & 29.55 & 27.50 & 93.65 & 34.27 \\
\hline \multirow[t]{2}{*}{ MC } & average & 394.28 & 364.14 & 479.39 & 604.27 \\
\hline & stderror & 48.34 & 118.16 & 87.07 & 25.15 \\
\hline \multirow[t]{2}{*}{ MP } & average & 431.18 & 622.23 & 542.90 & 670.08 \\
\hline & stderror & 30.12 & 21.05 & 120.52 & 51.48 \\
\hline \multirow[t]{2}{*}{$\mathbf{L A}$} & average & 462.76 & 669.33 & 472.07 & 551.62 \\
\hline & stderror & 22.05 & 84.92 & 18.30 & 72.19 \\
\hline \multirow[t]{2}{*}{$\mathrm{LC}$} & average & 394.00 & 533.18 & 592.36 & 547.35 \\
\hline & stderror & 25.11 & 32.79 & 153.52 & 19.08 \\
\hline \multirow[t]{2}{*}{$\mathbf{L P}$} & average & 569.62 & 553.56 & 383.24 & 571.66 \\
\hline & stderror & 25.75 & 71.83 & 19.27 & 23.73 \\
\hline
\end{tabular}

Table A.5. Percentage of area covered by sulfated GAG (positive SafO stain) for histological sections of control, ACLT sham (SHAM), impacted (TEAR), and ACLT medial $(\mathrm{M})$ and lateral $(\mathrm{L})$ menisci in anterior $(\mathrm{A})$, central $(\mathrm{C})$, and posterior $(\mathrm{P})$ regions. In: Research folder, Rabbit Histo subfolder, "ACLT, TEAR, and control data_v1.xlsx"

\begin{tabular}{|c|c|c|c|c|c|}
\hline \multirow{3}{*}{ MA } & & Control & TEAR & ACLT & Sham \\
\hline & average & 16.97 & 5.13 & 6.23 & 6.50 \\
\hline & stderror & 4.84 & 4.97 & 2.89 & 2.32 \\
\hline \multirow[t]{2}{*}{ MC } & average & 6.25 & 0.54 & 1.07 & 5.55 \\
\hline & stderror & 2.11 & 0.28 & 0.36 & 2.41 \\
\hline \multirow[t]{2}{*}{ MP } & average & 4.09 & 0.70 & 1.94 & 2.30 \\
\hline & stderror & 1.96 & 0.48 & 1.61 & 0.76 \\
\hline \multirow[t]{2}{*}{ LA } & average & 12.77 & 8.63 & 1.47 & 4.47 \\
\hline & stderror & 4.54 & 5.29 & 0.65 & 1.56 \\
\hline \multirow[t]{2}{*}{$\mathbf{L C}$} & average & 15.86 & 8.71 & 0.42 & 2.04 \\
\hline & stderror & 5.22 & 4.23 & 0.07 & 0.63 \\
\hline \multirow[t]{2}{*}{$\mathbf{L P}$} & average & 13.32 & 9.94 & 0.43 & 5.76 \\
\hline & stderror & 5.13 & 5.46 & 0.34 & 3.09 \\
\hline
\end{tabular}


Table A-6. Impaction inventory of rabbits housed at Michigan Tech.

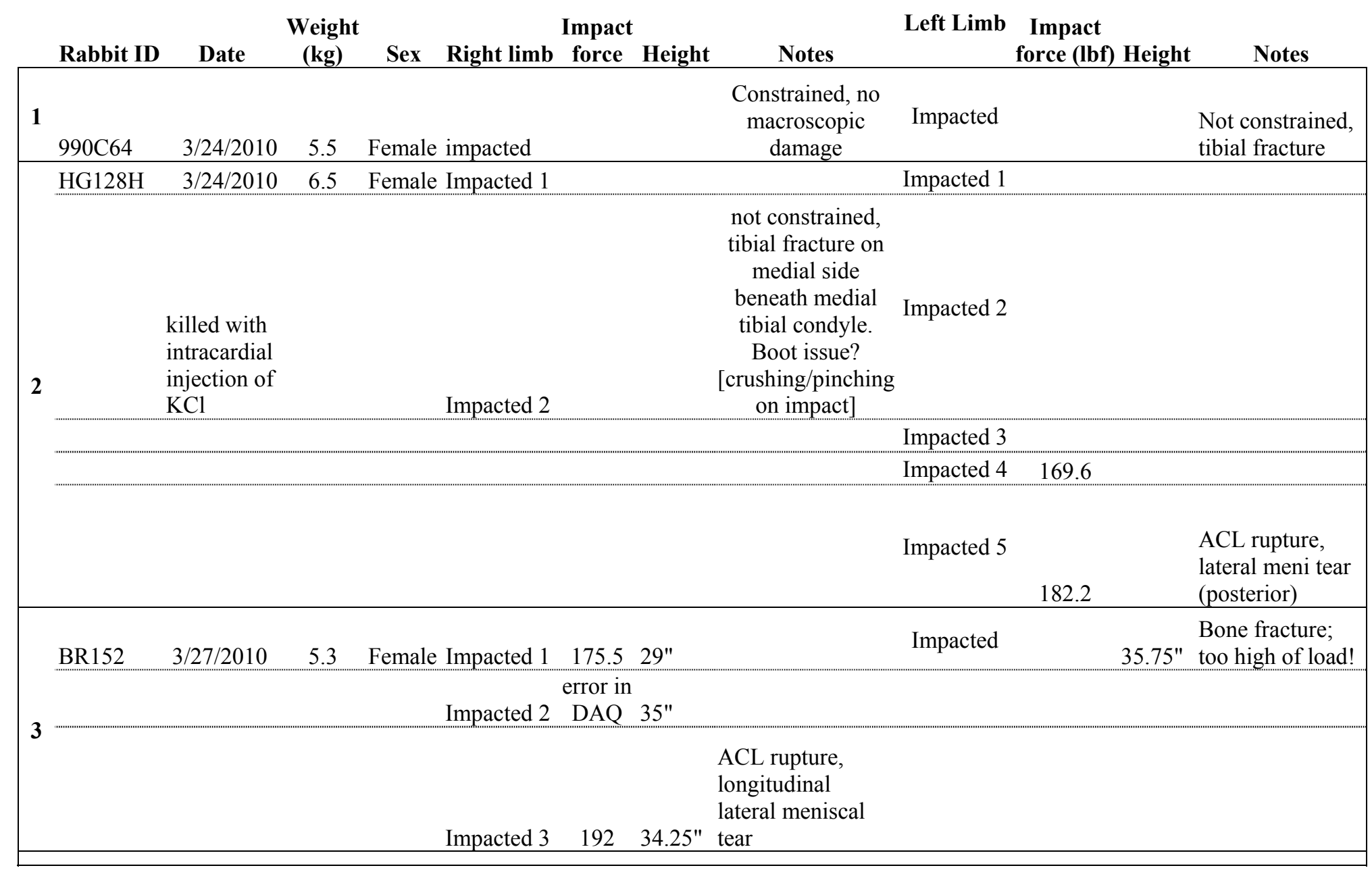




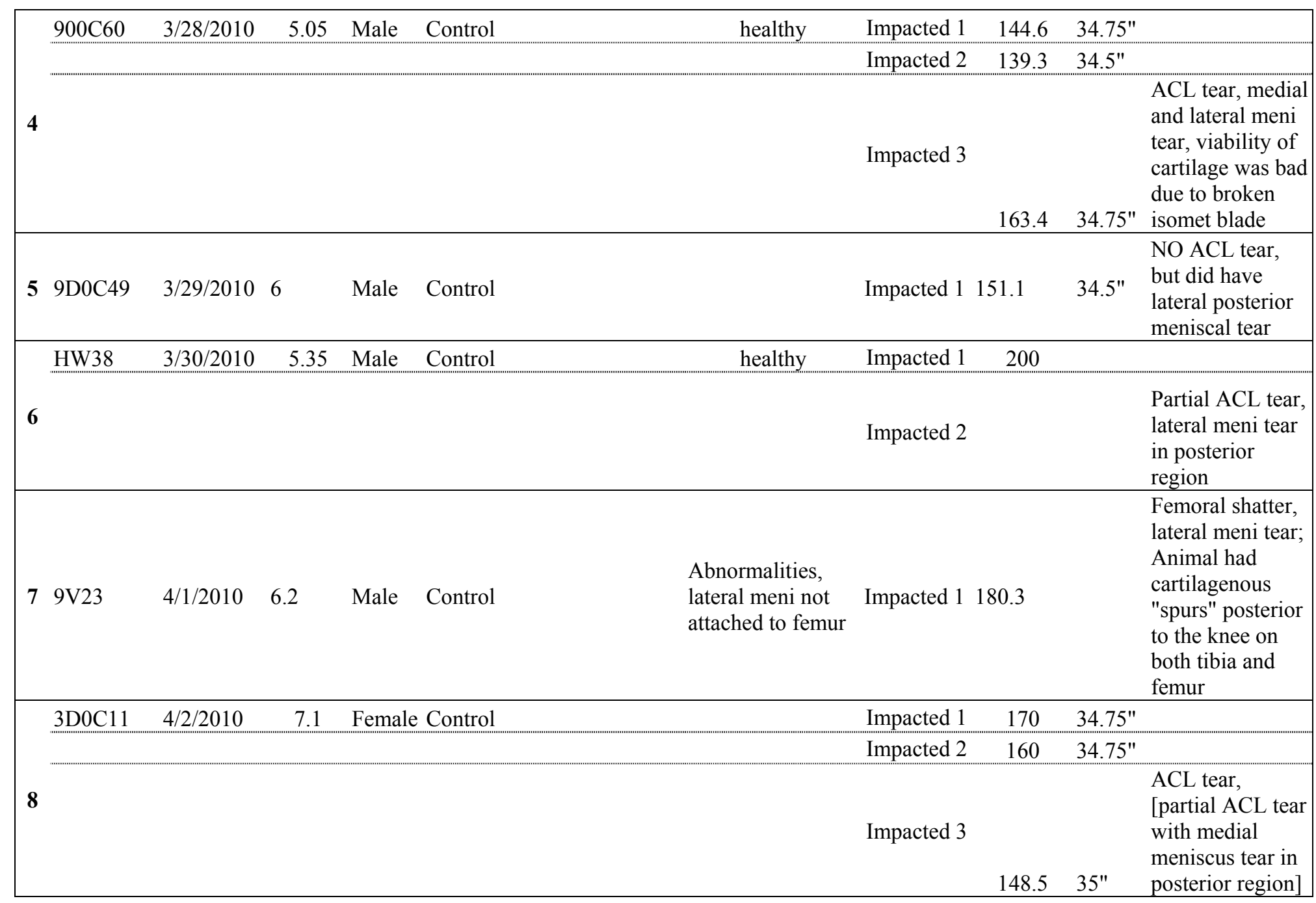




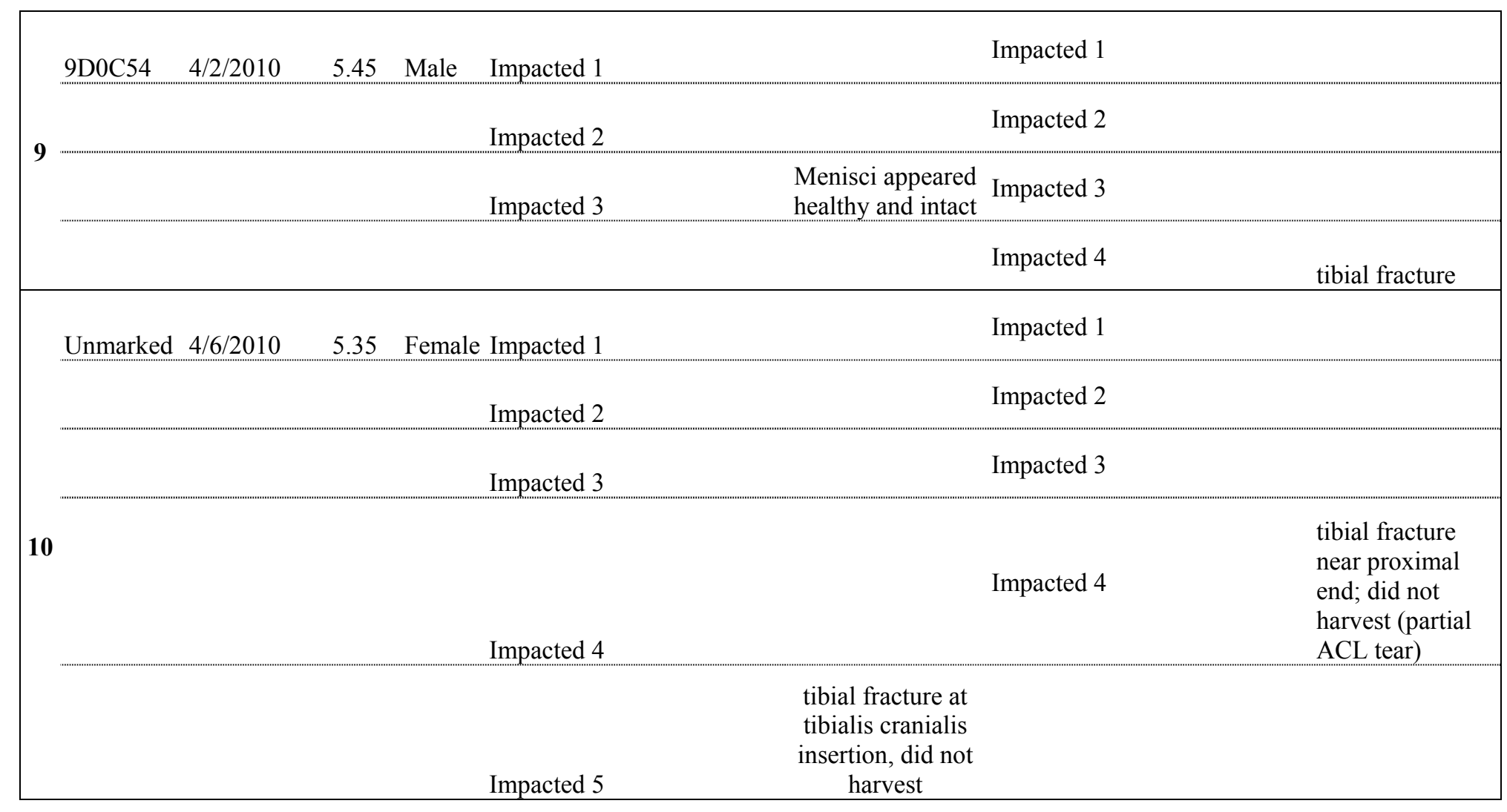


Appendix B: Protocols 


\section{Decalcification and embedding: Histology prep protocol}

Protocol originally written by: Meghan McGee; Modified by Megan Killian

Time to Complete: 5-10 days for decalcification, 60+ minutes for slide prep (depending on number of samples you want to prepare)

\begin{tabular}{|c|c|c|c|}
\hline Supplies Required & $\begin{array}{l}\text { Supplier and } \\
\text { Catalog } \\
\text { Number }\end{array}$ & $\begin{array}{l}\text { Storage } \\
\text { Conditions }\end{array}$ & Location \\
\hline Gloves & $\begin{array}{l}\text { Fisher, 19-050- } \\
221 \mathrm{~B} \quad \text { (and } \\
221 \mathrm{C}, 221 \mathrm{D})\end{array}$ & --- & Portage wall \\
\hline Tetrasodium EDTA & $\begin{array}{l}\text { Sigma-Aldrich } \\
\text { E6511 }\end{array}$ & Sealed & $\begin{array}{l}\text { Chemical cabinet by } \\
\text { Instron }\end{array}$ \\
\hline Glacial acetic acid & Chem Stores & Sealed & $\begin{array}{l}\text { Chemical cabinet by } \\
\text { Instron }\end{array}$ \\
\hline Pipette \& tips & & & Portage wall \\
\hline Electronic balance & & Clean & Chalkboard wall \\
\hline $\begin{array}{l}\text { Sucrose (white } \\
\text { granulated sugar) }\end{array}$ & Walmart & Sealed & Portage wall \\
\hline $\begin{array}{l}\text { Embedding medium: } \\
\text { Tissue-Tek }{ }^{\circledR} \text { O.C.T. }\end{array}$ & $\begin{array}{l}\text { VWR, 25608- } \\
930\end{array}$ & $\begin{array}{l}\text { Tightly } \\
\text { capped }\end{array}$ & $\begin{array}{l}\text { Portage wall or by } \\
\text { centrifuge by cryostat }\end{array}$ \\
\hline Disposable Base Molds & $\begin{array}{l}\text { Fisher, 22- } \\
038217\end{array}$ & --- & $\begin{array}{l}\text { Jeremy Goldman's } \\
\text { Lab }\end{array}$ \\
\hline $\begin{array}{lr}\text { Tissue } & \text { Path High } \\
\text { Profile } & \text { Microtome } \\
\text { blades } & \\
\end{array}$ & $\begin{array}{l}\text { Fisher Sci prod } \\
\# \text { 22-244-028 }\end{array}$ & $\begin{array}{l}\text { Inside } \\
\text { included } \\
\text { container }\end{array}$ & Cryostat \\
\hline Liquid nitrogen & Chem Store & Inside dewar & Chem Store \\
\hline $\begin{array}{l}\text { Slide glass (coated in } \\
\text { gelatin- } \\
\text { chromagnesium) }\end{array}$ & $\begin{array}{l}\text { Large: } \\
\text { Cat Nisher } \\
\text { 550A } \\
\text { Small: } \\
\text { Cat No. } 48300- \\
025\end{array}$ & --- & Histology drawer \\
\hline Cover slips & $\begin{array}{lr}\text { Large: } & \text { Fisher } \\
\text { Cat No. } & 12- \\
545 \mathrm{H} & \\
\text { Small: } & \text { Fisher } \\
\text { Cat No. } & 12- \\
545 \mathrm{~B} & \end{array}$ & --- & Histology drawer \\
\hline Old (broken) slide glass & --- & --- & $\begin{array}{lr}\text { Slide } & \text { preparation } \\
\text { drawer in histology } \\
\text { room }\end{array}$ \\
\hline
\end{tabular}




\section{Preparation and Decalcification}

1. Remove meniscus from tibial plateau using appropriate techniques in order to maintain orientation and integrity of the tissue you wish to use. If sectioning meniscal body, use scalpel to separate meniscus at attachments and synovium from tibial plateau. Thoroughly remove soft tissue (including the synovium) with a scalpel

a. If performing thickness measurements, do this step now

b. If using only the meniscus body and not attachments, skip to step 11 .

For attachments only!

2. If sectioning attachments, use diamond-blade bone saw in $\mathrm{Rm} 1006$ to get bone block and do not cut meniscus.

3. Section the meniscal attachment longitudinally (parallel w.r.t. collagen fibers of ligament) with a bandsaw to isolate separate sections for use in SEM and histology (or for regional comparisons between proximal and distal). Clean off debris/visible marrow with a water jet, pat dry, and obtain the starting mass of the sample with the scale.

4. Mix a batch of $14 \%$ tetrasodium EDTA. Store in a closeable container (e.g., old PBS or FBS bottle) under the fume hood.

a. \% is by weight: $500 \mathrm{ml}$ of a $14 \%$ EDTA solution contains $70 \mathrm{~g}$ of EDTA and $500 \mathrm{ml}$ of deionized, distilled water (use deionized distilled water especially for samples to be used in IHC)

5. Check the $\mathrm{pH}$ of the EDTA solution with a $\mathrm{pH}$ meter; the initial $\mathrm{pH}$ will be approximately 10.5 . Adjust the $\mathrm{pH}$ down to 7.6 by adding small amounts (1-3ml at a time) of glacial acetic acid with a pipette. Check the $\mathrm{pH}$ after each addition, and stop when the $\mathrm{pH}$ is approximately 7.6.

6. Pour a small amount of the EDTA solution into a glass beaker ( $\sim 50 \mathrm{ml}$ for $2-3$ bones)

7. Place each meniscal sample in some sort of a water-permeable container so that each sample can be clearly identified and distinguished from one another (e.g., close inside a tissue cassette for small, cloth bag for larger samples).

8. Submerge the sample(s) in the EDTA solution and cover with parafilm. Place beaker under the fume hood for 24 hours.

9. After 24 hours have passed, remove the sample from the EDTA with forceps, pat dry, and obtain mass. Resubmerge sample in fresh EDTA; dispose of used EDTA down the sink drain.

10. Repeat steps 8 and 9 until sample no longer loses mass in the 24 hour period and begins to GAIN mass instead 
a. mass is lost as EDTA chelates the mineral from the bone; mass will start to INCREASE when water begins to enter the tissue after decalcification is complete. This will likely take 3-10 days.

\section{For attachments AND Meniscal bodies}

11. When sample displays an increase in mass relative to its last mass reading, rinse it in distilled water and submerge the sample in a 1:1 mixture of O.C.T. and $30 \%$ sucrose. This can be done in a small beaker, or in one well of a 6 or 24-well plate (use the smallest well size possible to avoid wasting medium). Place the container in a vacuum desiccator (without desiccant) and turn on vacuum.

a. $30 \%$ sucrose $=75 \mathrm{~g}$ sucrose (white granulated table sugar) to $250 \mathrm{ml}$ distilled water

b. O.C.T. $=$ Tissue-Tek ${ }^{\circledR}$ O.C.T. (Optimal Cutting Temperature) Compound NOTE: DO NOT change O.C.T. for another cutting medium like Neg-50 cryomedia; they do not have comparable results, and O.C.T. has consistently worked well for bone and meniscal histology.

Leave samples in sucrose/O.C.T. mixture under vacuum for $\sim 3$ days to allow the sucrose and OCT to penetrate the collagen matrix. Do not let the samples become uncovered by medium at any time (check samples daily and add more sucrose/O.C.T. as needed). The samples can be left in sucrose/O.C.T. mixture longer than 3 days if needed or if samples are thick.

\section{Embedding / Freezing / Sectioning}

1. Obtain a disposable base mold (Fisher TissuePath disposable base mold, $15 \mathrm{x} 15 \mathrm{x}$ $5 \mathrm{~mm}$ ). Fill the indent in the mold with embedding medium (note: embedding medium is not the sucrose/OCT mixture used for fixation of tissue. It is just O.C.T., straight from the bottle)

2. Place the decalcified sample in the filled indent, making sure that the specimen does not protrude (much) above the opening

- Orient the specimen such that the bottom surface of the mold is the cutting surface for cryosectioning

3. Cover the specimen with the embedding medium - make sure the entire specimen is covered

4. Grip the edge of the mold with hemastats and lower the mold into a Styrofoam dish filled with liquid nitrogen. Leave the mold in the liquid nitrogen until the medium is completely frozen (usually when the liquid nitrogen no longer appears to be "boiling"). 
5. Remove the mold from the liquid nitrogen and use forceps to pop the embedded specimen out of the mold. Immediately set the embedded specimen inside the microtome so it will stay frozen or store samples in $-20^{\circ} \mathrm{C}$ until ready to use.

\section{Using the Cryostat}

6. Inside the microtome, place the gold chuck (grooved platform) in one of the first four holes in the "fast freeze rail" ( 2 columns of 6 holes each located on the far left inside the microtome). The slots are numbered - the first four slots are exposed to the lowest temperatures, and are located at the bottom of the fast freeze rail. Wait several minutes until the chuck is cold.

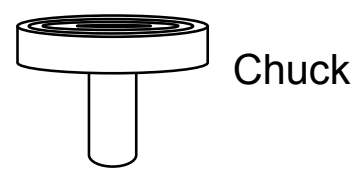

7. Remove a blade from the blade container and grip it by the flat edge. Raise the clamp on the right hand side of the blade holder and slide the blade in along the left hand side of the blade holder. If it won't go in, push gently on the bottom edge of the blade holder (the side closest to you). Push the clamp back up to lock the blade in place.

8. Wearing gloves, remove the chuck and hold the post in your hand, letting it warm up slightly. Spread embedding medium on the face of the chuck (over the grooved surface, making sure it goes down into the grooves), and put the chuck back in the fast freeze rail hole. NOTE: if the chuck is too cold when you put the medium on the grooved face, the medium will not go down into the grooves. You will have to pop off the frozen medium, wait for the chuck to warm up a bit more, and try again.

9. As soon as crystals start to appear on the outside edges of the embedding medium on the chuck face (this will happen quickly) push your embedded specimen into the center. It is best if you push the sample in flat side first (i.e., the side that was against the bottom of the mold) because this allows for a more even cut with the microtome blade.

10. Allow the entire chuck/specimen combination to freeze inside the cryostat, and then insert the post of the chuck into the black chuck holder. The black lever on the right secures the chuck in the holder, and the black knob on the left allows the chuck to rotate once it has been loosened. 


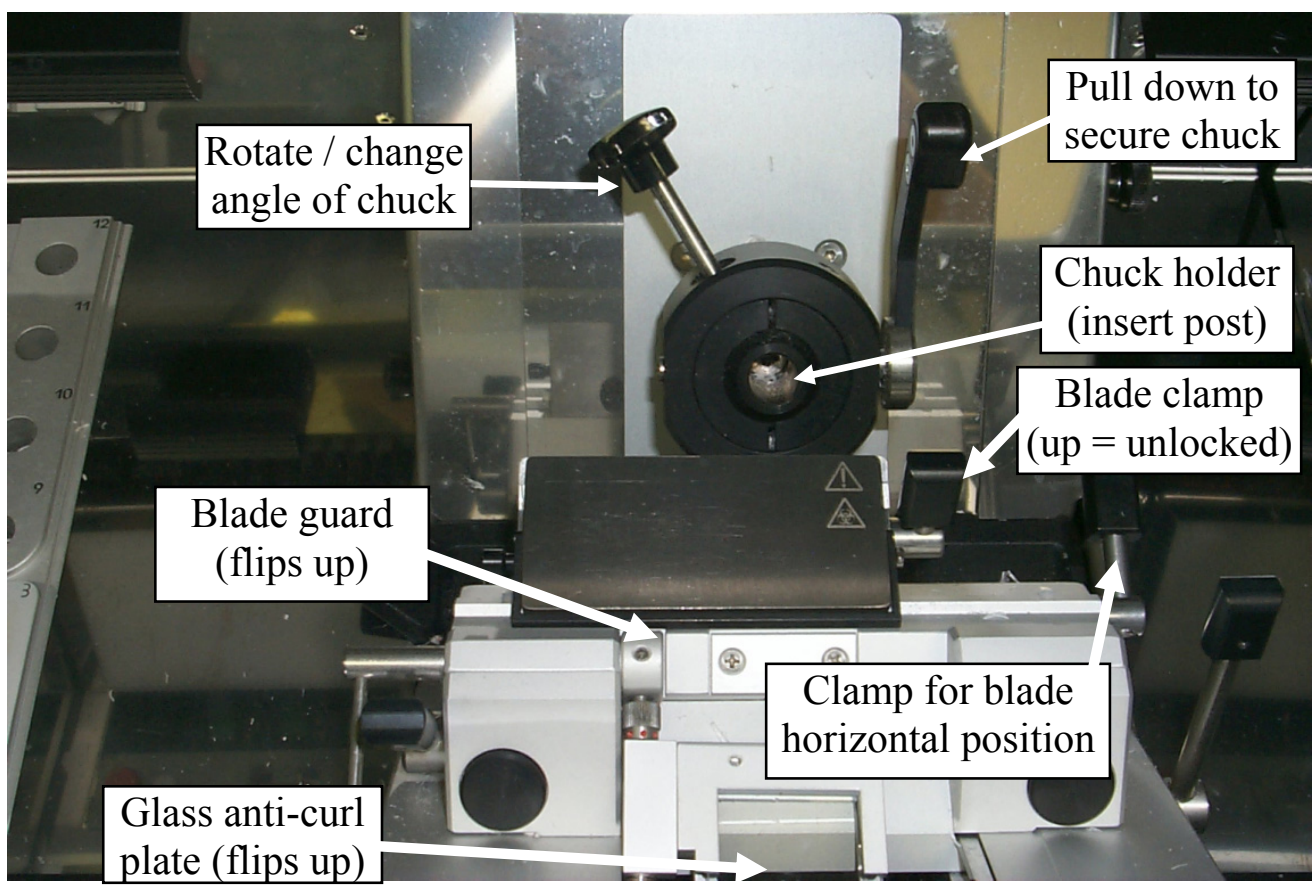

11. Adjust the vertical position of the sample with the handwheel so that it is centered vertically with respect to the blade.

12. Push the "up" arrow (blue, along the left hand side of the microtome, NOT next to the display) and hold it, and the specimen holder will move all the way back. Adjust the angle of the chuck face so that the specimen will cut well longitudinally. MAKE SURE THE BLADE GUARD IS UP when you do this. Once adjusted, push the "ACA" button, which will automatically advance the specimen to the blade.

13. Set the cryostat to trim (60 works well, but do not exceed 100) by pushing the middle button on the left side of the microtome's button panel next to the display (see below). This button will switch between trim and fine. The depth of the trim can be adjusted with the arrow buttons directly below the switch button.

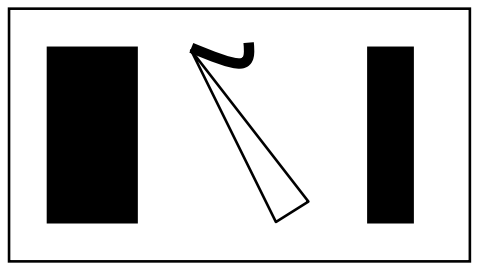

\section{Button to switch} between trim \& fine

14. Using the handwheel on the right hand side of the cryostat, raise and lower the embedded sample to trim through the frozen medium until you start to see the bone appear. Continue trimming until a good longitudinal cross-section appears. 
Adjust the angle of the chuck if the sample appears to be cutting unevenly (i.e., if sections appear to be thicker on one side, if they appear angled, etc.). MAKE SURE THE BLADE GUARD IS UP when you do this.

15. Flip the "glass anti-curl plate" up so that any sections cut will slide into it underneath the glass; this will prevent sections from curling.

16. Push the button (in Step 13) to switch to "Fine" instead of "Trim". Set the "Fine" depth to 10 um (using the arrow buttons) and begin cutting sections. If $10 \mathrm{um}$ does not yield good sections, increase the "fine" depth until good sections are obtained. Raise the glass plate periodically to clean out any "junk sections" with the brush kept inside the microtome.

17. When your first good (even thickness, longitudinal appearance) section is cut, transfer it to a piece of slide glass by raising the anti-curl plate and pressing a piece of slide glass against the specimen. Cut at least 2 more sections that you'll dispose of before the next section you plan to transfer to a slide (so that they'll be separated through the depth of the core). Do not place more than 3 sections per slide or the cover glass will not fit. Be careful of their placement when transferring to the slide glass - you won't be able to move them once they've been stuck to the slide, so make sure they're close enough together so that one coverglass will cover all 3. Obtain a total of 6 sections (2-3 per slide; 2-3 slides) per core. The remaining specimen can be refrozen or disposed of.

18. Remove the blade from the cryostat; raise the blade clamp to unlock it and use forceps to gently push along the right hand side of the blade, pushing the blade out the left side of the holder. Place the used blade in the disposal side of the blade container.

19. Push the "Menu" button to the right of the display panel and scroll down using the arrow keys to the left of the display panel until you reach the "ILLUM" option. Select $(\leftarrow)$ "Off" to turn the light inside the microtome off. Clean out any remaining junk by pushing it down the garbage chute in the bottom of the cryostat.

20. Lay slides on a flat surface.

21. Using a spray bottle, spritz $60^{\circ} \mathrm{C}$ deionized distilled water on slides to remove bubbles and promote the section adherence to the slide. Do not dip slides in water or saturate the slides. Cover slides with paper towels without touching the slides. Allow to dry overnight. 


\section{Safranin $\mathrm{O}$ staining protocol}

Time Period: 75 minutes

\section{EQUIPMENT LIST:}

Prepared slides (prepared in the slide preparation protocol)

Slide holder with handle

Tupperware

Distilled water

Xylene

Ethanol: $100 \%, 95 \%, 80 \%$

Weigerts iron hematoxylin working solution: mix equal portions of $\mathrm{A}$ and $\mathrm{B}$.

A $-5 \mathrm{~g}$ hematoxylin in $500 \mathrm{~mL} 95 \%$ ethanol

B - 20mL of $29 \%$ ferric chloride solution (5.8g ferric chloride in $20 \mathrm{~mL}$ distilled water), $475 \mathrm{~mL}$ distilled water, $5 \mathrm{~mL}$ concentrated $\mathrm{HCl}$

Tap water

Fast green FCF solution (0.1g fast green FCF in $1000 \mathrm{~mL}$ water)

$1 \%$ acetic acid solution $(10 \mathrm{~mL}$ glacial acetic acid and $990 \mathrm{~mL}$ distilled water)

$0.1 \%$ safranin $\mathrm{O}$ solution (1g safranin $\mathrm{O}$ in $1000 \mathrm{~mL}$ distilled water)

\section{PROCEDURE:}

Place the slides in the slide holder.

2. Stain with Weigert's iron hematoxylin working solution for 10 minutes.

3. Wash in running tap water for 10 minutes.

4. Stain with fast green (FCF) solution for 5 minutes.

5. Rinse quickly with $1 \%$ acetic acid solution for no more than $10-15$ seconds.

6. Stain in $0.1 \%$ safranin $\mathrm{O}$ solution for 5 minutes.

7. Dehydrate and clear with $95 \%$ ethanol, absolute ethanol, and xylene, using 2 changes each, 2 minutes each.

Results:

- GAGs: red

- Nuclei: black

- Cytoplasm: gray-green

- Cartilage, mucin, mast cell granules: orange-red 


\section{Alizarin Red protocol}

Fixation: Formalin fixed, OCT cryosectioned samples

Positive Control: bone

\section{Solution and Reagents:}

Alizarin Red Solution:

Alizarin Red S: 2 g

Distilled water: $100 \mathrm{ml}$

Mix well. Adjust the $\mathrm{pH}$ to $4.1 \sim 4.3$ with $10 \%$ ammonium hydroxide or hydrochloric acid. The $\mathrm{pH}$ is critical.

Acetone (100\%)

Acetone-Xylene (50/50)

Acetone $(100 \%): 50 \mathrm{ml}$

Xylene : $50 \mathrm{ml}$

\section{Procedure:}

1. Rehydrate samples on slides in distilled water for $5 \mathrm{~min}$.

2. Stain slides with the Alizarin Red Solution for 30 seconds to 5 minutes, observe the reaction microscopically.

3. Shake off excess dye and blot sections.

4. Dehydrate in acetone, 20 dips.

5. Then dehydrate in Acetone-Xylene (1:1) solution, 20 dips.

6. Clear in xylene and mount.

\section{Results:}

Calcium deposits (except oxalate) -------- orange-red

This precipitate is birefringent. 


\section{Immunohistochemical staining for Col I/II- protocol}

(Separate $2^{\circ}$ antibody and Fluoro labeling)

\section{NOTES:}

Reconstitute primary and secondary antibodies if lyophilized

Aliquot antibodies, proteinase $\mathrm{K}$ to prevent freeze/thaw cycles

Consumables:

Distilled water

Deionized, distilled water

PBS

PAP Pen

Permanent marker

Glass cover slides

Slide holder

Tupperware with lid

Aluminum foil

Pronase $(1 \mathrm{mg} / \mathrm{ml})$

Hyaluronidase

Proteinase K dilution: 20ug/ml (1:500 in PBS)

Blocking Buffer:

$10 \%$ goat serum, $1 \%$ bovine serum albumin in PBS

$1.25 \mathrm{ml}$ goat serum, $11.25 \mathrm{ml}$ PBS, $125 \mathrm{mg}$ BSA

\section{Primary incubation}

1:400 dilution in blocking buffer for collagen type I

1:5000 dilution in blocking buffer for collagen type II

$2 \mathrm{ml}$ blocking buffer and $5 \mathrm{ul}$ primary is good for $\sim 4$ sections

\section{Secondary incubation}

Biotinylated anti-mouse $\mathrm{f}(\mathrm{ab})$ fragment IgG antibody

1:50 dilution in blocking buffer

$2 \mathrm{ml}$ blocking buffer and 40ul secondary

Propidium iodide: stock solution made to $1 \mathrm{mg} / \mathrm{ml}$ in PBS

DILUTE stock solution to $1 \mathrm{ug} / \mathrm{ml}$ in PBS prior to staining

Label- AlexaFluor Streptavidin conjugated or Extravidin-FITC 1:50 dilution in PBS

2ml PBS and 40ul Label 


\section{Methods:}

Place slides in slide holder and rinse with distilled water

RT 5

Dry slides using Kimwipe, do not touch wipe to samples.

Surround samples with PAP pen and rinse samples in PBS

RT $2 \times 5$

Cover samples with Pronase $1 \mathrm{mg} / \mathrm{ml}$ in PBS

$37^{\circ} \mathrm{C} 30^{\prime}$

Rinse with PBS

RT $2 \times 5^{\prime}$

Cover samples with Hyaluronidase $1 \%$ in PBS

$37^{\circ} \mathrm{C} 30^{\prime}$

Rinse with PBS

Cover samples with proteinase $\mathrm{K}(20 \mathrm{ug} / \mathrm{ml})$

RT $2 \times 5^{\prime}$

RT 6'

Rinse with PBS

RT $2 \times 5^{\prime}$

Pre-incubate samples with blocking buffer

RT 2 hrs

Incubate samples in Primary Incubation

Overnight at $4^{\circ} \mathrm{C}$

Avoid sample dry-out: store slides in a covered Tupperware container with a damp $(\mathrm{dH} 2 \mathrm{O})$ cloth

Rinse with PBS

Incubate samples in Secondary Incubation

Rinse with deionized, distilled water

RT 3x5'

RT 1 hr

AVOID LIGHT! FOR STEPS 15 onward, work in dark!

RT 5

Incubate samples in propidium iodide $(1 \mathrm{ug} / \mathrm{ml})$

RT 15,

Rinse in deionized, distilled water

$3 \times 5$,

Cover samples with Label (extravidin or Alexa Fluor)

RT $1 \mathrm{hr}$

Mount with coverslip

Image immediately thereafter

Keep covered with aluminum foil at $4^{\circ} \mathrm{C}$ 


\section{RT-PCR protocol}

\section{EQUIPMENT:}

- Thermo cycle PCR machine

- Pen

- Appropriate holders

- Pipettors (10 and 100ul)

\section{CONSUMABLES:}

- Pipette tips (10-100ul)

- Kimwipes

- $1.5 \mathrm{ml}$ tubes

- PCR tubes

- Ice (crushed)

- Gloves

\section{CHEMICALS:}

- $70 \%$ isopropanol to sterilize

- RNA away to clean

- Random Primers

- Nuclease free $\mathrm{H}_{2} \mathrm{O}$

- dNTP (10 mM dNTP mix)

- 5x buffer

- $\quad 0.1 \mathrm{M}$ DTT

- RNAse out

- RNAse H

-enzyme keep in ice -enzyme keep in ice

- SuperScript II (SSII)

\section{Preparation}

1. Wear gloves all the time.

2. Clean the gloves and table surface with isopropanol and RNA away.

3. Put chemical needed to make mix 1 and 2 in the crushed ice (except enzymes).

4. Label tubes (samples and mix tubes).

- If there are strip tubes that can no longer be used for qPCR because they are not the right type of tube, these can be used for RT reactions.

5. Calculate amount of water and RNA to pipette for each sample:

- $\quad$ NA $=300$ ng of RNA

- $\mathrm{X}=$ volume of RNA that is equal to 300ng

○ $\mathrm{Y}=$ volume of water that, when added to $\mathrm{X}$, equals $10.66 \mu \mathrm{L}$ volume

- Superscript II can be used with up to 500ng of mRNA. We have chosen to use 300ng/rxn for RT experiments.

- Random Primers can be 50-250ng/reaction. We have chosen to use 100ng/rxn for RT experiments. 
6. Switch PCR machine on and load appropriate program (details in the PCR machine short guide).

7. Calculate amount of each chemical needed for number of run reactions according to procedure below.

NOTE: Calculating we assume that we will loose some of the mix for pipetting error so always multiply by number of reactions $+10 \%$

Example:

If you planning to run 10 reactions. Calculate amount of each chemicals for 11 reactions.

One RT-reaction is typically enough RNA to run a single plate with six different genes. Therefore, it is beneficial to perform several RT reactions with the same RNA at any given time. Additionally, cDNA is more stable than RNA, and therefore will be less likely to degrade over time at $-20^{\circ} \mathrm{C}$.

Reaction Conditions:

- 300 ng of RNA

- 100 ng of Random Primers

- $0.25 \mu \mathrm{l}$ (50 units) SS II

Mix 1:

dNTP

$\mathbf{1} \boldsymbol{\mu l}$

Random Primers $\quad 0.33 \boldsymbol{\mu l}$ (from diluted tube- have to be $100 \mathrm{ng}$ )

RNA X-depend on RNA concentration (have to be $300 \mathrm{ng}$ )

$\mathrm{H}_{2} \mathrm{O} \quad Y$ - to complete $12 \mu \mathrm{l}$

TOTAL: $\quad \mathbf{1 2} \boldsymbol{\mu l}$ per PCR tube

$R N A$ and $H 2 O$ are pipetted into PCR tubes individually; not mixed in Mix 1

\section{Mix 2:}

5x buffer $\quad 4 \mu \mathrm{l}$

$0.1 \mathrm{M}$ DTT $\quad 2 \mu \mathrm{l}$

RNAse out $\quad 1 \mu \mathrm{l}$ (enzyme add at the end, keep in ice) -destroy RNAse

TOTAL: $\quad 7 \mu$ per PCR tube

\section{SS II:}

TOTAL: $1 \mu \mathbf{l}$ (if 200 units than $0.25 \mu \mathrm{SSII}+0.75 \mu \mathrm{l} \mathrm{H}_{2} \mathrm{O}$ ) -enzyme keep in ice

RT Reaction TOTAL: $\mathbf{2 0} \boldsymbol{\mu l}$




\section{Procedure}

1. Pipette calculated amount of dNTP and Random Primers to mix 1 tube.

2. Take RNA samples from $-80^{\circ} \mathrm{C}$ freezer and place them in ice to thaw.

3. Pipette $\mathrm{H}_{2} \underline{\mathrm{O}}$ to each PCR tube (since tubes are empty you do not have to change pipette tips every time).

4. Pipette RNA to each PCR tube which contains water already. Mix gently with pipettor. Change pipette tips every time.

5. Put RNA samples back to $-80^{\circ} \mathrm{C}$ freezer.

6. Pipette 1.33 ul to each tube from mix 1 tube. Mix gently with pipettor. Change pipette tips every time.

7. Close tubes tightly and place them in the PCR machine, start program (how to start the program look at the short PCR machine guide).

8. While waiting prepare mix 2 by pipetting calculated amount $\underline{5 x \text { buffer and } 0.1 \mathrm{M}}$ DTT. Do not pipette RNAse out till the last moment. Mix gently with pipettor.

9. Pause PCR machine (how to pause PCR machine look at short guide), take the samples out and chill them in ice, while waiting pipette calculated amount of RNAse out to mix 2 tube. Mix gently with pipettor.

10. Pipette 7 ul of mix 2 to each tube. Mix gently with pipettor. Change pipette tips every time.

11. Close tubes tightly and place them back to PCR machine, restart the program (how to restart the program look at short PCR machine guide).

12. While waiting prepare SuperScript II (SSII) mix. Pipette SSII in the last moment. Mix gently with pipettor.

13. Pause PCR machine, take the samples out and add lul of SSII mix to each tube. Mix gently with pipettor. Change pipette tips every time.

14. Close tubes tightly and place them back to PCR machine, restart the program.

15. If run

\section{a. With break}

i. Stop PCR machine.

ii. Load second part of PCR program.

iii. Pipette to each tube $0.4 \mathrm{ul}$ of the RNAse H-keep the enzyme cold. Mix gently with pipettor. Change pipette tips every time.

\section{b. Without break}

i. Pause PCR machine (how to pause the program look at short PCR machine guide).

ii. Take tubes out from PCR machine and pipette $0.4 \mathrm{ul}$ of the RNAse $\mathrm{H}$ to each tube. Mix gently with pipettor. Change pipette tube every time.

iii. Close tubes tightly and place them in the PCR machine, restart program (how to restart the program look at short PCR machine guide).

16. When program is finished, take tubes out from PCR machine and place them in $20^{\circ} \mathrm{C}$ freezer for long storage.

17. Switch off PCR machine. 
Find RT program on thermocycler machine (make sure protocol is similar to below) or:

Reprogram thermocycler using the following protocol

Protocol

1. $65^{\circ} \mathrm{C}$

$5 \min -\operatorname{mix} 1$

chill on ice \& add mix 2

2. $25^{\circ} \mathrm{C}$

$2 \min$

add SS II \& mix well (enzyme keep cold)

3. $25^{\circ} \mathrm{C}$

4. $42^{\circ} \mathrm{C}$

$10 \mathrm{~min}$

5. $70^{\circ} \mathrm{C}$

$50 \mathrm{~min}$

$15 \mathrm{~min}$

break if needed

a. $4^{\circ} \mathrm{C} \quad 5 \mathrm{~min}$

b. $10^{\circ} \mathrm{C}$ forever

Add $0.4 \mu \mathrm{l} \underline{\text { RNAse H }}$ (enzyme keep cold) -destroy RNA

6. $37^{\circ} \mathrm{C} \quad 20 \mathrm{~min}$

7. $65^{\circ} \mathrm{C} \quad 20 \mathrm{~min}$

8. $4^{\circ} \mathrm{C} \quad 5 \mathrm{~min}$

9. $10^{\circ} \mathrm{C} \quad$ forever

TOTAL: $127 \mathrm{~min}$ (without break) 


\section{Real Time PCR (QPCR) protocol}

Time of set up: $90 \mathrm{~min}$ ( $\sim 30 \mathrm{~min}$ work in the dark)

Time of reaction: $2.5 \mathrm{~h}$

Total time: $4 \mathrm{~h}$

\section{Supplies}

- Tube stripes or plates with covers

- Pipette with filter pipette tips

- RNA/DNA free tubes for master mix dilution

- RNA/DNA free tubes for cDNA and Primers mixes

- Appropriate Holders

- Dark room with the hood

- qPCR machine in room 154 of Forestry building

\section{Chemicals:}

- Nuclease free water (in freezer)

- SYBR (light and temperature sensitive, aliquot after arrival and minimize number of refreeze, keep in the aluminum foil, in freezer)

- Primers Forward (FWD) and Reverse (REV) (in freezer)

- Sample cDNA (in freezer)

- RNAase away in spray bottle

- 70\% isopropanol in spray bottle

- Crushed ice

\section{Chemical Recipes}

cDNA mix:

$0.5 \mathrm{uL}$ cDNA (15 ng)

$1.5 \mathrm{uL}$ Nuclease free $\mathrm{H}_{2} \mathrm{O}$

Total: $2.0 \mathrm{uL}$ to each well

Primer mix:

$0.5 \mathrm{uL}$ Fwd primer

$0.5 \mathrm{uL}$ Rev primer

$1.0 \mathrm{uL}$ Nuclease free $\mathrm{H}_{2} \mathrm{O}$

Total: $2.0 \mathrm{uL}$ to each well

Master mix:

$12.5 \mathrm{uL} \mathrm{SYBR}$

$7.5 \mathrm{uL}$ Nuclease free $\mathrm{H}_{2} \mathrm{O}$

Total: 20.0 uL to each well

Note: Each sample will be run in duplicates. 


\section{Procedure}

Pre-preparation:

1. Wipe down pipettes first with isopropanol and then with RNAase Away.

2. Fill bowls with crushed ice.

3. Remove all chemicals needed from freezer.

4. After melted, centrifuge each chemical (except the Sybr-Green).

5. Put all chemicals on ice. Keep SYBR-Green covered with aluminum foil until needed.

6. Wipe down the hood with isopropanol.

7. Turn the blower and the light on in the hood.

8. Conduct all procedures from now on in the hood.

9. Spray gloved-hands with isopropanol and RNAase Away. If anything outside of the hood is touched, spray gloved-hands again.

Hints: It is helpful to make all the recipes for the mixes (formulas down below) and tube strip grid beforehand. Mix all chemicals by pipetting or flicking before transferring. Be careful mixing Sybr-Green, it is very sensitive to degradation.

\section{Standard curve:}

The standard curve procedure is not included in this protocol. The standard curve only needs to be completed when the genes of interest have never been tested for in qPCR. Otherwise, use old efficiency values for the specific gene.

Preparation of the cDNA mix (need Calibrator and NTC along with sample cDNA):

1. You will prepare a separate cDNA mix for each cDNA being tested, but all calculations will be the same for each cDNA mix.

2. Calculate how much of the cDNA mix components you will need (in $\mathrm{uL}$ ):

a. $\quad \mathrm{cDNA}=(\#$ of wells specific cDNA will be in +1$) * 0.5$

b. Nuclease free $\mathrm{H}_{2} \mathrm{O}=$ (of wells specific cDNA will be in +1$) * 1.5$

3. Get out $\mathrm{X}$ RNA/DNA free tubes. ( $\mathrm{X}=$ the number of $\mathrm{cDNAs}$ being tested)

4. Label the RNA/DNA free tubes with a symbol of the cDNA which will be run.

5. Fill each tube with the calculated (in \# 2) amount of nuclease free water. You do not have to change the pipette tips each time.

6. Fill each tube with the calculated (in \# 2) amount of cDNA (specific for each cDNA). Change the pipette tip every time. Mix each gently with the pipette. The cDNA for the NTC is Nuclease free $\mathrm{H}_{2} \mathrm{O}$.

Preparation of the Primer mix:

1. You will prepare a separate primer mix for each gene being tested, but all calculations will be the same for each primer mix.

2. Calculate how much of the primer mix components you will need (in $\mathrm{uL}$ ):

a. $\quad$ F Primer $=(\#$ of wells specific primer will be in +2$) * 0.5$

b. $\quad$ R Primer $=(\#$ of wells specific primer will be in +2$) * 0.5$ 
c. Nuclease free $\mathrm{H}_{2} \mathrm{O}=((\#$ of wells specific primer will be in +2$) * 1.0)$

3. Get out X RNA/DNA free tubes. ( $\mathrm{X}=$ the number of genes being tested)

4. Label the RNA/DNA free tubes with a symbol of the gene which will be run.

5. Fill each tube with the calculated (in \# 2) amount of nuclease free water. You do not have to change the pipette tips each time.

6. Fill each tube with the calculated (in \# 2) amount of Forward and then Reverse primers (specific for each gene). Change the pipette tip every time. Mix gently with the pipette.

Preparation of master mix:

1. Calculate how much of the master mix components you will need (in ul):

a. SYBR Green $=(\#$ vertical well depth* \# horizontal well depth +5$) * 12.5$

b. Nuclease free $\mathrm{H}_{2} \mathrm{O}=((\#$ vertical well depth* \# horizontal well depth +5$)$ $* 7.5$

2. Label the tube for master mix and fill it with the calculated (in number 1) amount of nuclease free water.

3. Turn off the lights.

4. In the dark, pipette the calculated (in \# 1) amount of SYBR to the master mix tube. Mix gently with the pipette.

5. Cover with aluminum foil.

QPCR tube strips (or plates) creation (you can reverse axis if desired):

\begin{tabular}{|c|c|c|c|c|c|c|c|c|c|c|c|c|}
\hline cDNA & Genes $\rightarrow$ & & & & & & & & & & & \\
\hline$\downarrow$ & 18s rRNA & Gene 1 & Gene 3 & Gene 4 & Gene 5 & Gene 6 & Gene 7 & Gene 8 & Gene 9 & Gene 10 & Gene 11 & Gene 12 \\
\hline Calibrator & & & & & & & & & & & & \\
\hline Calibrator & & & & & & & & & & & & \\
\hline NTC & & & & & & & & & & & & \\
\hline NTC & & & & & & & & & & & & \\
\hline cDNA 1 & & & & & & & & & & & & \\
\hline cDNA 1 & & & & & & & & & & & & \\
\hline cDNA 2 & & & & & & & & & & & & \\
\hline CDNA 2 & & & & & & & & & & & & \\
\hline
\end{tabular}

or

\begin{tabular}{|c|c|c|c|c|c|c|c|c|c|c|c|c|}
\hline cDNA & Genes $\rightarrow$ & & & & & & & & & & & \\
\hline$\downarrow$ & $18 s, R N A$ & $18 s$ rRNA & Gene 1 & Gene 1 & Gene 2 & Gene 2 & Gene 3 & Gene 3 & Gene 4 & Gene 4 & Gene 5 & Gene 5 \\
\hline Calibrator & & & & & & & & & & & & \\
\hline NTC & & & & & & & & & & & & \\
\hline cDNA 1 & & & & & & & & & & & & \\
\hline cDNA 2 & & & & & & & & & & & & \\
\hline cDNA 3 & & & & & & & & & & & & \\
\hline cDNA 4 & & & & & & & & & & & & \\
\hline cDNA 5 & & & & & & & & & & & & \\
\hline cDNA 6 & & & & & & & & & & & & \\
\hline
\end{tabular}

1. Pipette $2 \mathrm{uL}$ of specific primer mix to each well. Each specific primer mix will go vertical. The pipette tip only needs to be changed between genes.

2. Pipette $2 \mathrm{uL}$ of specific cDNA mix to each well. Each specific cDNA mix will go horizontal. The pipette tip needs to be changed each time. 
3. In the dark, pipette $21 \mathrm{uL}$ of master mix to each well. The pipette tip needs to be changed every time.

4. In the dark, put the cover tightly on the tubes and cover with aluminum foil.

5. Carry the tubes to the Forestry building.

6. In room 147 and in the dark, mini centrifuge each tube strip. Re-cover with aluminum foil.

7. Carry the tubes to the real-time machine (room 154 in the Forestry building).

8. Set up the input file (can be prepared earlier). When creating a new file, click on the calibration option. To insert of dissociation curve, import it from an existing file.

Plate Set up

1. Mark wells as:

Unknown $=$ samples

$\mathrm{NTC}=$ for nonspecific control

Calibrator $=$ calibrator

2. For all wells in use, choose the universal mix as Sybr Green (SYBR)

3. Mark duplicates.

4. Give names to all wells by going to the grid view and typing the name in.

Thermal Profile:

Hot start

$95^{\circ} \mathrm{C} / 15 \mathrm{~min}$

Beginning of the cycle

$95^{\circ} \mathrm{C} / 15 \mathrm{sec}$

$\mathrm{X}^{\circ} \mathrm{C} / \mathrm{Z}$ sec $\leftarrow$ determined empirically for specific primers

$72^{\circ} \mathrm{C} / 40 \mathrm{sec}$

End of the cycle

Run for 40 cycles

Dissociation cycle

9. In the dark, place each stripe in the assigned column in the real time machine.

10. Let the machine lamp warm up for 20 minutes. Choose the option that the test will begin after lamp is warmed up.

11. Check the box that will switch off the lamp after the run is completed

12. Run the open file where the conditions of the experiment are specified (each run takes around 2.5 hour).

\section{$\underline{\text { Results analysis }}$}

Data analysis is carried out using Pfaffl's method according to the equation:

ratio $=\frac{\left(E_{t \arg e t}\right)^{\Delta C_{t} t \arg e t(\text { control-treated })}}{\left(E_{\text {ref }}\right)^{\Delta C_{t} r e f(\text { control-treated })}}$ 
Where: $C_{t^{-}}$cycle number in the linear range of amplification, $E$ - efficiency of the process for each gene, target- gene of interest, ref- housekeeping gene, control- calibrator, treated-sample

NOTE: Remember where you read Ct values form QPCR software set your threshold line constant for each gene through whole experiment. 


\section{Western blotting protocol}

\section{Protein Isolation:}

1. Using ice-cold RIPA buffer ( $3 \mathrm{~mL} / 1 \mathrm{~g}$ tissue), lyse tissue and homogenize.

2. Centrifuge at $14000 \mathrm{~g}$ for 10 minutes at $4^{\circ} \mathrm{C}$.

\section{Preparing Total Protein Sample for Western Blot}

1. After obtaining concentration of total protein in sample using Lowry method, calculate necessary amount of sample solution that will give $\geq 10$ ug of total protein, and place in a microcentrifuge tube. Do this for each sample because the solution amounts will most likely be different for each.

2. Combine sample and deionized water to $54.5 \mathrm{ul}$ volume

3. Buffer used is $4 \mathrm{X}$ LDS PAGEgel buffer (25ul)

4. Add $10 \%$ Beta-mercaptoethanol in hood with fan on! (20.5ul)

5. Perform the above steps for marker and std. protein, using 10ul of marker/protein and $44.5 \mathrm{ul} \mathrm{ddH} 2 \mathrm{O}$

6. Heat mixture for 10 minutes at $70^{\circ} \mathrm{C}$ in hot water bath

7. Flick and centrifuge sample $\leq 10^{\prime}$ to mix solution, and bring sample to bottom of tube

\section{Running a Gel:}

1. Remove ready-gel from storage pouch. The comb has been removed from the gel already.

2. Open cams to release existing casting plate

3. Place gel cassette sandwich into the slots at the bottom of each side of the electrode assembly. Be sure the short plate of the gel cassette sandwich faces inward toward the notches of the U-Shaped gasket

4. Life the gel cassette sandwich into place against green gasket and slide into clamping frame

5. Press down on the electrode assembly while closing the two cam levers of the clamping frame to format the inner chamber and to insure a proper seal of the short plate against the notch of the U-Shaped gasket. Short plate must align with notch in gasket

6. Lower the inner chamber assembly into the minitank. Fill the inner chamber with $200 \mathrm{~mL}$ running buffer until the level reaches halfway between the top of the taller and shorter glass plates of the gel cassettes. Check for leaks. Do not overfill, may cause problem with transfer.

7. Add $600 \mathrm{~mL}$ running buffer to minitank, or lower chamber.

a. PAGEgel Running buffer MUST be used!

b. Make $600 \mathrm{~mL}$ of 1xRunning buffer (dilute 30mL 20X PAGEgel Running Buffer with $570 \mathrm{~mL}$ ultrapure water for a run).

c. The buffer may be reused on the outer (anode) side, but fresh buffer is always required on the inner (cathode) chamber 
d. When reusing buffer, dilute $10 \mathrm{~mL}$ of PAGEgel Running Buffer (20X) to $200 \mathrm{~mL}$ with ultrapure water for the inner chamber

Gel Loading

1. Spin down solution before beginning to get all liquid to the bottom of the tube

2. Be sure all bubbles are out of the wells before beginning. Do this by tapping on the outside walls of the gel to get them out.

3. Always use small pipette tips. Large ones separate gel walls decreasing the volume to level below wells make it very difficult to load the right amount

4. When obtaining sample, wipe pipette tip on side of microcentrifuge tube to remove excess

5. Rinse wells with ddH2O twice, then fill with $\mathrm{ddH} 2 \mathrm{O}$ before loading.

6. To load gels, guide the pipette with other hand index finger near the tip of the pipette and press tip against the back of the front surface of the gel and guide it into the well. Release all fluid without over-pushing on the pipette.

Running:

1. Attach to power supply.

2. Place cover on cell in color coded fashion.

3. Attach leads in color coded fashion to power supply

4. Set voltage requirement to $175 \mathrm{VDC}$ and all the amps to vary starting at $80 \mathrm{~mA} / \mathrm{gel}$

5. When reusing running buffer in the anode side (outer chamber), run at $150 \mathrm{VDC}$, starting at $60 \mathrm{~mA} / \mathrm{gel}$.

6. Depress the run button on the power supply. Should see effervescence due to hydrolyses of solution caused by electrical current

7. Gel should be run to bottom within 35-90'. Need to watch.

\section{PROTEIN TRANSFER}

1. Make $1 \mathrm{~L}$ of transfer buffer made with $10 \%$ methanol (dilute to $20 \mathrm{x}$ instead of 10x)

a. Transfer buffer: $50 \mathrm{~mL}$, Methanol: $100 \mathrm{~mL}$, ddH2O: $1850 \mathrm{~mL}$

2. Cut membrane and filter paper to dimensions of gel (Wear gloves when handling membranes)

3. Equilibrate gel and soak filter paper and fiber pads in transfer buffer for $15 \mathrm{mins}$

a. Removes contaminating electrophoresis buffer salts.

4. PVDF membranes must first be wetted in $100 \% \mathrm{MeOH}$ and then soaked in transfer buffer for 15 minutes. Do this while running gels.

5. Prepare gel sandwich order:

a. Gray side of cartridge down

b. Fiber pad 


\section{c. Filter paper}

6. Gel-remove ridge and excess well strippings and cut one corner for location purposes using a piece of plastic or other sharp edge. Place face up on filter paper.

a. Membrane

b. Filter paper

c. Fiber pad

d. Close it up

7. MAKE SURE there are no bubbles present anywhere at any time during creation of sandwich! Roll them out with a glass rod or put on glove and use fingertips.

8. Membrane must be on positive side of transfer case in order to allow the negatively charged proteins to relocate to the membrane from the gel.

9. Place cassette in module

10. Add cooling unit with ice shavings to opposite end. If ice shavings not available, fill cooling unit with water and place in freezer until frozen.

11. Fill with transfer buffer. Transfer cell capacity, $650 \mathrm{~mL}$ with cooling unit inserted.

12. Put on lid and connect to power supply in color coded fashion

a. 50Volts, 2-4hrs, expected current around $250 \mathrm{~mA}$

13. Upon completion, disassemble sandwich and remove membrane for development

14. Clean cell, fiber pads, and cassettes with lab detergent and rinse well with deionized water

15. Filter pads and gel can be pitched if transfer was successful

\section{MEMBRANE BLOCKING}

1. Wet the membrane in PBS for several minutes. If using a PVDF membrane that has been allowed to dry, pre-wet briefly in 100\% methanol and rinse with double distilled water before incubating in PBS.

2. Block the membrane in BSA blocking buffer for 1hour. Be sure to use sufficient blocking buffer to cover the membrane (a minimum of $0.4 \mathrm{~mL} / \mathrm{cm}^{2}$ is suggested)

3. Membranes can be blocked overnight at $4{ }^{\circ} \mathrm{C}$ if desired

4. DO NOT add TWEEN-20 when blocking the membrane. The membrane should not be exposed to Tween-20 until blocking is completed

5. DO NOT USE CASEIN with biotynylated antibodies

6. Dilute the primary antibody in BSA blocking buffer. Optimum dilution depends on antibody and should be determined empirically. A suggested starting range is 1:1000 or 1:5000. To lower background, add $0.1-0.2 \%$ Tween-20 to diluted antibody before incubation.

7. Biotinylated Anti-porcine IL-1 $\alpha$ Antibody can be used at $0.1-0.2 \mathrm{ng} / \mathrm{mL}$

8. Incubate blot in $1^{\circ}$ for 60 " or longer at room temp with gentle shaking. Use enough antibody solution to completely cover membrane.

9. Was membrane $4 \mathrm{x}$ for 5 " each at room temp in PBS $+0.5 \%$ Tween-20 wth gentle shaking using a generous amount of buffer.

10. Dilute the fluorescently-labeled $2^{\circ}$ antibody in BSA blocking buffer. Avoid prolonged exposure of antibody vial to light. Suggested dilution in 1:5000 to 
1:20,000 with 1:10,000 as a good starting point. Add Tween-20 to the diluted antibody as you did for the $1^{\circ}$ antibody. Add SDS if desired.

11. For detection of small amounts of protein, try using more $2^{\circ}$ antibody

12. Be careful not to introduce contamination into antibody vial

13. Diluted $2^{\circ}$ antibody can be saved and reused. Store at $4^{\circ} \mathrm{C}$ and protect from light. However, for best sensitivity and performance, use freshly diluted solution

a. Adding $0.01 \%-0.02 \%$ SDS to the diluted $2^{\circ}$ antibody in addition to Tween-20 will substantially reduce membrane background, particularly when using PVDF. However DO NOT use SDS during blocking or to the diluted $1^{\circ}$ antibody.

14. Incubate blot in $2^{\circ}$ antibody for $30-60 "$ at room temp with gently shaking. Protect from light during incubation

15. Allowing incubation to proceed more than 60 " may increase background

16. Wash membrane $4 \mathrm{x}$ for 5 " each at room temp in PBS $+0.1 \%$ Tween-20 with gentle shaking. Protect from light.

17. Rinse membrane with PBS to remove residual Tween-20. The membrane is now ready to scan.

18. Scan in appropriate channels using the LICOR Odyssey

19. Protect membrane from light until it has been scanned

20. Keep membrane wet if you plan to strip and reuse it. Once membrane has dried, stripping is ineffective

21. Blots can be allowed to dry before scanning if desired. Signal strength may be enhanced on a dry membrane. The membrane can also be rewetted for scanning

22. The fluorescent signal on the membrane will remain stable for several months, or longer, if protected from light. Membranes may be stored dry or in PBS buffer at $4^{\circ} \mathrm{C}$

23. If signal on membrane is too strong or too weak, re-scan membrane at lower or higher scan intensity setting, respectively.

Running Buffer for use with PAGEgels:

Standard SDS Running Buffer, 20x for Reduced Samples (CB60500)

( $0.8 M$ Tricine, $1.2 M$ Tris, $2 \%$ SDS, $50 \mathrm{~m} M$ Sodium Bisulfite)

Tricine (free acid)* $71.7 \mathrm{~g}$

Tris (free base)* $\quad 72.6 \mathrm{~g}$

SDS $\quad 10.0 \mathrm{~g}$

Sodium Bisulfite $\quad 2.5 \mathrm{~g}$

Ultra-pure water to $500 \mathrm{ml}$

$\mathrm{pH}$ should be between 8.2 and 8.3 at $25^{\circ} \mathrm{C}$.

For non-reduced samples (especially antibodies), omit the Sodium Bisulfite. For DNA and Native PAGE, omit both the bisulfite and the SDS; pH is slightly higher. 
Transfer Buffer for use with PAGEgels:

Tris-Glycine-SDS Transfer Buffer, 10x (CB82500)

(0.25M Tris, $1.92 M$ Glycine, $0.1 \%$ SDS $)$

Tris (free base)* $\quad 15.2 \mathrm{~g}$

Glycine* $\quad 72.1 \mathrm{~g}$

SDS $\quad 5.0 \mathrm{~g}$

Ultra-pure water to $500 \mathrm{ml}$

$\mathrm{pH}$ should be between 8.5 and 8.6 at $25^{\circ} \mathrm{C}$. 


\title{
Drop sled control protocol
}

\author{
Required components \\ - National Instruments DAQ card \\ - NI USB 6008 \\ - Relay in enclosure \\ - Rails/stand \\ - $1.75 \mathrm{~kg}$ drop sled \\ - $\mathrm{PCB}$ accelerometer and specialty platen \\ - Hexcell \\ - Solenoids \\ - Rabbit "bed" \\ - Foot "boot" \\ - Velcro (2sided) \\ Directions
}

1. Return drop sled to bottom of rails

2. Apply graphite lubricant to rails

3. Plug in NI $6008 \mathrm{~A} / \mathrm{D}$ USB to USB port on computer with LabView program

4. Plug in co-axial cable into relay box

5. Plug relay into outelet

a. Solenoids are in parallel

6. Open Labview

a. Open Dropsled.vi

b. Run program

7. Plug in force transducer to DAQ card using microdot cable
a. DO NOT BEND OR CRIMP microdot cable!

8. Use toupee tape and/or duct tape to adhere Hexcell to load cell platen

9. Test DAQ

a. Make sure indentation for knee on Hexcell is aligned appropriately

a. Green light on

10. Balance load cell

i. Allow to run for a few minutes

11. Turn green light of

12. Align knee using Velcro straps

a. Impactor head (Hexcell) should be *just* proximal to patella

b. Knee should be flexed to slightly less than 90 degrees

c. Make sure impaction will load tibia axially. If off, it will lead to gross bone fracture

13. Use manual control of solenoids to move impactor up and down

14. Once alignment is properly ensured, raise impactor to appropriate height

15. Set .vi program seting to automatic

a. Pick appropriate threshold

16. Impact knee by pressing "Release" button when ready 


\section{Rabbit handling and euthanasia protocol}

Materials

Acepromazine: from FisherSci; $10 \mathrm{mg} / \mathrm{ml}$ Vedco

Potassium chloride: $2 \mathrm{mmol} / \mathrm{kg}$ [100g KCl dissolved into $1000 \mathrm{~mL}$ ddH2O)

$10 \mathrm{cc}$ hypodermic needles, 21 gauge needles or smaller

$5 \mathrm{cc}$ hypodermic needles, 21 gauge needles or smaller

JD Medical VT-110 small animal anesthesia unit: includes, vaporizer, (0.5-1L) Bain's non-rebreather bag, oxygen flowmeter, oxygen regulator, high pressure hose for oxygen, anesthesia filter, sodasorb for rebreather if needed, proper adapters for hoses, nose cone (large canine)

Handling

1. Grab animal behind head on scruff of neck

2. Handle by skin and support weight of animal with hand under stomach or around rear.

3. Keep animal's face tucked in your elbow

Inspect all equipment before performing euthanasia. This includes making sure that the rubber on the nose cone and non-rebreathing bag do not have holes or deformities. Make sure oxygen tank is full and isofluorane is full.

Euthanasia

1. Weigh animal

2. Use scale with containment

3. Tranquilize with acepromazine (maleate injection)

a. Use $0.5 \mathrm{mg} / \mathrm{lb}$

4. Err on higher dosage of tranquilizer. For example, if the animal weighs $12 \mathrm{lbs}$, tranquilizer dosage will be $0.6 \mathrm{ml}$. Give animal $>0.6 \mathrm{~mL}$.

5. Inject the tranquilizer under the animal's fur. Pull up on nape of neck, inject into the fold of skin where there is a "void" between skin and musculature. Skin will make an "A" shape.

6. RELAX: Return animal to cage to distress and all tranquilizer to set in

7. Make sure Isofluorane levels are high enough in tank of vaoporizer.

8. Turn on oxygen to $1 \%$ (black ball should rise to $1 \%$ )

9. Euthanize: Turn on isofluorane to $5 \%$ using knob

10. One person should hold the animal from the side, making sure its face is easy to get to with the mask

11. Another person should hold the nose cone to the animal's face

12. Non-rebreather bag should be closed

13. Cover animal's mouth and nose completely with nose cone

a. It is ok if the animal pushes their face into the nose cone; However, avoid discomfort of the animal by preventing the rubber of the cone to touch their eyes

14. Animal should be restrained by one person throughout this process in order to prevent escape during delirium 
15. Hold scruff to prevent their nose and mouth from coming out of the nose cone

16. Use extreme caution not to breathe vapors or gas

17. Watch animal's breathing and prepare both nose cone holder and rabbit restrainer for delirium

18. Avoid animal's nose from getting pushed against inside of nose cone

19. After fight/flight delirium, the animal will become limp. Maintain gas flow but reduce to $1-3 \%$ isofluorane flow.

20. Watch and listen for heart beat. Overdoes of isofluorane will euthanize the animal.

21. If the animal does not become successfully euthanized after $\sim 5 \mathrm{~min}$ post-delirium, use intracardial injection of potassium chloride

22. Intracardial injection

a. Should only be performed by a trained professional! 


\section{Acute response - Rabbit dissection protocol}

Supply Check List:

- $70 \%$ isopropanol

- RNALater

- Sterile 24-well trays

- Dulbecco's Modified Eagles Medium (DMEM)

- Ham's F12

- Fetal Bovine Serum (FBS)

- Penecillin/Streptomyicin $(\mathrm{P} / \mathrm{S})$

- Sterile Dissection tools - scalpel blades, tweezers, scissors, etc...

1. Make Growth and Flow Media

a. Media: $48.5 \%$ DMEM/F-12, 2\% FBS, 1\% Penn/Strep

a. Place all media ingredients into $37^{\circ} \mathrm{C}$ water bath for $\sim 15 \mathrm{~min}$.

i. Mix media in culture hood:

ii. $40 \mathrm{ml}$ media per animal

b. Make sure media is $37^{\circ} \mathrm{C}$ before use with any tissue.

2. Dissection and Explant Removal

a. Dissect limb from hip joint. Spray fur with isopropanol to wet hair and remove skin. Spray with isopropanol but be careful not to get any under the musculature.

b. Dissect fresh rabbit knee in culture hood using aseptic technique and sterile tools only.

c. Image tibial plateaus (with intact menisci) and femoral condyles using a digital camera.

i. Undercut facets leaving 3-4 $\mathrm{mm}$ of bone with rotary tool, irrigate with sterile PBS continuously.

ii. Rinse twice in sterile PBS, then rinse three times in culture media.

iii. Place tissue pieces in individual wells of a 6-well plate, keeping top surface up.

iv. Fill wells with $\sim 1 \mathrm{ml}$ of growth media. Make sure to cover all tissue with growth media. Place lid on wells.

d. Incubate for $24 \mathrm{hr}$ in $37 \mathrm{C}$ with $95 \%$ humidity to help maximize the percentage of dead cells

i. Place in incubator for 12 hours

ii. After 12 hours of incubation, remove media and store at $-80^{\circ} \mathrm{C}$. Replace immediately with fresh, $37^{\circ} \mathrm{C}$ growth media. Place in incubator for 12 more hours.

iii. After $24 \mathrm{hrs}$ total incubation time, remove media with pipette, store at $-80^{\circ} \mathrm{C}$

e. For cell viability of meniscal sections: 
i. Remove medial and lateral meniscus and place in dish containing sterile Phosphate Buffered Saline 1X (PBS)

ii. Place meniscus on clean surface (Plexiglass)

iii. Mark bottom of meniscus with India Ink

iv. Slice coronal sections from central-substance of both medial and lateral meniscus for 100-200um sections using custom slicing device. Include tears in slice if present.

v. Place remaining anterior and posterior tissue of menisci in RNAlater for separate study.

vi. Proceed to cell viability protocol.

f. For cell viability of cartilage sections:

i. Adhere cut surfaces of bone to plexiglass using cyanoacrylate. Allow $\sim 2$ min to fully cure.

ii. Use diamond saw to cut coronal sections of tibial plateau from the posterior region (posterior of the cruciate ligaments). Irrigate sections continuously during cutting with saline or PBS.

iii. Obtain 2-3 full-thickness slices of cartilage/subchondral bone for cell viability.

iv. Proceed to cell viability protocol as directed by manufacturer. 


\section{Cell viability protocol}

- $\quad$ LIVE/DEAD cytotoxicity kit (Invitrogen)

- ddH2O

- PAP pen

- Incubation trays or petri dishes, covered in foil

1. Remove reagents from freezer and allow to thaw to room temperature.

2. Place thin slices of tissue in petri dish and circle with PAP pen. Keep sample hydrated with sterile PBS.

3. Add $20 \mathrm{ul}$ of supplied $2 \mathrm{mM}$ EthD-1 stock solution to $10 \mathrm{~mL}$ sterile PBS. Vortex to ensure thorough mixing.

4. Combine reagents by transferring $5 \mathrm{uL} 4 \mathrm{mM}$ calcein $\mathrm{AM}$ stock solution to $10 \mathrm{~mL}$ EthD-1 solution.

5. Resulting solution is $2 \mathrm{uM}$ calcein $\mathrm{AM}$ and $4 \mathrm{uM}$ EthD-1 working solution.

6. Add 100-200uL of working solution to sample, be sure to cover the entire sample with solution. Cover the dish to prevent the samples from drying out and incubate samples for 30-45min at room temperature.

7. At the end of incubation, rinse samples with PBS. Mount onto slides using finetipped forceps or cover with plastic cover slips in petri dish.

8. Image using fluorescence microscopy. 
Appendix C: Copyright 


\section{Copyright permission (Figure 1-1 \& 1-6)}

From: mlkillia@mtu.edu

To: ddc@mtu.edu

Attachments: Nucleus Copyright.jpg (14.1KB)

Hi, Deb!

Will this work for copyright privileges in my dissertation? Should I just print out the

email and add it in the appendices (and add the Copyright line beneath the images)?

Thank.s-

Megan

Megan L. Killian, M.S.

Doctoral Candidate- Biomedical Engineering

Michigan Technological University

902 R.L. Smith Building

Mechanical Engineering-Engineering Mechanics

Houghton, MI 49931

mlkillialmtu.edu

Follow me on Twitter! : megankillian

----- Forwarded Message -----

From: "Niky Scragg" <nscragg@nucleusinc.com>

To: "Megan L. Killian" <mlkilliaemtu. edu>

Sent: Friday, September 24, 2010 2:25:52 PM GMT -05:00 US/Canada Eastern

Subject: Re: Medical Illustrations for Your Project

Dear Megan,

Thank you for your recomendation of the SMART Imagebase. The

requested images and Nucleus copyright line are attached. Please let

me know that you have received everything you need.

Kind regards,

Niky Scragg

Content Licensing coordinator

nscraggenucleusinc.com

http://Www. nucleusine.com

Toll free: 800.333 .0753

Phone: 770.953 .6566

Fax: 770.805 .0430

Nucleus Medical Media, Inc.

1275 Shiloh Road

Suite 3130

Kennesaw, GA 30144

[image/jpeg:DR00038_40018_1.jpg]

[image/jpeg:Nucleus Copyright.jpg]

[image/jpeg:si55551568_40018_1.jpg] 


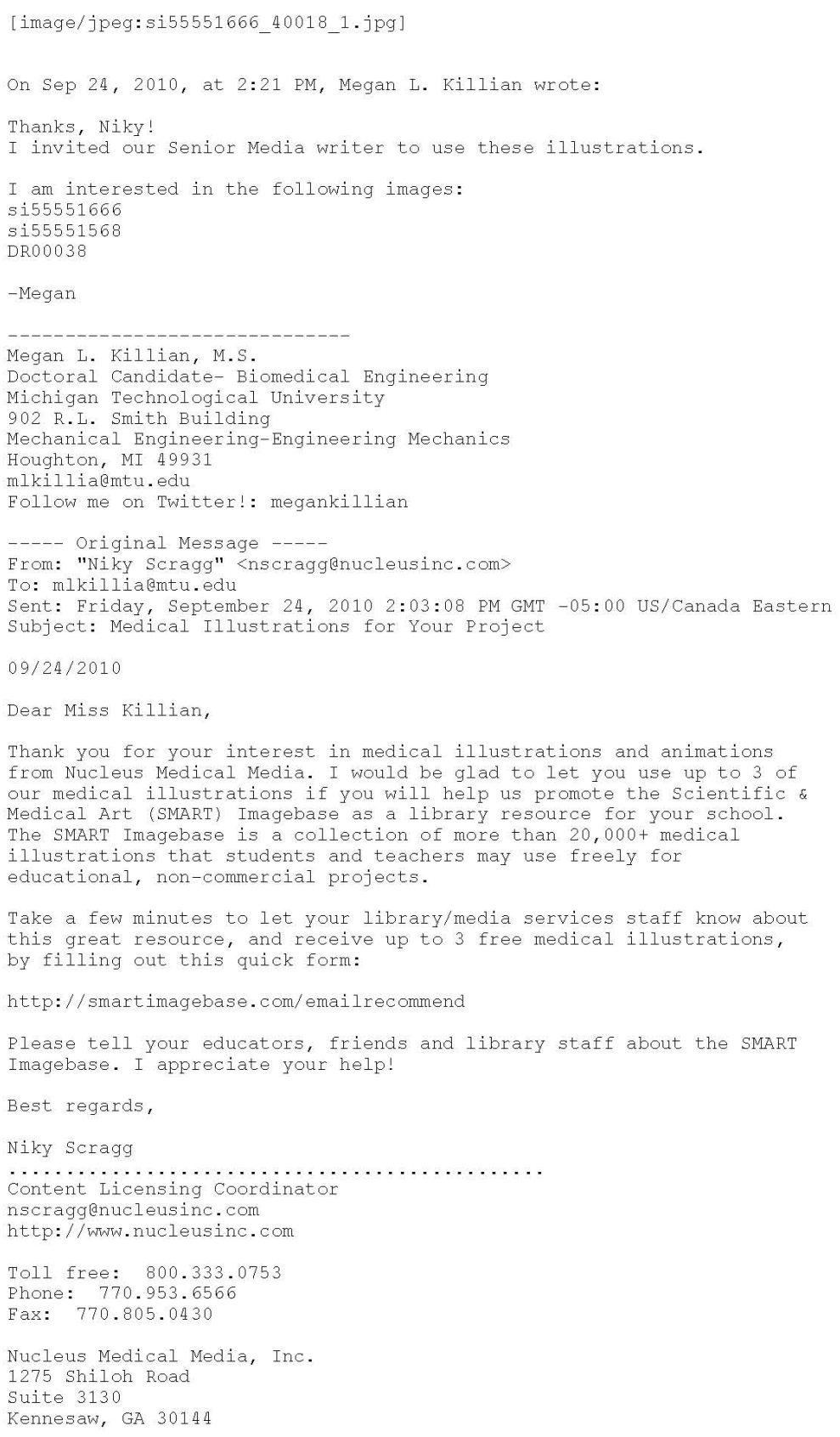




\section{Copyright permission (Figure 1-2)}

\section{JOHN WILEY AND SONS LICENSE}

TERMS AND CONDITIONS

This is a License Agreement between Megan L Killian ("You") and John Wiley and Sons ("John Wiley and Sons") provided by Copyright Clearance Center ("CCC"). The license consists of your order details, the terms and conditions provided by John Wiley and Sons, and the payment terms and conditions.

\begin{tabular}{|c|c|}
\hline $\begin{array}{l}\text { All payments must be ma } \\
\text { information listed at the }\end{array}$ & $\begin{array}{l}\text { de in full to CCC. For payment instructions, please see } \\
\text { ottom of this form. }\end{array}$ \\
\hline License Number & 2515570980247 \\
\hline License date & Sep 24, 2010 \\
\hline Licensed content publisher & John Wiley and Sons \\
\hline Licensed content publication & Congenital Anomalies \\
\hline Licensed content title & Development of the meniscus of the knee joint in human fetuses \\
\hline Licensed content author & Ikuo Fukazawa,Toshihisa Hatta,Yuji Uchio,Hiroki Otani \\
\hline Licensed content date & Mar 1, 2009 \\
\hline Start page & 27 \\
\hline End page & 32 \\
\hline Type of use & Dissertation/Thesis \\
\hline Requestor type & University/Academic \\
\hline Format & Print and electronic \\
\hline Portion & Figure/table \\
\hline Number of figures/tables & 1 \\
\hline $\begin{array}{l}\text { Original Wiley figure/table } \\
\text { number(s) }\end{array}$ & Figure 1 \\
\hline Will you be translating? & No \\
\hline Order reference number & \\
\hline Total & 0.00 USD \\
\hline Eerms and Conditions & \\
\hline
\end{tabular}

\section{TERMS AND CONDITIONS}

This copyrighted material is owned by or exclusively licensed to John Wiley \& Sons, Inc. or one if its group companies (each a "Wiley Company") or a society for whom a Wiley Company has exclusive publishing rights in relation to a particular journal (collectively "WILEY"). By clicking "accept" in connection with completing this licensing transaction, you agree that the following terms and conditions apply to this transaction (along with the billing and payment terms and conditions established by the Copyright Clearance Center Inc., ("CCC's Billing and Payment terms and conditions"), at the time that you opened your Rightslink account (these are available at any time at http://myaccount.copyright.com).

Terms and Conditions

1. The materials you have requested permission to reproduce (the "Materials") are protected by copyright. 
2. You are hereby granted a personal, non-exclusive, non-sublicensable, non-transferable, worldwide, limited license to reproduce the Materials for the purpose specified in the licensing process. This license is for a one-time use only with a maximum distribution equal to the number that you identified in the licensing process. Any form of republication granted by this licence must be completed within two years of the date of the grant of this licence (although copies prepared before may be distributed thereafter). Any electronic posting of the Materials is limited to one year from the date permission is granted and is on the condition that a link is placed to the journal homepage on Wiley's online journals publication platform at www. interscience. wiley.com. The Materials shall not be used in any other manner or for any other purpose. Permission is granted subject to an appropriate acknowledgement given to the author, title of the material/book/journa and the publisher and on the understanding that nowhere in the text is a previously published source acknowledged for all or part of this Material. Any third party material is expressly excluded from this permission.

3. With respect to the Materials, all rights are reserved. No part of the Materials may be copied, modified, adapted, translated, reproduced, transferred or distributed, in any form or by any means, and no derivative works may be made based on the Materials without the prior permission of the respective copyright owner. You may not alter, remove or suppress in any manner any copyright, trademark or other notices displayed by the Materials. You may not license, rent, sell, loan, lease, pledge, offer as security, transfer or assign the Materials, or any of the rights granted to you hereunder to any other person.

4. The Materials and all of the intellectual property rights therein shall at all times remain the exclusive property of John Wiley \& Sons Inc or one of its related companies (WILEY) or their respective licensors, and your interest therein is only that of having possession of and the right to reproduce the Materials pursuant to Section 2 herein during the continuance of this Agreement. You agree that you own no right, title or interest in or to the Materials or any of the intellectual property rights therein. You shall have no rights hereunder other than the license as provided for above in Section 2. No right, license or interest to any trademark, trade name, service mark or other branding ("Marks") of WILEY or its licensors is granted hereunder, and you agree that you shall not assert any such right, license or interest with respect thereto.

5. WILEY DOES NOT MAKE ANY WARRANTY OR REPRESENTATION OF ANY KIND TO YOU OR ANY THIRD PARTY, EXPRESS, IMPLIED OR STATUTORY, WITH RESPECT TO THE MATERIALS OR THE ACCURACY OF ANY INFORMATION CONTAINED IN THE MATERIALS, INCLUDING, WITHOUT LIMITATION, ANY IMPLIED WARRANTY OF MERCHANTABILITY, ACCURACY, SATISFACTORY QUALITY, FITNESS FOR A PARTICULAR PURPOSE, USABILITY, INTEGRATIÓN OR NON-INFRINGEMENT AND ALL SUCH WARRANTIES ARE HEREBY EXCLUDED BY WILEY AND WAIVED BY YOU.

6. WILEY shall have the right to terminate this Agreement immediately upon breach of this Agreement by you.

7. You shall indemnify, defend and hold harmless WILEY, its directors, officers, agents and employees, from and against any actual or threatened claims, demands, causes of action or proceedings arising from any breach of this Agreement by you.

8. IN NO EVENT SHALL WILEY BE LIABLE TO YOU OR ANY OTHER PARTY OR ANY OTHER PERSON OR ENTITY FOR ANY SPECIAL, CONSEQUENTIAL, INCIDENTAL, INDIRECT, EXEMPLARY OR PUNITIVE DAMAGES, HOWEVER CAUSED, ARISING OUT OF OR IN CONNECTION WITH THE DOWNLOADING, PROVISIONING, VIEWING OR USE OF THE MATERIALS REGARDLESS OF THE FORM OF ACTION, WHETHER FOR BREACH OF CONTRACT, BREACH OF WARRANTY, TORT, NEGLIGENCE, INFRINGEMENT OR OTHERWISE (INCLUDING, WITHOUT LIMITATION, DAMAGES BASED ON LOSS OF PROFITS, DATA, FILES, USE, BUSINESS OPPORTUNITY OR CLAIMS OF THIRD PARTIES), AND WHETHER OR NOT THE PARTY HAS BEEN ADVISED OF THE POSSIBILITY OF SUCH DAMAGES. THIS LIMITATION SHALL APPLY NOTWITHSTANDING ANY FAILURE OF ESSENTIAL PURPOSE OF ANY LIMITED REMEDY PROVIDED HEREIN.

9. Should any provision of this Agreement be held by a court of competent jurisdiction to be illegal, invalid, or unenforceable, that provision shall be deemed amended to achieve as nearly as possible the same economic effect as the original provision, and the legality, validity and enforceability of the remaining provisions of this Agreement shall not be affected or impaired thereby. 
10. The failure of either party to enforce any term or condition of this Agreement shall not constitute a waiver of either party's right to enforce each and every term and condition of this Agreement. No breach under this agreement shall be deemed waived or excused by either party unless such waiver or consent is in writing signed by the party granting such waiver or consent. The waiver by or consent of a party to a breach of any provision of this Agreement shall not operate or be construed as a waiver of or consent to any other or subsequent breach by such other party.

11. This Agreement may not be assigned (including by operation of law or otherwise) by you without WILEY's prior written consent.

12. These terms and conditions together with CCC's Billing and Payment terms and conditions (which are incorporated herein) form the entire agreement between you and WILEY concerning this licensing transaction and (in the absence of fraud) supersedes all prior agreements and representations of the parties, oral or written. This Agreement may not be amended except in a writing signed by both parties. This Agreement shall be binding upon and inure to the benefit of the parties' successors, legal representatives, and authorized assigns.

13. In the event of any conflict between your obligations established by these terms and conditions and those established by CCC's Billing and Payment terms and conditions, these terms and conditions shall prevail.

14. WILEY expressly reserves all rights not specifically granted in the combination of (i) the license details provided by you and accepted in the course of this licensing transaction, (ii) these terms and conditions and (iii) CCC's Billing and Payment terms and conditions.

15. This Agreement shall be governed by and construed in accordance with the laws of England and you agree to submit to the exclusive jurisdiction of the English courts.

16. Other Terms and Conditions:

BY CLICKING ON THE "I ACCEPT" BUTTON, YOU ACKNOWLEDGE THAT YOU HAVE READ AND FULLY UNDERSTAND EACH OF THE SECTIONS OF AND PROVISIONS SET FORTH IN THIS AGREEMENT AND THAT YOU ARE IN AGREEMENT WITH AND ARE WILLING TO ACCEPT ALL OF YOUR OBLIGATIONS AS SET FORTH IN THIS AGREEMENT.

V1.2

Gratis licenses (referencing $\$ 0$ in the Total field) are free. Please retain this printable license for your reference. No payment is required.

If you would like to pay for this license now, please remit this license along with your payment made payable to "COPYRIGHT CLEARANCE CENTER" otherwise you will be invoiced within $\mathbf{4 8}$ hours of the license date. Payment should be in the form of a check or money order referencing your account number and this invoice number RLNK10855027.

Once you receive your invoice for this order, you may pay your invoice by credit card. Please follow instructions provided at that time.

Make Payment To:

Copyright Clearance Center

Dept 001

P.O. Box 843006

Boston, MA 02284-3006

If you find copyrighted material related to this license will not be used and wish to cancel, please contact us referencing this license number 2515570980247 and noting the reason for cancellation.

Questions? customercare@copvriaht.com or +1-877-622-5543 (toll free in the US) or +1-978-646-2777. 


\title{
Copyright permission ( Figure 1-3)
}

RE: Copyright request for non-commercially published dissertation

From: brocheleau@rockwaterinc.com

To: mlkillia@mtu.edu

Dear Ms. Killian,

JBJS figures may be used in a dissertation as long as the material is not modified, JBJS

is properly credited, and the dissertation is not commercially published. If it is later

commercially published it will be necessary to submit a new request.

Kind regards,

Beth Ann Rocheleau

Intellectual Froperty Manager

Rockwater, Inc.

PH $803 / 359-4578$

FX $803 / 753-9430$

brocheleaulockwaterinc.com

-----Original Message-----

From: Megan L. Killian [mailto:mlkillia@mtu.edu]

Sent: Saturday, September 25, 2010 10:12 AM

To: jbjsdrockwaterinc.com

Subject: Copyright request for non-commercially published dissertation

Greetings -

I would like to request use of figures from Clark et al 1983 (Journal of Bone and Joint

Surgery) to be used in my doctoral dissertation at Michigan Technological University.

Please see the attached copyright request letter. Feel free to email me or call at

906-231-9248 if you have any questions.

Thank you,

Megan

Megan L. Killian, M.S.

Doctoral Candidate- Biomedical Engineering Michigan Technological University

902 R. L. Smith Building

Mechanical Engineering-Engineering Mechanics Houghton, MI 49931 mlkillia@mtu.edu

Follow me on Twitter!: megankillian 


\title{
Copyright permission (Figure 1-5)
}

\author{
Rightslink Printable License
}

https://s100.copyright.com/App/PrintableLicenseFrame.jsp?publisherID..

\begin{abstract}
ELSEVIER LICENSE
TERMS AND CONDITIONS
\end{abstract}

Sep 26, 2010

This is a License Agreement between Megan L Killian ("You") and Elsevier ("Elsevier") provided by Copyright Clearance Center ("CCC"). The license consists of your order details, the terms and conditions provided by Elsevier, and the payment terms and conditions.

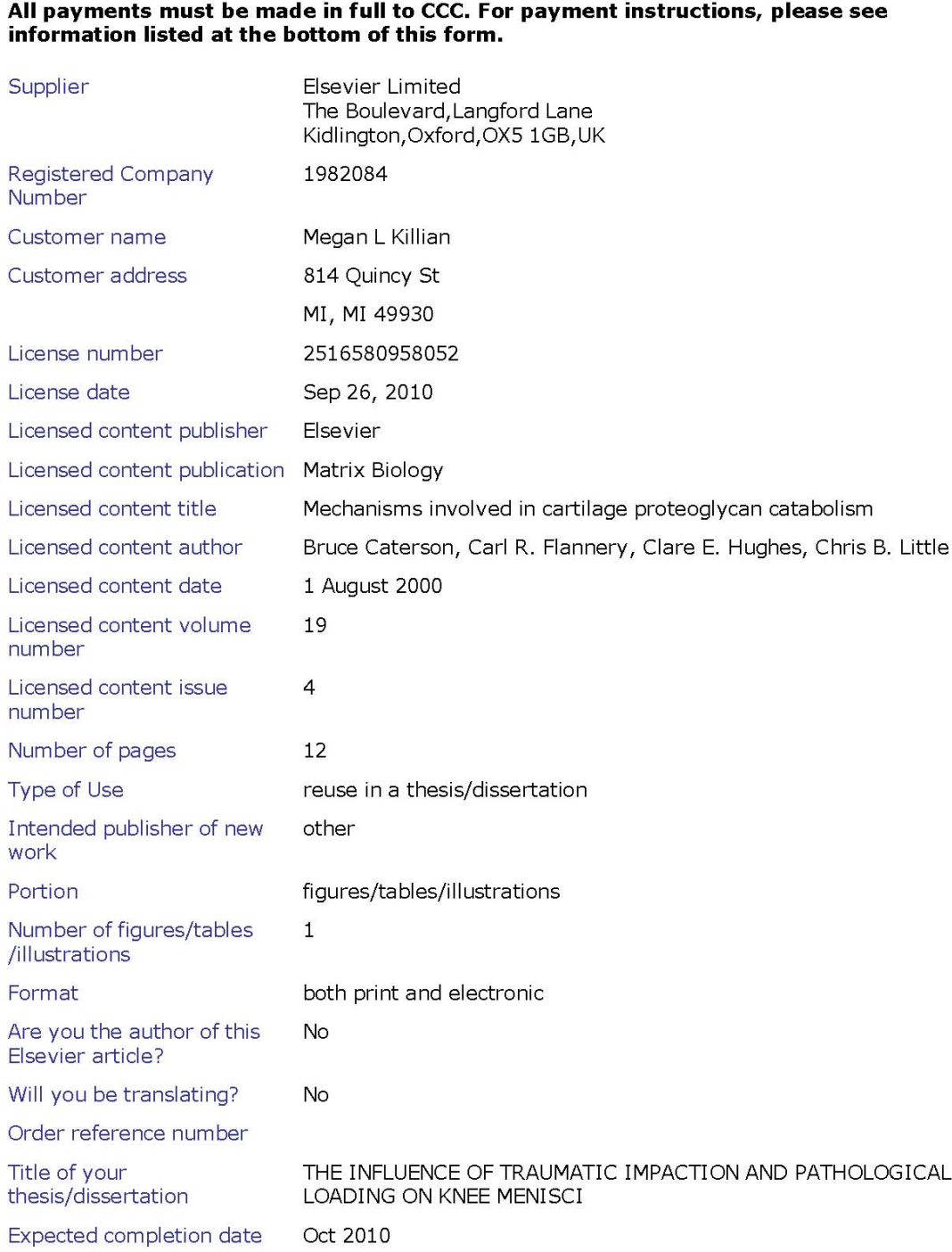




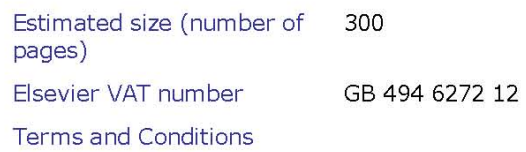

\section{INTRODUCTION}

1. The publisher for this copyrighted material is Elsevier. By clicking "accept" in connection with completing this licensing transaction, you agree that the following terms and conditions apply to this transaction (along with the Billing and Payment terms and conditions established by Copyright Clearance Center, Inc. ("CCC"), at the time that you opened your Rightslink account and that are available at any time at http://myaccount.copyright.com).

\section{GENERAL TERMS}

2. Elsevier hereby grants you permission to reproduce the aforementioned material subject to the terms and conditions indicated.

3. Acknowledgement: If any part of the material to be used (for example, figures) has appeared in our publication with credit or acknowledgement to another source, permission must also be sought from that source. If such permission is not obtained then that material may not be included in your publication/copies. Suitable acknowledgement to the source must be made, either as a footnote or in a reference list at the end of your publication, as follows:

"Reprinted from Publication title, Vol/edition number, Author(s), Title of article / title of chapter, Pages No., Copyright (Year), with permission from Elsevier [OR APPLICABLE SOCIETY COPYRIGHT OWNER]." Also Lancet special credit - "Reprinted from The Lancet, Vol. number, Author(s), Title of article, Pages No., Copyright (Year), with permission from Elsevier."

4. Reproduction of this material is confined to the purpose and/or media for which permission is hereby given.

5. Altering/Modifying Material: Not Permitted. However figures and illustrations may be altered/adapted minimally to serve your work. Any other abbreviations, additions, deletions and/or any other alterations shall be made only with prior written authorization of Elsevier Ltd. (Please contact Elsevier at permissions@elsevier.com)

6. If the permission fee for the requested use of our material is waived in this instance, please be advised that your future requests for Elsevier materials may attract a fee.

7. Reservation of Rights: Publisher reserves all rights not specifically granted in the combination of (i) the license details provided by you and accepted in the course of this licensing transaction, (ii) these terms and conditions and (iii) CCC's Billing and Payment terms and conditions.

8. License Contingent Upon Payment: While you may exercise the rights licensed immediately upon issuance of the license at the end of the licensing process for the transaction, provided that you have disclosed complete and accurate details of your proposed use, no license is finally effective unless and until full payment is received from you (either by publisher or by $\mathrm{CCC}$ ) as provided in CCC's Billing and Payment terms and 
conditions. If full payment is not received on a timely basis, then any license preliminarily granted shall be deemed automatically revoked and shall be void as if never granted. Further, in the event that you breach any of these terms and conditions or any of CCC's Billing and Payment terms and conditions, the license is automatically revoked and shall be void as if never granted. Use of materials as described in a revoked license, as well as any use of the materials beyond the scope of an unrevoked license, may constitute copyright infringement and publisher reserves the right to take any and all action to protect its copyright in the materials.

9. Warranties: Publisher makes no representations or warranties with respect to the licensed material.

10. Indemnity: You hereby indemnify and agree to hold harmless publisher and CCC, and their respective officers, directors, employees and agents, from and against any and all claims arising out of your use of the licensed material other than as specifically authorized pursuant to this license.

11. No Transfer of License: This license is personal to you and may not be sublicensed, assigned, or transferred by you to any other person without publisher's written permission.

12. No Amendment Except in Writing: This license may not be amended except in a writing signed by both parties (or, in the case of publisher, by CCC on publisher's behalf).

13. Objection to Contrary Terms: Publisher hereby objects to any terms contained in any purchase order, acknowledgment, check endorsement or other writing prepared by you, which terms are inconsistent with these terms and conditions or CCC's Billing and Payment terms and conditions. These terms and conditions, together with CCC's Billing and Payment terms and conditions (which are incorporated herein), comprise the entire agreement between you and publisher (and CCC) concerning this licensing transaction. In the event of any conflict between your obligations established by these terms and conditions and those established by CCC's Billing and Payment terms and conditions, these terms and conditions shall control.

14. Revocation: Elsevier or Copyright Clearance Center may deny the permissions described in this License at their sole discretion, for any reason or no reason, with a full refund payable to you. Notice of such denial will be made using the contact information provided by you. Failure to receive such notice will not alter or invalidate the denial. In no event will Elsevier or Copyright Clearance Center be responsible or liable for any costs, expenses or damage incurred by you as a result of a denial of your permission request, other than a refund of the amount(s) paid by you to Elsevier and/or Copyright Clearance Center for denied permissions.

\section{LIMITED LICENSE}

The following terms and conditions apply only to specific license types:

15. Translation: This permission is granted for non-exclusive world English rights only unless your license was granted for translation rights. If you licensed translation rights you may only translate this content into the languages you requested. A professional translator must perform all translations and reproduce the content word for word preserving the integrity of the article. If this license is to re-use 1 or 2 figures then permission is granted for non-exclusive world rights in all languages. 
16. Website: The following terms and conditions apply to electronic reserve and author websites:

Electronic reserve: If licensed material is to be posted to website, the web site is to be password-protected and made available only to bona fide students registered on a relevant course if:

This license was made in connection with a course,

This permission is granted for 1 year only. You may obtain a license for future website posting,

All content posted to the web site must maintain the copyright information line on the bottom of each image,

A hyper-text must be included to the Homepage of the journal from which you are licensing at http://www.sciencedirect.com/science/journal/xxxxx or the Elsevier homepage for books at http://www.elsevier.com, and

Central Storage: This license does not include permission for a scanned version of the material to be stored in a central repository such as that provided by Heron/XanEdu.

17. Author website for journals with the following additional clauses:

All content posted to the web site must maintain the copyright information line on the bottom of each image, and

he permission granted is limited to the personal version of your paper. You are not allowed to download and post the published electronic version of your article (whether PDF or HTML, proof or final version), nor may you scan the printed edition to create an electronic version,

A hyper-text must be included to the Homepage of the journal from which you are licensing at http://www.sciencedirect.com/science/journal/xxxxx , As part of our normal production process, you will receive an e-mail notice when your article appears on Elsevier's online service ScienceDirect (www.sciencedirect.com). That e-mail will include the article's Digital Object Identifier (DOI). This number provides the electronic link to the published article and should be included in the posting of your personal version. We ask that you wait until you receive this e-mail and have the DOI to do any posting.

Central Storage: This license does not include permission for a scanned version of the material to be stored in a central repository such as that provided by Heron/XanEdu.

18. Author website for books with the following additional clauses: Authors are permitted to place a brief summary of their work online only.

A hyper-text must be included to the Elsevier homepage at http:/www.elsevier.com

All content posted to the web site must maintain the copyright information line on the bottom of each image

You are not allowed to download and post the published electronic version of your chapter, nor may you scan the printed edition to create an electronic version.

Central Storage: This license does not include permission for a scanned version of the material to be stored in a central repository such as that provided by Heron/XanEdu.

19. Website (regular and for author): A hyper-text must be included to the Homepage of the journal from which you are licensing at http://www.sciencedirect.com/science/journal

/xxxxx. or for books to the Elsevier homepage at http://www.elsevier.com 
20. Thesis/Dissertation: If your license is for use in a thesis/dissertation your thesis may be submitted to your institution in either print or electronic form. Should your thesis be published commercially, please reapply for permission. These requirements include permission for the Library and Archives of Canada to supply single copies, on demand, of the complete thesis and include permission for UMI to supply single copies, on demand, of the complete thesis. Should your thesis be published commercially, please reapply for permission.

\section{Other Conditions:}

v1.6

Gratis licenses (referencing $\$ 0$ in the Total field) are free. Please retain this printable license for your reference. No payment is required.

If you would like to pay for this license now, please remit this license along with your payment made payable to "COPYRIGHT CLEARANCE CENTER" otherwise you will be invoiced within 48 hours of the license date. Payment should be in the form of a check or money order referencing your account number and this invoice number RLNK10855253.

Once you receive your invoice for this order, you may pay your invoice by credit card. Please follow instructions provided at that time.

Make Payment To:

Copyright Clearance Center

Dept 001

P.O. Box 843006

Boston, MA 02284-3006

If you find copyrighted material related to this license will not be used and wish to cancel, please contact us referencing this license number 2516580958052 and noting the reason for cancellation.

Questions? customercare@copvright.com or +1-877-622-5543 (toll free in the US) or +1-978-646-2777. 


\title{
Copyright permission (Figure 1-8)
}

\author{
Rightslink Printable License
}

\section{JOHN WILEY AND SONS LICENSE TERMS AND CONDITIONS}

This is a License Agreement between Megan L Killian ("You") and John Wiley and Sons ("John Wiley and Sons") provided by Copyright Clearance Center ("CCC"). The license consists of your order details, the terms and conditions provided by John Wiley and Sons, and the payment terms and conditions

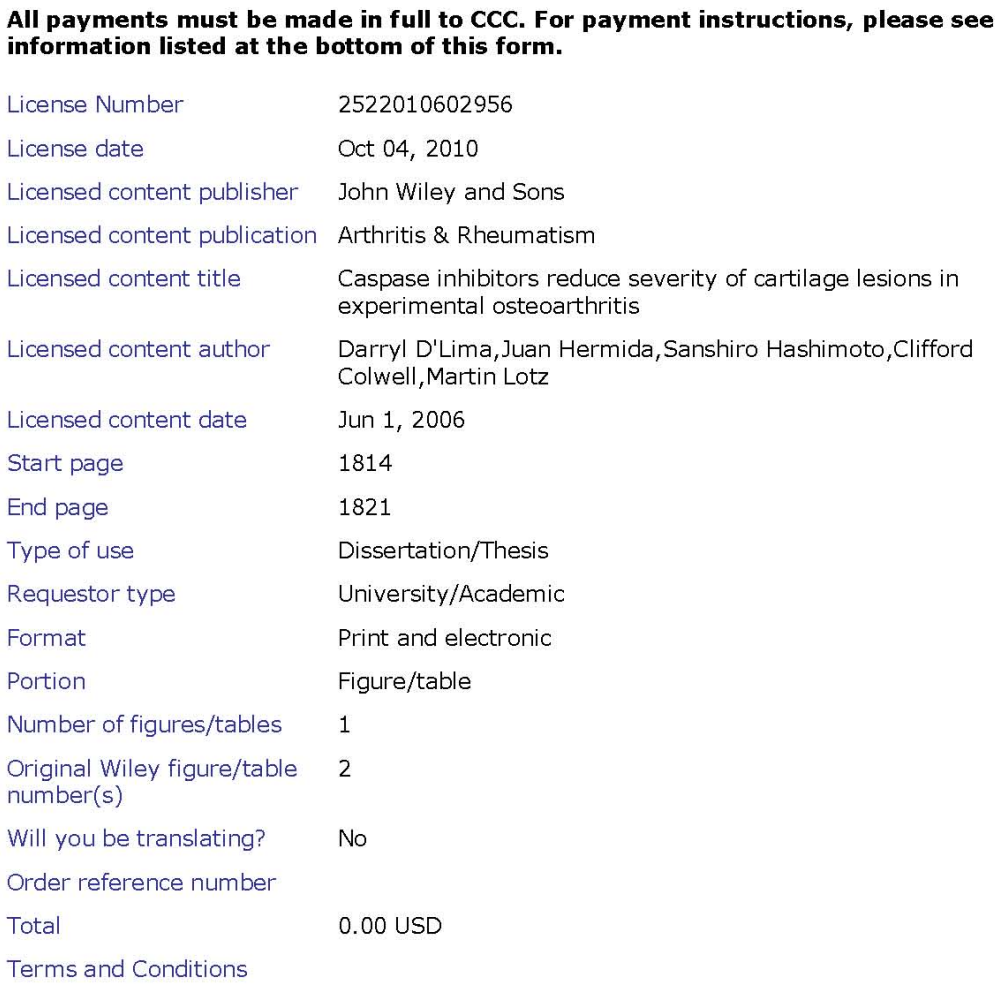

Terms and Conditions

\section{TERMS AND CONDITIONS}

This copyrighted material is owned by or exclusively licensed to John Wiley \& Sons, Inc. or one if its group companies (each a "Wiley Company") or a society for whom a Wiley Company has exclusive publishing rights in relation to a particular journal (collectively "WILEY"). By clicking "accept" in connection with completing this licensing transaction, you agree that the following terms and conditions apply to this transaction (along with the billing and payment terms and conditions established by the Copyright Clearance Center Inc., ("CCC's Billing and Payment terms and conditions"), at the time that you opened your Rightslink account (these are available at any time at http://myaccount.copyright.com).

Terms and Conditions

1. The materials you have requested permission to reproduce (the "Materials") are protected by 
copyright.

2. You are hereby granted a personal, non-exclusive, non-sublicensable, non-transferable, worldwide, limited license to reproduce the Materials for the purpose specified in the licensing process. This license is for a one-time use only with a maximum distribution equal to the number that you identified in the licensing process. Any form of republication granted by this licence must be completed within two years of the date of the grant of this licence (although copies prepared before may be distributed thereafter). Any electronic posting of the Materials is limited to one year from the date permission is granted and is on the condition that a link is placed to the journal homepage on Wiley's online journals publication platform at www. interscience. wiley.com. The Materials shall not be used in any other manner or for any other purpose. Permission is granted subject to an appropriate acknowledgement given to the author, title of the material/book/journal and the publisher and on the understanding that nowhere in the text is a previously published source acknowledged for all or part of this Material. Any third party material is expressly excluded from this permission.

3. With respect to the Materials, all rights are reserved. No part of the Materials may be copied, modified, adapted, translated, reproduced, transferred or distributed, in any form or by any means, and no derivative works may be made based on the Materials without the prior permission of the respective copyright owner. You may not alter, remove or suppress in any manner any copyright, trademark or other notices displayed by the Materials. You may not license, rent, sell, loan, lease, pledge, offer as security, transfer or assign the Materials, or any of the rights granted to you hereunder to any other person.

4. The Materials and all of the intellectual property rights therein shall at all times remain the exclusive property of John Wiley \& Sons Inc or one of its related companies (WILEY) or their respective licensors, and your interest therein is only that of having possession of and the right to reproduce the Materials pursuant to Section 2 herein during the continuance of this Agreement. You agree that you own no right, title or interest in or to the Materials or any of the intellectual property rights therein. You shall have no rights hereunder other than the license as provided for above in Section 2. No right, license or interest to any trademark, trade name, service mark or other branding ("Marks") of WILEY or its licensors is granted hereunder, and you agree that you shall not assert any such right, license or interest with respect thereto.

5. WILEY DOES NOT MAKE ANY WARRANTY OR REPRESENTATION OF ANY KIND TO YOU OR ANY THIRD PARTY, EXPRESS, IMPLIED OR STATUTORY, WITH RESPECT TO THE MATERIALS OR THE ACCURACY OF ANY INFORMATION CONTAINED IN THE MATERIALS, INCLUDING, WITHOUT LIMITATION, ANY IMPLIED WARRANTY OF MERCHANTABILITY, ACCURACY, SATISFACTORY QUALITY, FITNESS FOR A PARTICULAR PURPOSE, USABILITY, INTEGRATION OR NON-INFRINGEMENT AND ALL SUCH WARRANTIES ARE HEREBY EXCLUDED BY WILEY AND WAIVED BY YOU.

6. WILEY shall have the right to terminate this Agreement immediately upon breach of this Agreement by you.

7. You shall indemnify, defend and hold harmless WILEY, its directors, officers, agents and employees, from and against any actual or threatened claims, demands, causes of action or proceedings arising from any breach of this Agreement by you.

8. IN NO EVENT SHALL WILEY BE LIABLE TO YOU OR ANY OTHER PARTY OR ANY OTHER PERSON OR ENTITY FOR ANY SPECIAL, CONSEOUENTIAL, INCIDENTAL, INDIRECT, EXEMPLARY OR PUNITIVE DAMAGES, HOWEVER CAUSED, ARISING OUT OF OR IN CONNECTION WITH THE DOWNLOADING, PROVISIONING, VIEWING OR USE OF THE MATERIALS REGARDLESS OF THE FORM OF ACTION, WHETHER FOR BREACH OF CONTRACT, BREACH OF WARRANTY, TORT, NEGLIGENCE, INFRINGEMENT OR OTHERWISE (INCLUDING, WITHOUT LIMITATION, DAMAGES BASED ON LOSS OF PROFITS, DATA, FILES, USE, BUSINESS OPPORTUNITY OR CLAIMS OF THIRD PARTIES), AND WHETHER OR NOT THE PARTY HAS BEEN ADVISED OF THE POSSIBILITY OF SUCH DAMAGES. THIS LIMITATION SHALL APPLY NOTWITHSTANDING ANY FAILURE OF ESSENTIAL PURPOSE OF ANY LIMITED REMEDY PROVIDED HEREIN.

9. Should any provision of this Agreement be held by a court of competent jurisdiction to be illegal, invalid, or unenforceable, that provision shall be deemed amended to achieve as nearly as possible the same economic effect as the original provision, and the legality, validity and enforceability of the remaining provisions of this Agreement shall not be affected or impaired 
thereby.

10. The failure of either party to enforce any term or condition of this Agreement shall not constitute a waiver of either party's right to enforce each and every term and condition of this Agreement. No breach under this agreement shall be deemed waived or excused by either party unless such waiver or consent is in writing signed by the party granting such waiver or consent. The waiver by or consent of a party to a breach of any provision of this Agreement shall not operate or be construed as a waiver of or consent to any other or subsequent breach by such other party.

11. This Agreement may not be assigned (including by operation of law or otherwise) by you without WILEY's prior written consent.

12. These terms and conditions together with CCC's Billing and Payment terms and conditions (which are incorporated herein) form the entire agreement between you and WILEY concerning this licensing transaction and (in the absence of fraud) supersedes all prior agreements and representations of the parties, oral or written. This Agreement may not be amended except in a writing signed by both parties. This Agreement shall be binding upon and inure to the benefit of the parties' successors, legal representatives, and authorized assigns.

13. In the event of any conflict between your obligations established by these terms and conditions and those established by CCC's Billing and Payment terms and conditions, these terms and conditions shall prevail.

14. WILEY expressly reserves all rights not specifically granted in the combination of (i) the license details provided by you and accepted in the course of this licensing transaction, (ii) these terms and conditions and (iii) CCC's Billing and Payment terms and conditions.

15. This Agreement shall be governed by and construed in accordance with the laws of England and you agree to submit to the exclusive jurisdiction of the English courts.

16. Other Terms and Conditions:

BY CLICKING ON THE "I ACCEPT" BUTTON, YOU ACKNOWLEDGE THAT YOU HAVE READ AND FULLY UNDERSTAND EACH OF THE SECTIONS OF AND PROVISIONS SET FORTH IN THIS AGREEMENT AND THAT YOU ARE IN AGREEMENT WITH AND ARE WILLING TO ACCEPT ALL OF YOUR OBLIGATIONS AS SET FORTH IN THIS AGREEMENT.

V1.2

Gratis licenses (referencing $\$ 0$ in the Total field) are free. Please retain this printable license for your reference. No payment is required.

If you would like to pay for this license now, please remit this license along with your payment made payable to "COPYRIGHT CLEARANCE CENTER" otherwise you will be invoiced within $\mathbf{4 8}$ hours of the license date. Payment should be in the form of a check or money order referencing your account number and this invoice number RLNK10860105.

Once you receive your invoice for this order, you may pay your invoice by credit card. Please follow instructions provided at that time.

Make Payment To:

Copyright Clearance Center

Dept 001

P.o. Box 843006

Boston, MA 02284-3006

If you find copyrighted material related to this license will not be used and wish to cancel, please contact us referencing this license number 2522010602956 and noting the reason for cancellation.

Questions? customercare@copvriaht.com or $+1-877-622-5543$ (toll free in the US) or +1-978-646-2777 


\title{
Copyright permission (Figure 1-8)
}

\author{
Rightslink Printable License
}

https://s100.copyright.com/App/PrintableLicenseFrame.jsp?publisherID..

\author{
ELSEVIER LICENSE \\ TERMS AND CONDITIONS
}

Oct 19, 2010

This is a License Agreement between Megan L Killian ("You") and Elsevier ("Elsevier") provided by Copyright Clearance Center ("CCC"). The license consists of your order details, the terms and conditions provided by Elsevier, and the payment terms and conditions.

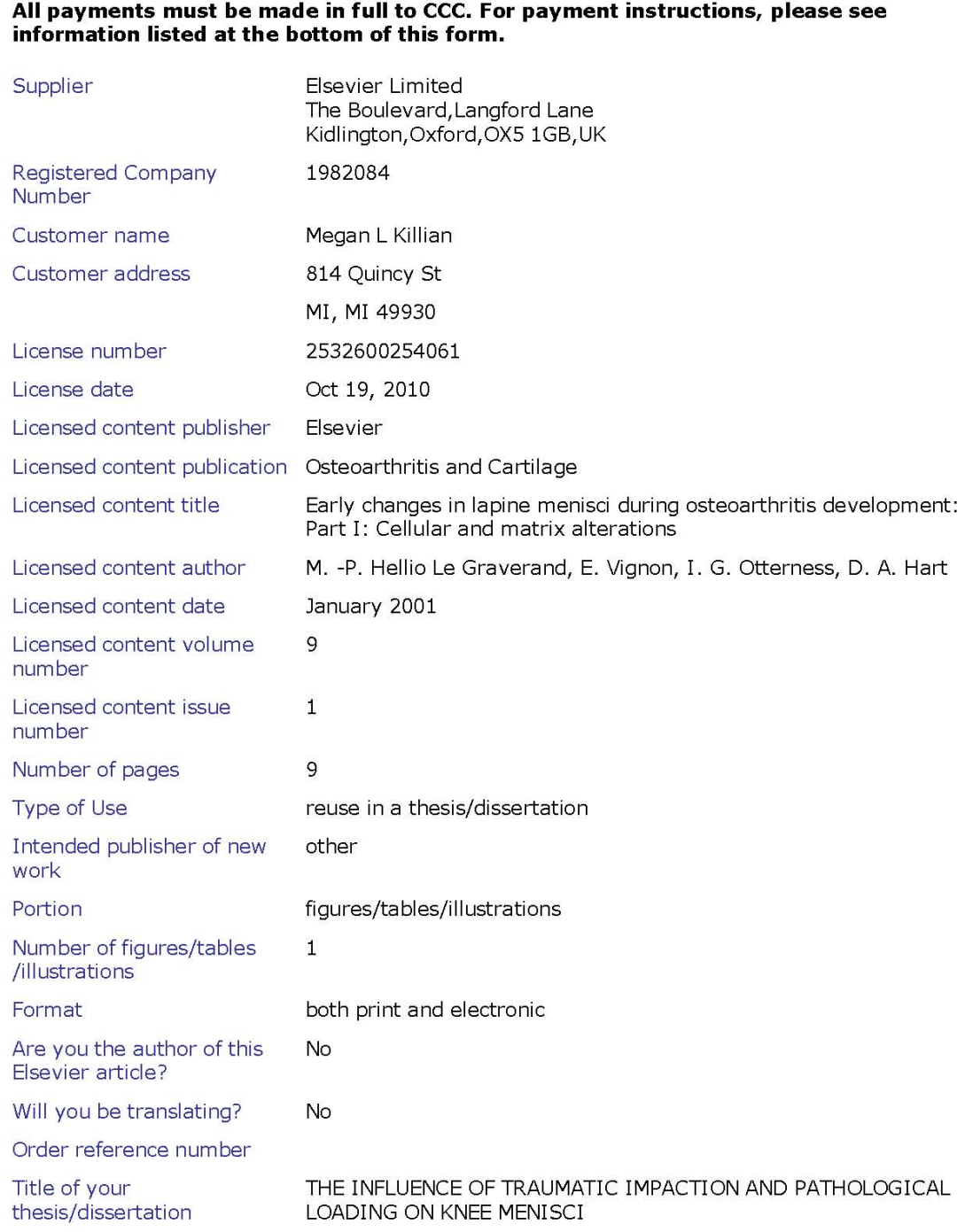




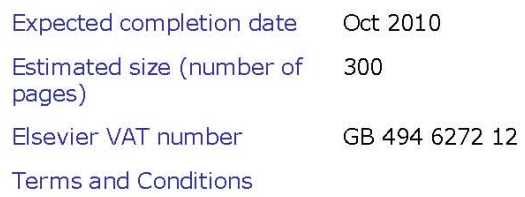

\section{INTRODUCTION}

1. The publisher for this copyrighted material is Elsevier. By clicking "accept" in connection with completing this licensing transaction, you agree that the following terms and conditions apply to this transaction (along with the Billing and Payment terms and conditions established by Copyright Clearance Center, Inc. ("CCC"), at the time that you opened your Rightslink account and that are available at any time at http://myaccount.copyright.com).

\section{GENERAL TERMS}

2. Elsevier hereby grants you permission to reproduce the aforementioned material subject to the terms and conditions indicated.

3. Acknowledgement: If any part of the material to be used (for example, figures) has appeared in our publication with credit or acknowledgement to another source, permission must also be sought from that source. If such permission is not obtained then that material may not be included in your publication/copies. Suitable acknowledgement to the source must be made, either as a footnote or in a reference list at the end of your publication, as follows:

"Reprinted from Publication title, Vol/edition number, Author(s), Title of article / title of chapter, Pages No., Copyright (Year), with permission from Elsevier [OR APPLICABLE SOCIETY COPYRIGHT OWNER]." Also Lancet special credit - "Reprinted from The Lancet, Vol. number, Author(s), Title of article, Pages No., Copyright (Year), with permission from Elsevier."

4. Reproduction of this material is confined to the purpose and/or media for which permission is hereby given.

5. Altering/Modifying Material: Not Permitted. However figures and illustrations may be altered/adapted minimally to serve your work. Any other abbreviations, additions, deletions and/or any other alterations shall be made only with prior written authorization of Elsevier Ltd. (Please contact Elsevier at permissions@elsevier.com)

6. If the permission fee for the requested use of our material is waived in this instance, please be advised that your future requests for Elsevier materials may attract a fee.

7. Reservation of Rights: Publisher reserves all rights not specifically granted in the combination of (i) the license details provided by you and accepted in the course of this licensing transaction, (ii) these terms and conditions and (iii) CCC's Billing and Payment terms and conditions.

8. License Contingent Upon Payment: While you may exercise the rights licensed immediately upon issuance of the license at the end of the licensing process for the transaction, provided that you have disclosed complete and accurate details of your 
proposed use, no license is finally effective unless and until full payment is received from you (either by publisher or by CCC) as provided in CCC's Billing and Payment terms and conditions. If full payment is not received on a timely basis, then any license preliminarily granted shall be deemed automatically revoked and shall be void as if never granted. Further, in the event that you breach any of these terms and conditions or any of CCC's Billing and Payment terms and conditions, the license is automatically revoked and shall be void as if never granted. Use of materials as described in a revoked license, as well as any use of the materials beyond the scope of an unrevoked license, may constitute copyright infringement and publisher reserves the right to take any and all action to protect its copyright in the materials.

9. Warranties: Publisher makes no representations or warranties with respect to the licensed material.

10. Indemnity: You hereby indemnify and agree to hold harmless publisher and CCC, and their respective officers, directors, employees and agents, from and against any and all claims arising out of your use of the licensed material other than as specifically authorized pursuant to this license.

11. No Transfer of License: This license is personal to you and may not be sublicensed, assigned, or transferred by you to any other person without publisher's written permission.

12. No Amendment Except in Writing: This license may not be amended except in a writing signed by both parties (or, in the case of publisher, by CCC on publisher's behalf).

13. Objection to Contrary Terms: Publisher hereby objects to any terms contained in any purchase order, acknowledgment, check endorsement or other writing prepared by you, which terms are inconsistent with these terms and conditions or CCC's Billing and Payment terms and conditions. These terms and conditions, together with CCC's Billing and Payment terms and conditions (which are incorporated herein), comprise the entire agreement between you and publisher (and $\mathrm{CCC}$ ) concerning this licensing transaction. In the event of any conflict between your obligations established by these terms and conditions and those established by CCC's Billing and Payment terms and conditions, these terms and conditions shall control.

14. Revocation: Elsevier or Copyright Clearance Center may deny the permissions described in this License at their sole discretion, for any reason or no reason, with a full refund payable to you. Notice of such denial will be made using the contact information provided by you. Failure to receive such notice will not alter or invalidate the denial. In no event will Elsevier or Copyright Clearance Center be responsible or liable for any costs, expenses or damage incurred by you as a result of a denial of your permission request, other than a refund of the amount(s) paid by you to Elsevier and/or Copyright Clearance Center for denied permissions.

\section{LIMITED LICENSE}

The following terms and conditions apply only to specific license types:

15. Translation: This permission is granted for non-exclusive world English rights only unless your license was granted for translation rights. If you licensed translation rights you may only translate this content into the languages you requested. A professional translator must perform all translations and reproduce the content word for word preserving the 
integrity of the article. If this license is to re-use 1 or 2 figures then permission is granted for non-exclusive world rights in all languages.

16. Website: The following terms and conditions apply to electronic reserve and author websites:

Electronic reserve: If licensed material is to be posted to website, the web site is to be password-protected and made available only to bona fide students registered on a relevant course if:

This license was made in connection with a course,

This permission is granted for 1 year only. You may obtain a license for future website posting,

All content posted to the web site must maintain the copyright information line on the bottom of each image,

A hyper-text must be included to the Homepage of the journal from which you are licensing at http:/www.sciencedirect.com/science/journal/xxxxx or the Elsevier homepage for books at http://www.elsevier.com, and

Central Storage: This license does not include permission for a scanned version of the material to be stored in a central repository such as that provided by Heron/XanEdu.

17. Author website for journals with the following additional clauses:

All content posted to the web site must maintain the copyright information line on the bottom of each image, and

he permission granted is limited to the personal version of your paper. You are not allowed to download and post the published electronic version of your article (whether PDF or HTML, proof or final version), nor may you scan the printed edition to create an electronic version,

A hyper-text must be included to the Homepage of the journal from which you are licensing at $\mathrm{http} / /$ www.sciencedirect.com/science/journal/xxxxx , As part of our normal production process, you will receive an e-mail notice when your article appears on Elsevier's online service ScienceDirect (www.sciencedirect.com). That e-mail will include the article's Digital Object Identifier (DOI). This number provides the electronic link to the published article and should be included in the posting of your personal version. We ask that you wait until you receive this e-mail and have the DOI to do any posting.

Central Storage: This license does not include permission for a scanned version of the material to be stored in a central repository such as that provided by Heron/XanEdu.

18. Author website for books with the following additional clauses:

Authors are permitted to place a brief summary of their work online only.

A hyper-text must be included to the Elsevier homepage at http://www.elsevier.com

All content posted to the web site must maintain the copyright information line on the bottom of each image

You are not allowed to download and post the published electronic version of your chapter, nor may you scan the printed edition to create an electronic version.

Central Storage: This license does not include permission for a scanned version of the material to be stored in a central repository such as that provided by Heron/XanEdu.

19. Website (regular and for author): A hyper-text must be included to the Homepage of the 
journal from which you are licensing at http:/www.sciencedirect.com/science/journal /xxxxx. or for books to the Elsevier homepage at http://www.elsevier.com

20. Thesis/Dissertation: If your license is for use in a thesis/dissertation your thesis may be submitted to your institution in either print or electronic form. Should your thesis be published commercially, please reapply for permission. These requirements include permission for the Library and Archives of Canada to supply single copies, on demand, of the complete thesis and include permission for UMI to supply single copies, on demand, of the complete thesis. Should your thesis be published commercially, please reapply for permission.

\section{Other Conditions:}

v1.6

Gratis licenses (referencing $\$ 0$ in the Total field) are free. Please retain this printable license for your reference. No payment is required.

If you would like to pay for this license now, please remit this license along with your payment made payable to "COPYRIGHT CLEARANCE CENTER" otherwise you will be invoiced within $\mathbf{4 8}$ hours of the license date. Payment should be in the form of a check or money order referencing your account number and this invoice number

RLNK10868810.

Once you receive your invoice for this order, you may pay your invoice by credit card. Please follow instructions provided at that time.

Make Payment To:

Copyright Clearance Center

Dept 001

P.O. Box 843006

Boston, MA 02284-3006

If you find copyrighted material related to this license will not be used and wish to cancel, please contact us referencing this license number 2532600254061 and noting the reason for cancellation.

Questions? customercare@copyright.com or +1-877-622-5543 (toll free in the US) or +1-978-646-2777. 


\title{
Copyright permission (Figure 1-8)
}

\author{
Rightslink Printable License
}

\section{JOHN WILEY AND SONS LICENSE TERMS AND CONDITIONS}

This is a License Agreement between Megan L Killian ("You") and John Wiley and Sons ("John Wiley and Sons") provided by Copyright Clearance Center ("CCC"). The license consists of your order details, the terms and conditions provided by John Wiley and Sons, and the payment terms and conditions

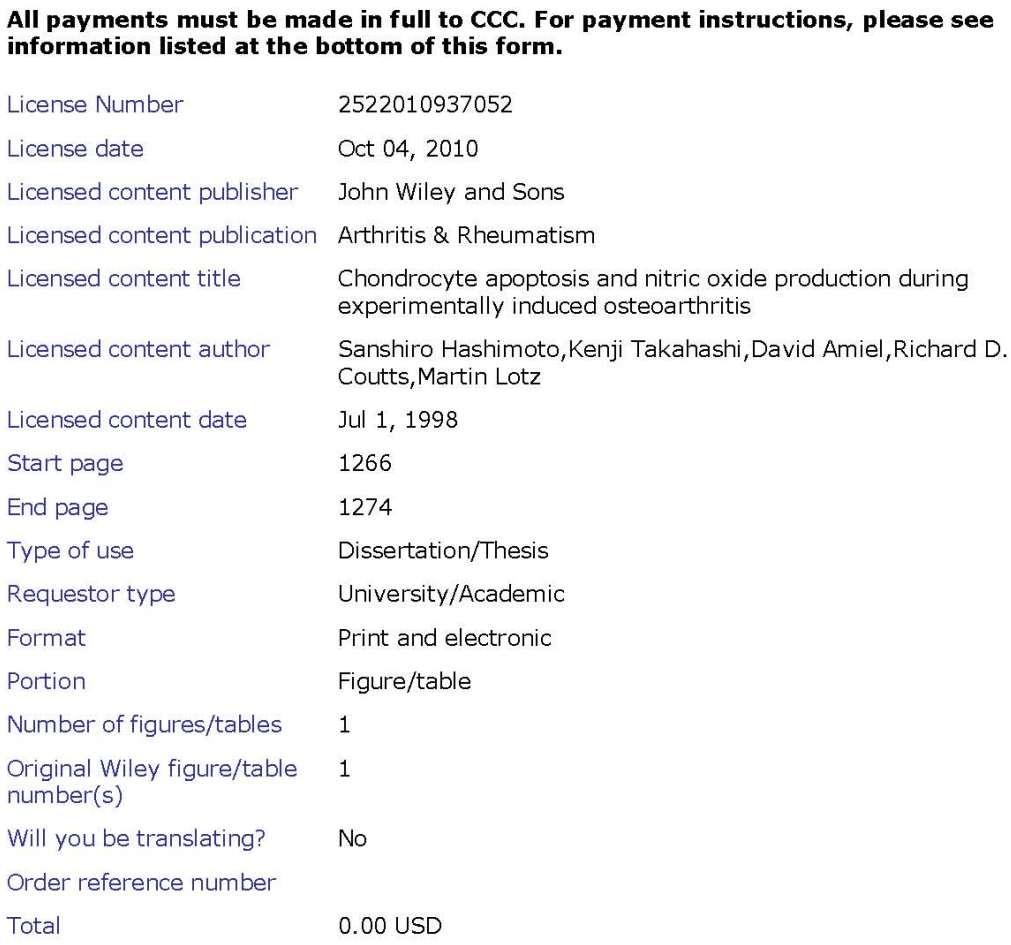

Terms and Conditions

\section{TERMS AND CONDITIONS}

This copyrighted material is owned by or exclusively licensed to John Wiley \& Sons, Inc. or one if its group companies (each a "Wiley Company") or a society for whom a Wiley Company has exclusive publishing rights in relation to a particular journal (collectively "WILEY"). By clicking "accept" in connection with completing this licensing transaction, you agree that the following terms and conditions apply to this transaction (along with the billing and payment terms and conditions established by the Copyright Clearance Center Inc., ("CCC's Billing and Payment terms and conditions"), at the time that you opened your Rightslink account (these are available at any time at http://myaccount.copyright.com).

Terms and Conditions

1. The materials you have requested permission to reproduce (the "Materials") are protected by 
copyright.

2. You are hereby granted a personal, non-exclusive, non-sublicensable, non-transferable, worldwide, limited license to reproduce the Materials for the purpose specified in the licensing process. This license is for a one-time use only with a maximum distribution equal to the number that you identified in the licensing process. Any form of republication granted by this licence must be completed within two years of the date of the grant of this licence (although copies prepared before may be distributed thereafter). Any electronic posting of the Materials is limited to one year from the date permission is granted and is on the condition that a link is placed to the journal homepage on Wiley's online journals publication platform at www. interscience. wiley.com. The Materials shall not be used in any other manner or for any other purpose. Permission is granted subject to an appropriate acknowledgement given to the author, title of the material/book/journal and the publisher and on the understanding that nowhere in the text is a previously published source acknowledged for all or part of this Material. Any third party material is expressly excluded from this permission.

3. With respect to the Materials, all rights are reserved. No part of the Materials may be copied, modified, adapted, translated, reproduced, transferred or distributed, in any form or by any means, and no derivative works may be made based on the Materials without the prior permission of the respective copyright owner. You may not alter, remove or suppress in any manner any copyright, trademark or other notices displayed by the Materials. You may not license, rent, sell, loan, lease, pledge, offer as security, transfer or assign the Materials, or any of the rights granted to you hereunder to any other person.

4. The Materials and all of the intellectual property rights therein shall at all times remain the exclusive property of John Wiley \& Sons Inc or one of its related companies (WILEY) or their respective licensors, and your interest therein is only that of having possession of and the right to reproduce the Materials pursuant to Section 2 herein during the continuance of this Agreement. You agree that you own no right, title or interest in or to the Materials or any of the intellectual property rights therein. You shall have no rights hereunder other than the license as provided for above in Section 2. No right, license or interest to any trademark, trade name, service mark or other branding ("Marks") of WILEY or its licensors is granted hereunder, and you agree that you shall not assert any such right, license or interest with respect thereto.

5. WILEY DOES NOT MAKE ANY WARRANTY OR REPRESENTATION OF ANY KIND TO YOU OR ANY THIRD PARTY, EXPRESS, IMPLIED OR STATUTORY, WITH RESPECT TO THE MATERIALS OR THE ACCURACY OF ANY INFORMATION CONTAINED IN THE MATERIALS, INCLUDING, WITHOUT LIMITATION, ANY IMPLIED WARRANTY OF MERCHANTABILITY, ACCURACY, SATISFACTORY QUALITY, FITNESS FOR A PARTICULAR PURPOSE, USABILITY, INTEGRATION OR NON-INFRINGEMENT AND ALL SUCH WARRANTIES ARE HEREBY EXCLUDED BY WILEY AND WAIVED BY YOU.

6. WILEY shall have the right to terminate this Agreement immediately upon breach of this Agreement by you.

7. You shall indemnify, defend and hold harmless WILEY, its directors, officers, agents and employees, from and against any actual or threatened claims, demands, causes of action or proceedings arising from any breach of this Agreement by you.

8. IN NO EVENT SHALL WILEY BE LIABLE TO YOU OR ANY OTHER PARTY OR ANY OTHER PERSON OR ENTITY FOR ANY SPECIAL, CONSEOUENTIAL, INCIDENTAL, INDIRECT, EXEMPLARY OR PUNITIVE DAMAGES, HOWEVER CAUSED, ARISING OUT OF OR IN CONNECTION WITH THE DOWNLOADING, PROVISIONING, VIEWING OR USE OF THE MATERIALS REGARDLESS OF THE FORM OF ACTION, WHETHER FOR BREACH OF CONTRACT, BREACH OF WARRANTY, TORT, NEGLIGENCE, INFRINGEMENT OR OTHERWISE (INCLUDING, WITHOUT LIMITATION, DAMAGES BASED ON LOSS OF PROFITS, DATA, FILES, USE, BUSINESS OPPORTUNITY OR CLAIMS OF THIRD PARTIES), AND WHETHER OR NOT THE PARTY HAS BEEN ADVISED OF THE POSSIBILITY OF SUCH DAMAGES. THIS LIMITATION SHALL APPLY NOTWITHSTANDING ANY FAILURE OF ESSENTIAL PURPOSE OF ANY LIMITED REMEDY PROVIDED HEREIN.

9. Should any provision of this Agreement be held by a court of competent jurisdiction to be illegal, invalid, or unenforceable, that provision shall be deemed amended to achieve as nearly as possible the same economic effect as the original provision, and the legality, validity and enforceability of the remaining provisions of this Agreement shall not be affected or impaired 
thereby.

10. The failure of either party to enforce any term or condition of this Agreement shall not constitute a waiver of either party's right to enforce each and every term and condition of this Agreement. No breach under this agreement shall be deemed waived or excused by either party unless such waiver or consent is in writing signed by the party granting such waiver or consent. The waiver by or consent of a party to a breach of any provision of this Agreement shall not operate or be construed as a waiver of or consent to any other or subsequent breach by such other party.

11. This Agreement may not be assigned (including by operation of law or otherwise) by you without WILEY's prior written consent.

12. These terms and conditions together with CCC's Billing and Payment terms and conditions (which are incorporated herein) form the entire agreement between you and WILEY concerning this licensing transaction and (in the absence of fraud) supersedes all prior agreements and representations of the parties, oral or written. This Agreement may not be amended except in a writing signed by both parties. This Agreement shall be binding upon and inure to the benefit of the parties' successors, legal representatives, and authorized assigns.

13. In the event of any conflict between your obligations established by these terms and conditions and those established by CCC's Billing and Payment terms and conditions, these terms and conditions shall prevail.

14. WILEY expressly reserves all rights not specifically granted in the combination of (i) the license details provided by you and accepted in the course of this licensing transaction, (ii) these terms and conditions and (iii) CCC's Billing and Payment terms and conditions.

15. This Agreement shall be governed by and construed in accordance with the laws of England and you agree to submit to the exclusive jurisdiction of the English courts.

16. Other Terms and Conditions:

BY CLICKING ON THE "I ACCEPT" BUTTON, YOU ACKNOWLEDGE THAT YOU HAVE READ AND FULLY UNDERSTAND EACH OF THE SECTIONS OF AND PROVISIONS SET FORTH IN THIS AGREEMENT AND THAT YOU ARE IN AGREEMENT WITH AND ARE WILLING TO ACCEPT ALL OF YOUR OBLIGATIONS AS SET FORTH IN THIS AGREEMENT.

V1.2

Gratis licenses (referencing $\$ 0$ in the Total field) are free. Please retain this printable license for your reference. No payment is required.

If you would like to pay for this license now, please remit this license along with your payment made payable to "COPYRIGHT CLEARANCE CENTER" otherwise you will be invoiced within $\mathbf{4 8}$ hours of the license date. Payment should be in the form of a check or money order referencing your account number and this invoice number

RLNK10860111.

Once you receive your invoice for this order, you may pay your invoice by credit card Please follow instructions provided at that time.

Make Payment To:

Copyright Clearance Center

Dept 001

P.O. Box 843006

Boston, MA 02284-3006

If you find copyrighted material related to this license will not be used and wish to cancel, please contact us referencing this license number 2522010937052 and noting the reason for cancellation.

Questions? customercare@copvriaht.com or +1-877-622-5543 (toll free in the US) or +1-978-646-2777. 


\section{Copyright permission (Figure 1-8)}

Rightslink Printable License

JOHN WILEY AND SONS LICENSE

TERMS AND CONDITIONS

Oct 19, 2010

This is a License Agreement between Megan L Killian ("You") and John Wiley and Sons ("John Wiley and Sons") provided by Copyright Clearance Center ("CCC"). The license consists of your order details, the terms and conditions provided by John Wiley and Sons, and the payment terms and conditions.

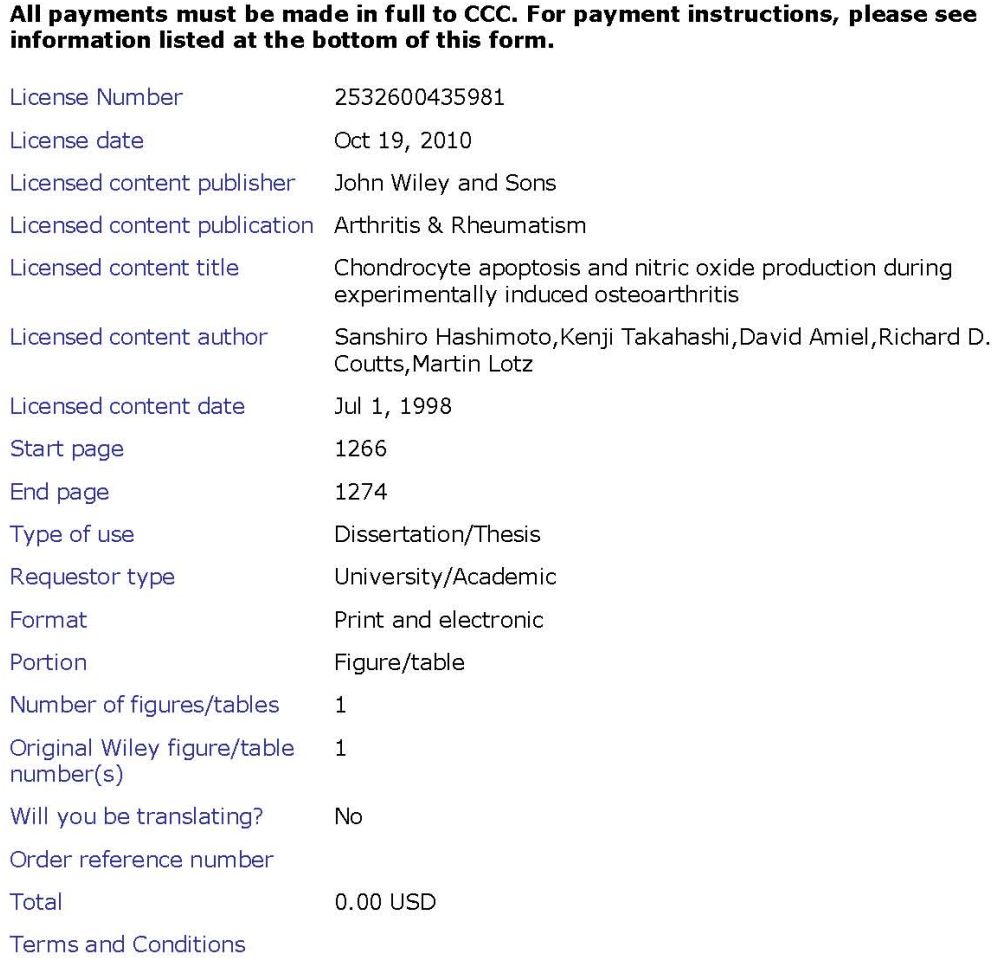

\section{TERMS AND CONDITIONS}

This copyrighted material is owned by or exclusively licensed to John Wiley \& Sons, Inc. or one if its group companies (each a "Wiley Company") or a society for whom a Wiley Company has exclusive publishing rights in relation to a particular journal (collectively "WILEY"). By clicking "accept" in connection with completing this licensing transaction, you agree that the following terms and conditions apply to this transaction (along with the billing and payment terms and conditions established by the Copyright Clearance Center Inc., ("CCC's Billing and Payment terms and conditions"), at the time that you opened your Rightslink account (these are available at any time at http://myaccount.copyright.com).

Terms and Conditions

1. The materials you have requested permission to reproduce (the "Materials") are protected by 
copyright.

2. You are hereby granted a personal, non-exclusive, non-sublicensable, non-transferable, worldwide, limited license to reproduce the Materials for the purpose specified in the licensing process. This license is for a one-time use only with a maximum distribution equal to the number that you identified in the licensing process. Any form of republication granted by this licence must be completed within two years of the date of the grant of this licence (although copies prepared before may be distributed thereafter). Any electronic posting of the Materials is limited to one year from the date permission is granted and is on the condition that a link is placed to the journal homepage on Wiley's online journals publication platform at www. interscience. wiley.com. The Materials shall not be used in any other manner or for any other purpose. Permission is granted subject to an appropriate acknowledgement given to the author, title of the material/book/journal and the publisher and on the understanding that nowhere in the text is a previously published source acknowledged for all or part of this Material. Any third party material is expressly excluded from this permission.

3. With respect to the Materials, all rights are reserved. No part of the Materials may be copied, modified, adapted, translated, reproduced, transferred or distributed, in any form or by any means, and no derivative works may be made based on the Materials without the prior permission of the respective copyright owner. You may not alter, remove or suppress in any manner any copyright, trademark or other notices displayed by the Materials. You may not license, rent, sell, loan, lease, pledge, offer as security, transfer or assign the Materials, or any of the rights granted to you hereunder to any other person.

4. The Materials and all of the intellectual property rights therein shall at all times remain the exclusive property of John Wiley \& Sons Inc or one of its related companies (WILEY) or their respective licensors, and your interest therein is only that of having possession of and the right to reproduce the Materials pursuant to Section 2 herein during the continuance of this Agreement. You agree that you own no right, title or interest in or to the Materials or any of the intellectual property rights therein. You shall have no rights hereunder other than the license as provided for above in Section 2. No right, license or interest to any trademark, trade name, service mark or other branding ("Marks") of WILEY or its licensors is granted hereunder, and you agree that you shall not assert any such right, license or interest with respect thereto.

5. WILEY DOES NOT MAKE ANY WARRANTY OR REPRESENTATION OF ANY KIND TO YOU OR ANY THIRD PARTY, EXPRESS, IMPLIED OR STATUTORY, WITH RESPECT TO THE MATERIALS OR THE ACCURACY OF ANY INFORMATION CONTAINED IN THE MATERIALS, INCLUDING, WITHOUT LIMITATION, ANY IMPLIED WARRANTY OF MERCHANTABILITY, ACCURACY, SATISFACTORY QUALITY, FITNESS FOR A PARTICULAR PURPOSE, USABILITY, INTEGRATION OR NON-INFRINGEMENT AND ALL SUCH WARRANTIES ARE HEREBY EXCLUDED BY WILEY AND WAIVED BY YOU.

6. WILEY shall have the right to terminate this Agreement immediately upon breach of this Agreement by you.

7. You shall indemnify, defend and hold harmless WILEY, its directors, officers, agents and employees, from and against any actual or threatened claims, demands, causes of action or proceedings arising from any breach of this Agreement by you.

8. IN NO EVENT SHALL WILEY BE LIABLE TO YOU OR ANY OTHER PARTY OR ANY OTHER PERSON OR ENTITY FOR ANY SPECIAL, CONSEOUENTIAL, INCIDENTAL, INDIRECT, EXEMPLARY OR PUNITIVE DAMAGES, HOWEVER CAUSED, ARISING OUT OF OR IN CONNECTION WITH THE DOWNLOADING, PROVISIONING, VIEWING OR USE OF THE MATERIALS REGARDLESS OF THE FORM OF ACTION, WHETHER FOR BREACH OF CONTRACT, BREACH OF WARRANTY, TORT, NEGLIGENCE, INFRINGEMENT OR OTHERWISE (INCLUDING, WITHOUT LIMITATION, DAMAGES BASED ON LOSS OF PROFITS, DATA, FILES, USE, BUSINESS OPPORTUNITY OR CLAIMS OF THIRD PARTIES), AND WHETHER OR NOT THE PARTY HAS BEEN ADVISED OF THE POSSIBILITY OF SUCH DAMAGES. THIS LIMITATION SHALL APPLY NOTWITHSTANDING ANY FAILURE OF ESSENTIAL PURPOSE OF ANY LIMITED REMEDY PROVIDED HEREIN.

9. Should any provision of this Agreement be held by a court of competent jurisdiction to be illegal, invalid, or unenforceable, that provision shall be deemed amended to achieve as nearly as possible the same economic effect as the original provision, and the legality, validity and enforceability of the remaining provisions of this Agreement shall not be affected or impaired 
thereby.

10. The failure of either party to enforce any term or condition of this Agreement shall not constitute a waiver of either party's right to enforce each and every term and condition of this Agreement. No breach under this agreement shall be deemed waived or excused by either party unless such waiver or consent is in writing signed by the party granting such waiver or consent. The waiver by or consent of a party to a breach of any provision of this Agreement shall not operate or be construed as a waiver of or consent to any other or subsequent breach by such other party.

11. This Agreement may not be assigned (including by operation of law or otherwise) by you without WILEY's prior written consent.

12. These terms and conditions together with CCC's Billing and Payment terms and conditions (which are incorporated herein) form the entire agreement between you and WILEY concerning this licensing transaction and (in the absence of fraud) supersedes all prior agreements and representations of the parties, oral or written. This Agreement may not be amended except in a writing signed by both parties. This Agreement shall be binding upon and inure to the benefit of the parties' successors, legal representatives, and authorized assigns.

13. In the event of any conflict between your obligations established by these terms and conditions and those established by CCC's Billing and Payment terms and conditions, these terms and conditions shall prevail.

14. WILEY expressly reserves all rights not specifically granted in the combination of (i) the license details provided by you and accepted in the course of this licensing transaction, (ii) these terms and conditions and (iii) CCC's Billing and Payment terms and conditions.

15. This Agreement shall be governed by and construed in accordance with the laws of England and you agree to submit to the exclusive jurisdiction of the English courts.

16. Other Terms and Conditions:

BY CLICKING ON THE "I ACCEPT" BUTTON, YOU ACKNOWLEDGE THAT YOU HAVE READ AND FULLY UNDERSTAND EACH OF THE SECTIONS OF AND PROVISIONS SET FORTH IN THIS AGREEMENT AND THAT YOU ARE IN AGREEMENT WITH AND ARE WILLING TO ACCEPT ALL OF YOUR OBLIGATIONS AS SET FORTH IN THIS AGREEMENT.

V1.2

Gratis licenses (referencing $\$ 0$ in the Total field) are free. Please retain this printable license for your reference. No payment is required.

If you would like to pay for this license now, please remit this license along with your payment made payable to "COPYRIGHT CLEARANCE CENTER" otherwise you will be invoiced within $\mathbf{4 8}$ hours of the license date. Payment should be in the form of a check or money order referencing your account number and this invoice number

RLNK10868812.

Once you receive your invoice for this order, you may pay your invoice by credit card. Please follow instructions provided at that time.

Make Payment To:

Copyright Clearance Center

Dept 001

P.o. Box 843006

Boston, MA 02284-3006

If you find copyrighted material related to this license will not be used and wish to cancel, please contact us referencing this license number 2532600435981 and noting the reason for cancellation.

Questions? customercare@copvriaht.com or $+1-877-622-5543$ (toll free in the US) or +1-978-646-2777 


\title{
Copyright permission (Chapter 2)
}

\author{
Rightslink Printable License
}

https://s100.copyright.com/CustomerAdmin/PrintableLicenseFrame.jsp?li...

This is a License Agreement between Megan L Killian ("You") and Elsevier ("Elsevier") provided by Copyright Clearance Center ("CCC"). The license consists of your order details, the terms and conditions provided by Elsevier, and the payment terms and conditions.

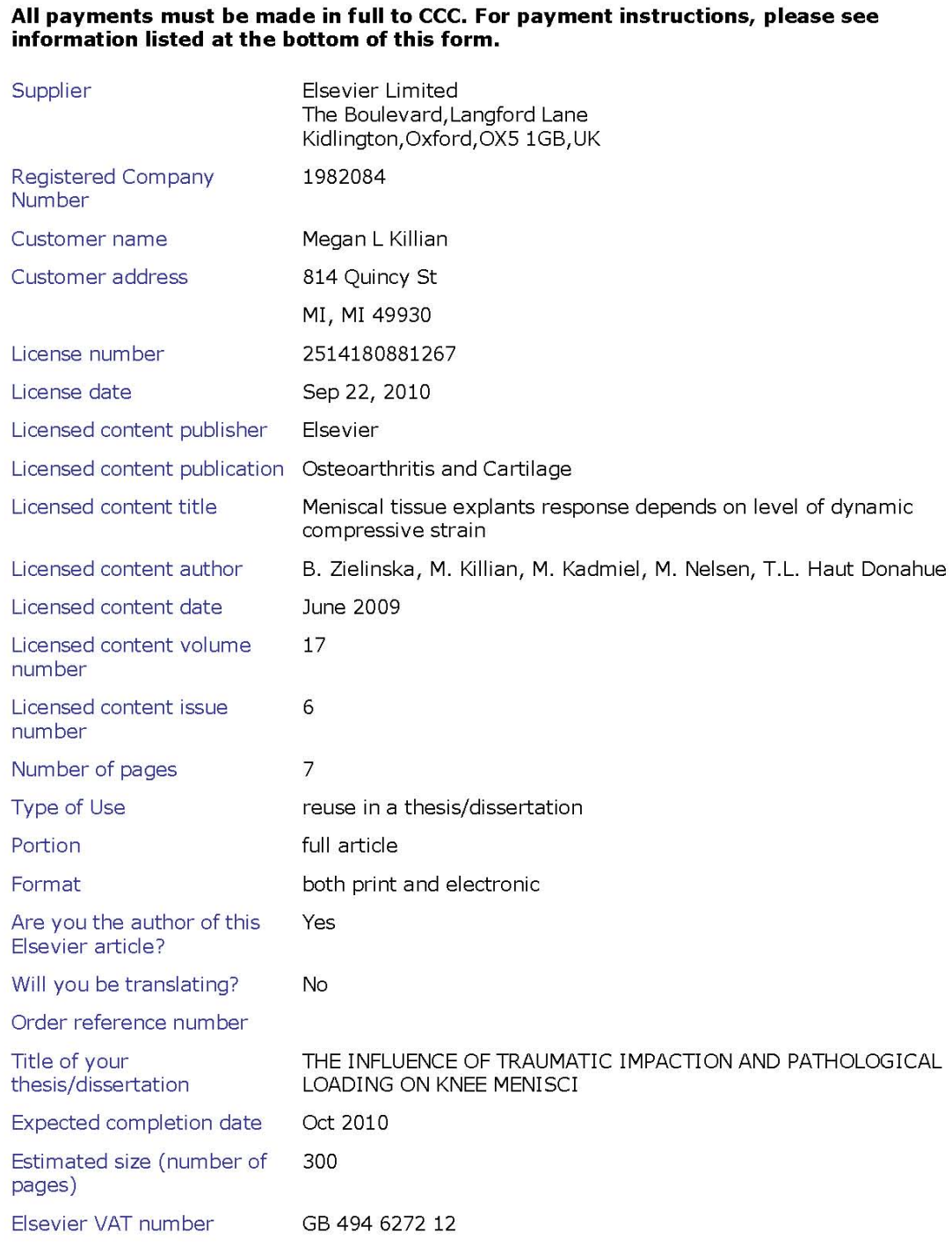

GB 494627212 
Terms and Conditions

\section{INTRODUCTION}

1. The publisher for this copyrighted material is Elsevier. By clicking "accept" in connection with completing this licensing transaction, you agree that the following terms and conditions apply to this transaction (along with the Billing and Payment terms and conditions established by Copyright Clearance Center, Inc. ("CCC"), at the time that you opened your Rightslink account and that are available at any time at http://myaccount.copyright.com).

\section{GENERAL TERMS}

2. Elsevier hereby grants you permission to reproduce the aforementioned material subject to the terms and conditions indicated.

3. Acknowledgement: If any part of the material to be used (for example, figures) has appeared in our publication with credit or acknowledgement to another source, permission must also be sought from that source. If such permission is not obtained then that material may not be included in your publication/copies. Suitable acknowledgement to the source must be made, either as a footnote or in a reference list at the end of your publication, as follows:

"Reprinted from Publication title, Vol/edition number, Author(s), Title of article / title of chapter, Pages No., Copyright (Year), with permission from Elsevier [OR APPLICABLE SOCIETY COPYRIGHT OWNER]." Also Lancet special credit - "Reprinted from The Lancet, Vol. number, Author(s), Title of article, Pages No., Copyright (Year), with permission from Elsevier."

4. Reproduction of this material is confined to the purpose and/or media for which permission is hereby given.

5. Altering/Modifying Material: Not Permitted. However figures and illustrations may be altered/adapted minimally to serve your work. Any other abbreviations, additions, deletions and/or any other alterations shall be made only with prior written authorization of Elsevier Ltd. (Please contact Elsevier at permissions@elsevier.com)

6. If the permission fee for the requested use of our material is waived in this instance, please be advised that your future requests for Elsevier materials may attract a fee.

7. Reservation of Rights: Publisher reserves all rights not specifically granted in the combination of (i) the license details provided by you and accepted in the course of this licensing transaction, (ii) these terms and conditions and (iii) CCC's Billing and Payment terms and conditions.

8. License Contingent Upon Payment: While you may exercise the rights licensed immediately upon issuance of the license at the end of the licensing process for the transaction, provided that you have disclosed complete and accurate details of your proposed use, no license is finally effective unless and until full payment is received from you (either by publisher or by $\mathrm{CCC}$ ) as provided in CCC's Billing and Payment terms and conditions. If full payment is not received on a timely basis, then any license preliminarily granted shall be deemed automatically revoked and shall be void as if never granted.

Further, in the event that you breach any of these terms and conditions or any of CCC's 
Billing and Payment terms and conditions, the license is automatically revoked and shall be void as if never granted. Use of materials as described in a revoked license, as well as any use of the materials beyond the scope of an unrevoked license, may constitute copyright infringement and publisher reserves the right to take any and all action to protect its copyright in the materials.

9. Warranties: Publisher makes no representations or warranties with respect to the licensed material.

10. Indemnity: You hereby indemnify and agree to hold harmless publisher and CCC, and their respective officers, directors, employees and agents, from and against any and all claims arising out of your use of the licensed material other than as specifically authorized pursuant to this license.

11. No Transfer of License: This license is personal to you and may not be sublicensed, assigned, or transferred by you to any other person without publisher's written permission.

12. No Amendment Except in Writing: This license may not be amended except in a writing signed by both parties (or, in the case of publisher, by CCC on publisher's behalf).

13. Objection to Contrary Terms: Publisher hereby objects to any terms contained in any purchase order, acknowledgment, check endorsement or other writing prepared by you, which terms are inconsistent with these terms and conditions or CCC's Billing and Payment terms and conditions. These terms and conditions, together with CCC's Billing and Payment terms and conditions (which are incorporated herein), comprise the entire agreement between you and publisher (and CCC) concerning this licensing transaction. In the event of any conflict between your obligations established by these terms and conditions and those established by CCC's Billing and Payment terms and conditions, these terms and conditions shall control.

14. Revocation: Elsevier or Copyright Clearance Center may deny the permissions described in this License at their sole discretion, for any reason or no reason, with a full refund payable to you. Notice of such denial will be made using the contact information provided by you. Failure to receive such notice will not alter or invalidate the denial. In no event will Elsevier or Copyright Clearance Center be responsible or liable for any costs, expenses or damage incurred by you as a result of a denial of your permission request, other than a refund of the amount(s) paid by you to Elsevier and/or Copyright Clearance Center for denied permissions.

\section{LIMITED LICENSE}

The following terms and conditions apply only to specific license types:

15. Translation: This permission is granted for non-exclusive world English rights only unless your license was granted for translation rights. If you licensed translation rights you may only translate this content into the languages you requested. A professional translator must perform all translations and reproduce the content word for word preserving the integrity of the article. If this license is to re-use 1 or 2 figures then permission is granted for non-exclusive world rights in all languages.

16. Website: The following terms and conditions apply to electronic reserve and author websites: 
Electronic reserve: If licensed material is to be posted to website, the web site is to be password-protected and made available only to bona fide students registered on a relevant course if:

This license was made in connection with a course,

This permission is granted for 1 year only. You may obtain a license for future website posting,

All content posted to the web site must maintain the copyright information line on the bottom of each image,

A hyper-text must be included to the Homepage of the journal from which you are licensing at http://www.sciencedirect.com/science/journal/xxxxx or the Elsevier homepage for books at http://www.elsevier.com, and

Central Storage: This license does not include permission for a scanned version of the material to be stored in a central repository such as that provided by Heron/XanEdu.

17. Author website for journals with the following additional clauses:

All content posted to the web site must maintain the copyright information line on the bottom of each image, and

he permission granted is limited to the personal version of your paper. You are not allowed to download and post the published electronic version of your article (whether PDF or HTML, proof or final version), nor may you scan the printed edition to create an electronic version,

A hyper-text must be included to the Homepage of the journal from which you are licensing at http:/www.sciencedirect.com/science/journal/xxxxx, As part of our normal production process, you will receive an e-mail notice when your article appears on Elsevier's online service ScienceDirect (www.sciencedirect.com). That e-mail will include the article's Digital Object Identifier (DOI). This number provides the electronic link to the published article and should be included in the posting of your personal version. We ask that you wait until you receive this e-mail and have the DOI to do any posting.

Central Storage: This license does not include permission for a scanned version of the material to be stored in a central repository such as that provided by Heron/XanEdu.

18. Author website for books with the following additional clauses: Authors are permitted to place a brief summary of their work online only. A hyper-text must be included to the Elsevier homepage at http://www.elsevier.com

All content posted to the web site must maintain the copyright information line on the bottom of each image

You are not allowed to download and post the published electronic version of your chapter, nor may you scan the printed edition to create an electronic version.

Central Storage: This license does not include permission for a scanned version of the material to be stored in a central repository such as that provided by Heron/XanEdu.

19. Website (regular and for author): A hyper-text must be included to the Homepage of the journal from which you are licensing at http://www.sciencedirect.com/science/journal /xxxxx. or for books to the Elsevier homepage at http://www.elsevier.com

20. Thesis/Dissertation: If your license is for use in a thesis/dissertation your thesis may be submitted to your institution in either print or electronic form. Should your thesis be 
published commercially, please reapply for permission. These requirements include permission for the Library and Archives of Canada to supply single copies, on demand, of the complete thesis and include permission for UMI to supply single copies, on demand, of the complete thesis. Should your thesis be published commercially, please reapply for permission.

\section{Other Conditions:}

v1.6

Gratis licenses (referencing $\$ 0$ in the Total field) are free. Please retain this printable license for your reference. No payment is required.

If you would like to pay for this license now, please remit this license along with your payment made payable to "COPYRIGHT CLEARANCE CENTER" otherwise you will be invoiced within $\mathbf{4 8}$ hours of the license date. Payment should be in the form of a check or money order referencing your account number and this invoice number

RLNK10853313.

Once you receive your invoice for this order, you may pay your invoice by credit card. Please follow instructions provided at that time.

Make Payment To:

Copyright Clearance Center

Dept 001

P.O. Box 843006

Boston, MA 02284-3006

If you find copyrighted material related to this license will not be used and wish to cancel, please contact us referencing this license number 2514180881267 and noting the reason for cancellation.

Questions? customercare@copvright.com or +1-877-622-5543 (toll free in the US) or +1-978-646-2777. 


\section{Copyright permission (Chapter 4)

\section{JOHN WILEY AND SONS LICENSE \\ TERMS AND CONDITIONS}

This is a License Agreement between Megan L Killian ("You") and John Wiley and Sons ("John Wiley and Sons") provided by Copyright Clearance Center ("CCC"). The license consists of your order details, the terms and conditions provided by John Wiley and Sons, and the payment terms and conditions.

\begin{tabular}{|c|c|}
\hline \multicolumn{2}{|c|}{$\begin{array}{l}\text { All payments must be made in full to CCC. For payment instructions, please see } \\
\text { information listed at the bottom of this form. }\end{array}$} \\
\hline License Number & 2552511163799 \\
\hline License date & Nov 19, 2010 \\
\hline Licensed content publisher & John Wiley and Sons \\
\hline Licensed content publication & $\begin{array}{l}\text { The Anatomical Record: Advances in Integrative Anatomy and } \\
\text { Evolutionary Biology }\end{array}$ \\
\hline Licensed content title & $\begin{array}{l}\text { Regional and Zonal Histo-Morphological Characteristics of the Lapine } \\
\text { Menisci }\end{array}$ \\
\hline Licensed content author & $\begin{array}{l}\text { Megan L. Killian, Nicole M. Lepinski, Roger C. Haut, Tammy L. Haut } \\
\text { Donahue }\end{array}$ \\
\hline Licensed content date & $\operatorname{Jan} 1,2010$ \\
\hline Start page & $\mathrm{n} / \mathrm{a}$ \\
\hline End page & $\mathrm{n} / \mathrm{a}$ \\
\hline Type of use & Dissertation/Thesis \\
\hline Requestor type & Author of this Wiley article \\
\hline Format & Print and electronic \\
\hline Portion & Full article \\
\hline Will you be translating? & No \\
\hline \multicolumn{2}{|l|}{ Order reference number } \\
\hline Total & 0.00 USD \\
\hline
\end{tabular}

\section{TERMS AND CONDITIONS}

This copyrighted material is owned by or exclusively licensed to John Wiley \& Sons, Inc. or one if its group companies (each a "Wiley Company") or a society for whom a Wiley Company has exclusive publishing rights in relation to a particular journal (collectively "WILEY"). By clicking "accept" in connection with completing this licensing transaction, you agree that the following terms and conditions apply to this transaction (along with the billing and payment terms and conditions established by the Copyright Clearance Center Inc., ("CCC's Billing and Payment terms and conditions"), at the time that you opened your Rightslink account (these are available at any time at http:/(myaccount. copyright.com).

Terms and Conditions

1. The materials you have requested permission to reproduce (the "Materials") are protected by copyright.

2. You are hereby granted a personal, non-exclusive, non-sublicensable, non-transferable, 
worldwide, limited license to reproduce the Materials for the purpose specified in the licensing process. This license is for a one-time use only with a maximum distribution equal to the number that you identified in the licensing process. Any form of republication granted by this licence must be completed within two years of the date of the grant of this licence (although copies prepared before may be distributed thereafter). Any electronic posting of the Materials is limited to one year from the date permission is granted and is on the condition that a link is placed to the journal homepage on Wiley's online journals publication platform at www. interscience. wiley.com. The Materials shall not be used in any other manner or for any other purpose. Permission is granted subject to an appropriate acknowledgement given to the author, title of the material/book/journal and the publisher and on the understanding that nowhere in the text is a previously published source acknowledged for all or part of this Material. Any third party material is expressly excluded from this permission.

3. With respect to the Materials, all rights are reserved. No part of the Materials may be copied, modified, adapted, translated, reproduced, transferred or distributed, in any form or by any means, and no derivative works may be made based on the Materials without the prior permission of the respective copyright owner. You may not alter, remove or suppress in any manner any copyright, trademark or other notices displayed by the Materials. You may not license, rent, sell, loan, lease, pledge, offer as security, transfer or assign the Materials, or any of the rights granted to you hereunder to any other person.

4. The Materials and all of the intellectual property rights therein shall at all times remain the exclusive property of John Wiley \& Sons Inc or one of its related companies (WILEY) or their respective licensors, and your interest therein is only that of having possession of and the right to reproduce the Materials pursuant to Section 2 herein during the continuance of this Agreement. You agree that you own no right, title or interest in or to the Materials or any of the intellectual property rights therein. You shall have no rights hereunder other than the license as provided for above in Section 2. No right, license or interest to any trademark, trade name, service mark or other branding ("Marks") of WILEY or its licensors is granted hereunder, and you agree that you shall not assert any such right, license or interest with respect thereto.

5. WILEY DOES NOT MAKE ANY WARRANTY OR REPRESENTATION OF ANY KIND TO YOU OR ANY THIRD PARTY, EXPRESS, IMPLIED OR STATUTORY, WITH RESPECT TO THE MATERIALS OR THE ACCURACY OF ANY INFORMATION CONTAINED IN THE MATERIALS, INCLUDING, WITHOUT LIMITATION, ANY IMPLIED WARRANTY OF MERCHANTABILITY, ACCURACY, SATISFACTORY QUALITY, FITNESS FOR A PARTICULAR PURPOSE, USABILITY, INTEGRATION OR NON-INFRINGEMENT AND ALL SUCH WARRANTIES ARE HEREBY EXCLUDED BY WILEY AND WAIVED BY YOU.

6. WILEY shall have the right to terminate this Agreement immediately upon breach of this Agreement by you.

7. You shall indemnify, defend and hold harmless WILEY, its directors, officers, agents and employees, from and against any actual or threatened claims, demands, causes of action or proceedings arising from any breach of this Agreement by you.

8. IN NO EVENT SHALL WILEY BE LIABLE TO YOU OR ANY OTHER PARTY OR ANY OTHER PERSON OR ENTITY FOR ANY SPECIAL, CONSEQUENTIAL, INCIDENTAL, INDIRECT, EXEMPLARY OR PUNITIVE DAMAGES, HOWEVER CAUSED, ARISING OUT OF OR IN CONNECTION WITH THE DOWNLOADING, PROVISIONING, VIEWING OR USE OF THE MATERIALS REGARDLESS OF THE FORM OF ACTION, WHETHER FOR BREACH OF CONTRACT, BREACH OF WARRANTY, TORT, NEGLIGENCE, INFRINGEMENT OR OTHERWISE (INCLUDING, WITHOUT LIMITATION, DAMAGES BASED ON LOSS OF PROFITS, DATA, FILES, USE, BUSINESS OPPORTUNITY OR CLAIMS OF THIRD PARTIES), AND WHETHER OR NOT THE PARTY HAS BEEN ADVISED OF THE POSSIBILITY OF SUCH DAMAGES. THIS LIMITATION SHALL APPLY NOTWITHSTANDING ANY FAILURE OF ESSENTIAL PURPOSE OF ANY LIMITED REMEDY PROVIDED HEREIN.

9. Should any provision of this Agreement be held by a court of competent jurisdiction to be illegal, invalid, or unenforceable, that provision shall be deemed amended to achieve as nearly as possible the same economic effect as the original provision, and the legality, validity and enforceability of the remaining provisions of this Agreement shall not be affected or impaired thereby.

10. The failure of either party to enforce any term or condition of this Agreement shall not 
constitute a waiver of either party's right to enforce each and every term and condition of this Agreement. No breach under this agreement shall be deemed waived or excused by either party unless such waiver or consent is in writing signed by the party granting such waiver or consent. The waiver by or consent of a party to a breach of any provision of this Agreement shall not operate or be construed as a waiver of or consent to any other or subsequent breach by such other party.

11. This Agreement may not be assigned (including by operation of law or otherwise) by you without WILEY's prior written consent.

12. These terms and conditions together with CCC's Billing and Payment terms and conditions (which are incorporated herein) form the entire agreement between you and WILEY concerning this licensing transaction and (in the absence of fraud) supersedes all prior agreements and representations of the parties, oral or written. This Agreement may not be amended except in a writing signed by both parties. This Agreement shall be binding upon and inure to the benefit of the parties' successors, legal representatives, and authorized assigns.

13. In the event of any conflict between your obligations established by these terms and conditions and those established by CCC's Billing and Payment terms and conditions, these terms and conditions shall prevail.

14. WILEY expressly reserves all rights not specifically granted in the combination of (i) the license details provided by you and accepted in the course of this licensing transaction, (ii) these terms and conditions and (iii) CCC's Billing and Payment terms and conditions.

15. This Agreement shall be governed by and construed in accordance with the laws of England and you agree to submit to the exclusive jurisdiction of the English courts.

16. Other Terms and Conditions:

BY CLICKING ON THE "I ACCEPT" BUTTON, YOU ACKNOWLEDGE THAT YOU HAVE READ AND FULLY UNDERSTAND EACH OF THE SECTIÓNS OF AND PROVISIONS SET FORTH IN THIS AGREEMENT AND THAT YOU ARE IN AGREEMENT WITH AND ARE WILLING TO ACCEPT ALL OF YOUR OBLIGATIONS AS SET FORTH IN THIS AGREEMENT.

V1.2

Gratis licenses (referencing $\$ 0$ in the Total field) are free. Please retain this printable license for your reference. No payment is required.

If you would like to pay for this license now, please remit this license along with your payment made payable to "COPYRIGHT CLEARANCE CENTER" otherwise you will be invoiced within $\mathbf{4 8}$ hours of the license date. Payment should be in the form of a check or money order referencing your account number and this invoice number RLNK10886858.

Once you receive your invoice for this order, you may pay your invoice by credit card. Please follow instructions provided at that time.

Make Payment To:

Copyright Clearance Center

Dept 001

P.O. Box 843006

Boston, MA 02284-3006

If you find copyrighted material related to this license will not be used and wish to cancel, please contact us referencing this license number 2552511163799 and noting the reason for cancellation.

Questions? customercare@copvriaht.com or +1-877-622-5543 (toll free in the US) or +1-978-646-2777. 


\title{
Copyright permission (Chapter 5)
}

\author{
Rightslink Printable License
}

This is a License Agreement between Megan L Killian ("You") and Elsevier ("Elsevier") provided by Copyright Clearance Center ("CCC"). The license consists of your order details, the terms and conditions provided by Elsevier, and the payment terms and conditions.

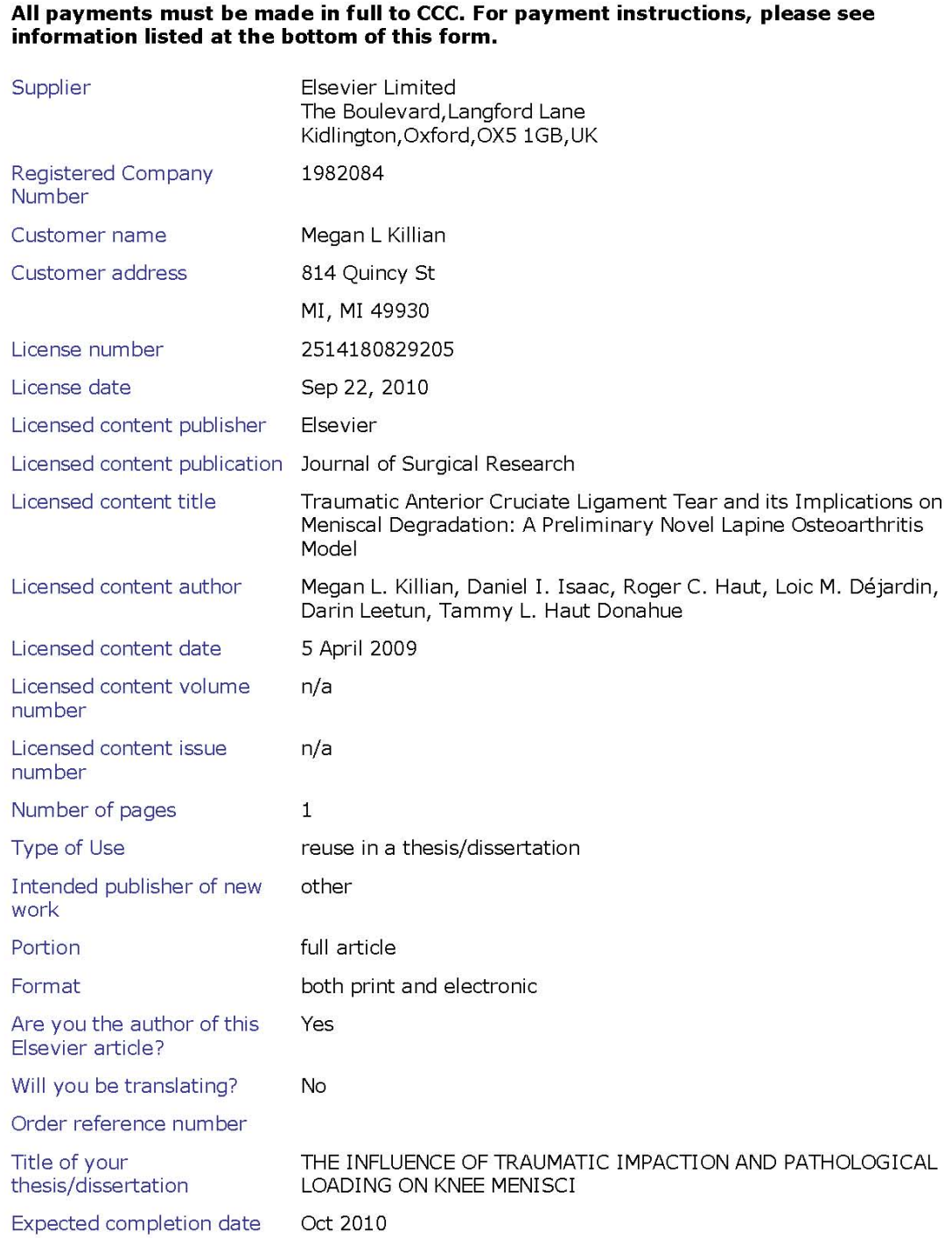




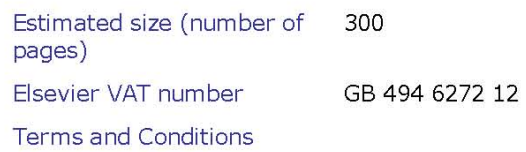

\section{INTRODUCTION}

1. The publisher for this copyrighted material is Elsevier. By clicking "accept" in connection with completing this licensing transaction, you agree that the following terms and conditions apply to this transaction (along with the Billing and Payment terms and conditions established by Copyright Clearance Center, Inc. ("CCC"), at the time that you opened your Rightslink account and that are available at any time at http://myaccount.copyright.com).

\section{GENERAL TERMS}

2. Elsevier hereby grants you permission to reproduce the aforementioned material subject to the terms and conditions indicated.

3. Acknowledgement: If any part of the material to be used (for example, figures) has appeared in our publication with credit or acknowledgement to another source, permission must also be sought from that source. If such permission is not obtained then that material may not be included in your publication/copies. Suitable acknowledgement to the source must be made, either as a footnote or in a reference list at the end of your publication, as follows:

"Reprinted from Publication title, Vol/edition number, Author(s), Title of article / title of chapter, Pages No., Copyright (Year), with permission from Elsevier [OR APPLICABLE SOCIETY COPYRIGHT OWNER]." Also Lancet special credit - "Reprinted from The Lancet, Vol. number, Author(s), Title of article, Pages No., Copyright (Year), with permission from Elsevier."

4. Reproduction of this material is confined to the purpose and/or media for which permission is hereby given.

5. Altering/Modifying Material: Not Permitted. However figures and illustrations may be altered/adapted minimally to serve your work. Any other abbreviations, additions, deletions and/or any other alterations shall be made only with prior written authorization of Elsevier Ltd. (Please contact Elsevier at permissions@elsevier.com)

6. If the permission fee for the requested use of our material is waived in this instance, please be advised that your future requests for Elsevier materials may attract a fee.

7. Reservation of Rights: Publisher reserves all rights not specifically granted in the combination of (i) the license details provided by you and accepted in the course of this licensing transaction, (ii) these terms and conditions and (iii) CCC's Billing and Payment terms and conditions.

8. License Contingent Upon Payment: While you may exercise the rights licensed immediately upon issuance of the license at the end of the licensing process for the transaction, provided that you have disclosed complete and accurate details of your proposed use, no license is finally effective unless and until full payment is received from you (either by publisher or by $\mathrm{CCC}$ ) as provided in CCC's Billing and Payment terms and 
conditions. If full payment is not received on a timely basis, then any license preliminarily granted shall be deemed automatically revoked and shall be void as if never granted. Further, in the event that you breach any of these terms and conditions or any of CCC's Billing and Payment terms and conditions, the license is automatically revoked and shall be void as if never granted. Use of materials as described in a revoked license, as well as any use of the materials beyond the scope of an unrevoked license, may constitute copyright infringement and publisher reserves the right to take any and all action to protect its copyright in the materials.

9. Warranties: Publisher makes no representations or warranties with respect to the licensed material.

10. Indemnity: You hereby indemnify and agree to hold harmless publisher and CCC, and their respective officers, directors, employees and agents, from and against any and all claims arising out of your use of the licensed material other than as specifically authorized pursuant to this license.

11. No Transfer of License: This license is personal to you and may not be sublicensed, assigned, or transferred by you to any other person without publisher's written permission.

12. No Amendment Except in Writing: This license may not be amended except in a writing signed by both parties (or, in the case of publisher, by CCC on publisher's behalf).

13. Objection to Contrary Terms: Publisher hereby objects to any terms contained in any purchase order, acknowledgment, check endorsement or other writing prepared by you, which terms are inconsistent with these terms and conditions or CCC's Billing and Payment terms and conditions. These terms and conditions, together with CCC's Billing and Payment terms and conditions (which are incorporated herein), comprise the entire agreement between you and publisher (and CCC) concerning this licensing transaction. In the event of any conflict between your obligations established by these terms and conditions and those established by CCC"s Billing and Payment terms and conditions, these terms and conditions shall control.

14. Revocation: Elsevier or Copyright Clearance Center may deny the permissions described in this License at their sole discretion, for any reason or no reason, with a full refund payable to you. Notice of such denial will be made using the contact information provided by you. Failure to receive such notice will not alter or invalidate the denial. In no event will Elsevier or Copyright Clearance Center be responsible or liable for any costs, expenses or damage incurred by you as a result of a denial of your permission request, other than a refund of the amount(s) paid by you to Elsevier and/or Copyright Clearance Center for denied permissions.

\section{LIMITED LICENSE}

The following terms and conditions apply only to specific license types:

15. Translation: This permission is granted for non-exclusive world English rights only unless your license was granted for translation rights. If you licensed translation rights you may only translate this content into the languages you requested. A professional translator must perform all translations and reproduce the content word for word preserving the integrity of the article. If this license is to re-use 1 or 2 figures then permission is granted for non-exclusive world rights in all languages. 
16. Website: The following terms and conditions apply to electronic reserve and author websites:

Electronic reserve: If licensed material is to be posted to website, the web site is to be password-protected and made available only to bona fide students registered on a relevant course if:

This license was made in connection with a course,

This permission is granted for 1 year only. You may obtain a license for future website posting,

All content posted to the web site must maintain the copyright information line on the bottom of each image,

A hyper-text must be included to the Homepage of the journal from which you are licensing at http://www.sciencedirect.com/science/journal/xxxxx or the Elsevier homepage for books at http://www.elsevier.com, and

Central Storage: This license does not include permission for a scanned version of the material to be stored in a central repository such as that provided by Heron/XanEdu.

17. Author website for journals with the following additional clauses:

All content posted to the web site must maintain the copyright information line on the bottom of each image, and

he permission granted is limited to the personal version of your paper. You are not allowed to download and post the published electronic version of your article (whether PDF or HTML, proof or final version), nor may you scan the printed edition to create an electronic version,

A hyper-text must be included to the Homepage of the journal from which you are licensing at http://www.sciencedirect.com/science/journal/xxxxx , As part of our normal production process, you will receive an e-mail notice when your article appears on Elsevier's online service ScienceDirect (www.sciencedirect.com). That e-mail will include the article's Digital Object Identifier (DOI). This number provides the electronic link to the published article and should be included in the posting of your personal version. We ask that you wait until you receive this e-mail and have the DOI to do any posting.

Central Storage: This license does not include permission for a scanned version of the material to be stored in a central repository such as that provided by Heron/XanEdu.

18. Author website for books with the following additional clauses: Authors are permitted to place a brief summary of their work online only.

A hyper-text must be included to the Elsevier homepage at http:/www.elsevier.com

All content posted to the web site must maintain the copyright information line on the bottom of each image

You are not allowed to download and post the published electronic version of your chapter, nor may you scan the printed edition to create an electronic version.

Central Storage: This license does not include permission for a scanned version of the material to be stored in a central repository such as that provided by Heron/XanEdu.

19. Website (regular and for author): A hyper-text must be included to the Homepage of the journal from which you are licensing at http://www.sciencedirect.com/science/journal

/xxxxx. or for books to the Elsevier homepage at http://www.elsevier.com 
20. Thesis/Dissertation: If your license is for use in a thesis/dissertation your thesis may be submitted to your institution in either print or electronic form. Should your thesis be published commercially, please reapply for permission. These requirements include permission for the Library and Archives of Canada to supply single copies, on demand, of the complete thesis and include permission for UMI to supply single copies, on demand, of the complete thesis. Should your thesis be published commercially, please reapply for permission.

\section{Other Conditions:}

v1.6

Gratis licenses (referencing $\$ 0$ in the Total field) are free. Please retain this printable license for your reference. No payment is required.

If you would like to pay for this license now, please remit this license along with your payment made payable to "COPYRIGHT CLEARANCE CENTER" otherwise you will be invoiced within $\mathbf{4 8}$ hours of the license date. Payment should be in the form of a check or money order referencing your account number and this invoice number RLNK10853311.

Once you receive your invoice for this order, you may pay your invoice by credit card. Please follow instructions provided at that time.

Make Payment To:

Copyright Clearance Center

Dept 001

P.O. Box 843006

Boston, MA 02284-3006

If you find copyrighted material related to this license will not be used and wish to cancel, please contact us referencing this license number 2514180829205 and noting the reason for cancellation.

Questions? customercare@copvright.com or +1-877-622-5543 (toll free in the US) or +1-978-646-2777. 


\section{IACUC}

\section{APPLICATION TO USE LIVING VERTEBRATE ANIMALS}

IN RESEARCH OR CLASSROOM SITUATIONS

The use of any animal in research and/or teaching without prior approval of the institutional animal care and use committee (IACUC) is a violation of Michigan Technological University policies and procedures. When completed, please send to the Research Integrity and Compliance Office, ATTN: Cheryl Gherna., 317A Administration Building. HANDWRITTEN APPLICATIONS WILL BE RETURNED LEAVE BLANK. \begin{tabular}{l|l} 
Date Recived & IACUC\# \\
\hline
\end{tabular} FOR OFFCE USE

\section{GENERAL INFORMATION}

Principal Investigator: Tammy Haut Donahue Department: MEEM E-mail: thdonahu@mtu.edu Co-Investigator ': Megan Killian Department: BME E-mail: mlkillia@mtu.edu

Title of Project: Immediate Treatment of Traumatic ACL Rupture: The Inflammatory Response of the Knee and Its Influence on Meniscal Tissue

Type of Application:

$\varangle$ New $\square$ Teaching Only $\square$ Information Only (non regulated) $\square$ Revision (within approval period) Previous Protocol \# L $\square$ Three-year Renewal Previous Protocol \# L

Project Duration: Start Date 4/1/2010 End Date 3/30/2012

Anticipated Funding Agency: NIH

\section{PROPOSED ANIMAL ACTIVITY}

Animal Housing Location: 315 M\&M Bldg.

Record Keeping: 927 MEEM-PI office

(State where the records involving the animals in the protocol will be kept and they can be made available for inspection by IACUC, USDA, or PHS staff members (if necessary)).

Animal Subjects - indicate species, code, and number of animals

\begin{tabular}{|l|l|l|l|l|l|l|l|}
\hline & \multicolumn{3}{|c|}{ Reporting Code* } & \multicolumn{2}{c|}{$\begin{array}{c}\text { Maximum } \\
\text { Daily }\end{array}$} & Total Number \\
\hline \multicolumn{1}{|c|}{ Species } & A & X & Y & Z & Population & Per Year \\
\hline Giant Flemish Rabbits & & & $\checkmark$ & & 14 & 60 \\
\hline & & & & & & \\
\hline
\end{tabular}

$\mathrm{A}^{*} \quad$ Animals which are not covered under the animal welfare act (these include laboratory mice/rats, birds, animals in agriculture research and cold blooded vertebrates)

$\mathrm{X}^{*} \quad$ Animals covered under the animal welfare act upon which teaching, research, experiments, or tests were conducted involving no pain, distress, or use of pain relieving drug.

Y* Animals covered under the animal welfare act upon which experiments, teaching, research, surgery, or tests were conducted involving accompanying pain or distress to the animals and for which appropriate anesthetic, analgesic, or tranquilizing drugs were used

$Z^{*} \quad$ Animals covered under the animal welfare act upon which teaching, experiments, research, surgery or tests were conducted involving accompanying pain or distress to the animals and for which the use of appropriate anesthetic, analgesic, or tranquilizing drugs would have adversely affected the procedures, results, or interpretation of the teaching, research, experiments, surgery, or tests. 
1. Animal Use Justification - explain why mathematical models, computer simulation, or in vitro biological systems cannot be used as acceptable alternatives to the use of animals in this project, describe the characteristics of the animal that justify its use in the proposed study, and describe how the number of animals needed for the study was determined.

See attached.

2. Description of the Proposed Activity - describe the proposed use of animals. The description should use non-technical language so that members of the IACUC that are not experts in your field can clearly understand the activities.

See attached

3. Assurance of Humane Treatment - describe how discomfort or injury to animals will be limited to what is unavoidable in scientifically valuable research. Indicate how you will minimize discomfort and pain by the use of appropriate analgesic, anesthetic, or tranquilizing drugs (description should include drugs, dosage, frequency of treatment and duration of treatment for each procedure).

See attached project description

4. Method of Euthanasia - provide a description of any euthanasia method to be used. If no euthanasia is anticipated but becomes necessary, describe method that would be used.

See attached project description 
5. Search for Alternatives to Painful Procedures - As per Animal Welfare Act regulations for procedures that MAY cause more than momentary or slight pain or distress to the animals, the Principal Investigator must provide a written narrative description of the methods used to determine that less painful/distressful alternatives are not available. A search for alternatives must be provided for EACH potentially painful/distressful procedure. Procedures that have pain eliminated by the use of anesthetics and/or analgesics are still considered painful even though the animal is not expected to experience any pain or distress. If alternative procedures are discovered the Principal Investigator must identify them and justify why those procedures are not being considered. Where specific testing procedures are required by Federal Law, the CFR references or other legal guidelines requiring them should also be noted.

\section{$\square$ N/A (Skip to 6)}

What strategy was used to determine that alternatives were not available:

Computer database search (eg Medline, Agricola, Biosis, etc): Database Name NLM-PubMed Years Covered 1990-2009

Database Name

Years Covered Date search performed May 28,2009 Keywords/Search Strategy

$\square$ Library Sources (journals, texts, reviews, etc)

$\square$ Consultations (who? when?)

$\square$ Other (please explain)

What were your findings with respect to alternatives to potentially painful procedures?

Findings were that there is no current non-animal alternative to study the potential development of a chronic joint disease from a single blunt impact. Concurrently, mathematical models will be developed from this data to model the development of the disease and possibly avoid future need for in vivo experiments.

6. Special Questions:

a) Are any animal restraint devices to be used in this project?

$\forall$ Yes

$\square$ No

if so, please describe:

see attached project description

b) Do activities require the use of any paralytic drugs?

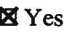

$\square$ No

if so, please describe:

tranquilizers; see attached project description

c) Do activities require any food or water deprivation?

$\square$ Yes

No

if so, please describe:

$\square$ Yes

区No

d) Do activities require any use of electrical shock?

if so, please describe:

e) Do activities result in any permanent physical impairment of the animals?

$\square$ Yes

No please describe:

f) Do activities unnecessarily duplicate previous experiments/activities?

$\square$ Yes

No describe and justify need for duplication:

$\square$ Yes

\No

Have animals identified for this project
activities/experiments? if so, explain:

h) Will animals involved in this project require any special care beyond the normal

$\square$ Yes

凶No 
7. Researcher Qualification - state the name(s) and describe qualifications of individual(s) conducting this study Tammy Haut Donahue, Associate Professor, MTU. Dr. Haut Donahue has a PhD in Biomedical Engineering and currently serves as the chair of the Institutional Animal Care and Use Committee.

Megan Killian, Graduate Student, Biomedical Engineering. Ms Killian is currently in research-only mode and has been trained for appropriate use of the animal facility and laboratory safety. 


\section{RATIONALE FOR ANIMAL USE}

1. Explain why mathematical models, computer simulations, or in vitro biological systems cannot be used as acceptable alternatives to the use of animals in this project. Describe the characteristics of the animal that justify its use in the proposed study, and describe how the number of animals needed for the study was determined.

The current study will use an in vivo animal model to study the temporal changes in the knee joint following blunt impact. This will provide evolution of damage to the articular cartilage and menisci following rupture of the ACL. We have previously conducted in vitro explant studies to provide the necessary preliminary data that will minimize the number of animals necessary in this current protocol. Both the cartilage and the menisci are not viable for long-term in vitro experiments, and thus chronic changes cannot be monitored over time. Since a primary object of the current study is to monitor the development of a post-traumatic degeneration and osteoarthritis in the tibio-femoral joint, and its potential limitation or mitigation with an acute intervention of joint lavage and/or corticosteroid, a live animal model is essential. To date, mathematical models cannot currently predict the chronic, or acute, responses of the body to blunt force impact trauma to a joint or predict its response to various posttrauma interventions. Additionally this animal model (the Flemish Giant rabbit) has been already established as part of a collaboration with Michigan State. It is hypothesized that impact-induced ligament and meniscal tearing will increase the production of $I L-1, M M P S$, and ADAMTS within the meniscus, leading to meniscal degeneration and compromised material properties. Furthermore, we hypothesize that joint lavage or a corticosteroid injection will remove or reduce IL-1 and other catabolic cytokines from the joint space, inhibiting trauma-induced meniscal matrix degeneration.

Rabbits are often used for orthopaedic studies. The animal is practical to maintain in reasonably large numbers for support of statistical interpretations, but it is also large enough for relevant mechanical and histological tissue sampling. The investigator has developed over the last year a collaboration with Michigan State using this animal model. We have visited the Michigan State site and learned from their practices. We are now branching to our own project and based on previous data collected in conjunction with Michigan State, it is apparent the Giant Flemish model will serve this purpose.

We have previous obtained meniscal tissue samples from Giant Flemish Rabbits that underwent a single blunt force impact to tear the ACL and menisci. Based on this data, published in the Journal of Surgical Research, a sample size of 10 animals in each group is necessary in order achieve statistical power to a temporal study. The sample size of 10 in each group is based on a power analysis to be able to detect a difference of 1 standard deviation in the mechanical properties of cartilage between impacted and unimpacted, contra-lateral limbs, with the known standard deviation to be approximately $30 \%$ of the mean. This level of difference has been shown to exist between clinically diagnosed normal and diseased tissue. We expect to see similar trends in meniscal tissue.

The following groups will be used. All animals will be allowed to acclimate for 2 weeks prior to intervention

Group 1: Control, time zero, no impact, no intervention. $n=10$

Group 2: 1 week, Impacted, no intervention. $n=10$

Group 3: 2 weeks, Impacted, no intervention. $n=10$

Group 4: 4 weeks, Impacted, no intervention. $n=10$

Group 5: 8 weeks, Impacted, no intervention. $n=10$

Group 6: 8 weeks, Impacted, Lavage @ day 3. n=10 
Group 7: 8 weeks, Impacted, Steriod @ day 3. n=10

Group 8: 8 weeks, Impacted, Placebo (saline) @day 3. n=10

Group 9: 8 weeks, Impacted, Lavage \& Steriod @ day3. n=10 


\section{PROJECT DESCRIPTION}

2. Describe the proposed use of animals. The description should use non-technical language so that members of the IACUC that are not experts in your field can clearly understand the activities.

This is a 2 year study. All animals will be procured through a single source (Doc's Rabbitry) and will be 6-8 month old Flemish Giant rabbits. Upon delivery, animals that appear too young, or sick, will be rejected at this point. Each animal will be quarantined upon arrival in a given room in the animal care facility, If necessary, Ivermectin $0.3 \mathrm{mg} / \mathrm{kg} \mathrm{S.Q.,} \mathrm{two} \mathrm{doses} 2$ weeks apart, will be given for control of internal and external parasites. Because these rabbits will not be specific pathogen free, we will treat all animals showing signs of pasturella with $10 \mathrm{mg} / \mathrm{kg}$ enrofloxacin injectable $10 \mathrm{mg} / \mathrm{kg} \mathrm{S.Q.} \mathrm{S.I.D.,} \mathrm{or} \mathrm{in}$ tablet form $10 \mathrm{mg} / \mathrm{kg}$ P.O. S.I.D. The animals will be closely monitored for approximately 2 weeks prior to being put into an experimental program. Each animal in Groups 2-5 indicated above, except those designated as controls, will be administered a blunt impact to the tibio-femoral joint at an intensity that will not induce bone fracture. We have previously collaborated with Michigan State using this procedure. At least 16 animals have previously received this treatment at our collaborators institute and those animals survived 1 year (12 months).

Prior to the blunt impact, each animal will be anesthetized with $0.75 \mathrm{mg} / \mathrm{kg}$ acepromazine S.Q., $5 \mathrm{mg} / \mathrm{kg}$ xylazine $(20 \mathrm{mg} / \mathrm{ml})$ I.M. and $35 \mathrm{mg} / \mathrm{kg}$ ketamine hydrochloride I.M. as a pre-anesthetic medication. The animals are typically under anesthesia for approximately 20 minutes.

While under anesthesia the right leg will be shaved to expose the proximal aspect of the knee. The area will be prepped with 3 scrubs, Betadine scrub and alcohol. The animal will be placed in a supine position with the right limb flexed approximately 90 degrees to align the tibia vertically (Figure 1). A shoe (similar to a ski boot) will be attached to the foot with leather straps that are secured with Velcro. Impacts will be delivered by dropping a mass onto the knee with a $4 \mathrm{~cm}$ square deformable interface (1.2 MPa Hexcel). This will insure that the impact loads are distributed over the femoral condyles preventing bone fracture. In previous studies a $1.33 \mathrm{~kg}$ mass was dropped from a height of $70 \mathrm{~cm}$ to load to the tibiofemoral joint without causing bone fractures (Figure 2).

The impacting mass will be arrested electronically to prevent multiple impacts. A load transducer (model 31/1432 Sensotec, Columbus, $\mathrm{OH}$ ) with a $500 \mathrm{lb}$ capacity will be attached behind the impact interface to record the load being applied to the knee. This can vary slightly between animals because of slightly changes in anatomy and restraint. Experimental data will be collected at $10 \mathrm{kHz}$ on a personal computer. Peak load and time to peak will be recorded by the graduate student.

Immediately after the experiment, the animals will be removed from the seat and given an injection of $0.02 \mathrm{mg} / \mathrm{kg}$ buprenorphine I.M. for pain. The animals will monitored for pain and treated, as needed, with additional doses of buprenorphine. Heart rate and respiratory rate are monitored during recovery. Animals will be allowed free cage activity without joint immobilization as this is similar to the clinical cases and in previous literature. Should we find pain to persist after the post-procedure, doses of buprenorphine will be administered and the rabbit examined. If pain cannot be mitigated with a safe dosage of buprenorphine, euthanasia will be considered. All rabbits will be treated with buprenorpine, .02-.05 mg/kg S.Q. every 12 hours for 72 hours, also consistent with the current literature. The first dose will be given prior to impact. The rabbits will be closely monitored for signs of pain (i.e. hunched appearance, lethargy, limping, decreased appetite, tooth grinding, and loss of interest in grooming)

All rabbits are euthanized at the end of each study. Acepromazine will be used as a tranquilizer prior to 
euthanasia, .25-3mg/kg S.Q. Acepromazine produces calmness and muscle relaxation, it also causes dilation of blood vessels which facilitates I.V. injection of fatal plus. Acepromazine is cost effective and not a controlled substance. The rabbits will be euthanized with Fatal Plus (pentobarbital) $85.9 \mathrm{mg} / \mathrm{kg} \mathrm{I.V}$.

Immediately after sacrifice of each animal the synovial fluid will be aspirated for biochemical analysis and viscosity measurements. The TF joint will be opened and grossly inspected and photographed for advanced pathology (loss of cartilage and exposure of bone). The structural integrity of the menisci will then be evaluated biomechanically and a complete biochemical analysis will ensue.

In the second year, Groups 6-9 will be tested. The objective is to show that lavage and/or corticosteroid administered immediately after injury will be effective at reducing joint damage. Previous studies on patients with Osteoarthritis already have not shown long-term effects with either lavage or corticosteroids. These experiments will have 10 test subjects for each group (6-9) for a total of 40 animals. In each of these studies the introduction of the blunt impacting force and the euthanasia of animals will follow that previously outlined above.

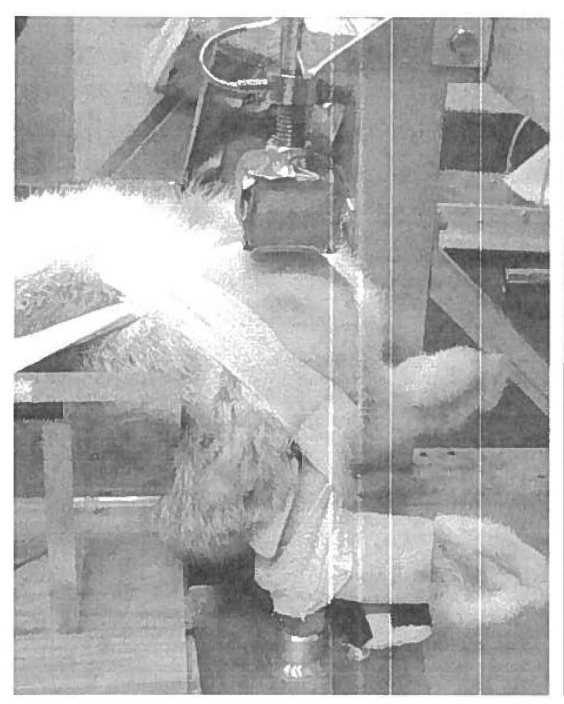

Fig 1 Rabbit knee flexed $90^{\circ}$ and secured with Velcro straps positioned beneath a deformable interface.

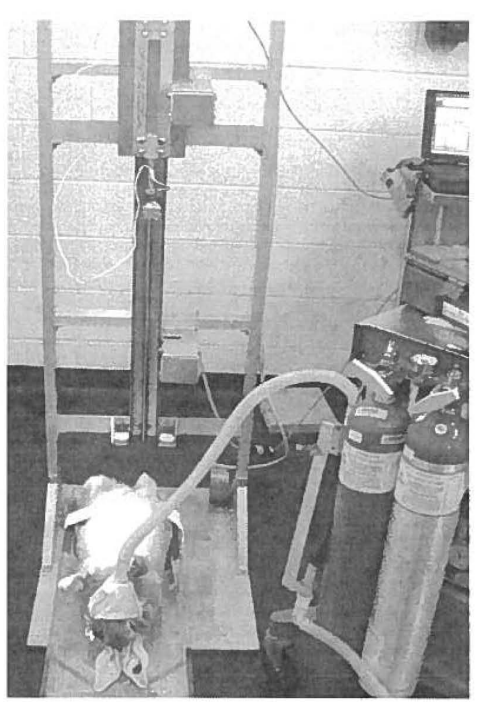

Fig 2 Anesthetised rabbit positioned for a gravityaccelerated mass impact. 
For Group 6: three days following trauma, animals will be anesthetized using previously described methods and both joints will be lavaged. Briefly, the injection site will be sterilely prepared using $10 \%$ povidone-iodine solution. Synovial fluid from the intraarticular joint space $(0.5 \mathrm{~mL})$ will be collected into sterile vials using a no-touch aseptic technique and 21-gauge sterile needles from both left and right knees. Following this, $50 \mathrm{ml}$ of sterile saline will be injected and removed into the left and right knee space with a syringe in $0.5 \mathrm{~mL}$ aliquots. The knee joints will then be manipulated through normal range of motion in order to disrupt fluid within the joints. The final injection of $0.5 \mathrm{~mL}$ saline will be retained within the joint. The wound will be cleaned and dressed upon removal of needles.

For Group 7\&8: Three days following trauma, animals will be anesthetized using previously described methods and both joints will receive intraarticular injections of methylprednisone (Group 7) or saline (Group 8).

For Group 9: Three days following trauma, animals will be anesthetized using previously described methods and both joints will be lavaged as in group 6, and then receive intraarticular injections of methylprednisone.

A permanent record of drug names, dosages, routes of administration and dates (times where appropriate) with all entries initialed will be maintained.

\section{ASSURANCE OF HUMANE TREATMENT}

3. Assurance of Humane Treatment - describe how discomfort or injury to animals will be limited to what is unavoidable in scientifically valuable research. Indicate how you will minimize discomfort and pain by the use of appropriate analgesic, anesthetic, or tranquilizing drugs (description should include drugs, dosage, frequency of treatment and duration of treatment for each procedure).

Detailed are provided in the project description above. Summarized here:

1. Ivermectin $0.3 \mathrm{mg} / \mathrm{kg} \mathrm{S.Q.} \mathrm{Two} \mathrm{doses} 2$ weeks apart will be given for control of internal and external parasites. Because these rabbits will not be specific pathogen free, we will treat all animals showing signs of pasturella with $10 \mathrm{mg} / \mathrm{kg}$ enrofloxacin injectable $10 \mathrm{mg} / \mathrm{kg}$ S.Q. S.I.D., or in tablet form $10 \mathrm{mg} / \mathrm{kg}$ P.O. S.I.D.

2. anesthetized with $0.75 \mathrm{mg} / \mathrm{kg}$ acepromazine S.Q., $5 \mathrm{mg} / \mathrm{kg}$ xylazine $(20 \mathrm{mg} / \mathrm{ml}) \mathrm{I} . \mathrm{M}$. and 35 $\mathrm{mg} / \mathrm{kg}$ ketamine hydrochloride I.M.

3. rabbits will be treated with buprenorpine, $.02-.05 \mathrm{mg} / \mathrm{kg} \mathrm{S.Q.} \mathrm{every} 12$ hours for 72 hours, also consistent with the current literature.

4. Acepromazine will be used as a tranquilizer prior to euthanasia, .25-3mg/kg S.Q.

5. The rabbits will be euthanized with Fatal Plus (pentobarbital) $85.9 \mathrm{mg} / \mathrm{kg} \mathrm{I.V}$.

\section{METHOD OF EUTHANASIA}

4. provide a deseription of any euthanasia method to be used. If no euthanasia is anticipated but becomes necessary, describe method that would be used.

\begin{tabular}{|c|c|c|c|}
\hline Species & Generic name only of agent & Dose $(\mathrm{mg} / \mathrm{kg})$ & Anatomical site \& route of administration \\
\hline rabbit & $\begin{array}{c}\text { Pentobarbital (fatal } \\
\text { plus) }\end{array}$ & 85.9 & IV
\end{tabular}




\section{MEMO}

TO: Dr. Tammy Haut Donahue, MEEM

\section{CC: Megan Killian, BME}

FROM: Joanne Polzien, Director Research Integrity and Compliance

DATE: March 11, 2010

SUBJECT: Continue Approval L0191

Protocol \#L0191

Project Title: "Influence of Traumatic Impaction of Cell Viability and Biochemistry in the Rabbit Knee"

This continued approval is valid for the period January 20, 2010 through January 19, 2011.

Your animal application continuation was disseminated and reviewed by the Institutional Animal Care and Use Committee (IACUC). The Committee has approved continuation of the application, with change in euthanasia method, and it is now on file in the Research Compliance Office. Approvals are granted for up to a one year period. You will need to request a continuation for each year of the project six weeks prior to the end date indicated above. The Research Integrity and Compliance Office will make every effort to send the Principal Investigator annual reminders. However, the Principal Investigator is responsible for submitting annual Continuation Forms in advance of the expiration date for the project. It is very important that these expiration dates are not missed. Failure to submit annual review materials on time will result in the termination of this protocol.

If you have any questions, please contact me at 487-2902 or jpolzien@mtu.edu. 